\title{
Degradation of fuel components
}

(4)

a1i.

initis.
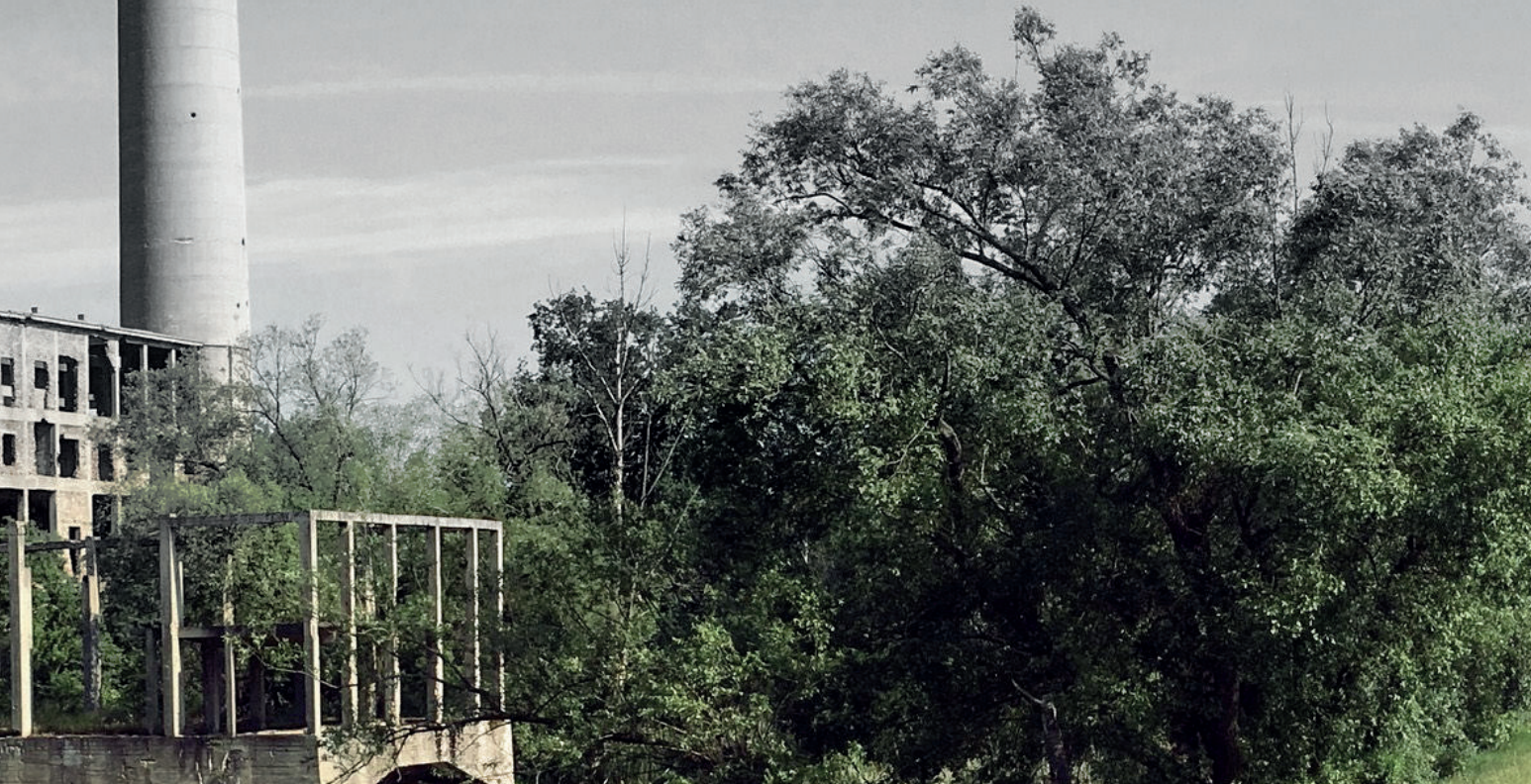

and

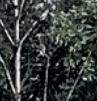

-

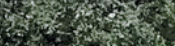

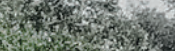
1.7. 3.1. 1

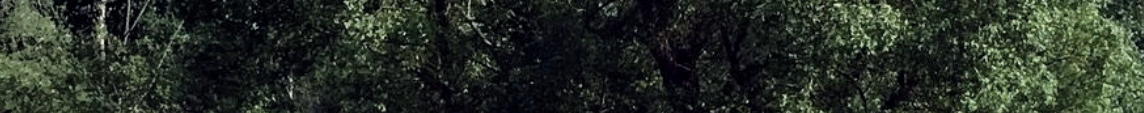
${ }_{2}$

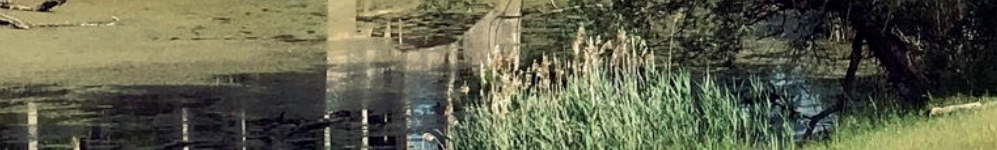

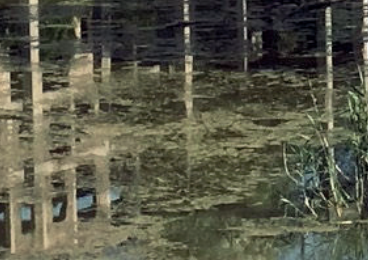
Marcelle J. van der Waals 



\section{Degradation of fuel components}

Johanna Marcelle van der Waals 


\section{Thesis committee}

\section{Promotor}

Prof. Dr H. Smidt

Personal chair at the Laboratory of Microbiology

Wageningen University \& Research

\section{Co-promotor}

Dr J. Gerritse

Senior Researcher

Deltares, Utrecht, the Netherlands

\section{Other members}

Prof. Dr T.W. Kuyper, Wageningen University \& Research

Dr J.R. Parsons, University of Amsterdam

Prof. Dr C. Vogt, Helmholtz-Centre for Environmental Research, Leipzig, Germany

Prof. Dr M. Häggblom, Rutgers, The State University of New Jersey, New Brunswick, USA

This research was conducted under the auspices of the Netherlands Graduate School for Socio-Economic and Natural Sciences of the Environment. 


\title{
Degradation of fuel components
}

\author{
Marcelle J. van der Waals
}

\section{Thesis}

submitted in fulfilment of the requirements for the degree of doctor at Wageningen University

by the authority of the Rector Magnificus,

Prof. Dr A.P.J. Mol,

in the presence of the

Thesis Committee appointed by the Academic Board

to be defended in public

on Thursday 18 October 2018

at 11 a.m. in the Aula. 
Marcelle J. van der Waals

Degradation of fuel components,

175 pages.

PhD thesis, Wageningen University, Wageningen, the Netherlands (2018)

With references, with summaries in English and Dutch

ISBN: 978-94-6343-315-0

DOI: https://doi.org/10.18174/454763 


\section{Table of contents}

Chapter 1. General introduction and thesis outline ........................................................ 7

Chapter 2. Benzene degradation in a denitrifying biofilm reactor: activity and microbial

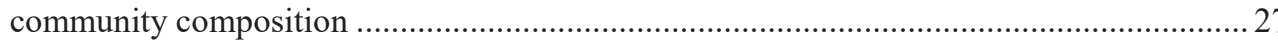

Chapter 3. Anaerobic degradation of a mixture of MtBE, EtBE, TBA and benzene under

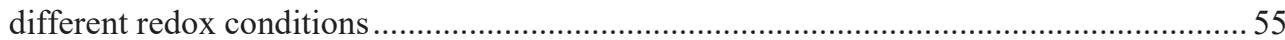

Chapter 4. Ethyl tert-butyl ether (EtBE) degradation by an algal-bacterial culture ............ 83

Chapter 5. Isolation of anaerobic benzene degrading bacteria and assessment of

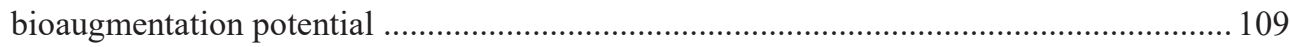

Chapter 6. General discussion and perspectives ........................................................... 131

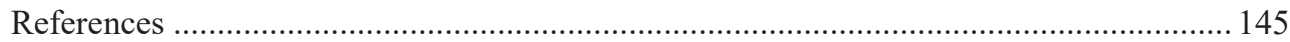

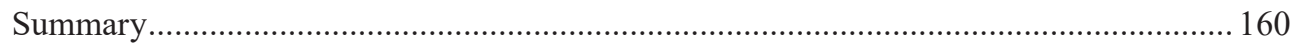

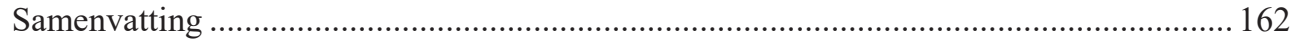

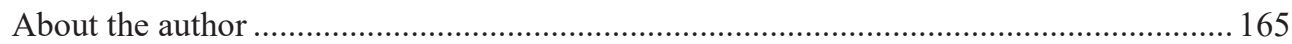

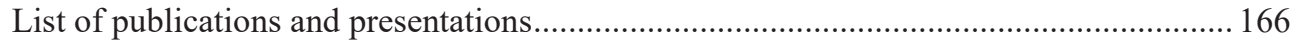

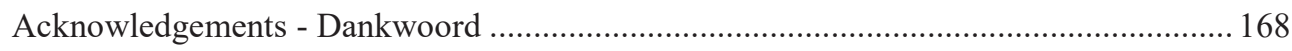

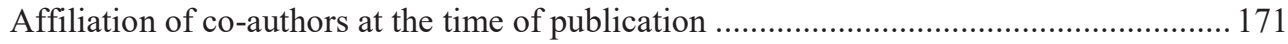


Chapter 1. General introduction and thesis outline 


\section{Chapter 1.}

\section{General introduction}

The subsurface with its respective groundwater is highly important for life on Earth. Groundwater is extracted at an estimated global rate of $982 \mathrm{~km}^{3} /$ year of which $70 \%$ is used for agriculture [1]. Other purposes include domestic and industrial usage. Almost half of our drinking water worldwide is supplied from groundwater [2]. Changes in groundwater quality may impact human health, livelihoods, food security and the economic development [2]. As many ecosystems depend on groundwater, it is important to take care of our environment and ensure the quality of groundwater for its many purposes.

\section{Subsurface contamination}

In the environment, contamination of soil and groundwater often occurs due to anthropogenic activity. Accidental spills, leaking storage tanks or inappropriate waste disposal have affected the surrounding environment (Figure 1.1) [3].

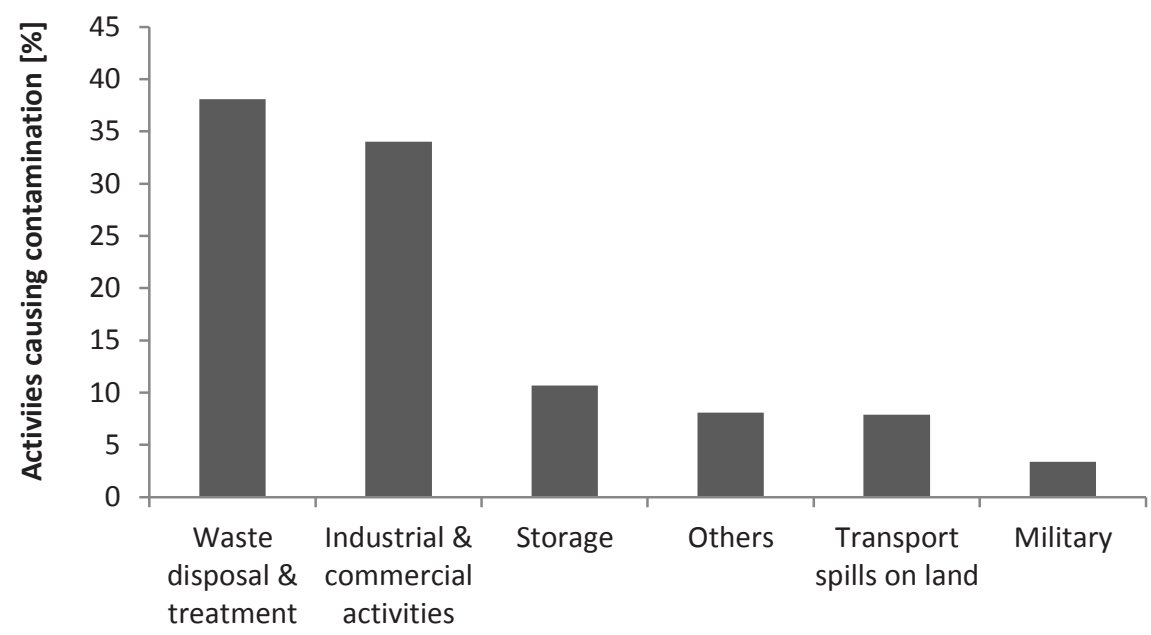

Figure 1.1 Activities causing contamination of the subsurface in Europe as a percentage of sources over the total number of sources identified. Nuclear operations contaminate in $0.1 \%$ of the total number of sources the subsurface in Europe. Figure adapted from the European Environment Agency (EEA) [3].

In the Netherlands, around 300.000 potentially polluted sites were identified in 2009 [4]. Between 4800 and 7000 locations required urgent remediation of which 1200 - 1800 locations posed human risks. The types of chemicals in the subsurface of the Netherlands are quite diverse, including aromatic hydrocarbons and mineral oil (Figure 1.2). 

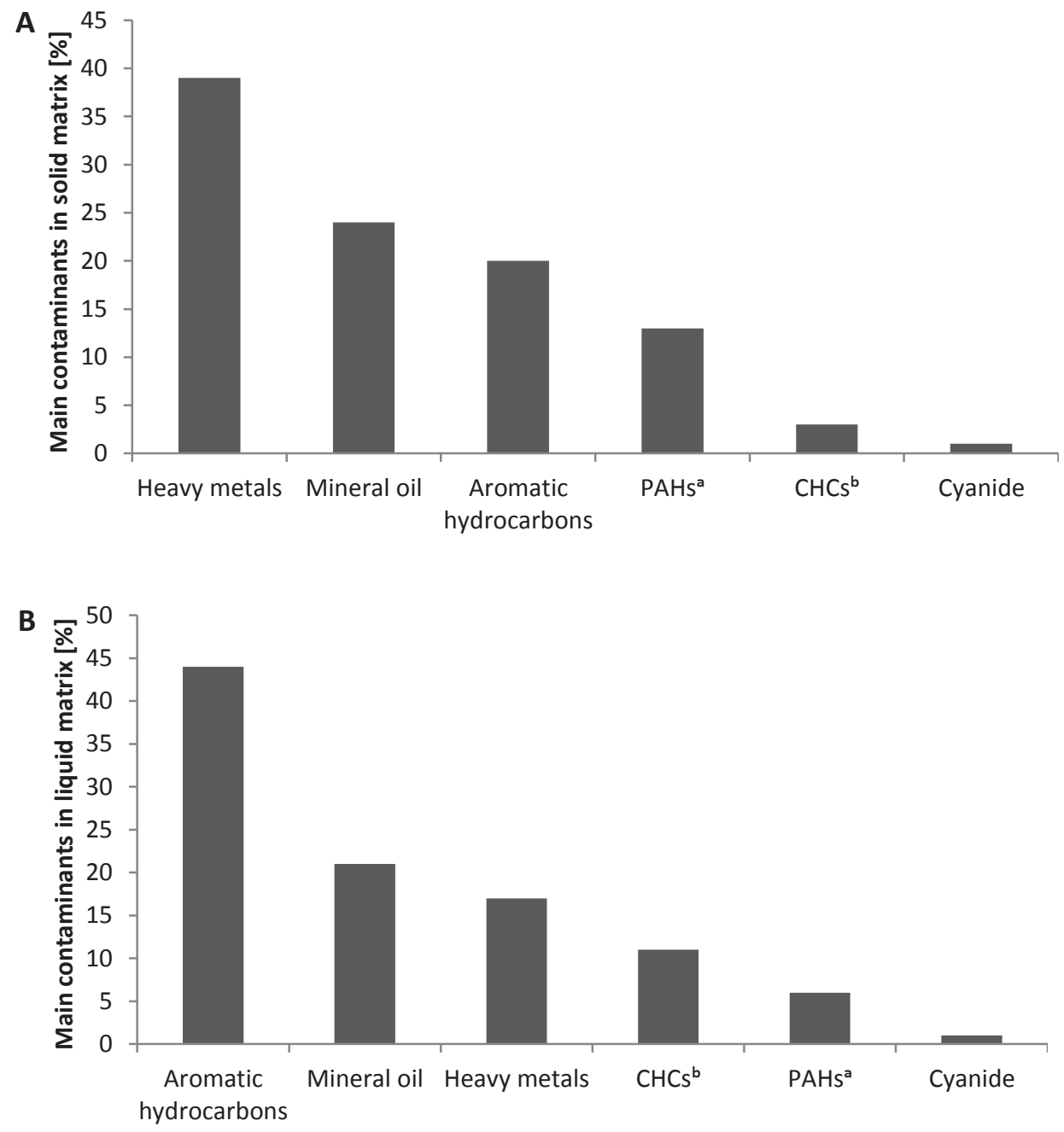

Figure 1.2 The main contaminants in the Netherlands in solid (A) and liquid (B) matrices. Figures adapted based on the EEA [5].

${ }^{\text {a }}$ PAHs: polycyclic aromatic hydrocarbons

${ }^{\mathrm{b}}$ CHCs: chlorinated hydrocarbons

The use of renewable resources, such as biomass from animal fat, soy oil and rapeseed oil as basis for automotive fuels is stimulated under the European Biofuels Directive (EC 2003) to reduce greenhouse gas emissions and to accelerate the transition to a biobased economy [6]. All EU member states have to add $10 \% \mathrm{v} / \mathrm{v}$ renewable fuel components by 2020 , for all 


\section{Chapter 1.}

transport options. In the Netherlands, the share of renewable energy in the transport sector has increased from $4.5 \%$ to $5.7 \%$ from 2012 to 2014 [7]. As biofuels becoming more widely used, their environmental fate is becoming an area of concern. Like in fossil fuels, also in biofuels additives are used [8]. Methyl tert-butyl ether (MtBE) is frequently used as a fuel additive to increase the fuel compressibility as characterised by the octane number and to decrease the emission of pollutants during combustion. In the past years, MtBE has been partially replaced by ethyl tert-butyl ether (EtBE), a fuel additive comparable with MtBE but partially produced from biobased resources such as bioethanol. Hence, it can be anticipated that, based on the rapidly increased use of renewable resources, current contaminants as well as those derived from renewable biomass will increasingly co-occur in contaminated environments.

\section{Fossil- and biobased fuels and associated contaminants}

Automotive fuels are produced from both fossil- and biobased resources [9]. The research described in this thesis will focus on the biodegradation of both fossil- and biobased derived organic contaminants. Selected contaminants include MtBE, EtBE, tert-butyl alcohol (TBA) and benzene of which MtBE, EtBE and TBA are gasoline oxygenates [10], whereas benzene is a constituent of crude oil and gasoline. These contaminants were selected in order to include three fossil-based contaminants (MtBE and benzene) and a biobased contaminant (EtBE). TBA is being produced as metabolite during MtBE and EtBE degradation, but is also frequently used as a polar organic solvent in flavours, perfumes or paint removers. Benzene is often considered as risk determining compound at fuel-contaminated locations, because it easily dissolves and transports in groundwater and is toxic [11]. MtBE, EtBE and TBA were selected because of the widespread problem due to their high water solubility, low odour and taste values and health concerns that require remediation [12-14].

In 2010, fifty-five fuel oxygenate production facilities with MtBE, EtBE and tertamyl methyl ether (TAME) were located in the European Union [1]. The total production capacity for MtBE, EtBE and TAME has grown from 4 kilotons in 2002 to 6 kilotons per year in 2010. The relative use of EtBE increased from 15\% in 2002 to $60 \%$ in 2010 while the MtBE production capacity decreased. There is no data available with respect to TBA production for blending with gasoline in Europe. Gasoline ether oxygenates (GEOs) are blended with gasoline in different concentrations depending on the gasoline type and coun- 
try legislation. The total annual gasoline demand in EU27, Croatia, Norway and Switzerland in 2007 was 104,340 kilotons. The production of benzene in 2016 was 7 kilotons in EU15 and Norway [15].

Table 1.1 describes the physico-chemical properties of the fossil- and biobased compounds selected for the research described in this thesis. Besides determining the biodegradation potential for these contaminants individually, we also evaluated the anaerobic degradation of contaminants in mixtures of fossil- and biobased derived sources. 
Chapter 1.

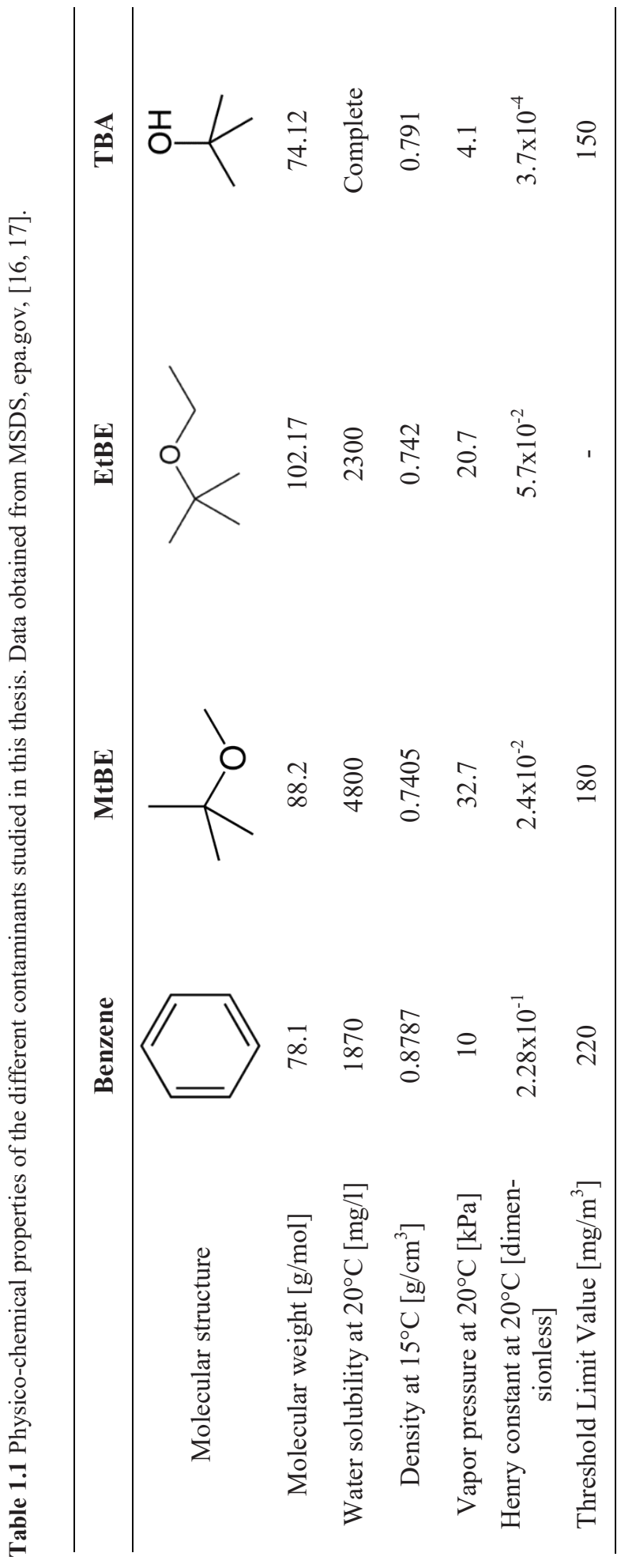




\section{Anaerobic degradation}

Oxygen is often rapidly depleted in contaminated groundwater by aerobic microorganisms using easily degradable substrates [18]. Consequently, anaerobic biodegradation is essential for contaminant removal in polluted subsurface environments. In the research described in this thesis we focused on degradation of selected contaminants without external oxygen supply.

\section{Anaerobic degradation of the fuel oxygenates MtBE and EtBE}

MtBE has been shown to be anaerobically degraded to the end product $\mathrm{CO}_{2}$ and/or metabolites (e.g. TBA) under a variety of redox conditions, including nitrate-, manganese(IV)-, iron(III)-, and sulphate-reducing and methanogenic conditions [19] (Table 1.2).

In contrast, little is known to date regarding anaerobic EtBE degradation. Previous studies reported in literature have shown lack of anaerobic degradation of EtBE [20]. However, Yeh and Novak (1994) found anaerobic EtBE degradation in soils under denitrifying and methanogenic conditions, and Bombach et al. (2015) suggested biodegradation of EtBE along an anoxic plume.

Table 1.2 Reported MtBE and EtBE degradation rates, with rates of $0.02 \mu \mathrm{M} /$ day and higher, under anoxic conditions using sulphate, iron(III), anthraquinone-2,6-disulfonate (AQDS), manganese(IV) or fumurate as electron acceptors.

\begin{tabular}{|c|c|c|c|}
\hline $\begin{array}{c}\text { Type of exper- } \\
\text { iment }\end{array}$ & Condition & $\begin{array}{c}\text { Degradation rate } \\
{[\mu \mathrm{M} / \mathrm{day}]}\end{array}$ & References \\
\hline \multicolumn{4}{|l|}{$\underline{\text { MtBE }}$} \\
\hline $\begin{array}{l}\text { Enrichment } \\
\text { culture }\end{array}$ & Methanogenic & 47 & {$[23]$} \\
\hline $\begin{array}{l}\text { Enrichment } \\
\text { culture }\end{array}$ & Methanogenic & 5.9 & {$[24]$} \\
\hline $\begin{array}{l}\text { Enrichment } \\
\text { culture }\end{array}$ & Methanogenic & $1.1-1.7$ & {$[25]$} \\
\hline $\begin{array}{l}\text { Enrichment } \\
\text { culture }\end{array}$ & Iron(III)-reducing & $2.8-5.6$ & {$[26]$} \\
\hline \multirow[t]{3}{*}{$\begin{array}{l}\text { Enrichment } \\
\text { culture }\end{array}$} & $\mathrm{AQDS}^{\mathrm{a}}$ addition & $35.6 \pm 3.8$ & {$[27]$} \\
\hline & Sulphate-reducing & $61.7 \pm 27.5$ & \\
\hline & Fumarate addition & $80.6 \pm 14.1$ & \\
\hline
\end{tabular}


Chapter 1.

\begin{tabular}{|c|c|c|c|}
\hline $\begin{array}{l}\text { Enrichment } \\
\text { culture }\end{array}$ & Nitrate-reducing & 0.45 & {$[28]$} \\
\hline $\begin{array}{l}\text { Enrichment } \\
\text { culture }\end{array}$ & Iron(III)-reducing & 0.88 & [29] \\
\hline \multirow[t]{5}{*}{$\begin{array}{l}\text { Enrichment } \\
\text { culture }\end{array}$} & Nitrate-reducing & $0.02-0.08$ & {$[30,31]$} \\
\hline & $\begin{array}{l}\text { Manganese(IV)- } \\
\text { reducing }\end{array}$ & 0.02 & \\
\hline & Iron(III)-reducing & 0.03 & \\
\hline & Sulphate-reducing & 0.03 & \\
\hline & Methanogenic & 0.02 & \\
\hline $\begin{array}{l}\text { Enrichment } \\
\text { culture }\end{array}$ & Iron(III)-reducing & $7.9 \pm 2.0$ & {$[32]$} \\
\hline $\begin{array}{l}\text { Enrichment } \\
\text { culture }\end{array}$ & Sulphate-reducing & 9.1 & {$[20]$} \\
\hline Field study & Methanogenic & 0.8 & {$[21]$} \\
\hline
\end{tabular}

\section{$\underline{\text { EtBE }}$}

Enrichment

culture

$$
\text { Methanogenic }
$$

Nitrate-reducing

3.8

${ }^{\mathrm{a}}$ AQDS = anthraquinone-2,6-disulfonate

\section{Anaerobic degradation of TBA}

TBA is frequently used as a solvent or fuel additive to increase the octane number for combustion. Furthermore, it has been suggested that TBA is a recalcitrant end product of anaerobic MtBE degradation [16], however, previous degradation studies have shown contradicting results. Several studies have shown slow anaerobic TBA degradation or no degradation at all [21, 32-34]. In contrast, mineralization of MtBE without accumulation of TBA has been shown under denitrifying conditions [31]. Anaerobic TBA degradation with iron(III) as electron acceptor has been demonstrated in microcosms at rates of up to $0.28 \% \mathrm{TBA} /$ day [35]. The same authors also found TBA degradation under methanogenic conditions $(0.16 \pm 0.04 \% \mathrm{TBA} /$ day $)$ and under sulphate reducing conditions $(0.16 \pm 0.04 \% \mathrm{TBA} / \mathrm{day}) . \mathrm{In}$ another study, no biodegradation of fuel oxygenates and TBA was observed after 80 days in microcosm experiments under manganese(IV), iron(III)- and methanogenic reducing conditions [36]. Bradley and others (2002) also did not find TBA degradation under methanogen- 
ic and iron(III)-reducing conditions, whereas under nitrate and manganese-reducing conditions $20-72 \%$ and $14-100 \%$ respectively, of TBA was found back after 198 days [37]. A detailed overview of studies with TBA degradation rates is given in Table 1.3.

Table 1.3 TBA degradation rates under anoxic conditions using nitrate, manganese(IV), iron(III), or sulphate as electron acceptors.

\begin{tabular}{|c|c|c|c|}
\hline $\begin{array}{c}\text { Type of exper- } \\
\text { iment }\end{array}$ & Condition & $\begin{array}{c}\text { TBA degra- } \\
\text { dation rate } \\
{[\mu \mathrm{M} / \text { day or }} \\
\text { stated other- } \\
\text { wise] } \\
\end{array}$ & References \\
\hline \multirow[t]{3}{*}{$\begin{array}{l}\text { Enrichment } \\
\text { culture }\end{array}$} & Methanogenic & $0.16 \pm 0.04 \%$ & {$[35]$} \\
\hline & Sulphate reducing & $0.16 \pm 0.04 \%$ & {$[35]$} \\
\hline & Iron(III)-reducing & $0.28 \%$ & {$[35]$} \\
\hline Field study & $\begin{array}{l}\text { Iron(III)-sulphate } \\
\text { reducing }\end{array}$ & $0.26-1.1$ year $^{-1}$ & {$[38]$} \\
\hline \multirow{2}{*}{$\begin{array}{l}\text { Enrichment } \\
\text { culture }\end{array}$} & Nitrate-reducing & $0.002-0.02$ & {$[37]$} \\
\hline & $\begin{array}{l}\text { Manganese(IV)- } \\
\text { reducing }\end{array}$ & $0.01 \pm 0.01$ & {$[37]$} \\
\hline $\begin{array}{l}\text { Enrichment } \\
\text { culture }\end{array}$ & Methanogenic & $0.07 \pm 0.02$ & {$[32]$} \\
\hline $\begin{array}{l}\text { Enrichment } \\
\text { culture }\end{array}$ & Methanogenic & $6.0 \pm 4.7$ & {$[21]$} \\
\hline
\end{tabular}

Anaerobic degradation of benzene

Anaerobic benzene degradation has been demonstrated in enrichment cultures [39-44] and pure cultures [45-47] under different redox conditions [48]. Dechloromonas strains RCB and JJ, two Azoarcus strains (DN11 and AN9) [46], a strain of Bacillus cereus [45] and Pseudomonas stutzeri strain BN [47] have been isolated for their ability to degrade benzene anaerobically with nitrate as electron acceptor. Alicycliphilus denitrificans strain BC has been shown to grow on benzene with chlorate as electron acceptor [49]. Recently, evidence for a prominent role of specific members of the family Peptococcaceae in anaerobic benzene degradation under denitrifying conditions was obtained using DNA-stable isotope probing of chemostat-derived biomass with ${ }^{13} \mathrm{C}$-labelled benzene $[43,50]$. The authors also suggested that in this consortium anaerobic benzene degradation proceeded via a syntrophic interaction. Many laboratory studies on benzene degradation have been performed over the 


\section{Chapter 1.}

years using different redox conditions (Table 1.4), however, the pathway of anaerobic benzene degradation is still not well understood. Three possible mechanisms have been proposed: (i) benzene hydroxylation, (ii) benzene carboxylation and (iii) benzene methylation $[48,51,52]$.

Table 1.4 Anaerobic benzene degradation rates (laboratory) in the presence of different electron acceptors or under methanogenic conditions.

\begin{tabular}{ccc}
\hline Condition & $\begin{array}{c}\text { Benzene degradation rate } \\
{[\boldsymbol{\mu M} \text { /day }]}\end{array}$ & References \\
\hline Chlorate-reducing & $4.2-1872$ & {$[43,53-55]$} \\
Nitrate-reducing & $0.004-66 \pm 11$ & {$[39,41,43-46,53,56-62]$} \\
Manganese(IV)-reducing & 0.83 & {$[63]$} \\
Iron(III)-reducing & $0.08-35.4$ & {$[43,61,63-67]$} \\
Sulphate-reducing & $0.09-52$ & {$[41,58,61,79-81]$} \\
Methanogenic & $0.25-75$ & {$[82]$} \\
Soil humic acids & 0.22 & \\
\hline
\end{tabular}

Anaerobic degradation of MtBE, EtBE, TBA and benzene in mixtures

Laboratory studies are often performed with individual substrates, but contaminated locations often contain mixtures of different compounds. In the research described in this thesis also a mixture containing the compounds of interest, i.e. MtBE, EtBE, TBA and benzene, will be used. In a mixture biodegradation of different fuel compounds, such as benzene, toluene, ethylbenzene and xylene (BTEX), may have different interactions. Most importantly, (1) the presence of one compound can stimulate the degradation of other compounds, (2) the presence of one compound can inhibit the degradation of other compounds, or (3) the presence of lower and higher compound concentrations can have a stimulatory or inhibitory effect, respectively, on the degradation of other compounds [83]. Cometabolic degradation may occur in mixtures which is a process in which two compounds are simultaneously degraded by microorganisms, and where the degradation of the secondary substrate depends on the presence of the primary substrate [84]. 
Biodegradation of biofuel components may inhibit the degradation of fossil-based fuel compounds such as benzene. For example, biodegradation of biofuel alcohols may produce metabolites at concentrations that render benzene degradation thermodynamically unfeasible under methanogenic conditions and therefore increase the plume length of benzene [85]. The biofuel ethanol is preferentially utilized as compared to BTEX compounds (e.g. benzene) and has a variable negative effect on BTEX degradation depending on the redox condition and aquifer material $[86,87]$. Besides this also a loss in electron acceptor may inhibit the degradation of contaminants in mixtures.

Previous studies indicate that MtBE had a negligible effect on benzene and ethanol degradation $[86,87]$. In turn, the presence of benzene and ethanol had no effect on MtBE degradation [88].

\section{Remediation technologies}

Since anaerobic degradation of contaminants without any intervention (natural attenuation) is often considered slow or absent, groundwater and soil are often actively remediated [89]. Soil and groundwater remediation techniques are based on physical (e.g. air sparging, filtration), chemical (e.g. chemical oxidation, photo catalysis) and/or biological processes (e.g. bioremediation, phytoremediation), which can be applied on itself or in combination [90], and which can be performed in situ or ex situ. In situ bioremediation is performed at the polluted location itself (below-ground in a polluted aquifer) while ex situ bioremediation takes place above-ground. The choice of bioremediation strategy depends on the location, type and extent of contamination and associated costs. Generally, ex situ techniques are more expensive compared to in situ techniques as a result of costs due to excavation and groundwater extraction [91]. There are three main bioremediation strategies: 1) monitored natural attenuation; intrinsic bioremediation, 2) stimulation of natural biodegradation; biostimulation and 3) augmentation of indigenous microbial communities with microorganisms able to degrade the contaminant; bioaugmentation.

Bioremediation is considered cost effective, sustainable and can accelerate the natural biodegradation powered by microbial enzymes [92]. In Figure 1.3 the most frequently applied remediation techniques for contaminated soil in different European countries, including the Netherlands, are visualized [93]. 


\section{Chapter 1.}

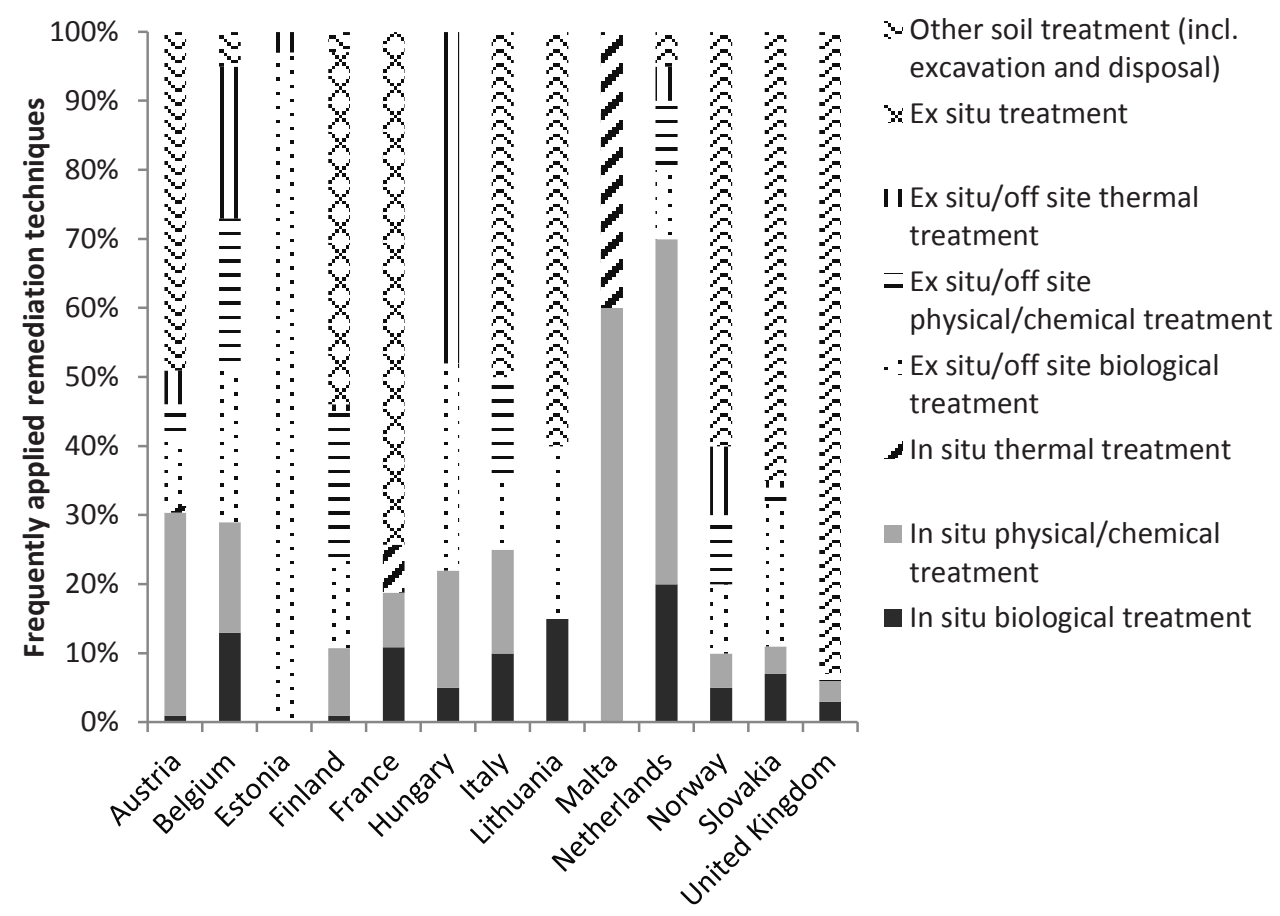

Figure 1.3 Most frequently applied remediation techniques for contaminated soil in different European countries [93].

To effectively remediate a contaminated site it is important to focus on all aspects considering physical, chemical and biological conditions and processes prevailing at a given location such as contaminant concentration, contaminant bioavailability and availability of nutrients [94]. The research described in this thesis focuses on in situ biostimulation and bioaugmentation.

\section{Biostimulation}

Biodegradation in soil can be limited by various factors, including availability of nutrients (e.g. electron acceptors, electron donor, and/or vitamins), temperature, contaminant bioavailability and $\mathrm{pH}$. In biostimulation studies, the indigenous microbial community is stimulated to increase the in situ degradation rate. Depending on the type of the contaminant, biostimulation involves the addition of electron acceptors (e.g. oxygen) and/or donors, and other macro- and micro-nutrients (e.g. nitrogen, phosphorous, vitamins) [95], adjustment of 
the $\mathrm{pH}$ or enhancing the contaminant bioavailability by using for example surfactants $[95$, 96].

\section{Bioaugmentation}

If the native microbial community at the contaminated site lacks a significant number of microorganisms capable of biodegradation of the contaminants, bioaugmentation can be applied. Bioaugmentation is the addition of specialized microorganisms at high concentrations to the contaminated site $[97,98]$. If bioaugmentation works, the use of this process at a contaminated location should result in smaller plume formation. Furthermore, bioaugmentation aims to increase the in situ degradation rate and decrease the total time needed for remediation. Several laboratory studies of bioaugmentation aiming to enhance anaerobic degradation of benzene were performed over the last decade (Table 1.5). To our knowledge, there are no experiments described in which microbial communities were bioaugmented to anaerobically degrade MtBE, EtBE or TBA.

Table 1.5 Anaerobic bioaugmentation studies of benzene contaminated soils. In all cases, experiments were performed with enrichments available in the lab (trial and error) rather than with site adjusted enrichments.

\begin{tabular}{|c|c|c|c|c|}
\hline Condition & Contaminant(s) & Inoculant & Biostimulation $^{\mathrm{a}}$ & Reference \\
\hline $\begin{array}{l}\text { Laboratory } \\
\text { test } \\
\text { (batch) }\end{array}$ & BTEX & $\begin{array}{l}\text { Enriched anaer- } \\
\text { obic BTEX- } \\
\text { adapted mixed } \\
\text { consortia }\end{array}$ & $\mathrm{NaNO}_{3}$ & {$[40]$} \\
\hline $\begin{array}{l}\text { Laboratory } \\
\text { test } \\
\text { (batch) }\end{array}$ & Benzene & $\begin{array}{l}\text { Azoarcus sp. } \\
\text { strain DN11 }\end{array}$ & $\mathrm{NaNO}_{3}$ & {$[59]$} \\
\hline $\begin{array}{l}\text { Laboratory } \\
\text { test (col- } \\
\text { umn) }\end{array}$ & BTEX & $\begin{array}{l}\text { Enriched anaer- } \\
\text { obic benzene- } \\
\text { adapted meth- } \\
\text { anogenic consor- } \\
\text { tium }\end{array}$ & $\mathrm{NO}_{3}{ }^{-}, \mathrm{SO}_{4}{ }^{2-}$ & [99] \\
\hline $\begin{array}{l}\text { Laboratory } \\
\text { test } \\
\text { (batch) }\end{array}$ & Benzene & $\begin{array}{l}\text { Benzene degrad- } \\
\text { ing sulphate- } \\
\text { reducing organ- } \\
\text { isms }\end{array}$ & $\mathrm{Na}_{2} \mathrm{SO}_{4}$ & [74] \\
\hline
\end{tabular}




\section{Chapter 1.}

Efficient bioaugmentation can be performed when a contaminated site does not contain sufficient numbers of microorganisms able to effectively degrade the contaminant. The process of bioaugmentation involves five steps (Figure 1.4):

1. Characterization of the site and obtaining a site adjusted enrichment

2. Cultivation of the microbial biomass

3. Inoculation of the microorganisms

4. Establishment of inoculum in the local ecosystem and biostimulation

5. Monitoring bioremediation progress

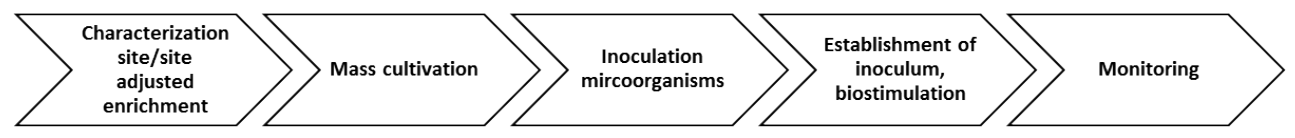

Figure 1.4 Steps involved in the process of bioaugmentation.

Step 1: Characterization of the site and obtaining a site adjusted enrichment

The first step of the bioaugmentation procedure is to characterise the site and to obtain a site adjusted enrichment. Site adjusted enrichments can be obtained by growing native microorganisms in the lab (exogenously) with appropriate amendments [100]. To date, all anaerobic benzene degrading bioaugmentation studies considered in Table 1.5 have been trial-and-error/empirical studies, where enrichments available in the laboratory turned out to result in degradation after inoculation of these enrichments using laboratory experiments. It can be anticipated, however, that for a more effective bioaugmentation strategy, it may be essential to go beyond such trial-and-error approaches and to systematically perform and study the process of bioaugmentation to substantiate the potential of this remediation technique. Generally, enrichments are obtained by adding environmental samples to batch cultures. The conditions in these cultures normally differ from prevailing environmental conditions, and batch enrichments will in general select for the fastest growing organisms [101], whereas in the environment other microbes compared with the selected fast growing species usually dominate. The most predominant species might be obtained by employing dilution-to-extinction enrichments when the optimal growth conditions are met. It should be noted, however, that in many cases microorganisms resist cultivation under laboratory conditions [102]. To this end, continuous culturing approaches can be used to provide more 
environmentally relevant conditions and can be considered more suited for site adjusted enrichment.

\section{Step 2: Cultivation of the microbial biomass}

Cultivation of contaminant degrading organisms can be done in the laboratory, using simple batch cultures, continuous culture systems, or by using on site reactors. To provide the biomass required for full scale field applications, larger reactors can be used, and a higher density of the organisms might be obtained when physico-chemical parameters can be adjusted to enhance growth of biodegrading microbes. Further experiments are required to determine exactly how to cultivate the mass most optimal for inoculation in the field. Furthermore, novel cultivation techniques such as an isolation chip based on in situ highthroughput cultivation of environmental microorganisms are needed for slow growing organisms to speed up the process [103].

\section{Step 3: Inoculation of microorganisms}

Distribution of the augmented microorganisms in the aquifer can be difficult due to sorption of microbes by soil particles [97]. Different techniques have been investigated to increase the distribution of the augmented microorganisms, including the use of adhesion-deficient bacteria, ultramicrobacteria, surfactants, encapsulation, and immobilization [97, 98]. Megharaj and others (1997) suggested that bacteria pre-adapted to the environmental conditions may survive better and degrade the contaminant more efficiently compared with nonpre-adapted bacteria [104]. This suggestion of pre-adaptation has been proven with Sphingomonas wittichii RW1 decomposing polychlorinated dibenzo-p-dioxins [105]. Fluctuations in environmental conditions such as temperature fluctuations have been suggested to increase the ability of microorganisms to invade novel environments [106, 107].

All bioaugmentation studies listed in Table 1.5 have been described as being successful in biodegradation of the contaminants. Nevertheless, many studies do not show whether the inoculants remain active, grow or were responsible for biodegradation of the contaminant. Therefore it cannot be excluded that 'successful' studies are due to growth and activity of members of the indigenous community in response to the addition of nutrients, acceptors or substrates supplied together with the cultivated degrading microorganisms. It is also conceivable that the genetic material of the inoculants can be taken up by 


\section{Chapter 1.}

members of the indigenous community via horizontal gene transfer enabling the recipients to degrade the contaminants. This process might in fact be considered as gene bioaugmentation. For example, reductive dehalogenase genes have been shown to be often embedded in genomic islands in high plasticity regions of the genomes of organohalide respiring microorganisms, suggesting horizontal transfer could occur [108]. Furthermore, it was previously suggested that horizontal gene transfer and genetic recombination of existing genes between indigenous microorganisms were responsible for the evolution of the catabolic pathway [109]. Seeding of a polluted environment with mobile genetic elements and/or organisms carrying these elements that are horizontally transferred can be considered as a bioaugmentation technique. However, it is necessary to investigate whether horizontal gene transfer actually takes place or/and whether the inoculated organisms themselves degrade the contaminant to obtain the most optimal biodegradation results by using bioaugmentation.

Step 4: Establishment of inoculum in the local ecosystem and biostimulation Appropriate controls, focused on the difference between inoculation of washed cells, inoculant with medium, and exclusively medium (no cells), and extensive monitoring have to be included to demonstrate the success of inoculation with microorganisms and evaluate its benefits. To our knowledge, these control conditions are frequently omitted in bioaugmentation studies, and thus further research is required in order to evaluate the difference between stimulation of indigenous microorganisms and biodegradation by added microorganisms.

The effectiveness of biodegradation depends on the interactions between the microorganisms, the environment, and the contaminants (Figure 1.5). 


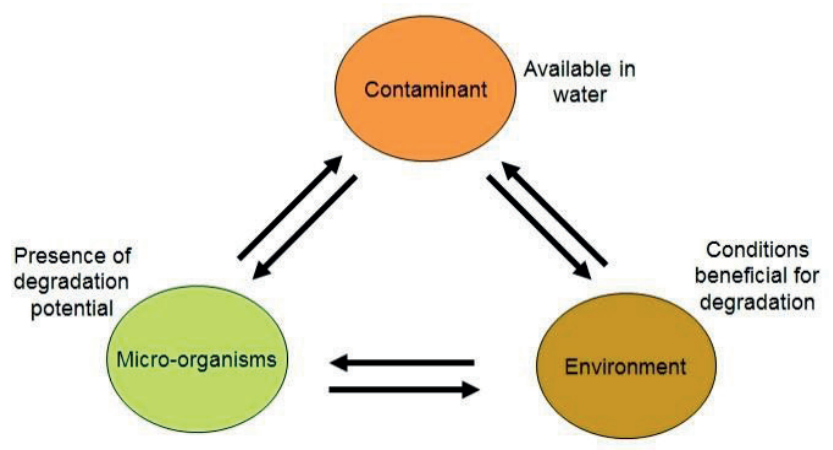

Figure 1.5 The interaction between micro-organisms, the abiotic environment, and the contaminants.

The interactions between these three components are well described [98, 110]. Obviously, the environmental conditions should sustain the activity of the microorganisms. Therefore, it is crucial that, in addition to the augmentation with microbial populations adapted to the environmental conditions, the circumstances for them to thrive are optimized. Consequently, all elements of the triad microorganisms, contaminant and environment should be optimized to increase the chance of biodegradation through bioaugmentation. Bioavailability of the contaminants can be optimized by, for example, pumping and mixing of groundwater, or supply of (bio)surfactants. A beneficial environment can be created by supply of proper electron donors (e.g. organic substrates), electron acceptors (e.g. oxygen, nitrate, chlorate or sulphate), other nutrients (e.g. nitrogen, sulphur, phosphorous or vitamins), by optimizing the $\mathrm{pH}$ or temperature, etc.

\section{Step 5: Monitoring bioremediation progress}

The process of bioaugmentation needs to be monitored intensively (depending on the location) to observe and confirm the potential of using this technique in the field (e.g. redoxconditions, what happens with the inoculated microorganisms and the degradation of the contaminant(s)). The inoculants can be monitored with for example Real-Time PCR (qPCR) using primers specifically targeting the (different) microbial populations that are included. The degradation of the contaminant(s) can be followed using analytical methods such as gas chromatography coupled with flame ionization detection and mass spectrometry (GCFID and GC-MS). The use of nutrients and electron acceptors can be followed using liquid chromatography. 


\section{Chapter 1.}

To conclude, studies of bioaugmentation on benzene have been done in the past using simple laboratory culture systems. However, to date little attention has been paid to apply bioaugmentation to enhance anaerobic degradation of MtBE, EtBE, TBA and benzene at a relevant scale (i.e. column studies or field tests). Kasai et al. (2007) found a decrease of the augmented strain DN 11 of Azoarcus sp. after depletion of benzene [59]. Strain DN 11 complemented the indigenous community. However, it is also possible that the augmented consortia can outcompete the indigenous community. Therefore, the scientific basis of the bioaugmentation potential remains rather weak and needs further substantiation. The gap between laboratory and field studies can be bridged by scale-up soil column experiments, continuous culture experiments and on site pilot tests.

\section{Aim and outline of this thesis}

The aim of the research described in this thesis was to gain more insight in biodegradation of several 'contaminants of the future' using biostimulation (different nutrients and electron acceptors) and bioaugmentation with laboratory-cultivated microbial communities. Laboratory experiments using MtBE, EtBE, TBA and benzene were performed to obtain pure cultures or consortia of microorganisms anaerobically degrading these contaminants. These organisms were then studied and cultivated to perform bioaugmentation tests using batch experiments and continuous culture experiments. Through this study, we aimed to gain knowledge about ecology and physiology of the microorganisms involved in the anaerobic degradation of MtBE, EtBE, TBA and benzene and their potential use for biostimulation and bioaugmentation. Research chapters 2, 3 and 4 describe the biodegradation using batch and continuous culture experiments. The last research chapter, Chapter 5, describes the potential of bioaugmentation on-site.

In Chapter 2, the activity and microbial community composition of a benzene degrading, denitrifying continuous culture is described. An anaerobic continuous biofilm culture grown for more than 14 years on benzene with nitrate was used to determine steady state degradation rates, microbial community composition dynamics in the biofilm, and the initial anaerobic benzene degradation reactions. 
The anaerobic degradation of MtBE, EtBE, TBA and benzene in a mixture under different redox conditions is described in Chapter 3. We also studied the effect of growth substrate analogues on the degradation of MtBE, EtBE and TBA.

The micro-oxic degradation of EtBE using both algae and bacteria is described in

\section{Chapter 4.}

Chapter 5 describes the potential of using the benzene degrading, denitrifying continuous culture described in Chapter 2 for bioaugmentation purposes. The microbial community was cultivated in the laboratory and several augmentation experiments with this culture were performed. In addition, several pure cultures capable of benzene degradation with nitrate were isolated. The possible role of horizontal gene transfer enabling anaerobic degradation of benzene is suggested.

In Chapter 6, the main conclusions of this thesis and the relevance of these findings are summarized and discussed in a broader perspective. 
Chapter 1. 


\section{Chapter 2. Benzene degradation in a denitrifying biofilm}

\section{reactor: activity and microbial community composition}

Marcelle J. van der Waals, Siavash Atashgahi, Ulisses Nunes da Rocha, Bas M. van der Zaan, Hauke Smidt and Jan Gerritse

This chapter has been published as:

Benzene degradation in a denitrifying biofilm reactor: activity and microbial community composition, Applied Microbiology and Biotechnology, 2017 101:12 p. 5175-5188 Marcelle J. van der Waals, Siavash Atashgahi, Ulisses Nunes da Rocha, Bas M. van der Zaan, Hauke Smidt and Jan Gerritse (2017) 


\title{
Chapter 2.
}

\begin{abstract}
Benzene is an aromatic compound and harmful for the environment. Biodegradation of benzene can reduce the toxicological risk after accidental or controlled release of this chemical in the environment. In this study, we further characterised an anaerobic continuous biofilm culture grown for more than 14 years on benzene with nitrate as electron acceptor. We determined steady state degradation rates, microbial community composition dynamics in the biofilm and the initial anaerobic benzene degradation reactions. Benzene was degraded at a rate of $0.15 \mu$ mol.mg protein ${ }^{-1}$.day $^{-1}$ and a first order rate constant of 3.04 day $^{-1}$ which was four-fold higher than rates reported previously. Bacteria belonging to the Peptococcaceae were found to play an important role in this anaerobic benzene-degrading biofilm culture, but also members of the Anaerolineaceae were predicted to be involved in benzene degradation or benzene metabolite degradation based on Illumina MiSeq analysis of $16 \mathrm{~S}$ ribosomal RNA genes. Biomass retention in the reactor using a filtration finger resulted in reduction of benzene degradation capacity. Detection of the benzene carboxylase encoding gene, $a b c A$, and benzoic acid in the culture vessel indicated that benzene degradation proceeds through an initial carboxylation step.
\end{abstract}

\section{Introduction}

Benzene is a constituent of crude oil and gasoline and is often considered as riskdetermining compound at contaminated locations, because it easily dissolves and transports in groundwater and is carcinogenic [11]. Benzene may enter the groundwater from for example leaking storage tanks or pipelines. Benzene is readily biodegradable under aerobic conditions [111]. However, once contaminants such as benzene reach the groundwater, oxygen is usually depleted by microbial respiration [112]. Consequently, anaerobic degradation is essential for benzene removal from such oxygen-depleted subsurface environments. The biggest challenge for anaerobic benzene biodegradation is to overcome the compound's extreme chemical stability in the absence of molecular oxygen. Three mechanisms for the initial step of anaerobic benzene degradation have been proposed: (1) benzene hydroxylation, (2) benzene carboxylation, and (3) benzene methylation, leading to the formation of respectively phenol, benzoate and toluene as the main metabolites $[48,51,52]$.

Benzene degradation under nitrate-reducing conditions has been demonstrated in enrichments [39-44, 61] and pure cultures [45]. Dechloromonas strains RCB and JJ [46], two 
Azoarcus strains (DN11 and AN9) [44] and Bacillus cereus [45] have been isolated for their ability to degrade benzene with nitrate as electron acceptor. The genome of Dechloromonas strain RCB does not include the genes encoding for benzylsuccinate synthase or enzymes involved in the benzoyl-CoA pathway for monoaromatics [113]. The isolated strains might therefore degrade benzene through intra-aerobic pathways i.e. using oxygen released from nitrogen reduction. Hence, to the best of our knowledge, there has been no report on the isolation of strictly anaerobic denitrifying benzene degraders. Recently, bacteria related to the anaerobic Gram-positive Peptococcaceae have been shown to play an important role in anaerobic benzene degradation under denitrifying [43, 114], ironreducing $[50,115]$ and sulphate-reducing conditions $[43,69,70,78,116]$.

Microbial community dynamics can be efficiently studied using a combination of generic high-throughput analysis of $16 \mathrm{~S}$ ribosomal RNA (rRNA) genes through next generation technology sequencing (e.g. Illumina MiSeq) of PCR-barcoded amplicons, and quantitative-PCR (qPCR) targeting 16S rRNA genes of specific microbial taxa or genes involved in key catabolic reactions (e.g. benzene degradation). However, only a limited number of qPCR assays targeting key catabolic genes are currently available. Available functional gene qPCR assays for the degradation of benzene and potential main metabolites target benzylsuccinate synthase $\alpha$-subunit ( $b s s A$ ) involved in anaerobic toluene degradation [117], and 6-oxocyclohex-1-ene-1-carbonyl-coA hydrolase (bamA) [118], involved in the reductive benzene ring cleavage. A qPCR assay targeting benzene carboxylase genes that could give information on benzene degradation through the proposed carboxylation pathway is currently not available [119].

We studied an anaerobic benzene-degrading microbial consortium, which has been enriched in a continuous culture with nitrate as electron acceptor for more than 14 years. Over these years, microbial biofilms have formed on the glass and metal surfaces within the reactor vessel. Besides nitrate, this consortium is able to couple benzene degradation to reduction of chlorate, ferric iron or sulphate as electron acceptors [43]. From previous studies it is known that after many years of enrichment, still a diverse microbial community is present in the continuous culture vessel [43]. DNA-stable isotope probing with ${ }^{13} \mathrm{C}$-labelled benzene indicated that members of the Peptococcaceae are dominant benzene degraders presumably involved in the initial benzene ring activation. Recently, bacteria belonging to 


\section{Chapter 2.}

the Ochrobactrum sp. and Bacillus sp. possibly capable of anaerobic benzene degradation with nitrate as electron acceptor were isolated from the same continuous culture (Balk, personal communication). For effective benzene bioremediation practices in the field, detailed studies on the degradation capabilities and mechanisms of the anaerobic benzene degrading organisms are necessary. To that end, the objectives of this study were: (i) to determine the maximum benzene degradation capacity of this consortium under a variety of controlled continuous culture conditions, (ii) to obtain a highly active benzene degrading community, (iii) to characterise differences in the microbial composition in the biofilm and liquid phase of the reactor and activity of the microbial populations involved, and (iv) to provide further mechanistic insight in the initial step in benzene degradation.

\section{Materials and Methods}

The anaerobic benzene degrading microbial community is found predominantly attached to the glass and metal surfaces of the culture vessel submerged in the growth medium. Originally, the continuous culture was inoculated with soil from a benzene-contaminated site located in the northern part of the Netherlands [43]. The experiments were done with the microbial community in the continuous culture before and after the addition of a filtration finger in an attempt to further increase the activity of the benzene-degrading culture. Continuous culture experiments were performed to obtain the first-order benzene degradation rate constant, $\mathrm{k}$, and the specific benzene degradation rate, $\mathrm{r}_{\max }$, of the microbial community. The composition of the communities residing in the reactor was studied by molecular techniques. LC-qTOF-MS measurements were used to reveal the anaerobic benzene degradation mechanism.

\section{Continuous culture}

The anaerobic continuous culture was operated for more than 14 years on benzene as the sole electron donor under nitrate-reducing conditions as described previously [43]. The system was adapted for this study as follows: (i) the influent medium contained per liter: $0.5 \mathrm{~g} \mathrm{KH}_{2} \mathrm{PO}_{4}, 0.25 \mathrm{~g}\left(\mathrm{NH}_{4}\right)_{2} \mathrm{SO}_{4}, 0.4 \mathrm{~g} \mathrm{NaNO}_{3}, 10 \mathrm{ml}$ trace element solution SL-4 (DSMZ medium 462, www.dsmz.de), $1 \mathrm{ml}$ trace element solution SL+ (contained per liter: $0.5 \mathrm{~g}$ $\mathrm{NaOH}, 3 \mathrm{mg} \mathrm{Na} \mathrm{SeO}_{3}, 4 \mathrm{mg} \mathrm{Na} \mathrm{WO}_{4}$ ); (ii) the $\mathrm{pH}$ of the medium was 6.7; (iii) the temperature in the culture vessel was maintained at $25^{\circ} \mathrm{C}$ and the liquid phase was stirred at $200 \mathrm{rpm}$; (iv) the headspace was flushed with $\mathrm{N}_{2} / \mathrm{CO}_{2}(80 \% / 20 \%)$ and the gas flowrate 
was kept proportional (2.4 fold higher) to the medium pump rate using a Marprene pump tube (Watson-Marlow, MA, USA). The medium was kept under a $\mathrm{N}_{2} / \mathrm{CO}_{2}(80 \% / 20 \%)$ atmosphere and added via viton tubing (Rubber BV, Hilversum, the Netherlands) and a PVC pump tube (Watson-Marlow). Benzene was injected from a $22 \mathrm{mM}$ anaerobic aqueous stock solution directly into the liquid phase using a syringe pump (KDS-230-CE, kdScientific, MA, USA) and viton tubing (Rubber BV). The mixed $\mathrm{N}_{2} / \mathrm{CO}_{2}$ gas was passed over a $460 \mathrm{ml}$ glass column filled with hot copper filings $\left(250^{\circ} \mathrm{C}\right)$ to remove traces of oxygen before entering the growth vessel. The benzene degradation rate constant was obtained by changing the dilution rate, i.e. by changing the pump rates of the medium and benzene proportionally. The specific benzene degradation rate was calculated using the total protein content in the culture vessel in both the liquid and biofilm. To determine whether benzene degradation was diffusion limited in the biofilm in the culture vessel, the stirring rate of the reactor was varied between 100 and $200 \mathrm{rpm}$.

Transfers of liquid with aggregates of biofilm from the continuous culture to microcosms were supplied with $100 \mu \mathrm{M}$ benzene and $3.6 \mathrm{mM}$ nitrate to obtain microcosm enrichments. To test growth on benzoate, $10 \%(\mathrm{v} / \mathrm{v})$ liquid from the reactor was transferred to microcosms with $10 \mathrm{mM}$ benzoate and $40 \mathrm{mM}$ nitrate.

\section{Retentostat experiment}

A retentostat system with a filtration finger was used in an attempt to further increase the activity of the benzene degrading culture by retaining biomass in the reactor vessel. To this end, a bottle with filtration finger [120, 121] (VU University, Molecular Cell Physiology, Amsterdam, the Netherlands, cellulose acetate filter; $0.22 \mu \mathrm{m}$, Millipore, MA, USA) was added to the system (Figure 2.1). Liquid was recirculated between the reactor vessel and the bottle with the filtration finger. Approximately $10 \%$ of the recirculating liquid was pumped through the finger to the effluent vessel. 


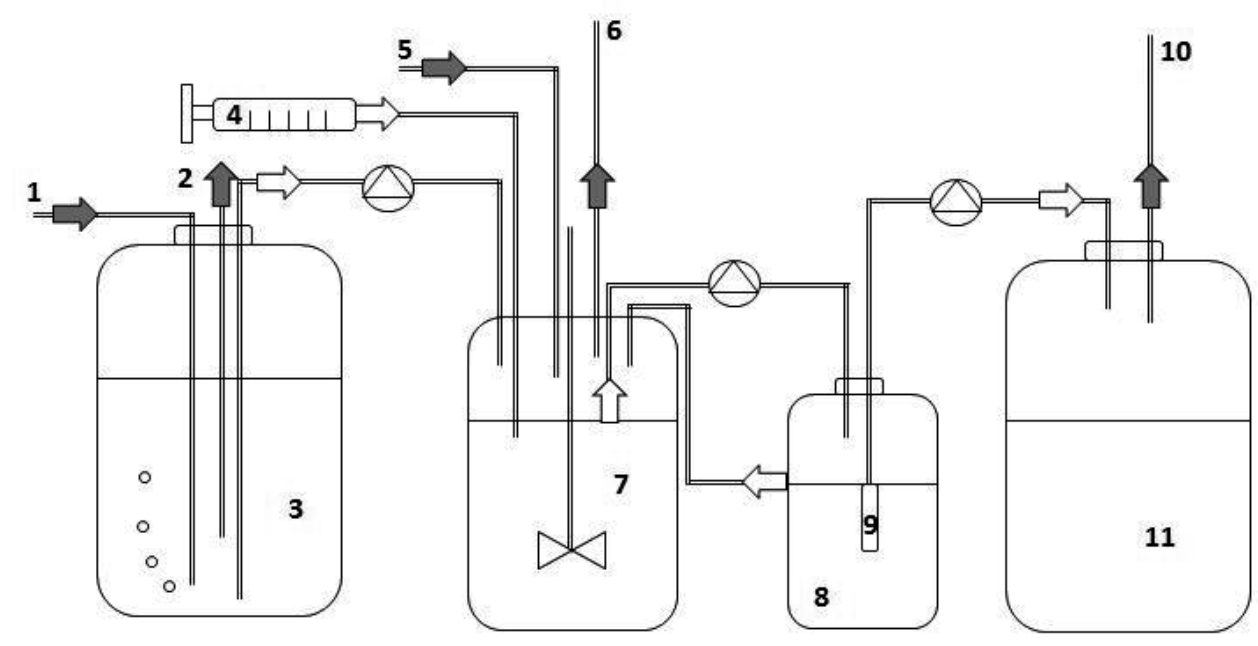

Figure 2.1 Schematic presentation of experimental set up of the reactor vessel with retentostat finger. The medium (3) kept under $\mathrm{N}_{2} / \mathrm{CO}_{2}\left(1\right.$ and 2) and benzene (4) were continuously added to the $\mathrm{N}_{2} / \mathrm{CO}_{2}$ flushed (5 and 6) continuous culture (7). The recirculation batch (8) with retentostat finger (9) was used to filter $(0.22 \mu \mathrm{m}$ pore size $)$ the liquid phase pumped to the effluent tank (11). Gas is released from the effluent tank directly to the atmosphere (10). Due to the pressure difference liquid is flowing back from the recirculation batch to the continuous system.

\section{Analyses}

Benzene was measured with a Varian 3800 gas chromatographic (GC) system equipped with a flame ionization detector (FID), a CP-PoraBond Q column $(0.32 \mathrm{~mm}$ x $25 \mathrm{~m})$ and a retention gap (methyl deactivated, $320 \mu \mathrm{m}$ ID) (Varian, Middelburg, the Netherlands). The FID detector was set at $300^{\circ} \mathrm{C}$, and the sample was injected at $200^{\circ} \mathrm{C}$ with a split ratio of $5: 1$. The oven temperature program was $3 \mathrm{~min}$ at $50^{\circ} \mathrm{C}$, followed by an increase of $10^{\circ} \mathrm{C} / \mathrm{min}$ to $250^{\circ} \mathrm{C}$ for $2 \mathrm{~min}$. The flow rate of helium carrier gas was $2 \mathrm{ml} / \mathrm{min}$. Continuous culture headspace samples of $1 \mathrm{ml}$ were taken with a 1-ml Pressure-Lock gas syringe (Alltech, Breda, the Netherlands) followed by $0.5 \mathrm{ml}$ injection into the GC-FID. The benzene concentration in the liquid is expressed in $\mu \mathrm{M}$ assuming complete dissolution of benzene. For calibration six standards ranging from 10 to $200 \mu \mathrm{M}$ benzene were prepared in serum bottles with the same headspace/water ratio (v/v) as in the continuous culture. The bottles were crimp sealed with viton stoppers and aluminium caps. The liquid and headspace were flushed with $\mathrm{N}_{2} / \mathrm{CO}_{2}(80 \% / 20 \%)$. The coefficient of variation of this GC 
method was $5 \%$, and the benzene detection limit was $0.1 \mu \mathrm{M}$. Benzoate was measured using a LC-qTOF-MS as performed previously [122]. Liquid samples were conserved with $25 \mathrm{mg} / 1$ mercury chloride before LC-qTOF-MS analysis.

Continuous culture biofilm and liquid samples were analysed for protein content at a dilution rate of 2 day $^{-1}$, according to the procedure of Lowry et al. ${ }^{123}$. Six standards ranging from 0 to $500 \mu \mathrm{g}$ protein/ml of bovine serum albumin (BSA) were used for calibration. The total amount of biofilm protein in the culture vessel was assessed by extrapolating the amount of protein released from defined surface areas as described below to the total area of biofilm covered surfaces in the system.

\section{Biofilm sampling}

Continuous culture samples for analyses of changes in microbial composition and activity (nucleic acids and protein) were taken by scraping off defined areas of biofilm attached to the glass wall under a constant $\mathrm{N}_{2} / \mathrm{CO}_{2}(80 \%$ / 20\%) flow. Subsequently, the liquid phase in the vessel was stirred for $5 \mathrm{~min}$ at $200 \mathrm{rpm}$ to disperse the biofilm aggregates. Samples containing biofilm aggregates were taken directly from the liquid phase of the culture vessel using a $60 \mathrm{ml}$ syringe via a sampling pipe and viton tubing (Rubber BV). The samples were immediately stored at $-80^{\circ} \mathrm{C}$ until DNA extraction. Different areas of biofilm with different morphology, respectively white and brown biofilm, were sampled (Figure S2.1). Sample_1 contained $6.25 \mathrm{~cm}^{2}$ white biofilm (11\% biofilm and $89 \%$ liquid protein). Sample_2 contained also $6.25 \mathrm{~cm}^{2}$ white biofilm, corresponding to $11 \%$ biofilm and $89 \%$ liquid protein (sample taken 3 days later compared with Sample_1). Samples 3 and 4 contained $22.25 \mathrm{~cm}^{2}$ white and $4 \mathrm{~cm}^{2}$ brown biofilm corresponding to $70 \%$ biofilm and $30 \%$ liquid protein and $22.25 \mathrm{~cm}^{2}$ white and $8 \mathrm{~cm}^{2}$ brown biofilm corresponding to $100 \%$ biofilm, respectively.

\section{DNA extraction, 16S rRNA gene amplicon sequencing and data analysis}

Total DNA was extracted from the biofilm samples using a CTAB/phenol-chloroform method as described previously [124]. Illumina MiSeq amplicon sequencing of the extracted DNA was used for bacterial community analysis. Barcoded amplicons from the V1-V2 region of $16 \mathrm{~S}$ rRNA genes were generated using a two-step PCR method as described earlier [125]. In brief, the first amplification of partial bacterial 16S rRNA genes was done using primer pair 27F-DegS and 338R-I+II in $50 \mu \mathrm{l}$ PCR reactions. The primers were 5'- UniTag- 


\section{Chapter 2.}

extended [126]. The second amplification was done using $85 \mu 1$ mastermix, $10 \mu 1$ barcoded primers targeting the UniTag extensions and $5 \mu$ PCR product from the first PCR. Afterwards, barcoded PCR products were cleaned using the HighPrep PCR clean-up system (Magbio Genomics, MD, USA), quantified using Qubit (Thermo Fisher Scientific, MA, USA) and pooled in equimolar amounts as previously described [125]. Samples were sequenced by GATC (GATC Biotech AG, Constance, Germany) on a MiSeq platform. 16S rRNA amplicon sequence analysis was done using NG-Tax [127]. Briefly, paired-end libraries were filtered to retain read pairs with perfectly matching barcodes. 16S rRNA gene sequences were clustered into operational taxonomic units (OTUs) at $>98.5 \%$ sequence similarity. OTUs were assigned using an open reference approach and a customized SILVA 16S rRNA database version 111 [128]. The phylogenetic diversity (PD whole tree) was calculated using Quantitative Insights Into Microbial Ecology (QIIME) [129]. Quality filtering of the sequences was done using defined 'Mock' communities as a positive control to deal with issues associated with filtering parameter optimization [127]. Two different Mock communities used in this analysis had a Pearson correlation at genus level of 0.73 and 0.78 , respectively, with respect to the microbial community theoretically present in the Mock community, which are values routinely observed in other runs using the same set-up. Nucleotide sequence data reported are available at the European Nucleotide Archive (ENA) under accession number PRJEB18709.

\section{Quantification of total bacterial 16S rRNA genes, $\operatorname{bam} A$ and $b s s A$ genes}

An overview of the used primer pairs, probes and thermal cycling conditions for total bacterial 16S rRNA, bssA and bamA genes is shown in Table 2.1. Amplification of total bacterial $16 \mathrm{~S}$ rRNA genes was carried out in a $25 \mu \mathrm{l}$ reaction mixture containing $0.6 \mu \mathrm{M}$ of each primer, $3 \mu 1$ template DNA, $6.5 \mu 1$ MilliQ and $12.5 \mu 1$ 2x IQ SYBR Green Supermix (BioRad). The lower detection limit of the assay was 53.7 gene copies/ $\mu 1$ sample defined at the amount of cycles at the lowest detected calibration sample plus one cycle. Amplification of bam $A$ genes was performed in a $25 \mu \mathrm{l}$ PCR reaction mixture as described for the total bacterial 16S rRNA gene assay with the addition of $0.4 \mu \mathrm{g} / \mu \mathrm{l}$ BSA. Both assays were performed on a CFX96 Real-time PCR machine (Bio-Rad). The lower detection limit was 6.3 gene copies/ $\mu 1$ sample. Amplification of $b s s A$ genes was done with the addition of a Taqman FAM probe [130]. The lower detection limit of the bssA gene assay was 3.1 gene copies/ $\mu$ l sample. PCR amplification was carried out on an IQ5 real-time PCR system (Bio- 
Rad) using $0.6 \mu \mathrm{M}$ of each primer, $3 \mu 1$ template DNA, $6.3 \mu 1 \mathrm{MilliQ}, 12.5 \mu 1$ 2x IQ Supermix (Bio-Rad) and $0.08 \mu \mathrm{M}$ probe (Eurofins MWG Operon, Ebersberg, Germany). 
Chapter 2.

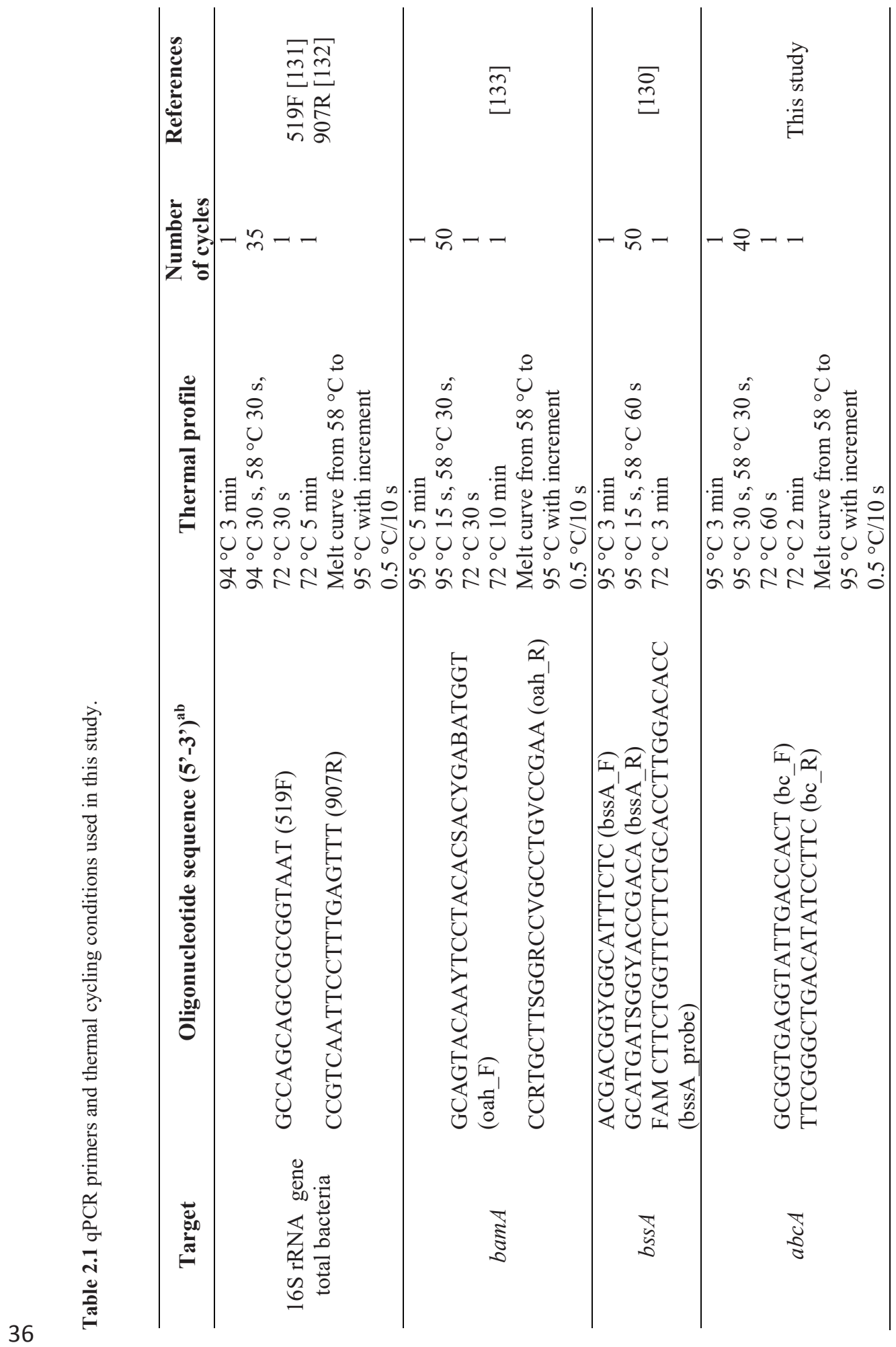


Benzene degradation in a denitrifying biofilm reactor

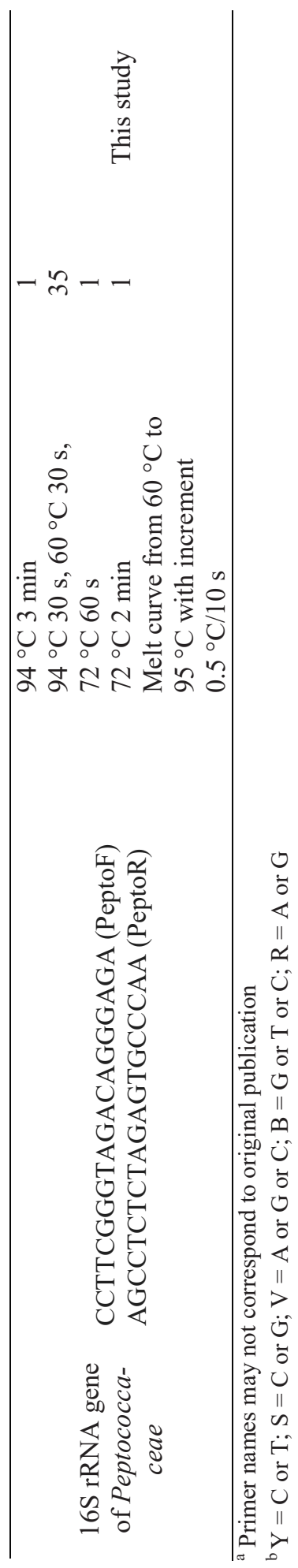




\section{Chapter 2.}

\section{Benzene carboxylase gene $(a b c A)$ qPCR primer design and calibration curve}

Amplification of the benzene carboxylase gene was done by designing a specific primer pair for $a b c A$, a gene described by Abu Laban et al. (2010) . The previously designed primer pair bc_F and bc_R (Table 2.1) $[134,135]$ was evaluated in silico using the publicly accessible NCBI BLAST search tool [136]. Reactor DNA material was used as template DNA to obtain an amplicon of 175 base pairs. Amplification was carried out in 40 cycles (30 sec at $95^{\circ} \mathrm{C}, 30 \mathrm{sec}$ at $58^{\circ} \mathrm{C}$ and $60 \mathrm{sec}$ at $72^{\circ} \mathrm{C}$ ) with an initial denaturation of $3 \mathrm{~min}$ at $95^{\circ} \mathrm{C}$ and a final elongation step of 2 min at $72^{\circ} \mathrm{C}$ on a CFX96 Real-time PCR system (BioRad) using TaKaRa Ex Taq polymerase according to the manufacturer's protocol (TAKARA BIO INC, Otsu, Japan). The amplicons were separated on a 1\% agarose gel and extracted using a QIAquick Gel Extraction Kit (Qiagen, Hilden, Germany) according to the manufacturer's protocol. Gene fragments were cloned into PCR2.1 TOPO vector using the TOPO cloning kit (Invitrogen, Breda, the Netherlands) according to the manufacturer's protocol with $100 \mu \mathrm{g} / \mathrm{ml}$ ampicillin and $32 \mu \mathrm{l}$ of $50 \mathrm{mg} / \mathrm{ml} \mathrm{X}$-gal. After growth, cells were pelleted, and plasmid was purified according to the manufacturer's protocol of the QIAprep miniprep system (Qiagen) and sequenced by Eurofins MWG Operon with M13 rev (-29) primer. The obtained sequence was $99 \%$ similar to the $a b c A$ gene sequence identified previously [115]. The DNA concentration of the cloned $a b c A$ gene amplicon was determined on a Nanodrop 1000 spectrophotometer (Isogen, De Meern, the Netherlands) to calculate the concentration of the gene copy numbers based on the molecular weight of doublestranded amplicons [137]. The stock solution was diluted to $10^{10}$ gene copies/ $\mu 1$ in MilliQ. The 8-point standard curve was optimized with a lower detection limit of 11.2 gene copies/ $\mu 1$ sample. Amplification of the $a b c A$ gene was done in a $25 \mu 1$ reaction mixture as described for bamA genes. The thermal amplification profile is shown in Table 2.1.

\section{Peptococcaceae 16S rRNA gene qPCR primer design and calibration curve}

Amplification of Peptococcaceae 16S rRNA genes was done using specific primers that were newly designed as described in da Rocha [138]. The specificity of primer pair peptoF and peptoR (Table 2.1) was evaluated in silico using the public accessible NCBI BLAST search tool, and the predicted amplicon size was 136 bp [136]. Reactor DNA material was used as template DNA. The standard curve was optimized using a CFX96 Real-time PCR (Bio-Rad) with a lower detection limit of 10.5 gene copies/ml sample. Amplification of 
Peptococcaceae 16S rRNA genes was done in a $25 \mu 1$ reaction mixture as described for the total bacterial 16S rRNA genes. The thermal cycling conditions are described in Table 2.1.

\section{Calculations}

First-order degradation rate constants $(k)$ were calculated as described previously according to Equation 2.1 [111].

$\mathrm{k}=\left(\frac{\log \left(\mathrm{x}_{1}\right)-\log \left(\mathrm{x}_{2}\right)}{\mathrm{t}_{2}-\mathrm{t}_{1}}\right)+\mathrm{D}$

where $t_{2}-t_{1}$ is the time interval in days. $x_{1}$ is the benzene concentration at time $t_{1}$ and $x_{2}$ is the benzene concentration at time $t_{2}$. $\mathrm{D}$ is the dilution rate of the continuous culture per day.

The specific degradation rate $(r)\left[\mathrm{mg}\right.$ benzene.mg protein ${ }^{-1}$. day $\left.{ }^{-1}\right]$ was calculated according to Equation 2.2:

$\mathrm{r}=\frac{\mathrm{I}_{\mathrm{b}}}{\gamma}$

where $\mathrm{I}_{\mathrm{b}}$ is the supply of benzene into the liquid phase of the continuous culture in $\mathrm{mg}$ benzene.day ${ }^{-1}$ and $\gamma$ is the protein content in the culture vessel in $\mathrm{mg}$.

The benzene reservoir concentration in the continuous culture vessel $\left(C_{r}\right)[\mu \mathrm{M}]$ was calculated as follows (Equation 2.3):

$\mathrm{C}_{\mathrm{r}}=\frac{\mathrm{C}_{\mathrm{b}}^{\mathrm{I}}}{\frac{\mathrm{F}_{\mathrm{m}+\mathrm{F}_{\mathrm{b}}^{\mathrm{I}}}^{\mathrm{I}}}{\mathrm{F}_{\mathrm{b}}^{\mathrm{I}}}}$

where $\mathrm{C}_{\mathrm{b}}{ }^{\mathrm{I}}$ is the benzene inflow concentration from a $22 \mathrm{mM}$ stock solution and $\mathrm{F}_{\mathrm{m}}^{\mathrm{I}}$ and $\mathrm{F}_{\mathrm{b}}^{\mathrm{I}}$ the flow rates of medium and the benzene stock solution in $\mathrm{ml}_{\text {day }}{ }^{-1}$, respectively.

The liquid dilution rate of the system $(F)$ was calculated according to Equation 2.4:

$\mathrm{F}=\frac{\mathrm{F}_{\mathrm{m}+\mathrm{F}_{\mathrm{b}}^{\mathrm{I}}}^{\mathrm{I}}}{\mathrm{V}}$

where $V$ is the total liquid volume in the culture vessel [L]. 


\section{Chapter 2.}

\section{$\underline{\text { Results }}$}

The biofilm culture continuously degraded benzene under nitrate-reducing conditions. At the beginning of biofilm formation at clear hydrophilic surfaces, biofilm was white after which it became brown over time (Figure S2.1).

\section{Continuous culture}

\section{$\underline{\text { Benzene degradation and kinetics }}$}

Benzene was stably degraded at a reservoir concentration $\left(C_{r}\right)$ of $105 \mu \mathrm{M}$ at four dilution rates from 0.25 to 2 day $^{-1}$. Up to dilution rates of 1 day $^{-1}$, benzene was degraded to below the detection limit of the GC method $(0.1 \mu \mathrm{M})$ with first-order benzene degradation constants increasing from 0.46-1.31 day ${ }^{-1}$ (Table 2.2).

Table 2.2 First-order benzene degradation rate constants (k) and half-lives (hours) at different liquid dilution rates of the continuous culture.

\begin{tabular}{ccc}
\hline Dilution rate $\left[\mathrm{day}^{-1}\right]$ & $\mathbf{k}\left[\mathbf{d a y}^{-1}\right]$ & Half-life [hours] \\
\hline 0.25 & 0.46 & 36.2 \\
0.50 & 0.62 & 26.8 \\
1 & 1.31 & 12.7 \\
2 & 3.04 & 5.5 \\
\hline
\end{tabular}

When the dilution rate was increased to 2 day $^{-1}$, the first-order benzene degradation constant was $3.04 \mathrm{day}^{-1}$, corresponding to a half-life of 5.5 hours (Table 2.2). However, the residual benzene concentration increased to $1.3 \mu \mathrm{M}$. Hence, the dilution rate of 1 day $^{-1}$ was used to assess the benzene degradation capacity of the continuous culture by stepwise increasing the reservoir benzene concentration from 105 to $715 \mu \mathrm{M}$ after equilibrium was reached between the liquid and headspace in the culture vessel (Figure 2.2). Between benzene inflow concentrations of $105 \mu \mathrm{M}$ up to $615 \mu \mathrm{M}$, benzene was degraded, albeit with steadily increasing residual benzene concentrations proportional to the increase in inflow concentrations. At a benzene reservoir concentration above $615 \mu \mathrm{M}$, a threshold value was reached after which the microbial community stopped degrading benzene. The experiment 
was stopped at a residual concentration of about $70 \mu \mathrm{M}$ because of the toxic effect of the accumulating benzene on the microbial community.

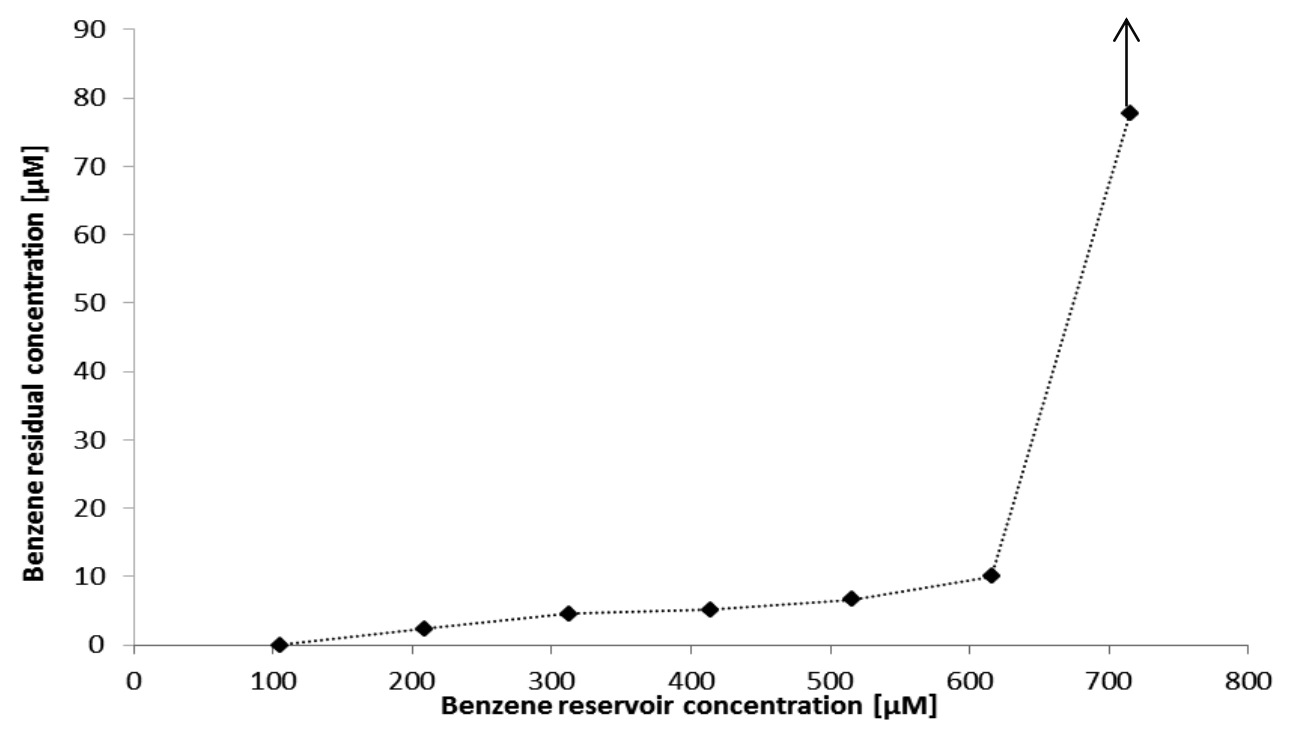

Figure 2.2 Residual benzene concentrations in the continuous biofilm culture after stepwise increased benzene reservoir concentrations at a liquid dilution rate of 1 day $^{-1}$. At a reservoir concentration of $615 \mu \mathrm{M}$ the benzene concentration continuously increased (indicated by the arrow at $715 \mu \mathrm{M}$ ) due to benzene toxicity on the microbial community.

At a dilution rate of 2 day $^{-1}$ the total protein content in the liquid (2 liter) and the white (642 $\mathrm{cm}^{2}$ ) and brown $\left(213 \mathrm{~cm}^{2}\right)$ biofilms in the culture vessel were $15 \mathrm{mg}, 233 \mathrm{mg}$ and $2485 \mathrm{mg}$, respectively, indicating that $99.5 \%$ of the microbial biomass in the culture vessel was present in the biofilms. The microbial biofilm and liquid community degraded benzene at a maximal rate of $0.15 \mu$ mol benzene.mg protein ${ }^{-1}$.day ${ }^{-1}$. To determine the possible diffusion limitation of benzene degradation, the stirring rate was varied between 100 and $200 \mathrm{rpm}$ in the culture vessel at benzene reservoir concentrations of $616,1385,1478$ and $1570 \mu \mathrm{M}$ and a dilution rate of $0.25 \mathrm{day}^{-1}$. During this period the residual benzene concentration remained below $2.5 \mu \mathrm{M}$ and did not increase at lower stirring rates of $100 \mathrm{rpm}$.

At a dilution rate of 0.25 day $^{-1}$, benzoic acid was detected at a concentration of $0.18 \mu \mathrm{M}$ in the liquid phase of the reactor, whereas the concentration of 4-hydroxy benzoic acid was 


\section{Chapter 2.}

below the detection limit of the LC-qTOF-MS $(<0.18 \mu \mathrm{M})$. No phenol was found in the reactor vessel.

After growth of the culture for 20 volume exchanges on a medium without vitamins and ammonium, the community still degraded $100 \mu \mathrm{M}$ benzene to below the detection limit (1 $\mu \mathrm{M})$.

Transfers of aggregates of biofilm from the continuous culture grew readily in batch cultures supplied with $10 \mathrm{mM}$ benzoate and $40 \mathrm{mM}$ nitrate. Microcosms inoculated with aggregates of biofilm and addition of $100 \mu \mathrm{M}$ benzene and $3.6 \mathrm{mM}$ nitrate degraded benzene at an average rate of $1.25 \pm 0.66 \mu \mathrm{M}$ benzene.day ${ }^{-1}$ with a degradation rate constant of 0.06 day $^{-1}$ that was about 50 times lower than that obtained in the continuous culture vessel at dilution rate 2 day $^{-1}$. The benzene degradation rate was about 700-fold lower than the maximal rate obtained in the continuous culture vessel at dilution rate 2 day $^{-1}$. Each time when benzene was depleted in the microcosm, benzene was replenished (Figure S2.2).

\section{Microbial community}

The biofilm and MiSeq sequencing characteristics for the different samples are described in Table 2.3. The phylogenetic diversity (PD whole tree) was similar for all samples with an average of $4.0 \pm 0.2$. The number of observed OTUs was higher for the liquid with white biofilm with an average of $139 \pm 3$ compared with an average of $109 \pm 1$ for the brown biofilm (Table 2.3). 
Table 2.3 Biofilm and MiSeq sample characteristics.

\begin{tabular}{|c|c|c|c|c|c|c|}
\hline \multirow{2}{*}{$\begin{array}{c}\text { Sample } \\
\text { name }\end{array}$} & \multicolumn{2}{|c|}{$\begin{array}{c}\text { Scraped off } \\
\text { biofilm area } \\
{\left[\mathrm{cm}^{2}\right]}\end{array}$} & \multirow{2}{*}{$\begin{array}{c}\text { Bacterial } \\
16 S \text { rRNA } \\
\text { genes/cm } \\
\text { biofilm }\end{array}$} & \multirow{2}{*}{$\begin{array}{c}\text { Sequence } \\
\text { reads per } \\
\text { sample }\end{array}$} & \multirow{2}{*}{ OTUs } & \multirow{2}{*}{$\begin{array}{c}\text { Phylogenetic } \\
\text { diversity }\end{array}$} \\
\hline & $\begin{array}{c}\text { White } \\
\text { biofilm }\end{array}$ & $\begin{array}{l}\text { Brown } \\
\text { biofilm }\end{array}$ & & & & \\
\hline Sample_1 ${ }^{\mathrm{a}}$ & 6.3 & 0 & $4.8 \pm 2.9 \times 10^{8}$ & $43410 \pm 33216$ & $140 \pm 3.2$ & $3.9 \pm 0.2$ \\
\hline Sample 2 & 6.3 & 0 & $4.2 \pm 2.0 \times 10^{8}$ & 52014 & 138 & 4.2 \\
\hline Sample_3 & 22.3 & 4 & $9.8 \pm 4.3 \times 10^{9}$ & 42427 & 108 & 3.8 \\
\hline Sample_4 & 22.3 & 8 & $13 \pm 5.7 \times 10^{9}$ & 53124 & 109 & 4.1 \\
\hline
\end{tabular}

${ }^{\text {a }}$ Average of three samples

${ }^{\mathrm{b}}$ Calculated based on liquid and biofilm protein (white biofilm $233 \mathrm{mg}$, brown biofilm $2485 \mathrm{mg}$ ) in the sample

Based on the MiSeq community analysis data, the relative abundance of Peptococcaceae in the samples containing brown biofilm was $8.8 \pm 0.0 \%$ and ranged from $28 \%$ to $49 \%$ in the liquid with white biofilm (Figure 2.3). MiSeq analysis also indicated that members of the Anaerolineaceae were relatively predominant in the two samples containing brown biofilm with $39 \%$ and $41 \%$ relative abundance in samples 3 and 4 , respectively (Figure 2.3). Other relatively predominant families in both the liquid with white biofilm and brown biofilm

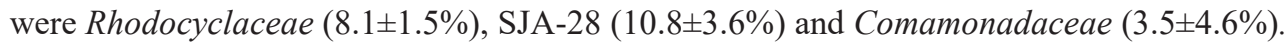




\section{Chapter 2.}

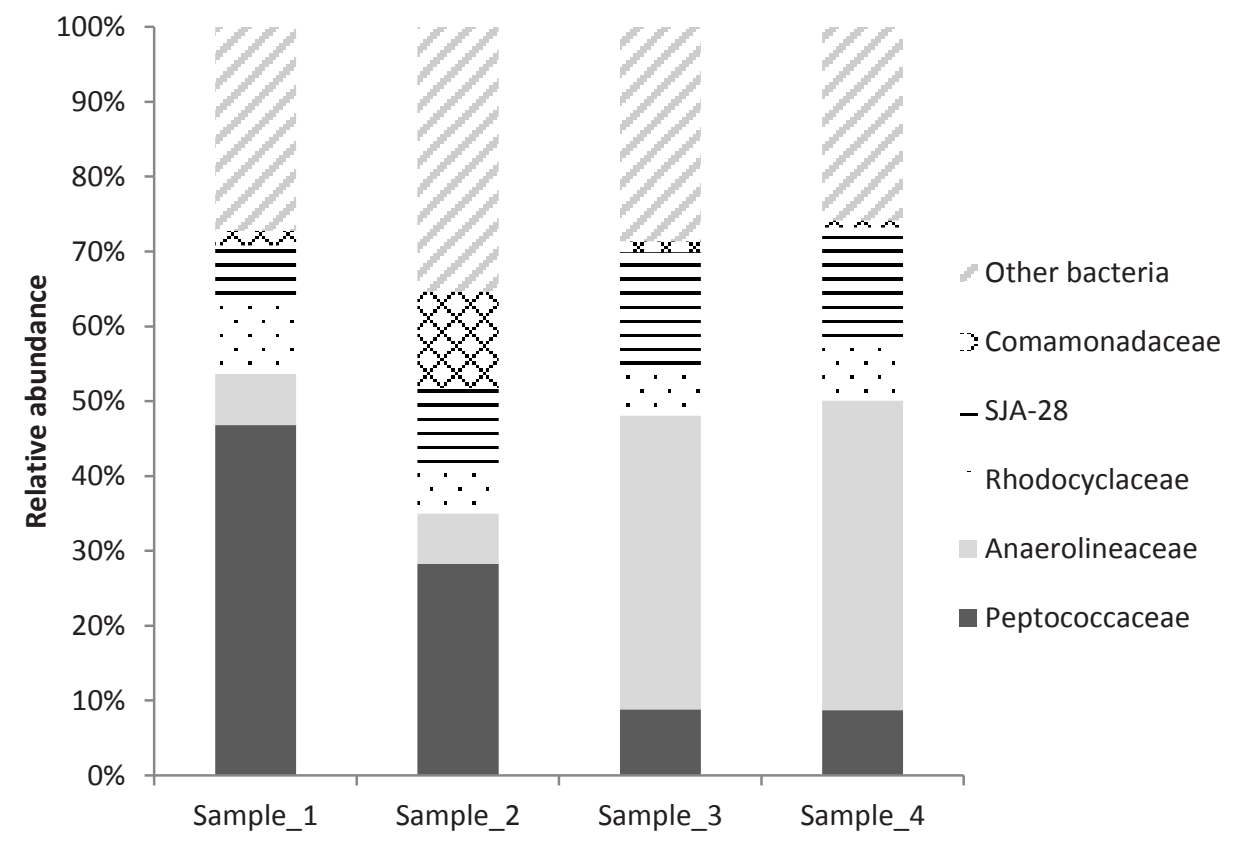

Figure 2.3 The relative abundances of partial bacterial 16S rRNA genes at family level in the continuous culture. Sample 1 and 2 contained 11\% biofilm and 89\% culture liquid protein. Sample_3 contained $70 \%$ biofilm and 30\% liquid protein biomass. Sample_4 contained 100\% biofilm. Only families with a relative abundance of $10 \%$ or higher in at least one of the biofilm samples are shown. Families occurring at lower relative abundances are shown together as "other bacteria".

Based on qPCR analysis, sample 4 contained $16 \pm 4.1 \times 10^{7}$ Peptococcaceae $16 \mathrm{~S}$ rRNA genes. $\mathrm{cm}^{-2}$ biofilm. The relative abundance of Peptococcaceae calculated using qPCR was similar to that obtained by MiSeq analyses (Figure S2.3).

The functional gene bss $A$ was not detected in the culture $(<3.1$ gene copies/ml sample). The other two functional genes studied here, $\operatorname{bam} A$ and $a b c A$, were detected in high copy numbers in all samples, i.e. $10 \pm 2.6 \times 10^{7}$ gene copies $/ \mathrm{ml}$ sample and $9.9 \pm 8.1 \times 10^{7}$ gene copies/ml sample, respectively (Figure 2.4). Peptococcaceae 16S rRNA gene copy numbers were generally about tenfold lower in the different samples than those of the $a b c A$ gene. 


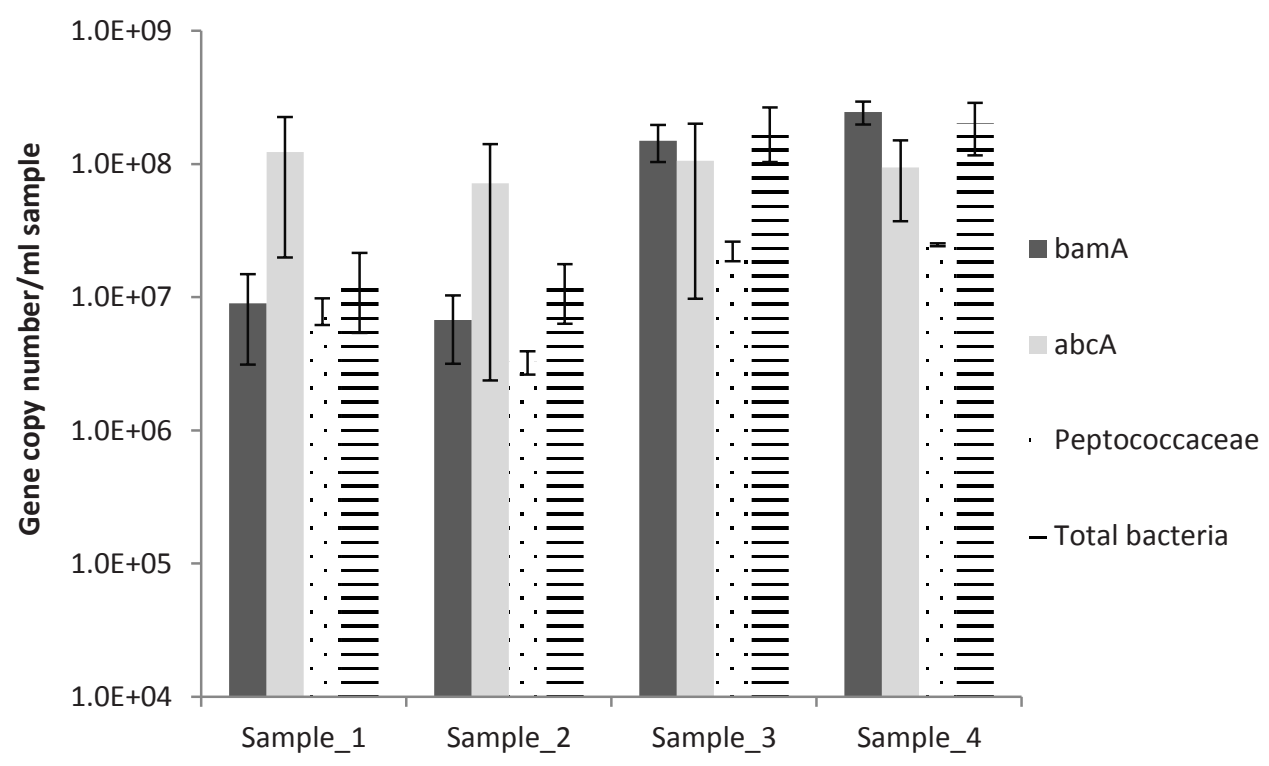

Figure 2.4 qPCR analyses of bamA, abcA and 16S rRNA genes of Peptococcaceae and total bacteria. Sample 1 and 2 contained $11 \%$ white biofilm corresponding to $4.8 \pm 2.9 \times 10^{8}$ and $4.2 \pm 2.0 \times 10^{8}$ bacterial $16 \mathrm{~S}$ rRNA genes $/ \mathrm{cm}^{2}$ biofilm, respectively. Sample_3 contained $70 \%$ biofilm corresponding to $9.8 \pm 4.3 \times 10^{9}$ bacterial $16 \mathrm{~S}$ rRNA genes $/ \mathrm{cm}^{2}$ biofilm. Sample_4 contained $100 \%$ biofilm corresponding to $13 \pm 5.7 \times 10^{9}$ bacterial $16 \mathrm{~S}$ rRNA genes $/ \mathrm{cm}^{2}$ biofilm. Error bars represent standard deviations based on analytical replicates of the same reactor sample.

\section{Continuous culture with retentostat}

In an attempt to further increase the activity of the benzene-degrading culture, a recirculation system with retentostat finger was added to retain biomass. Reservoir benzene concentrations were increased stepwise from $105 \mu \mathrm{M}$ to $1000 \mu \mathrm{M}$ to obtain the maximum degradation capacity of the culture with the retentostat finger at a dilution rate of 0.7 day $^{-1}$. Initially after the addition of the retentostat finger the first order benzene degradation rate constant was 3.93 day $^{-1}$ and the reservoir concentration at which the system collapsed and the microbial community was not able to degrade benzene due to toxicity was $715 \mu \mathrm{M}$ at a dilution rate of 0.7 day $^{-1}$ (Figure 2.5). After two subsequent replications of the retentostat experiment, the benzene degrading capacity of the culture decreased and the culture stopped degrading benzene at lower benzene reservoir concentrations of $566 \mu \mathrm{M}$ and 209 $\mu \mathrm{M}$, respectively (Figure 2.5 ). 


\section{Chapter 2.}

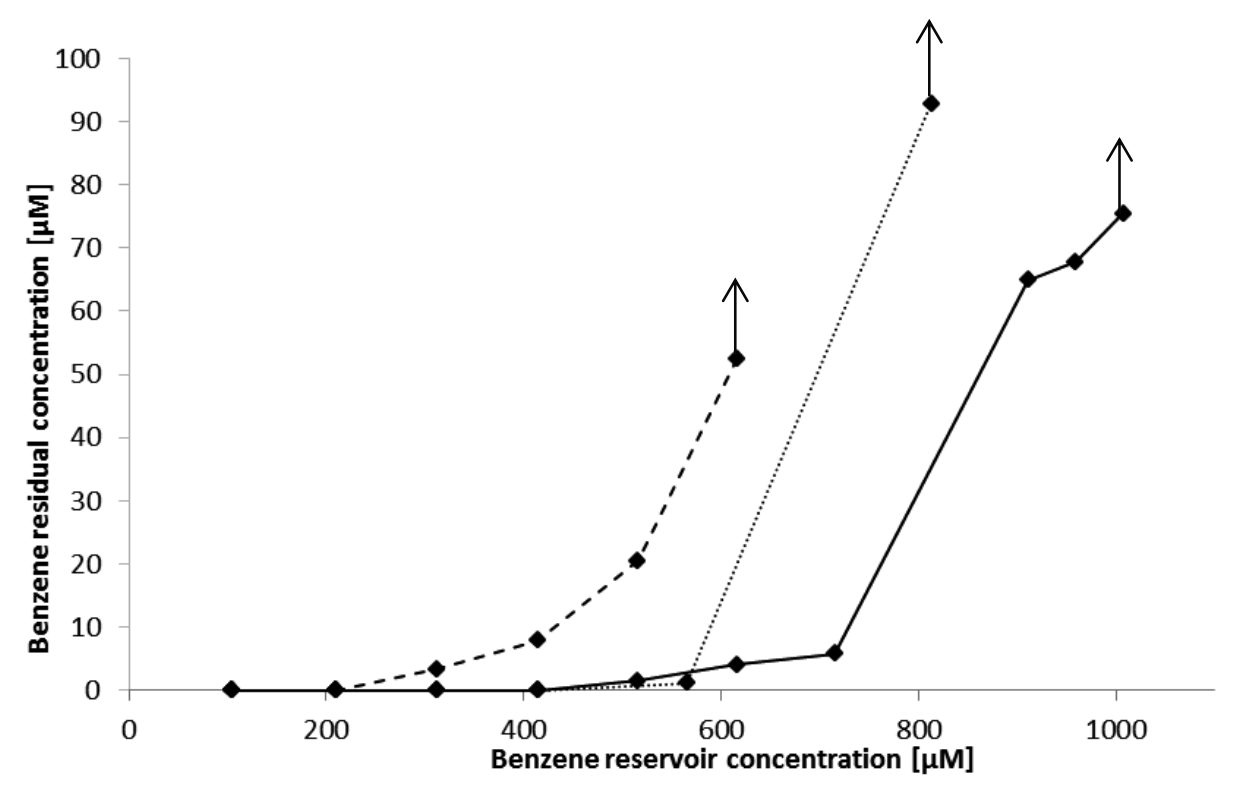

Figure 2.5 Benzene residual concentrations in the continuous culture with retentostat finger. The black line indicates the first (initial) experiment, the dotted line the second experiment at 88 days, and the dashed line the third experiment at 118 days after the initial experiment, respectively. The arrows show reservoir concentrations of 1007,840 and $616 \mu \mathrm{M}$, for the first, second and third experiments, respectively, where benzene degradation was continuously increased.

\section{Discussion}

The goals of this study were: (i) to determine the maximum anaerobic benzene degradation capacity of a continuous biofilm culture, (ii) to reveal the initial step in benzene degradation, (iii) to characterise the microbial composition of the liquid and biofilm-associated biomass at different ages using high throughput sequencing of PCR-amplified 16S rRNA gene fragments, and (iv) to obtain a highly active benzene degrading community. The benzene degradation rate constant $(\mathrm{k})$ of 3.04 day $^{-1}$ obtained in this study in the continuous culture vessel is about four times higher than the previously observed $0.70 \pm 0.12$ day $^{-1}$ that was obtained using the same microbial culture under denitrifying conditions [43]. To our knowledge, this study represents the highest anaerobic benzene degradation rate constant reported under denitrifying conditions (Table 2.4). 
Table 2.4 First order anaerobic benzene degradation rates under denitrifying conditions.

\begin{tabular}{|c|c|c|}
\hline Enrichment culture source & $\begin{array}{c}\text { Benzene } \\
\text { degradation } \\
\text { rate constant } \\
{\left[\text { day }^{-1}\right]}\end{array}$ & References \\
\hline $\begin{array}{l}\text { Benzene-degrading continuous biofilm culture in } \\
\text { reactor from a benzene polluted industrial location in } \\
\text { the Northern parts of the Netherlands } \\
\text { Benzene-degrading continuous biofilm culture from a } \\
\text { benzene polluted industrial location in the Northern }\end{array}$ & 3.04 & This study \\
\hline $\begin{array}{l}\text { parts of the Netherlands } \\
\text { Batch enrichment culture from an oil refinery in }\end{array}$ & $0.7 \pm 0.12$ & [43] \\
\hline Oklahoma & $0.29^{\mathrm{a}}$ & {$[60]$} \\
\hline $\begin{array}{l}\text { Benzene-degrading enrichment culture in microcosms } \\
\text { from an oil refinery in Oklahoma }\end{array}$ & $0.2^{\mathrm{a}}$ & {$[41]$} \\
\hline $\begin{array}{l}\text { Batch enrichment culture from a coal-tar contaminated } \\
\text { site in Glens Falls }\end{array}$ & $0.15^{\mathrm{a}}$ & {$[58]$} \\
\hline $\begin{array}{l}\text { Pure culture of Bacillus cereus from a gasoline } \\
\text { contaminated soil }\end{array}$ & $0.08^{\mathrm{a}}$ & {$[45]$} \\
\hline $\begin{array}{l}\text { Enrichment from gasoline contaminated groundwater } \\
\text { in microcosms }\end{array}$ & $0.05^{\mathrm{a}}$ & {$[44]$} \\
\hline
\end{tabular}

${ }^{\mathrm{a}}$ Calculated based on the benzene degradation in the discussed studies

Besides obtaining the benzene degradation rate constant in this study, a specific benzene degradation rate was calculated based on the amount of proteins in the reactor vessel. In previous studies aerobic degradation rates of 10 to $321 \mu$ mol benzene.mg dry weight ${ }^{-1}$.day ${ }^{-1}$ were reported ${ }^{108}$. Assuming an average protein content of 55\% (protein content of E. coli) of dry mass for bacteria [139], these rates correspond to 5.5 to $176.5 \mu \mathrm{mol}$ benzene.mg protein $^{-1}$.day ${ }^{-1}$. The anaerobic benzene degradation rate of $0.15 \mu \mathrm{mol}$ benzene.mg protein ${ }^{1}$.day $^{-1}$ found in the present study is therefore 37 to 1177 -fold lower than previously described aerobic degradation rates. In a previous study an anaerobic degradation rate of 4.9 to $9.3 \mu$ mol benzene.mg protein ${ }^{-1}$. day $^{-1}$ under denitrifying conditions was determined using enrichment cultures in microcosms from a gas station and a swamp in Canada [41]. These rates are 33 to 61-fold higher than the anaerobic benzene degradation rate found in the present study, which could be explained by the fact that more benzene degraders per protein were present and fewer organisms growing on other substrates like ammonium or vitamins supplied in our medium with concentrations of $68.2 \mathrm{mg} / \mathrm{l}$ and $2.6 \mathrm{mg} / \mathrm{l}$, respectively. Ac- 


\section{Chapter 2.}

cording to the growth yield of anammox bacteria ( $0.066 \mathrm{~mol}$ biomass carbon / mol ammonium) a maximum of $250 \mu \mathrm{M}$ carbon biomass could be produced with the supplied ammonium [140]. This corresponds to $42 \%$ of the carbon supplied as benzene. The amount of carbon supplied in vitamins corresponds to $124 \mu \mathrm{M}$, assuming an average molecular vitamin formula of $\mathrm{C}_{12} \mathrm{H}_{16} \mathrm{~N}_{2.6} \mathrm{O}_{3.4}$. This corresponds to $21 \%$ of the benzene carbon supplied. The bacterial community is able to degrade the benzene without the vitamins and ammonium in the medium, indicating that the supply of vitamins and ammonium in the medium is not required for benzene degradation. A higher amount of benzene degraders per protein in previous studies could be due to the fact that the microcosms were enriched through several serial transfers in the study of Ulrich et al. [41]. In addition, other explanations that could support this higher rate are: i) faster benzene-degrading organisms, ii) enrichment using a batch study corresponding to a high maximal growth and benzene degradation rate compared with limited substrate availability in a continuous culture used in this study corresponding to a lower maximal growth and benzene degradation rate. The rate of $0.15 \mu \mathrm{mol}$ benzene.mg protein ${ }^{-1}$. day ${ }^{-1}$ found in the present study at a dilution rate of 2 day $^{-1}$ may not be the maximal degradation rate for the residing microbial community, since this rate was measured under benzene limiting conditions. Experimental data indicated that benzene degradation was not limited through benzene diffusion from the bulk liquid into the biofilm $[141,142]$. This suggests that the maximal benzene degradation capacity obtained in this study was limited by the degradation kinetics of the microorganisms and/or the available biofilm surface in the culture. An increased benzene degrading capacity of the bioreactor is expected if more surface area for biofilm formation would be available. The benzene degradation rate decreased in this study when a liquid inoculum with biofilm aggregates from the reactor was used in a microcosm. The degradation rate in the microcosm was lower than that observed in the continuous culture since solely liquid with biofilm aggregates was used as inoculum instead of pure biofilm culture. Our results show that most of the benzene degraders reside in the biofilm since $99.5 \%$ of the microbial biomass was present in the biofilms as was suggested before [43].

The presence of benzoic acid $(0.18 \mu \mathrm{M})$ in the culture vessel, and absence of phenol suggests that benzene is degraded via an initial carboxylation [114]. Other observations that support this are i) the presence of the benzene carboxylase encoding $a b c A$ gene, and ii) the absence of $b s s A$ functional genes as determined by qPCR. The higher counts of the $a b c A$ 
gene compared with Peptococcaceae 16S rRNA gene copies may indicate that there are multiple $a b c A$ gene copies in the DNA of Peptococcaceae, that the primers are targeting similar genes, or that the $a b c A$ gene is also present in other organisms. While being beyond the scope of the current study, future experiments should include analysis of the putative benzene carboxylase gene at the RNA level to confirm that the $a b c A$ gene is expressed during growth on benzene.

A similar phylogenetic diversity was noted for all the liquid and biofilm samples, but lower numbers of OTUs in the brown biofilm indicate that the phylotypes observed in the brown biofilm were phylogenetically more distantly related than those present in the liquid phase and the white biofilm, as the diversity metric used here, PD whole tree, takes phylogenetic distance of community members into account [143]. The fact that benzoate degraders unable to degrade benzene were present may contribute to the high bacterial diversity within the biofilm reactor. A rapid and extensive growth was indeed observed in transfers of the continuous culture in microcosms with benzoate.

Relatively high numbers of Anaerolineaceae 16S rRNA genes were detected in the biofilms. Currently, a few members of the family Anaerolineaceae are described and isolated that are strict anaerobes able to grow on oil components and often found under sulphate-reducing or methanogenic conditions [144-146]. Anaerolineaceae family members grow by fermentation, generating fermentation intermediates such as acetate and hydrogen from alkanes, which can be oxidized by other community members $[144,146]$. Further compiling evidence points towards potential roles of Anaerolineaceae in the degradation of aromatic compounds. For example, uncultured Anaerolinea spp. have been shown to scavenge organic compounds from lysed cells [147]. Microbial community analysis of an Anaerobic Sequencing Batch Reactor (ASBR) revealed members of the family Anaerolineaceae as putative anaerobic phenol degraders [148]. Kümmel et al. (2015) also obtained enrichments growing on polycyclic aromatic hydrocarbons (PAHs), benzene, toluene, ethylbenzene and xylene (BTEX), highly enriched in Anaerolineaceae [149]. It is possible that Anaerolineaceae are primary benzene degraders in the biofilm or that they are enriched within the biofilm because they oxidize benzoate excreted by primary benzene carboxylating Peptococcaceae [43]. Less than $0.5 \%$ of the benzene supplied to the reactor was detected as benzoate. Peptococcaceae may excrete benzoate from leaky cells or benzoate might be produced at the membrane as suggested for Dechloromonas strain RCB [150]. Therefore it is 


\section{Chapter 2.}

reasonable to assume that Peptococcaceae consume a major fraction of the benzene. It has previously been suggested that Peptococcaceae may be responsible for the first step in benzene degradation and Azoarcus scavenges electrons from benzoate, produced by leaky of lysed cells [114].

Being another predominant family observed in our study, members of the Comamonadaceae have been shown to be able to degrade phenol and toluene under denitrifying conditions [151, 152]. Recently, a Comamonas sp. was isolated from our reactor, which was not able to grow on benzene but grew on phenol, p-hydroxybenzoate and methylhydroxybenzoate with nitrate (Balk, personal communication). Possibly also other metabolites such as phenol are produced in the continuous culture. Members of the family SJA-28 are affiliated to the phylum Ignavibacteria. Current knowledge about Ignavibacteriaceae is limited. Kümmel et al. (2015) observed an increase in Ignavibacteriaceae in an enrichment culture obtained from a sediment sample contaminated with PAHs and BTEX. Besides SJA-28, Rhodocyclaceae were also relatively predominant. It was previously suggested that Rhodocyclaceae are involved in nitrate reduction and syntrophically associated with the anaerobic benzene degrading Peptococcaceae [43, 114]. The recently isolated Pseudomonas stutzeri strain $\mathrm{BN}$ and a bacterium belonging to the Bacillus sp. from our reactor were not detected in the samples based on community analysis. It is possible that these strains are minor degrading populations, but based on the low relative abundance not responsible for the large amount of benzene degradation. The recently isolated Ochrobactrum sp. was present in our community data.

After the addition of the retentostat finger to further increase the activity of the benzene degrading community, the degradation rate constant was initially higher than without retentostat. However, replication of the experiment with the retentostat finger resulted in a decreased benzene degradation capacity. This decreased degradation capacity may be explained by the selection of microbes with a high affinity and low maximum consumption rate for benzene. During retention of biomass more microbial cells in the culture vessel are expected to share the same amount of substrate. This induces starvation, and physiological adaptation of the benzene degrading microorganisms or adaptation of the community composition to low substrate concentrations, generally resulting in a reduced maximum degradation rate $[120,153,154]$. At the same time a selective pressure is imposed, favoring the enrichment of microorganisms with high affinity for benzene, which may be expected to 
have relatively low maximal growth and degradation rates [155]. When the continuous culture was restored after the retentostat experiments, it degraded benzene to below $0.1 \mu \mathrm{M}$ up to dilution rates of $4 \mathrm{day}^{-1}$ at a benzene reservoir concentration of $105 \mu \mathrm{M}$. This indicates that the diverse microbial community in the biofilm readily adjusts to changing conditions and was able to restore its function after a system perturbation, indicating resilience at least across the varying conditions applied in this study.

This study suggests that other bacteria besides Peptococcaceae have an important role in the anaerobic benzene degradation. Our data suggest that benzene is degraded through an initial carboxylation. This study also represents the highest anaerobic benzene degradation rate constant published so far and confirms that the microbial community in biofilms can be important for benzene degradation under conditions of continuous supply. It is therefore important to further study the significance of biofilms during natural attenuation at contaminated sites. This may also broaden our options to use biofilms for stimulated bioremediation through biostimulation and bioaugmentation at contaminated groundwater systems. 
Chapter 2.

\section{Supplementary Figures and Tables}

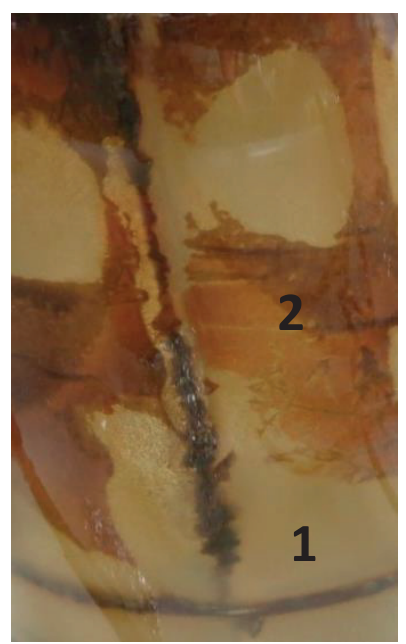

Figure S2.1 White (1) and brown (2) biofilm attached at the glass wall and metal surfaces in the continuous culture.

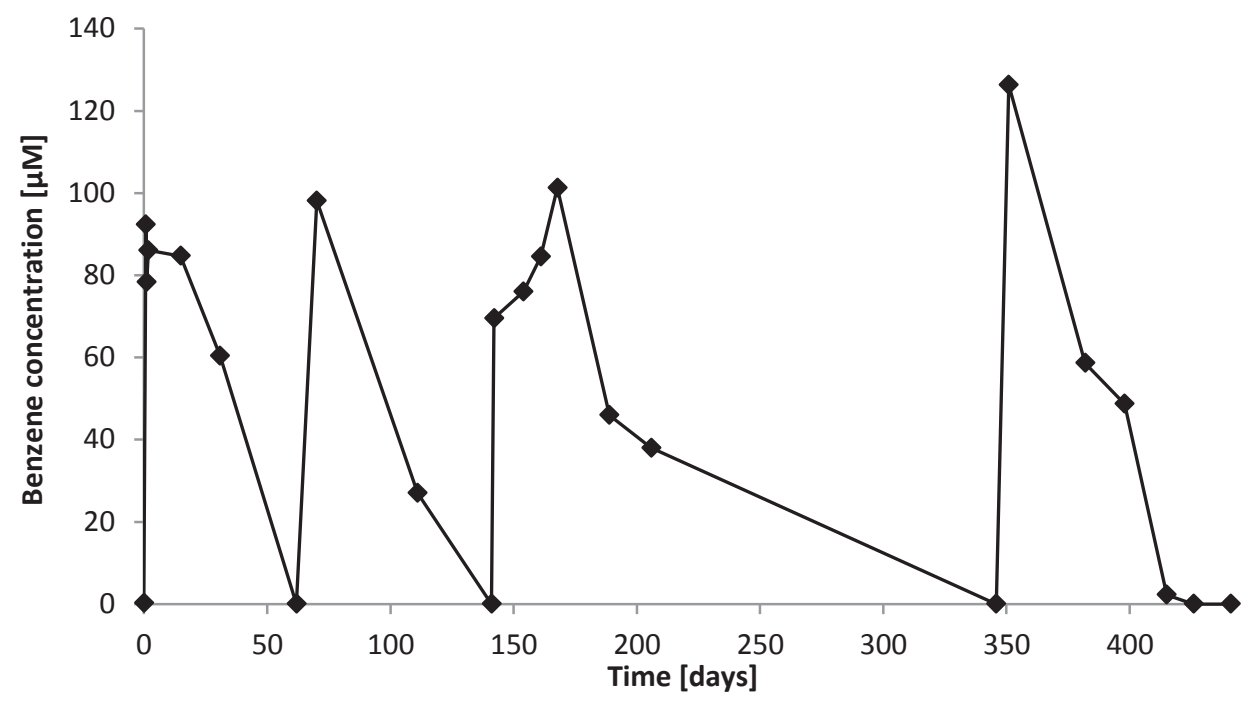

Figure S2.2 Time course analysis of benzene concentration in the microcosm with transferred liquid from the continuous culture supplied with $100 \mu \mathrm{M}$ benzene and $3.6 \mathrm{mM}$ nitrate. After each benzene depletion, benzene was replenished. 


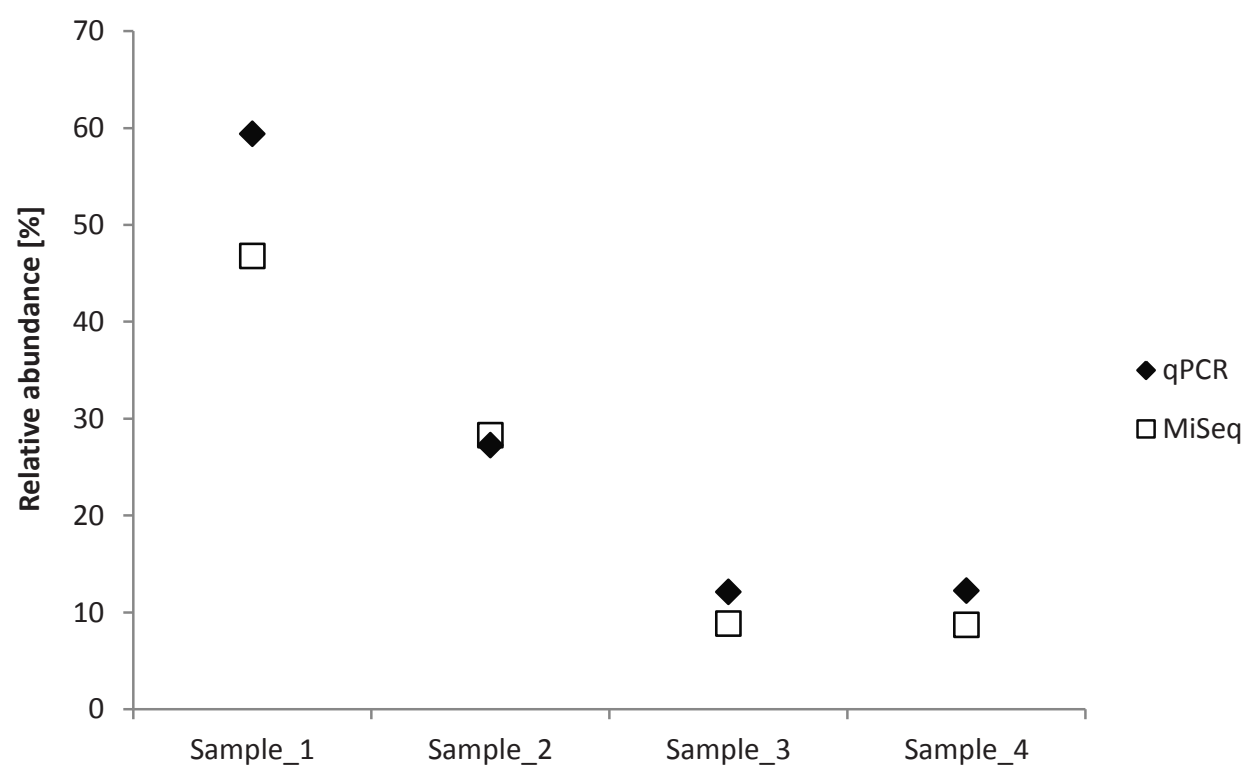

Figure S2.3 Relative abundance of Peptococcaceae versus total bacterial 16S rRNA gene copies in the different samples, calculated using qPCR (closed diamonds) and MiSeq (open squares). 
Chapter 2.

54 


\section{Chapter 3. Anaerobic degradation of a mixture of MtBE,}

\section{EtBE, TBA and benzene under different redox conditions}

Marcelle J. van der Waals, Charles Pijls, Anja J. C. Sinke, Alette A. M. Langenhoff, Hauke Smidt and Jan Gerritse

This chapter has been published as:

Anaerobic degradation of a mixture of MtBE, EtBE, TBA and benzene under different redox conditions, Applied Microbiology and Biotechnology, 2018 102:7 p. 3387-3397 Marcelle J. van der Waals, Charles Pijls, Anja J. C. Sinke, Alette A. M. Langenhoff, Hauke Smidt and Jan Gerritse (2018) 


\section{Chapter 3.}

\section{Abstract}

The increasing use of biobased fuels and fuel additives can potentially change the typical fuel related contamination in soil and groundwater. Anaerobic biotransformation of the biofuel additive ethyl tert-butyl ether (EtBE), as well as of methyl tert-butyl ether (MtBE), benzene and tert-butyl alcohol (TBA, a possible oxygenate metabolite), was studied at an industrially contaminated site and in the laboratory. Analysis of groundwater samples indicated that in the field MtBE was degraded, yielding TBA as major product. In batch microcosms, MtBE was degraded under different conditions: unamended control, with medium without added electron acceptors, or with ferrihydrite or sulphate (with or without medium) as electron acceptor, respectively. Degradation of EtBE was not observed under any of these conditions tested. TBA was partially depleted in parallel with MtBE. Results of microcosm experiments with MtBE substrate analogues, i.e. syringate, vanillate or ferulate, were in line with the hypothesis that the observed TBA degradation is a cometabolic process. Microcosms with ferulate, syringate, isopropanol or diethyl ether showed EtBE depletion up to $86.5 \%$ of the initial concentration after 83 days. Benzene was degraded in the unamended controls, with medium without added electron acceptors, and with ferrihydrite, sulphate, or chlorate as electron acceptor, respectively. In the presence of nitrate, benzene was only degraded after addition of an anaerobic benzene degrading community. Nitrate and chlorate hindered MtBE, EtBE and TBA degradation.

\section{Introduction}

The use of renewable resources, such as bioethanol, as basis for automotive fuels is stimulated under the European Biofuels Directive (EC 2003) to reduce greenhouse gas emissions. Both fossil-based and biobased fuels may enter the groundwater through incidents and spillages. An overview compiled by Concawe (division of the European Petroleum Refiners Association) showed that oxygenates are present in groundwater, drinking water, surface water, runoff water, precipitation and air in the European environment [10]. The oxygenate methyl tert-butyl ether (MtBE) is a synthetic volatile organic compound added to gasoline to increase its octane number, i.e. performance. Due to its high water solubility, low odour threshold, and health concerns, MtBE contamination is a widespread problem that requires remediation $[13,14]$. Although the tertiary carbon structure and stable ether bond suggest that the MtBE molecule will not be readily degraded by microorganisms under anaerobic conditions, degradation has been observed under a variety of redox conditions, including 
methanogenic, nitrate-reducing, manganese-reducing, iron-reducing, and sulphate-reducing conditions [19, 23, 26, 28-30, 32, 34, 156-160]. Key metabolites of aerobic MtBE degradation are tert-butyl alcohol (TBA), tert-butyl formate (TBF) and 2-hydroxy isobutyric acid (HIBA) [161]. The detection of TBA indicates that ether bond cleavage is the initial step in anaerobic MtBE degradation. It has been found that under anoxic conditions TBA is often a recalcitrant product of MtBE degradation [16]. However, mineralization of MtBE without accumulation of TBA has been shown under denitrifying and oxic conditions [31].

In the past years, MtBE has been partially replaced by ethyl tert-butyl ether (EtBE), a biofuel oxygenate synthesized from (bio)ethanol and isobutylene. Due to its high solubility and persistence, EtBE disperses rapidly in the environment [12]. Little is known about anaerobic EtBE degradation. Several studies have reported lack of any anaerobic degradation of EtBE [20, 34, 162]. In contrast, Yeh and Novak demonstrated EtBE degradation in soils under denitrifying and methanogenic conditions [21]. Compound-specific stable isotope analysis (CSIA) revealed insignificant carbon isotope fractionation, and low hydrogen isotope fractionation up to $+14 \%$ along an anoxic EtBE plume at a contaminated field site in the Central West of Spain suggesting anaerobic biodegradation of EtBE [22].

In addition to TBA being produced as metabolite during MtBE and EtBE degradation, it is also frequently used as a polar organic solvent in flavours, perfumes or paint removers. TBA is highly soluble in water and is considered as a widespread contamination problem [14]. Several studies in the absence or presence of MtBE have concluded that TBA is either degraded with rates ranging between $<0.14$ and $2 \mu \mathrm{M} /$ day $/ \mathrm{g}$ dry soil, or persists in anoxic groundwater $[20,21,33,34]$. Under methanogenic conditions no TBA degradation has been observed $[34,37]$. TBA consumption in anoxic microcosms with iron(III) as electron acceptor has been demonstrated in microcosms [32]. A direct link between TBA oxidation and electron acceptor reduction has, however, not been demonstrated. Aerobically, TBA can be degraded via 2-methyl-2-hydroxy-1-propanol, HIBA, 2-propanol, acetone and hydroxyacetone to carbon dioxide and water.

Benzene is a natural constituent of crude oil, has a high octane number, and is commonly present in (bio)fuel blends [163]. Massive production and use of benzene combined with its high mobility, relatively low anaerobic biodegradation rates and carcinogenicity make benzene one of the most widespread groundwater contaminants of concern. Therefore, benzene is typically considered as risk determining compound in fuels. Anaero- 


\section{Chapter 3.}

bic benzene biodegradation has been described under denitrifying, iron-reducing, sulphatereducing and methanogenic conditions [164].

Several studies have investigated the effects of mixtures of fossil-based and biobased fuels on degradation of the single components. Both antagonistic and synergistic effects have been reported during degradation of mixtures of benzene, toluene, ethylbenzene, xylene (BTEX) and MtBE. The presence of BTEX was shown to enhance aerobic MtBE degradation by a mixed microbial culture under continuous flow conditions [165]. On the contrary, biodegradation of MtBE in the subsurface was inhibited largely by the presence of ethylbenzenes and xylenes and partially inhibited by benzene and toluene until MtBE had migrated beyond the BTEX plume [166]. The preferential utilization of ethanol under aerobic, denitrifying, iron-reducing, sulphate-reducing and methanogenic conditions resulted in a negative effect on BTEX degradation [87, 167, 168]. Bioaugmentation with a benzene enriched methanogenic consortium enhanced anaerobic benzene degradation in a mixture of BTEX and ethanol [99].

The concurrence of MtBE, EtBE, TBA and benzene may affect the microbial degradation potential for both fossil-based and biobased components. Nevertheless, to our knowledge, studies on the anaerobic degradation of a mixture of MtBE, EtBE, TBA and benzene have not been reported. In this study anoxic groundwater from a contaminated location was used to determine the microbial degradation potential for a mixture of benzene, MtBE, EtBE, and TBA, the latter being a degradation product of MtBE and EtBE.

The aims of the present study are therefore to (i) determine the potential of an indigenous microbial community from a contaminated site for the anaerobic degradation of MtBE, EtBE, TBA and benzene in a mixture, (ii) determine the effect of electron acceptors nitrate, sulphate, amorphous 2-line ferrihydrite and chlorate on the anaerobic degradation, and (iii) determine anaerobic TBA degradation and stimulate this process using MtBE and EtBE substrate analogues and potential metabolites as substrates. 


\section{Materials and Methods}

\section{Field site, modelling and sampling procedures}

Experiments were focused on MtBE, EtBE, TBA and benzene contaminated groundwater at an industrial site. At this site the redox conditions varied from iron reducing to methanogenic, and the $\mathrm{pH}$ was around 7 (Table S3.1). At the impacted area, the contamination was located between 9 and 20 metres depth below the surface in an anoxic aquifer. Two source zones of MtBE were present with a maximum MtBE concentration of about $4000 \mu \mathrm{M}$. The highest concentrations of EtBE, TBA and benzene detected in 2009 were $1 \mu \mathrm{M}(88 \mu \mathrm{g} / \mathrm{l})$, $130 \mu \mathrm{M}(9636 \mu \mathrm{g} / \mathrm{l})$ and $260 \mu \mathrm{M}(20309 \mu \mathrm{g} / \mathrm{l})$, respectively. The average MtBE, TBA and benzene concentrations per layer in 2009, 2013 and 2015 are given in Table S3.2. The data for MtBE, TBA and benzene depletion at the field site were compared with an existing hydrological model and contaminant fate and transport model that was calibrated using field data on measured heads, porosity, organic matter content and long term diver data on groundwater fluctuations [169, 170].

Groundwater was collected from a monitoring well located in one of the MtBE source zones, in gas tight bottles of 1 litre. The bottles were filled completely with groundwater to prevent the introduction of oxygen, were transported in a cool box with ice the same day to the laboratory, and were stored at $4{ }^{\circ} \mathrm{C}$ for preparation of microcosms within 14 days after sampling.

\section{Microcosm incubations}

Microcosms were prepared under a $80 / 20 \% \mathrm{~N}_{2} / \mathrm{CO}_{2}$ (v/v) flow in $250 \mathrm{ml}$ serum bottles (Glasgerätebau Ochs $\mathrm{GmbH}$, Bovenden, Germany) and crimp-sealed with viton rubber stoppers (Rubber BV, Hilversum, the Netherlands) and aluminium crimp caps (Grace, Maryland, USA). Traces of $\mathrm{O}_{2}$ were stripped from the $\mathrm{N}_{2} / \mathrm{CO}_{2}$ gas as described previously [57]. The microcosms contained $90 \mathrm{ml}$ of groundwater contaminated with MtBE, TBA and benzene, and amended with $50 \mu \mathrm{M}$ EtBE added from a $20 \mathrm{mM}$ anoxic, autoclaved stock solution. One series of microcosms with different electron acceptors was amended with 10 $\mathrm{ml}$ of 10-fold concentrated anoxic, sterile mineral medium with vitamins that was prepared as described previously, but without the addition of nitrate [57]. No medium was added to a second series of microcosms with the addition of only electron acceptors. Electron acceptors, i.e. nitrate $\left(\mathrm{NaNO}_{3}\right)$, chlorate $\left(\mathrm{NaClO}_{3}\right)$, iron (amorphous 2-line ferrihydrite) [171] or 


\section{Chapter 3.}

sulphate $\left(\mathrm{Na}_{2} \mathrm{SO}_{4}\right)$, were added from separately autoclaved stock solutions of $70.5 \mathrm{mM}$ to final concentrations of $2.4 \mathrm{mM}$. The $\mathrm{pH}$ of the prepared medium was 7.0. The microcosms were incubated upside down and continuously shaken at $100 \mathrm{rpm}$ (Certomat, B|Braun, Melsungen, Germany) in the dark at $20^{\circ} \mathrm{C}$. Abiotic control microcosms were autoclaved and contained $100 \mathrm{mg} / 1 \mathrm{HgCl}_{2}$ and $2 \mathrm{mg} / \mathrm{NaN}_{3}$. Unamended control microcosms, not receiving any medium and/or electron acceptors, were used as representatives for the natural condition. When MtBE or benzene was depleted, the compound was added to approximately $100 \mu \mathrm{M}$ using diluted anoxic, autoclaved $20 \mathrm{mM}$ stock solutions.

\section{TBA cometabolism experiment}

Serum vials (10 ml, Grace) with an $80 / 20 \% \mathrm{~N}_{2} / \mathrm{CO}_{2}(\mathrm{v} / \mathrm{v})$ headspace, crimp sealed with a viton rubber stopper and aluminium crimp cap were prepared with medium and $5 \mathrm{mM}$ methanol (Merck, Darmstadt, Germany), isopropanol (Sigma-Aldrich, Missouri, USA), diethyl ether (Sigma-Aldrich), syringic acid (Fluka analytical, St. Louis, USA), ferulic acid (Sigma-Aldrich) or vanillic acid (Boom, Meppel, the Netherlands) neutralized with $1 \mathrm{M}$ $\mathrm{NaOH}$ stock solution, respectively. These substrates were selected, since they are potential metabolites of MtBE and TBA degradation (methanol and isopropanol, respectively), or because they may be EtBE and MtBE analogues containing ethoxy (diethyl ether) or methoxy groups (syringic acid, ferulic acid and vanillic acid), respectively. Serum vials were filled with $2.4 \mathrm{ml}$ liquid from an unamended control or medium microcosm, respectively. The TBA concentration in the vials was regularly measured using solid phase microextraction (SPME) on a gas chromatograph with flame ionization detector (GC-FID) as described below. Concentrations of methanol, ethanol, isopropanol and diethyl ether in the serum vials were also regularly measured using a GC-FID equipped with an SPME fiber.

TBA (Sigma-Aldrich), MtBE (Rathburn Chemicals, Walkerburn, Scotland), benzene (Janssen Chimica, Beerse, Belgium) and EtBE (Sigma-Aldrich) were of a purity of $>99 \%$.

\section{Analytical procedures}

Headspace samples of $0.5 \mathrm{ml}$ were taken from the microcosms to measure methane using a $1 \mathrm{ml}$ sterile syringe (B|Braun) and $0.5 \times 25 \mathrm{~mm}$ needle (Henke Sass Wolf, Tuttlingen, Germany) and measured on a Varian 3800 GC-FID as previously described [57]. Liquid samples of $0.3 \mathrm{ml}$ for measuring MtBE, EtBE, TBA and benzene were taken from the microcosms using a $1 \mathrm{ml}$ sterile syringe (B|Braun) and 0.5x16 mm needle (Henke Sass Wolf) and 
Anaerobic degradation of a mixture of MtBE, EtBE, TBA and benzene injected in a $10 \mathrm{ml}$ headspace vial (Grace) containing $7 \mathrm{ml}$ of $20 \mathrm{mg} / 1$ mercury chloride and $2 \mathrm{ml}$ of $0.93 \mu \mathrm{g} / \mathrm{ml} 1$-propanol as internal standard (Acros, Geel, Belgium). SPME was performed on these aqueous solutions using a $75 \mu \mathrm{m}$ CAR/PDMS 24ga autosampler fiber, which was conditioned according to the manufacturer's protocol (Sigma-Aldrich). Six standards from 10-200 $\mu \mathrm{M}$ in serum bottles crimp sealed with viton stoppers and aluminium caps were used for calibration. An extraction time of 30 minutes and a desorption time of 3 minutes were found as optimum (Figure S3.1). A Combi-PAL autosampler (CTC analytics, Zwingen, Switzerland) with SPME fiber holder and agitator was used for this GC method. Each measurement sequence contained a calibration line and quality controls (MilliQ and calibration sample) within the sequence because of degrading fiber adsorption potential due to high benzene concentrations [172]. The coefficient of variation of this method was $5 \%$. The potential metabolites detected with this method include: methanol, TBF, ethanol, TBA, acetone, isopropanol and N-propanol.

Ion analyses were done using $0.1 \mathrm{ml}$ supernatant of shortly ( 5 seconds) centrifuged samples (5000 x g) (Eppendorf centrifuge 5424, Hamburg, Germany) diluted up to $1 \mathrm{ml}$ with MilliQ water and measured on a Dionex ICS-1500 equipped with an Ionpac AS19 anion-exchange column and an AERS 500 suppressor (Dionex Corp., Sunnyvale, CA). The potassium hydroxide gradient to separate anions was as follows: $10 \mathrm{mM}$ from 0 to 10 minutes and 10 to $45 \mathrm{mM}$ from 10 to 25 minutes with a flow rate of $1 \mathrm{ml} / \mathrm{min}$. The sample injection volume was $25 \mu 1$.

Compound Stable Isotope Analysis (CSIA) was performed in 2009 (Supplementary information) and 2013 by Hydroisotop (www.hydroisotop.de) using isotope ratio mass spectrometry (GC-IRMS) related to the Vienna PDB standard. Groundwater samples were fixed in the field with a pellet sodium hydroxide in $100 \mathrm{ml}$ serum bottles.

\section{DNA extraction and molecular analyses}

Biomass was concentrated from $10 \mathrm{ml}$ microcosm samples by vacuum filtration on $0.2 \mu \mathrm{m}$ pore-size filters (Merck Millipore). Filters were crushed with a sterilized wooden tooth pick and total DNA was extracted using the MoBio Powerlyzer DNA isolation kit (MoBio, CA, USA). DNA was stored at $-80^{\circ} \mathrm{C}$ until further molecular analyses. The total bacterial $16 \mathrm{~S}$ rRNA gene and the $a b c A$ gene encoding benzene carboxylase were quantified as described previously [57]. For this study a quantitative real-time PCR assay was designed using Pri- 


\section{Chapter 3.}

mer3 software $[134,135]$ to detect genes coding for isobutyryl-CoA mutase (icmA) that has been shown to be a key enzyme involved in MtBE degradation in Aquincola tertiaricarbonis [173]. The designed primer pair (F695) 5'-ACATCTCGGGCTACCACATC-3' (R868) 5'-CCTCGAAGAAGTCACCTTGC-3' with TaqMan probe 5'CTGGCCAACCTGATCACCTACGT-3' (757) was evaluated in silico using the publicly accessible NCBI BLAST search tool [136]. The icmA gene assay was performed in $25 \mu 1$ volumes as described elsewhere [57]. The temperature program on an IQ5 Icycler (Bio-rad, Veenendaal, the Netherlands) was: 5 minutes at $95^{\circ} \mathrm{C}$ followed by 45 cycles of 20 seconds at $95^{\circ} \mathrm{C}, 60$ seconds at $58^{\circ} \mathrm{C}$ and a final elongation step of 3 minutes at $72^{\circ} \mathrm{C}$. Normalized $a b c A$ and $i c m A$ gene counts were calculated as a concentration relative to the bacterial $16 \mathrm{~S}$ rRNA gene count.

\section{First order degradation rate constant calculation}

First-order degradation rate constants $(\mathrm{k})$ were calculated according to Equation 3.1 [111].

$\mathrm{k}=\left(\frac{\log \left(\mathrm{x}_{1}\right)-\log \left(\mathrm{x}_{2}\right)}{\mathrm{t}_{2}-\mathrm{t}_{1}}\right)$

where $t_{2}-t_{1}$ is the time interval in days; $x_{1}$ is the concentration at time $t_{1}$ and $x_{2}$ is the concentration at time $t_{2}$.

\section{$\underline{\text { Results }}$}

\section{Field data}

In 2015 MtBE concentrations had decreased strongly compared to measurements in 2009 and 2013. Based on the average concentration in all measured monitoring wells, only $2 \%$ of the MtBE was left in 2015 (Table S3.2). Benzene concentrations had decreased in the shallow layers of the aquifer up to $25 \mathrm{~m}$ below surface in 2015 as compared with 2013. MtBE, TBA and benzene concentrations did not change in the deeper layer of 25-30 m below surface from 2009 till 2015. In 2009, the $\delta^{13} \mathrm{C}$ for MtBE ranged from -24 to $-31 \%$. In 2013, MtBE $\delta^{13} \mathrm{C}$ had increased up to $+50 \%$. The TBA $\delta^{13} \mathrm{C}$ was constant ranging from -27 to $-31 \%$ over 2009-2013. Overall the fate and transport model achieved a fairly good correlation with long term benzene and MtBE field monitoring data at a Normalised Root Mean Square Deviation (NRMSD) of about 14\%. Although the limited dataset of TBA demonstrated a NRMSD of about $14 \%$, the overall observation was that correlation of TBA field data was 
unsatisfactory. In order to fit the TBA plume, complete lack of degradation was assumed. First order degradation rate constants for benzene and MtBE of $0.0007 \mathrm{day}^{-1}$ in the model were representative for the field plume situation.

In field samples from 2010, an average $16 \mathrm{~S}$ rRNA total bacterial gene count of $5.0 \pm 8.5 \times 10^{5}$ gene copies/ml sample was detected. The functional gene, icmA, was detected in 3 out of 14 groundwater monitoring wells. Normalized gene counts were relatively low, ranging from $1.5 \times 10^{-5}$ to $2.7 \times 10^{-4}$. The functional gene, $a b c A$, was detected in 2009,2013 and 2015 at stable concentrations with an average concentration of $1.2 \pm 2.4 \times 10^{-4}$ relative to the total bacterial 16S rRNA gene copies.

\section{Biodegradation of MtBE, EtBE, TBA and benzene in microcosms}

In microcosms with groundwater, MtBE was degraded in the unamended control and with the addition of medium and ferrihydrite, at first order rate constants of $0.02 \pm 0.002$ day $^{-1}$, 0.07 day $^{-1}$ and 0.04 day $^{-1}$, respectively (Table 3.1). Under sulphate reducing conditions with the addition of medium, the MtBE first order degradation rate constant of 0.06 day $^{-1}$ was higher than without the addition of medium $\left(0.03\right.$ day $\left.^{-1}\right)$ (Table 3.1). The concentration data for benzene, MtBE and electron acceptors were used to calculate electron balances. Under ferrihydrite reducing conditions, more electrons were released from benzene and MtBE (assuming complete oxidation to $\mathrm{CO}_{2}$ ) than the electrons used to reduce iron(III) to iron(II) with no detectable net production of metabolites (Table S3.3). Under sulphate reducing conditions fewer electrons were released from benzene and MtBE than the electrons used to reduce sulphate to sulphide (Table S3.3). In sterile control microcosms no degradation was observed. 
Chapter 3.

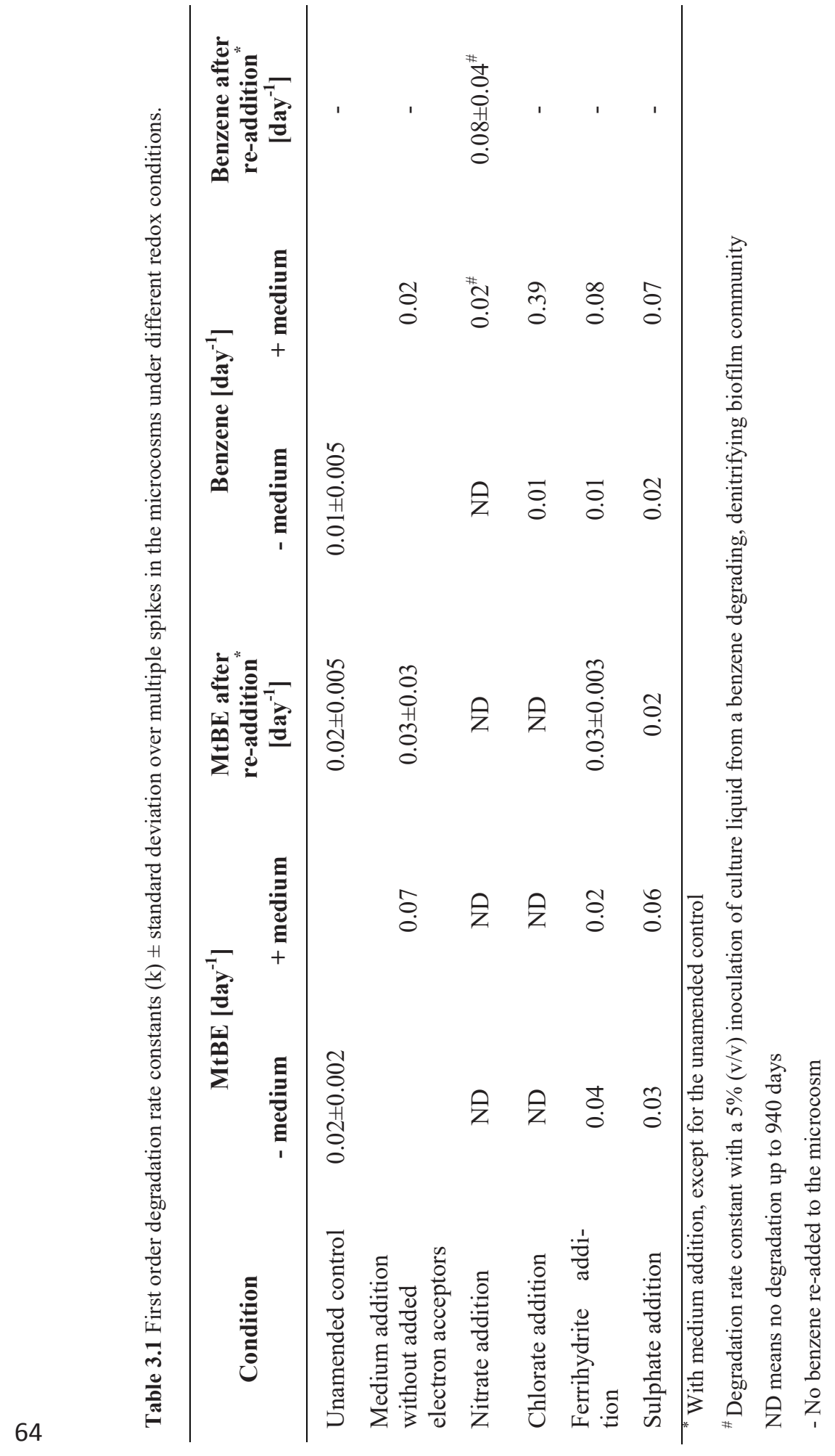


EtBE did not show any degradation after 1140 days in the microcosms under different redox conditions, even after depletion of MtBE and benzene.

MtBE was re-added to the different microcosms after depletion, and an increase in TBA concentration was observed in medium without added electron acceptors, ferrihydritereducing or unamended microcosms corresponding to a percentage of $8 \%, 30 \%$ and $19 \pm 5 \%$, respectively, compared to the concentration of MtBE degraded. The MtBE and TBA concentration profile under natural conditions is shown in Figure 3.1. This TBA increase leveled out to the initial TBA concentration after about 200 days. Complete TBA mineralization has not been proven. After a second MtBE dosage, the TBA concentration increase corresponded to $1 \%, 34 \%$ and $24 \pm 7 \%$ of the MtBE degraded in medium, ferrihydritereducing and unamended microcosms, respectively. The TBA increase was leveled out for this second dosage after 80 days.

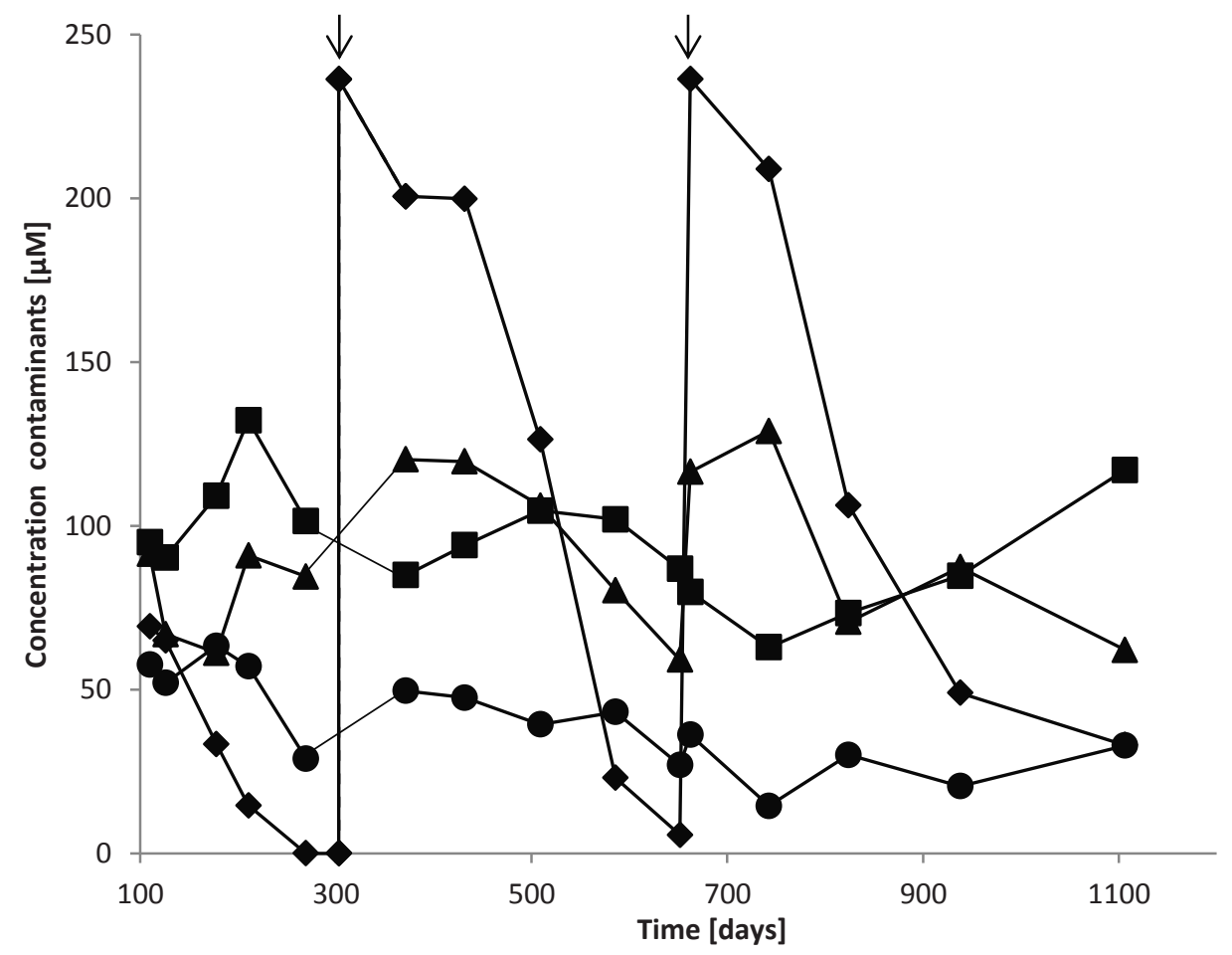

Figure 3.1 Concentration profile under natural conditions of a mixture of MtBE (diamonds), EtBE (squares), TBA (triangles) and benzene (circles). The arrows indicate a re-addition or MtBE. 


\section{Chapter 3.}

Benzene was degraded in unamended controls and with the addition of medium without added electron acceptors, chlorate, ferrihydrite or sulphate, with first order degradation rate constants between 0.01 to 0.02 day $^{-1}$ (Table 3.1). In the presence of medium, the benzene first order degradation rate constants were significantly higher than in the absence of medium under chlorate $\left(0.39\right.$ day $\left.^{-1}\right)$, ferrihydrite $\left(0.08\right.$ day $\left.^{-1}\right)$ or sulphate-reducing conditions $\left(0.07\right.$ day $\left.^{-1}\right)$ (Table 3.1). No degradation with nitrate was observed. The electron balance indicated that more electrons were used to reduce chlorate to chloride than were released by the oxidation of benzene (Table S3.3). Initially no benzene degradation was observed in the nitrate-reducing microcosms. Supply of a 5\% liquid inoculum from a benzene degrading, denitrifying biofilm culture grown on benzene and nitrate with medium resulted in an average degradation rate constant of $0.08 \pm 0.04$ day $^{-1}$ [57]. Over two dosages of $100 \mu \mathrm{M}$ benzene, on average 5.6 $\pm 3.1 \mathrm{~mol}$ nitrate was reduced in parallel with degradation of $1 \mathrm{~mol}$ benzene. No increased methane concentration was measured.

Total bacterial 16S rRNA genes in the microcosms were on average $2.0 \pm 1.3 \times 10^{6}$ gene cop$\mathrm{ies} / \mathrm{ml}$ sample. The $\mathrm{icm} A$ gene was only detected in the microcosms under nitrate-reducing conditions at normalized counts of $6.7 \times 10^{-5}$ and $1.6 \times 10^{-5}$ in the absence or presence of medium, respectively. The $a b c A$ gene was detected in 14 out of 20 microcosms at a normalized count of $5.0 \pm 5.2 \times 10^{-3}$. In the microcosms with addition of medium and the microcosm with groundwater plus nitrate, $a b c A$ genes were below the detection limit $(<1.1$ copies $/ \mu 1$ sample or $<6.0 \times 10^{-4}$ relative to the total bacterial $16 \mathrm{~S}$ rRNA gene copies). The $a b c A$ gene copy number in the microcosm with medium plus nitrate and reactor inoculum was 5.8 times higher than the bacterial 16S rRNA gene number.

\section{Anaerobic TBA and EtBE degradation during growth on MtBE and EtBE analogues and potential metabolites}

Microcosms with vanillate, ferulate, syringate, isopropanol, methanol, or diethyl ether as growth substrate analogues were prepared to determine whether TBA was cometabolically degraded. TBA was depleted with all substrates tested (Table 3.2). 
Table 3.2 EtBE and TBA depletion[\%] after 83 days in microcosms with samples from the unamended control or medium condition, respectively, plus $5 \mathrm{mM}$ analogue substrate. Initially the MtBE, EtBE and TBA concentration were $50 \mu \mathrm{M}$. At 71 days $5 \mathrm{mM}$ vanillate, ferulate or syringate was readded to the specific medium microcosms.

\begin{tabular}{lcc}
\hline $\begin{array}{l}\text { Analogue } \\
\text { substrate }\end{array}$ & $\begin{array}{c}\text { EtBE depletion } \\
\text { unamended control / medium } \\
{[\%]}\end{array}$ & $\begin{array}{c}\text { TBA depletion } \\
\text { unamended control / medium } \\
{[\%]}\end{array}$ \\
\hline Vanillate & $0 / 0$ & $26.3 / 62.5$ \\
Ferulate & $86.5 / 33.9$ & $93.8 / 45.7$ \\
Syringate & $36.4 / 37.6$ & $25.2 / 35.1$ \\
Isopropanol & $15.7 / 0$ & $18.2 / 22.3$ \\
Methanol & $0 / 0$ & $18.7 / 45.8$ \\
Diethyl ether & $55.8 / 44.7$ & $19.6 / 30.6$ \\
\hline
\end{tabular}

After two re-additions of $5 \mathrm{mM}$ syringate, ferulate or vanillate, to microcosms with medium the cumulative amount of TBA depleted increased to $31.6 \mu \mathrm{M}, 33.6 \mu \mathrm{M}$ and $42.9 \mu \mathrm{M}$, respectively in 136 days (Figure 3.2). The amount of vanillate, ferulate or syringate added was respectively 350,447 or 475 mol per mol of TBA depleted. 


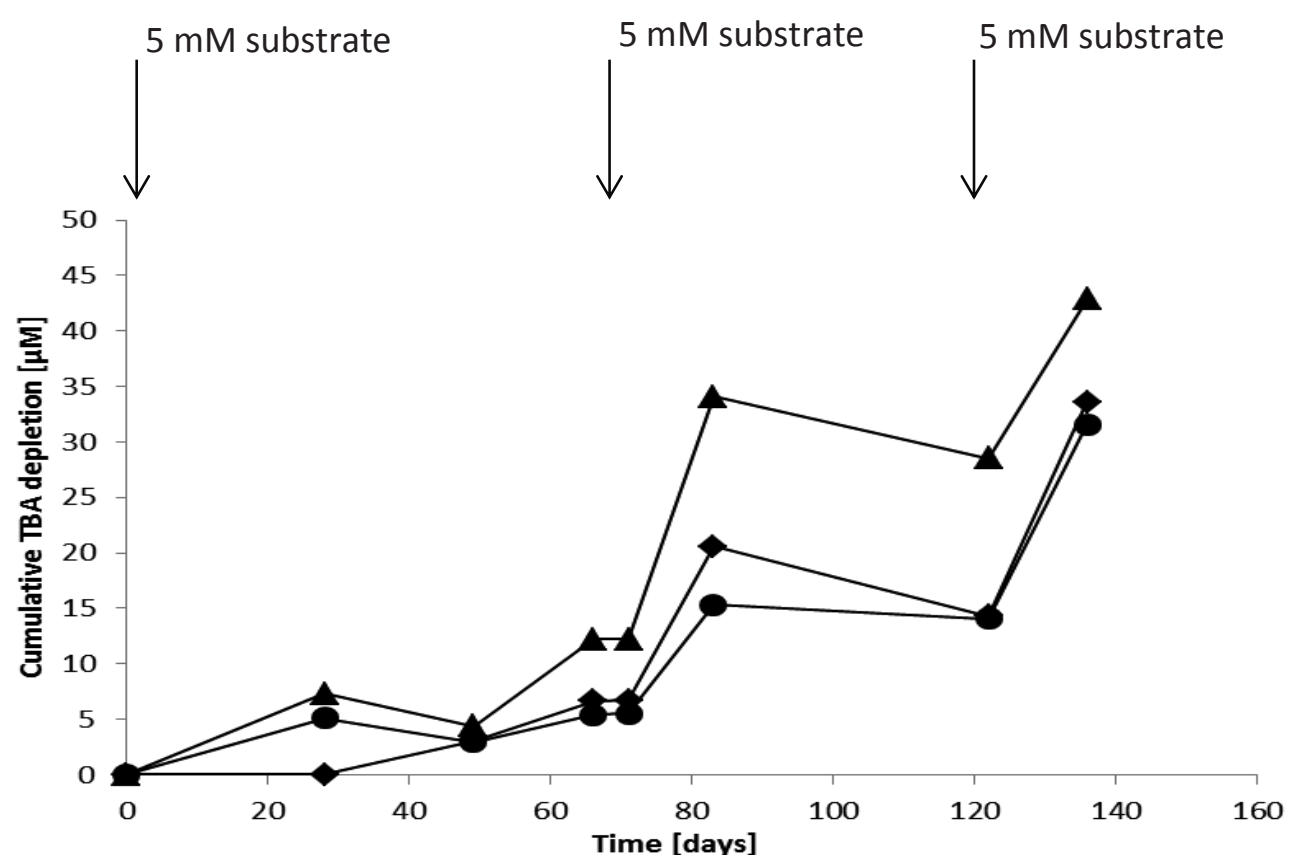

Figure 3.2 The cumulative TBA depletion with vanillate (triangles), ferulate (diamonds) or syringate (circles) in MtBE degrading groundwater microcosms with medium. The arrows indicate a $5 \mathrm{mM}$ dosage of the substrate vanillate, ferulate or syringate, respectively. An initial TBA concentration of $50 \mu \mathrm{M}$ was present in the microcosms.

Also EtBE depletion was observed at significant amounts in microcosms with ferulate (both unamended control and medium), syringate (unamended control and medium), isopropanol (unamended control), or diethyl ether (unamended control and medium), respectively (Table 3.2).

\section{Discussion}

The aims of this study were to: (i) determine the biodegradation potential of the microbial community for MtBE, EtBE, TBA and benzene in a mixture in groundwater from a contaminated location, (ii) determine the effects of electron acceptors, and (iii) determine TBA and EtBE degradation using MtBE and EtBE substrate analogues and potential metabolites. A better understanding of the degradation rates under different redox conditions, and 
cometabolic TBA depletion would enable the design of more effective bioremediation through biostimulation at contaminated sites.

\section{Anaerobic MtBE degradation}

MtBE concentrations decreased strongly in all monitoring wells from 2009 to 2015. The detection of TBA in almost all monitoring wells in 2013 indicates that demethylation is the first step in the MtBE biodegradation in the aquifer [174]. Fractionation of stable carbon isotopes of MtBE and TBA in groundwater in 2009 and 2013 confirmed degradation of MtBE. TBA degradation could not be demonstrated by CSIA.

MtBE was anaerobically degraded in microcosms with groundwater in unamended controls, and after the addition of medium, ferrihydrite or sulphate. This indicates that conditions prevailing at the field site favor MtBE degradation in batch cultures. The MtBE degradation rate constant implemented in the model and representative for the field situation of 0.0007 day $^{-1}$ was 25 times lower compared with the rate observed in the enrichments $\left(0.02 \pm 0.002\right.$ day $\left.^{-1}\right)$. Although data obtained in batch are only indicative for the field situation, these outcomes suggest the potential of biostimulation and/or augmentation of laboratory enriched microbial communities to increase the MtBE degradation rate in the field. The first order MtBE degradation rate constant of $0.02 \pm 0.002$ day $^{-1}$ in the unamended control was similar compared with previous rate constants found of 0.01-0.09 day ${ }^{-1}$ in methanogenic microcosms $[23,159,160]$. The first order MtBE degradation rate constants obtained in this study under sulphate reducing conditions of 0.03 day $^{-1}$ and 0.06 day $^{-1}$ in the absence and presence of medium, respectively, are comparable with a previous microcosm rate constant of $0.031 \pm 0.007 \mathrm{day}^{-1}$ [160]. The TBA concentration did not stochiometrically increase with the amount of MtBE degraded in the microcosms with sulphate. This suggests that MtBE may be completely oxidized to $\mathrm{CO}_{2}$ or other metabolites were formed, which were not detected with the GC-FID. The electrons released from MtBE and benzene degradation were in the range of 60 to $83 \%$ compared with the electrons used to reduce sulphate to sulphide. Somsamak et al. (2006) and Youngster et al. (2008) indicated that in their experiments anaerobic MtBE degradation was not directly coupled to sulfidogenesis [88, 159]. They hypothesize that, MtBE degrading organisms might have produced acetate which was used as a carbon source for sulphate-reducing organisms. Up to our knowledge, this study found the highest first order MtBE degradation rate constant under ferrihydrite reducing conditions of $0.04 \mathrm{day}^{-1}$. Landmeyer and colleagues (1998) reported a first order 


\section{Chapter 3.}

rate constant of $0.0002 \mathrm{day}^{-1}$ under iron-reducing conditions in microcosms [175]. In the ferrihydrite microcosms much more benzene and MtBE was consumed in comparison to the amount of ferrihydrite that was reduced. This indicates that ferrihydrite reduction may not be the predominant electron accepting reaction. The electron imbalance could also be attributed to a lack of complete oxidation of benzene and $\mathrm{MtBE}$ to $\mathrm{CO}_{2}$. The fact that there was no iron(II) production in the sterile controls suggests that abiotic ferrihydrite reduction did not compromise/influence the measurements.

We hypothesized that icmA gene products might also be relevant in anaerobic MtBE degradation since (i) cobalamin-dependent enzymes are important in the metabolism of many anaerobes, and (ii) no oxygenases are needed for the transformation of 2-HIBA to 3hydroxybutyryl-CoA and the further metabolism of this intermediate [176]. In the field, low concentrations of icmA genes were detected in three out of 14 monitoring wells. In microcosms, icmA was only detected under nitrate reducing conditions at low normalized counts of $6.7 \times 10^{-5}$ and $1.6 \times 10^{-5}$. This low icmA concentration suggests that 2-hydroxyisobutyrate is not, or, slowly degraded. It may also be that under anaerobic conditions other isobutyrylCoA mutase enzymes were active, which were not detected by the applied icmA assay [173], or that MtBE and TBA were degraded via alternative metabolic pathways, not involving isobutyryl-CoA mutase.

\section{Anaerobic EtBE degradation}

No significant anaerobic degradation of EtBE was detected in the microcosms under different redox conditions, even after depletion of the (apparently) more easily degradable substrates MtBE and benzene. This result is in line with recent studies indicating the lack of anaerobic EtBE degradation [20, 162]. However, microcosms with ferulate, syringate, isopropanol and diethyl ether, demonstrated EtBE depletion of up to $86.5 \%$ of the initial concentration after 83 days. This suggests that bacteria capable of cometabolic EtBE degradation might be present at this location. We recommend additional research to confirm the possible cometabolic EtBE degradation during growth on methoxylated and ethoxylated substrates.

\section{Anaerobic cometabolic TBA depletion}

Interestingly, TBA concentrations did not stoichiometrically increase in parallel with MtBE depletion in the field, nor in the microcosm experiment. The initial TBA increase after the 
re-addition of MtBE and the subsequent TBA decrease during MtBE degradation to its initial concentration in the microcosms suggests cometabolic TBA degradation. It is feasible that TBA was not depleted further after the primary growth substrate was consumed. This is in line with previous studies observing simultaneous anaerobic MtBE and TBA degradation (Table 3.3). A strong TBA decrease in microcosms with vanillate, ferulate or syringate, indicates cometabolic degradation with MtBE analogue substrates. Possibly TBA degradation was catalysed by enzymes induced for demethoxylation, as was shown previously under aerobic conditions [177]. It is also feasible that TBA degradation is mediated by enzymes responsible for metabolism of tert-alcohol intermediates, which are formed during degradation of methoxylated aromatics. Re-addition of substrate analogues to the medium microcosms doubled the amount of TBA depleted, indicating that these compounds indeed induced cometabolic TBA depletion. Without the addition of these substrates, TBA was not depleted below its initial concentration of $50 \mu \mathrm{M}$, and EtBE was not degraded at all. The amount of vanillate $(350 \mathrm{~mol})$, ferulate $(447 \mathrm{~mol})$ or syringate $(475 \mathrm{~mol})$ was added in excess to degrade $1 \mathrm{~mol}$ of TBA. Therefore, the substrate concentration used in this study is rather high compared with a previously reported MtBE cometabolic degradation study using $1.6 \mathrm{~mol}$ vanillate or $1.7 \mathrm{~mol}$ syringate to degrade $1 \mathrm{~mol}$ of MtBE [88]. Further laboratory experiments will be important to test whether lower substrate concentrations can be used to cometabolically degrade TBA. Addition of the analogue growth substrates to the batches may have enriched microorganisms with enzymes catalysing MtBE, EtBE and TBA transformation. A previous study has demonstrated cometabolic MtBE degradation using syringate, vanillate and guaiacol [88]. It was suggested that acetogenic bacteria capable of degrading methoxylated aromatics may be responsible for this enhanced MtBE degradation [88]. Anaerobic TBA degradation has also been observed in anaerobic microcosms without the addition of MtBE [32]. However, these microcosms contained aquifer sediment heavily contaminated with MtBE, suggesting residual MtBE adsorbed to the sediment might have caused possible cometabolic TBA degradation. 
Chapter 3.

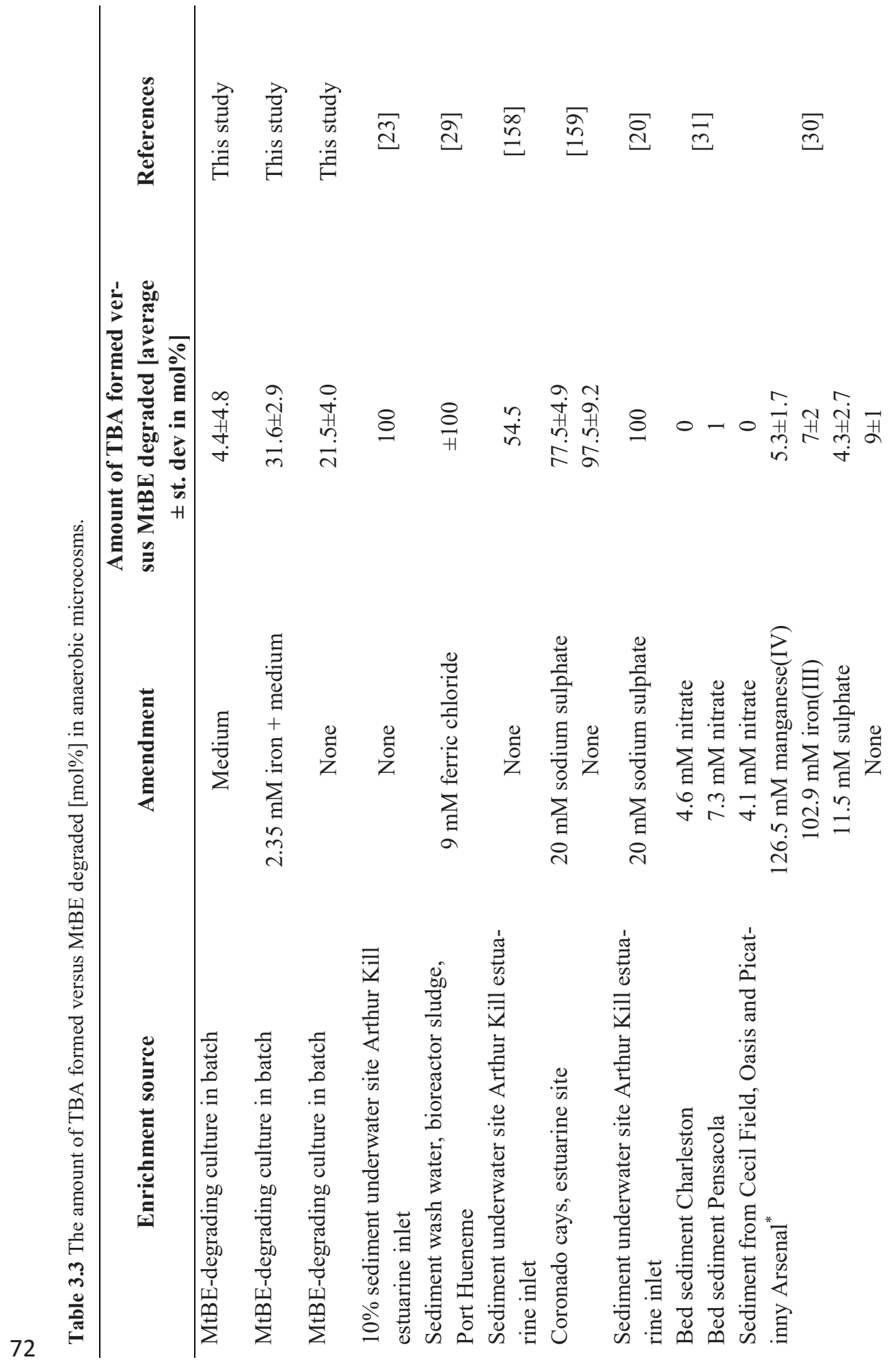


Anaerobic degradation of a mixture of MtBE, EtBE, TBA and benzene

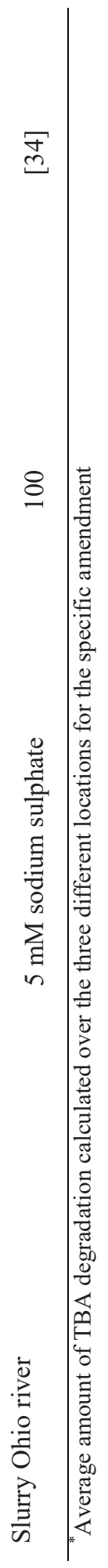




\section{Chapter 3.}

\section{Anaerobic benzene degradation}

The benzene degradation rate constant implemented in the model and representative for the field situation of 0.0007 day $^{-1}$ was 14 to 557 times lower than the first order rate constants measured in microcosms. The benzene first order degradation rate constants in microcosms under chlorate, ferrihydrite and sulphate reducing conditions were higher in the presence of medium. This implies that organisms responsible for benzene degradation were stimulated by nutrients or vitamins supplied with the medium to increase the benzene degradation rate. Initially, benzene was not degraded in microcosms under nitrate reducing conditions. After a $5 \%(\mathrm{v} / \mathrm{v})$ inoculation of culture liquid from a benzene degrading, denitrifying biofilm community a first order benzene degradation rate constant of $0.08 \pm 0.04 \mathrm{day}^{-1}$ was obtained. This rate constant is lower compared with a rate constant of $0.52 \pm 0.03$ day $^{-1}$ found in a previous microcosm study with this biofilm community containing a dense inoculation of biofilm material from the glass walls of the continuous culture [43]. The stoichiometry of anaerobic benzene degradation, $1 \mathrm{~mol}$ benzene consumed per 5.6 $\pm 3.1 \mathrm{~mol}$ nitrate reduced, was similar to that found previously [43]. The incomplete electron recovery of benzene degradation with chlorate or sulphate indicates that electrons released from the relatively high concentration of DOC of $32 \mathrm{mg} / \mathrm{l}$ were used to reduce these acceptors in our microcosms. No increased methane concentration was measured in these microcosms, indicating that methanogenesis was not involved in benzene degradation.

The $a b c A$ gene associated with benzene carboxylation was detected in all monitoring wells and microcosms, albeit in low numbers. The normalized $a b c A$ gene numbers in the microcosms under different redox conditions $\left(5.0 \pm 5.2 \times 10^{-3}\right)$ were higher than the gene numbers measured in the field $\left(1.2 \pm 2.4 \times 10^{-4}\right)$. This suggests that the organisms responsible for benzene degradation through carboxylation were enriched in the microcosms by the addition of nutrients and/or electron acceptors. Groundwater from a leachate-contaminated aquifer polluted with BTEX contained on average $2.4 \times 10^{-4}$ normalized counts of the $b s s A$ gene that codes for benzylsuccinate synthase, an enzyme that is important in toluene degradation [133]. This relative number of $b s s A$ genes is similar to the $a b c A$ gene count in this study. Our results suggest that bioaugmentation and biostimulation can be used to enhance the anaerobic benzene degradation rate at this location. These results are in line with a previous study indicating that benzene is degraded through an initial carboxylation, and the responsible organisms may be stimulated by the addition of nutrients and nitrate [57]. Inter- 
estingly, the microcosm containing nitrate plus medium and an inoculum from a benzenedegrading, denitrifying biofilm culture showed an $a b c A$ gene count of 5.8 times higher than the bacterial $16 \mathrm{~S}$ rRNA gene number. This high $a b c A$ gene count could be due to (i) multiple $a b c A$ gene copies in the DNA of the biofilm culture or (ii) the possibility of the $a b c A$ gene being mobile due to flanking transposon sequences that were observed on the same contig in a metagenome dataset derived from the chemostat biofilm (unpublished data).

\section{Mixture experiment}

Addition of nitrate plus a benzene degrading community and chlorate (with and without medium) stimulated benzene degradation, but hindered MtBE, EtBE and TBA degradation. TBA degradation may be significantly decreased by the presence of benzene in aerobic microcosms $[165,178]$, while other studies indicated no effect of benzene on MtBE and TBA degradation in microcosms [179]. No EtBE degradation was observed in the mixture without substrate analogues. This result is in line with previous studies indicating (i) that under both aerobic and anaerobic conditions, structurally related compounds can have a negative effect on the degradation rates [180] or (ii) that MtBE might be preferentially degraded [181]. Gunasekaran and colleagues reported inhibition of EtBE degradation in the presence of benzene, toluene and xylene [182]. Somsamak and colleagues also reported a lack of EtBE degradation in a mixture of MtBE, EtBE and tert-amyl ether (TAME) [20]. In this study, a stoichiometric increase of TBA with the degradation of MtBE was observed under sulphate-reducing conditions.

Addition of nitrate with a $5 \%(\mathrm{v} / \mathrm{v})$ benzene degrading culture inoculation and chlorate to the microcosms stimulated benzene degradation. For MtBE, EtBE and TBA, electron acceptors such as nitrate appeared to hinder the degradation. Growth substrate analogues stimulated degradation. Cometabolic TBA depletion with MtBE and different methoxylated substrate analogues was indicated in this study. The addition of methoxylated compounds such as syringate, vanillate and ferulate may be a useful method to enhance anaerobic MtBE, EtBE and TBA degradation in situ. Further field studies and dedicated pilot testing could help to elucidate the potential of cometabolic TBA, MtBE and EtBE degradation. The increasing likelihood of encountering mixtures of fossil-based and biobased fuels as cocontaminants in groundwater may require novel or sequential bioremediation approaches. This study describes that biostimulation with substrate analogues, me- 
Chapter 3.

dium and/or nitrate, chlorate, ferrihydrite and sulphate and bioaugmentation approaches can potentially accelerate the degradation of MtBE, EtBE, TBA and benzene. 


\section{Supplementary Figures and Tables}

\section{Carbon Stable Isotope Analysis method 2009}

For stable isotope analysis of MTBE and TBA, extraction from the water phase was carried out using the Stir Bar Sorptive Extraction (SBSE) technique [183]. The stir bar was loaded with compounds, where after the compounds were thermally extracted from the stir bar in a thermal desorption unit $275^{\circ} \mathrm{C}$ (TDU, Gerstel, Germany) and introduced into the GC using a Cold Injection System injector (CIS, Gerstel, Germany). This allows measurement of stable isotope ratios of compounds at low concentration. The gas chromatograph (Agilent 6890 series) was equipped with an AT-1 column $(30 \mathrm{~m} \times 0.32 \mu \mathrm{m} \times 5 \mu \mathrm{m})$. The GC temperature program was held at $50^{\circ} \mathrm{C}$ for $5 \mathrm{~min}$, increased to $120^{\circ} \mathrm{C}$ at a rate of $10^{\circ} \mathrm{C} / \mathrm{min}$, isothermal at $5 \mathrm{~min}$, followed by an increase to $300^{\circ} \mathrm{C}$ at a rate of $35^{\circ} \mathrm{C} / \mathrm{min}$. After combustion to $\mathrm{CO}_{2}$, the stable carbon isotope ratios were determined on a Delta $\mathrm{V}$ isotope ratio mass spectrometer (Thermo Finnigan, Bremen, Germany). 
Chapter 3.

Table S3.1 Overview of the characteristics of the groundwater used to prepare microcosms in 2009 and 2015.

Parameter

Unit

2009

2015

\begin{tabular}{lccc}
\hline Temperature & ${ }^{\circ} \mathrm{C}$ & 12 & 11 \\
$\mathrm{pH}$ & & 7.5 & 6.9 \\
Conductivity & $\mu \mathrm{S} / \mathrm{cm}$ & 2080 & 2040 \\
Oxygen & $\mathrm{mg} / \mathrm{l}$ & 0.3 & 1.1 \\
Dissolved organic carbon & $\mathrm{mg} / \mathrm{m}$ & 17.0 & 32.0 \\
Nitrite & $\mathrm{mg} / \mathrm{mg} / \mathrm{c}$ & $<0.03$ & $<0.01$ \\
Nitrate & $\mathrm{mg} / \mathrm{l}$ & 8.3 & $<0.05$ \\
Sulphate & $\mathrm{mg} / 1$ & $\mathrm{NA}$ & 1.8 \\
Iron (total) & & & 21.0 \\
\hline
\end{tabular}

NA means no iron (total) measured 
Anaerobic degradation of a mixture of MtBE, EtBE, TBA and benzene

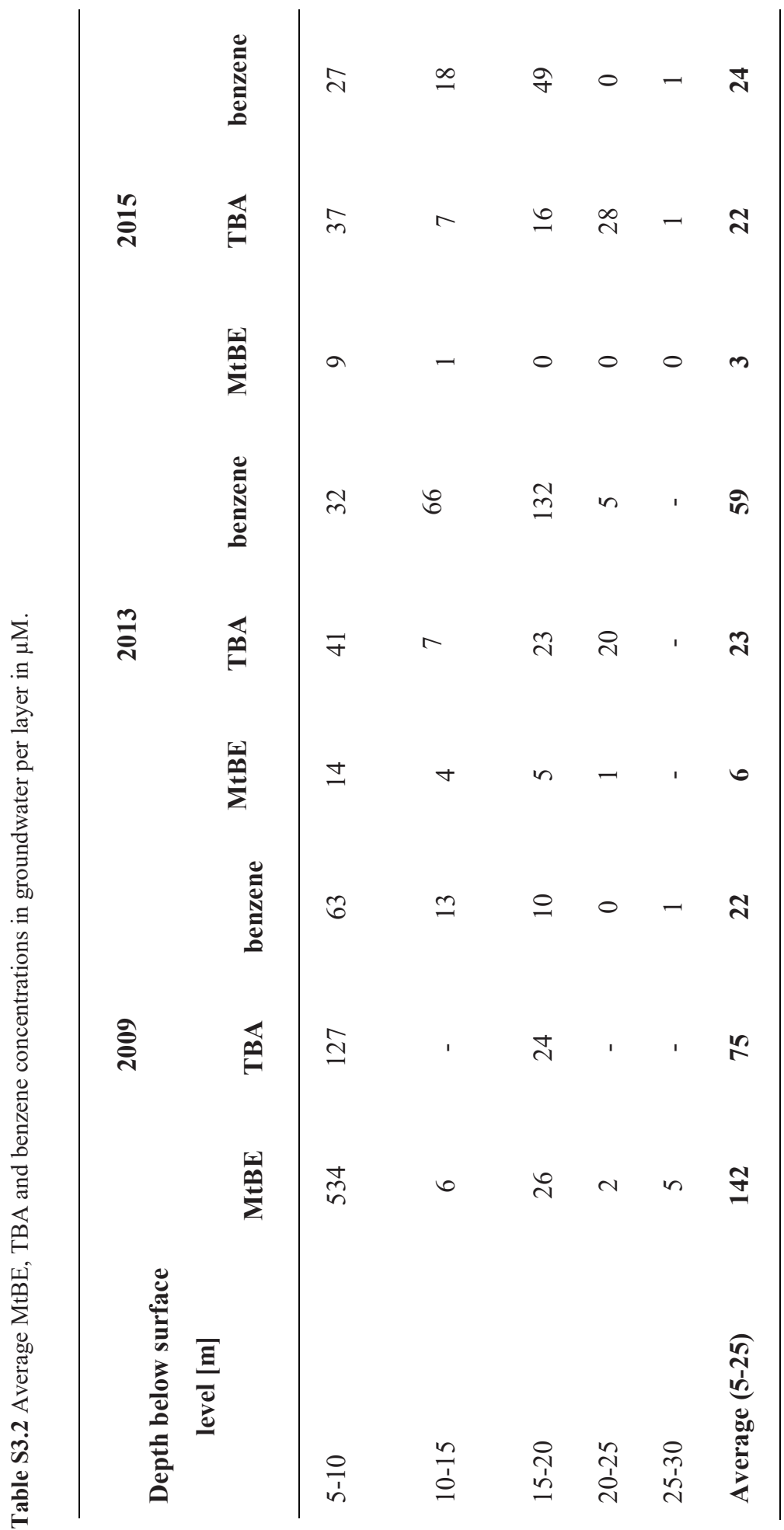


Chapter 3.

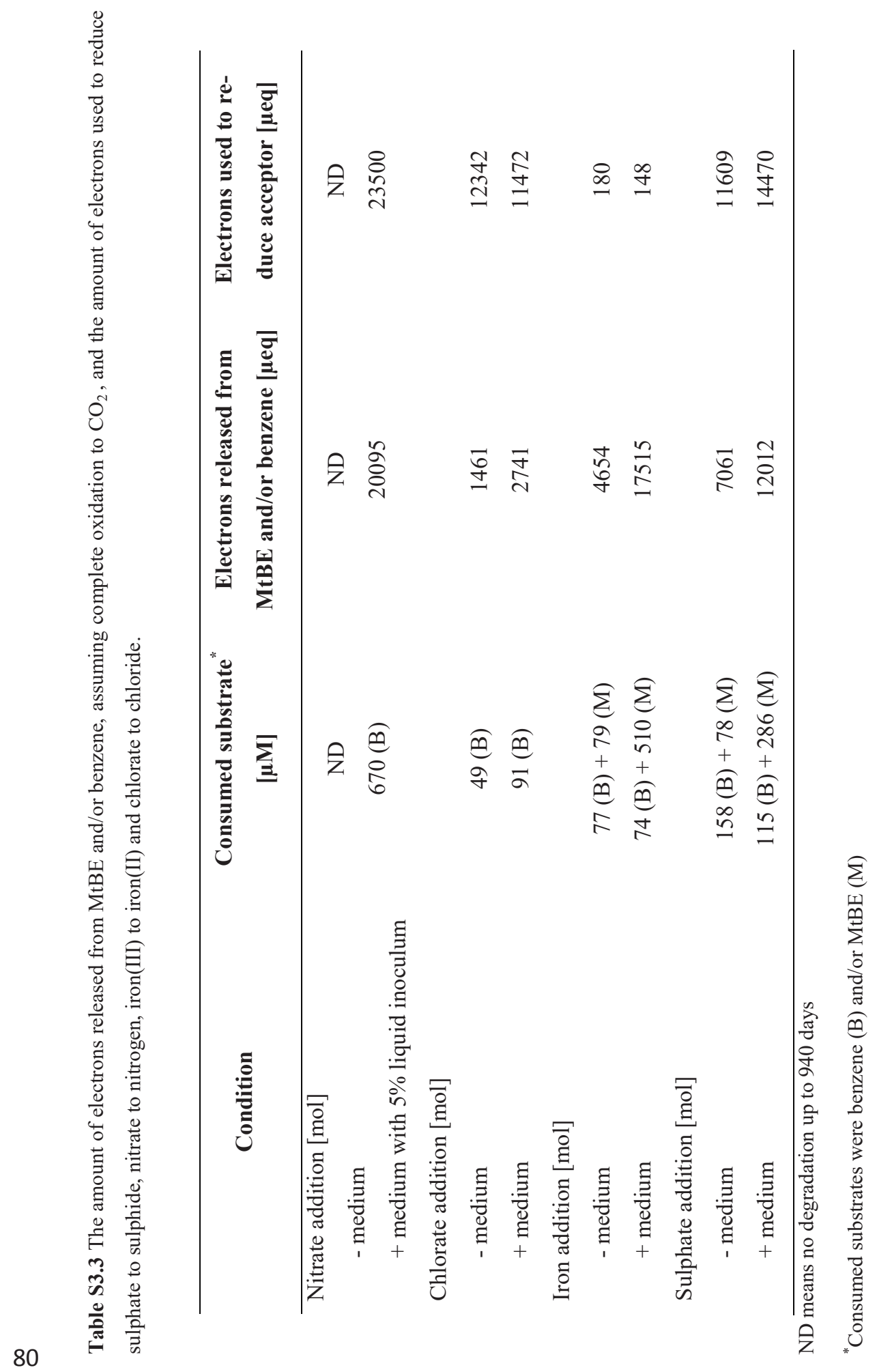


Anaerobic degradation of a mixture of MtBE, EtBE, TBA and benzene
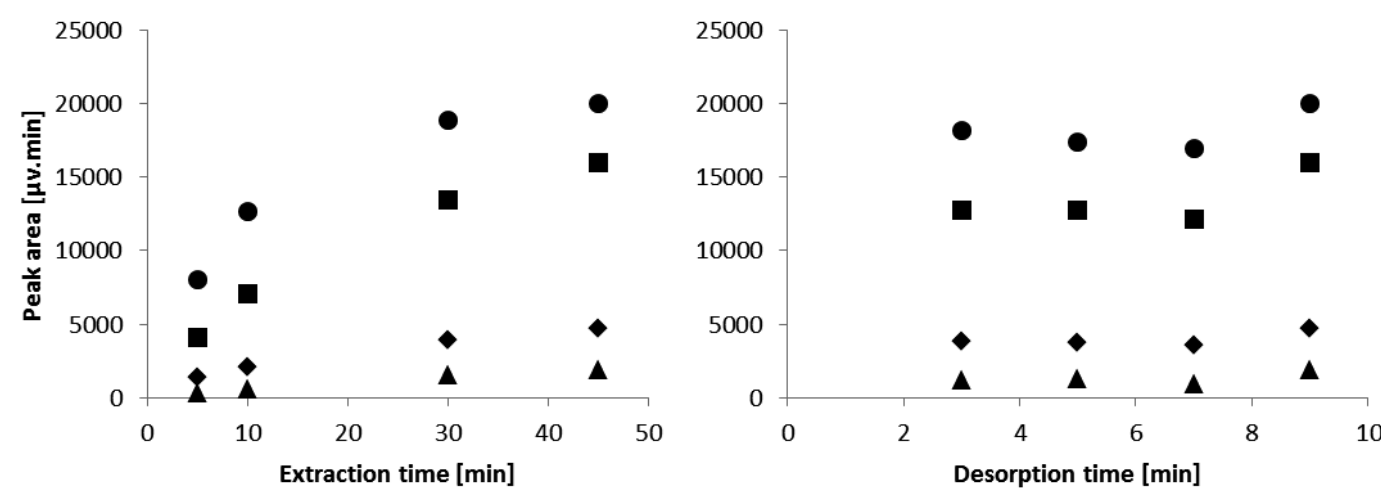

Figure S3.1 Determination of the optimal extraction and desorption time for the GC-FID SPME analysis for MtBE (diamonds), EtBE (squares), TBA (triangles) and benzene (circles). 
Chapter 3. 


\section{Chapter 4. Ethyl tert-butyl ether (EtBE) degradation by an algal-bacterial culture}

Marcelle J. van der Waals, Caroline Plugge, Marion Meima-Franke, Pieter de Waard, Paul

L.E. Bodelier, Hauke Smidt and Jan Gerritse

A version of this chapter has been submitted for publication 


\section{Chapter 4.}

\section{Abstract}

EtBE is a fuel oxygenate that is partially produced from (bio)ethanol and which replaces the fossil-based MtBE. Biodegradation of EtBE can reduce the environmental and human health risks after accidental release. In this study an algal-bacterial culture was used to assess the potential for EtBE degradation. Oxygen produced by the algae Scenedesmus and Chlorella was used by bacteria to degrade EtBE using a P450 monooxygenase and isobutyryl-CoA mutase. The metabolites formed during the micro-oxic EtBE degradation were tert-butyl alcohol (TBA), ethanol and $\mathrm{CO}_{2}$ as determined using ${ }^{13} \mathrm{C}$ nuclear magnetic resonance spectroscopy (NMR) and gas chromatography. Stable isotope probing (SIP) with ${ }^{13} \mathrm{C}_{6}$ labelled EtBE in a fed-batch vessel showed no significant difference in community profiles of the ${ }^{13} \mathrm{C}$ and ${ }^{12} \mathrm{C}$ enriched DNA fractions, with representatives of the families Halomonadaceae, Shewanellaceae, Rhodocyclaceae, Oxalobacteraceae, Comamonadaceae, Sphingomonadaceae, Hyphomicrobiaceae, Candidatus Moranbacteria, Omnitrophica, Anaerolineaceae, Nocardiaceae, and Blastocatellaceae being predominant. This is the first study describing micro-oxic degradation of EtBE by an algal-bacterial culture. This algalbacterial culture has advantages compared with conventional aerobic treatments, including a lower risk of EtBE evaporation and no need for external oxygen supply. This study provides novel leads towards future possibilities to implement algal-bacterial consortia in fieldscale groundwater or wastewater treatment.

\section{$\underline{\text { Introduction }}$}

Fuel oxygenates such as methyl tert-butyl ether (MtBE) and ethyl tert-butyl ether (EtBE), were incorporated into gasoline in the 1970s because of their high octane number and their effect on the reduction of greenhouse gas emissions [184]. In Europe, MtBE is being replaced by EtBE produced from the renewable resource (bio)ethanol and isobutylene to comply with the European goal of $10 \%$ biobased fuel addition in 2020. EtBE is increasingly used to replace MtBE with a total EtBE consumption of 3 million metric tons in Europe and Japan in 2011 [12].

Fuel oxygenates enter the groundwater through spillages during production, distribution and storage. Due to the high EtBE water solubility of $12 \mathrm{~g} / 1$, the poor biodegradation and low odour and taste thresholds in water of 13 and $47 \mu \mathrm{g} / \mathrm{l}$, respectively, EtBE imposes a risk in groundwater $[12,185]$. Despite the increasing EtBE consumption, little is known regard- 
ing its environmental fate. The actual level of EtBE groundwater contamination is not known. A Dutch study monitoring 37 groundwater collection areas, showed two areas with EtBE being detected at 0.1 and $1 \mu \mathrm{g} / \mathrm{l}$, respectively [13]. Partly because of the results of this study, the Dutch Ministry of Housing, Spatial Planning and the Environment set an EtBE remediation value of $1 \mu \mathrm{g} / 1$ within drinking water collection areas and $15 \mu \mathrm{g} / \mathrm{l}$ outside drinking water collection areas [186]. Also in the US several EtBE groundwater contaminations were detected, because of usage in the past [187]. EtBE concentrations between 0.35 $7500 \mu \mathrm{g} / 1$ were measured in groundwater beneath underground leaking storage tanks.

There is little information on the biological transformation and removal of EtBE in contaminated soils, aquifers and wastewater. In general, EtBE is recalcitrant due to its tertiary carbon atom and ether bond [22]. A few bacterial strains have been isolated that are able to aerobically degrade EtBE $[12,188,189]$. Aerobic EtBE degradation is initiated by cytochrome P450 monooxygenase encoded by the ethRABCD gene cluster, found in Grampositive EtBE-utilizing strains of the genera Rhodococcus, Gordonia and Mycobacterium [190-193]. Oxygen, however, is often rapidly depleted in contaminated groundwater and wastewater by aerobic (micro)organisms using easily degradable substrates as electron donor [18]. Therefore, anaerobic EtBE degradation is highly important. A previous study reported lack of anaerobic EtBE degradation [20]. However, Yeh and Novak [21] found anaerobic EtBE degradation in soils under denitrifying and methanogenic conditions. Compound-specific stable isotope analysis (CSIA) revealed insignificant carbon isotope fractionation, but low hydrogen isotope fractionation of up to $+14 \%$ along an anoxic EtBE plume suggesting anaerobic degradation of EtBE [22].

Algae and bacteria have coexisted since the early stages of evolution. It has been shown that algae and bacteria synergistically affect each other's physiology and metabolism. Algal-bacterial interactions include mutualism, commensalism but also parasitism [194]. A previous study indicated the presence of viable algae down to $225 \mathrm{~m}$ in the subsurface indicating that algae may be able to survive or thrive in anoxic groundwater [195]. It was found that a symbiotic algal-bacterial system could improve the bacterial MtBE degradation due to higher dissolved oxygen concentrations generated by photosynthesis [196]. Algalbacterial interactions in wastewater treatment plants have been shown to be effective for treating contaminants such as acetonitrile, thiocyanate and atrazine [197-199]. 


\section{Chapter 4.}

The aims of the present study were to determine the capacity, pathway and phylogenetic composition of the microbial community involved in EtBE degradation in anoxic groundwater from a contaminated location. The EtBE degradation pathway was determined by ${ }^{13} \mathrm{C}$ nuclear magnetic resonance (NMR) spectroscopy. The phylogenetic composition was assessed using generic high-throughput analysis of $16 \mathrm{~S}$ ribosomal RNA (rRNA) genes through next generation technology sequencing (i.e. Illumina MiSeq) of PCR-barcoded amplicons. Sequencing was done of amplicons obtained from ${ }^{12} \mathrm{C}$ and ${ }^{13} \mathrm{C}$ enriched DNA fractions after stable isotope probing (SIP) using a ${ }^{13} \mathrm{C}_{6}$ labelled EtBE degrading microbial community in a fed-batch reactor in order to identify microbial taxa involved in key catabolic reactions.

\section{Materials and Methods}

Anoxic EtBE contaminated groundwater from a gasoline fuelling station site in Amsterdam, the Netherlands, was used to inoculate a 3 litre fed-batch reactor (Applikon, Delft, the Netherlands) and microcosms. Groundwater from this site, with a level of $2.25 \mathrm{~m}$ below ground surface, had an electrical conductivity of $1.2 \mu \mathrm{S}$ and $\mathrm{pH}$ of 7.2 with a nitrate and sulphate concentration of $25 \mathrm{mg} / 1$ and $38 \mathrm{mg} / 1$, respectively. At the impacted area, groundwater was pumped from 2.4-3.4 m below ground surface using new polyethylene tubing, a new marprene pumping tube and 20 L flasks (Nalgene, New York, USA), to prepare microcosms and a fed-batch reactor. In this groundwater mineral oil $(<50 \mu \mathrm{g} / \mathrm{l})$, xylene $(0.2 \mu \mathrm{g} / \mathrm{l})$, $\operatorname{MtBE}(2.6 \mu \mathrm{g} / \mathrm{l})$ and $\operatorname{EtBE}(250 \mu \mathrm{g} / \mathrm{l})$ were present.

\section{EtBE degradation under different redox conditions}

Microcosms were prepared as previously described under a $80 / 20 \% \mathrm{~N}_{2} / \mathrm{CO}_{2}(\mathrm{v} / \mathrm{v})$ flow in $250 \mathrm{ml}$ serum bottles (Glasgerätebau Ochs GmbH, Bovenden, Germany) and crimp-sealed with viton rubber stoppers (Rubber BV, Hilversum, the Netherlands) and aluminium crimp caps (Grace) [200]. The microcosms contained $90 \mathrm{ml}$ of groundwater amended with $50 \mu \mathrm{M}$ EtBE, because it had been removed during flushing. One series of microcosms each with a different electron acceptor was amended with $10 \mathrm{ml}$ medium [57]. No medium was added to a second series of microcosms with the addition of only electron acceptors. Abiotic control microcosms were autoclaved and contained $100 \mathrm{mg} / 1 \mathrm{HgCl}_{2}$ and $2 \mathrm{mg} / \mathrm{l} \mathrm{NaN}$. Unamended control microcosms, not receiving any medium and/or electron acceptors, were used as representatives for the natural condition. The microcosms were incubated upside 
down and continuously shaken at $100 \mathrm{rpm}$ (Certomat, B|Braun, Melsungen, Germany) in the dark at $20^{\circ} \mathrm{C}$.

\section{Fed-batch reactor}

Two litre of groundwater was added to the reactor vessel. EtBE degradation was activated using 1.5 litre of this groundwater and 0.5 litre of defined medium as described previously, but without nitrate [57]. Subsequently $50 \mu \mathrm{M}$ EtBE was added to the system from a $20 \mathrm{mM}$ autoclaved, anoxic stock solution. Each time EtBE was depleted, $50 \mu \mathrm{M}$ EtBE was replenished from the stock solution. The $\mathrm{pH}$ of the liquid was 6.8 and the temperature in the reactor vessel was maintained at $25{ }^{\circ} \mathrm{C}$. The liquid phase was stirred at $200 \mathrm{rpm}$ and the headspace was maintained at 0.02-0.1 bar $\mathrm{N}_{2} / \mathrm{CO}_{2}(80: 20 \mathrm{v} / \mathrm{v})$ gas, which had been passed over a $460 \mathrm{ml}$ glass column filled with copper flakes $\left(250{ }^{\circ} \mathrm{C}\right)$ and bubbled through a bottle with titanium citrate solution [201] to remove traces of oxygen. The redox potential and oxygen concentration were measured using electrodes submerged in the liquid of the fed-batch reactor (AppliSens, Applikon).

To confirm that the reactor vessel was anoxic and no trace concentrations of oxygen could enter due to for example oxygen diffusion through connection points and tubing, three experiments were performed: (i) adding 5\% (v/v) of hydrogen to the headspace of the vessel, (ii) placing the fed-batch reactor in a gas bag (Glas-Col, IN, USA) with a continuous $\mathrm{N}_{2} / \mathrm{CO}_{2}$ flow and closed with tie wraps and (iii) submerging the fed-batch reactor in a dark bucket filled with tap water.

Two experiments were done to determine the algal-bacterial interaction. First, liquid from the reactor vessel $(10 \mathrm{ml})$ was transferred to a $20 \mathrm{ml}$ headspace vial (Grace, Maryland, USA). After the degradation of $75 \mu \mathrm{M}$ of EtBE, $100 \mu \mathrm{g} / \mathrm{ml}$ cycloheximide was added to inhibit protein synthesis in eukaryotic cells. After 52 days, $2 \mathrm{ml}$ of this liquid was transferred to a $10 \mathrm{ml}$ headspace vial (Grace) and amended with either $0.5 \mathrm{ml} 1 \mathrm{x}$ phosphate buffered saline solution (PBS) or 1x PBS with $5 \mathrm{mM}$ metyrapone (Sigma-Aldrich, Missouri, USA) to inhibit cytochrome P450 monooxygenase. Secondly, liquid from the reactor vessel $(100 \mathrm{ml})$ was incubated in an inverted microcosm under anoxic conditions in the dark at $20^{\circ} \mathrm{C}$, to determine anaerobic EtBE degradation. 


\section{Chapter 4.}

\section{Analyses}

EtBE in headspace samples was measured with a Varian 3800 gas chromatograph (GC) equipped with a flame ionization detector (FID) as previously described [57]. Briefly, the FID detector was set at $300{ }^{\circ} \mathrm{C}$ and the sample was injected at $200{ }^{\circ} \mathrm{C}$ with a split ratio of 5 . The oven temperature program was $3 \mathrm{~min}$ at $50{ }^{\circ} \mathrm{C}$, followed by an increase of $10{ }^{\circ} \mathrm{C} / \mathrm{min}$ to $250{ }^{\circ} \mathrm{C}$ for $2 \mathrm{~min}$. Fed-batch reactor headspace samples of $0.5 \mathrm{ml}$ were taken with a $1-\mathrm{ml}$ Pressure-Lock gas syringe (Alltech, Breda, the Netherlands) and directly injected on the GC-FID. Six standards from 10 to $200 \mu \mathrm{M}$ EtBE in serum bottles with the same headspace/water ratio $(\mathrm{v} / \mathrm{v})$ as in the corresponding fed-batch reactor or microcosms were crimp sealed with viton stoppers and aluminium caps and used for calibration. The coefficient of variation of this GC method was $6.9 \%$ and the EtBE detection limit was $0.1 \mu \mathrm{M}$. In $0.3 \mathrm{ml}$ liquid samples, tert-butyl alcohol (TBA), EtBE and ethanol were measured as previously described using solid phase micro-extraction (SPME) on a GC-FID [200]. Potential metabolites detected with this method include: acetone, isopropanol and N-propanol. Anion analysis was done by liquid chromatography as described previously [200].

\section{Nuclear magnetic resonance spectroscopy (NMR)}

${ }^{13} \mathrm{C}_{6}$ labelled EtBE was purchased from Sigma-Aldrich. The ${ }^{13} \mathrm{C}_{6}$ labelled EtBE contained a ${ }^{13} \mathrm{C}$ labelled TBA impurity of about $50 \%$ before usage. A spike of $50 \mu \mathrm{M}$ EtBE was added to the fed batch reactor vessel from a $22 \mathrm{mM}$ anoxic stock solution. Each time ${ }^{13} \mathrm{C}_{6}$ labelled EtBE was depleted, $50 \mu \mathrm{M}$ was replenished from a $22 \mathrm{mM}$ anoxic stock solution with a total of $150 \mu \mathrm{M}{ }^{13} \mathrm{C}_{6}$ EtBE. Samples of $2 \mathrm{ml}$ were taken at different time points and centrifuged at $10,000 \times \mathrm{g}$ as described by Bui and co-authors [202]. Briefly, $\mathrm{D}_{2} \mathrm{O}(50 \mu \mathrm{l} ; 99.9$ atom $\%$, Sigma-Aldrich) was added to the supernatants $(0.5 \mathrm{ml})$ of the centrifuged samples and subsequently transferred to NMR tubes (Campro Scientific, Berlin, Germany). ${ }^{13} \mathrm{C}$ NMR spectra were recorded at a probe temperature of $300 \mathrm{~K}$ on a Bruker Advance-600 spectrometer equipped with a cryoprobe (Bruker, MA, USA). Chemical shifts were expressed in ppm. The products were identified based on chemical shifts compared with the Scifinder database. One-dimensional ${ }^{13} \mathrm{C}$ spectra were acquired into a time domain of a total of 66000 scans resulting in a measuring time of $18 \mathrm{~h}$, an acquisition time of $0.36 \mathrm{~s}$ and a sweep width of $300 \mathrm{ppm}$. 


\section{DNA extraction, gradient fractionation and molecular analyses}

Biomass was concentrated from $10 \mathrm{ml}$ microcosm or fed-batch samples by vacuum filtration on $0.2 \mu \mathrm{m}$ mixed cellulose ester pore-size filters (Merck Millipore). DNA extraction was done as described previously [200]. Briefly, filters were crushed with a sterilized wooden tooth pick and total DNA was extracted using the MoBio Powerlyzer DNA isolation kit (MoBio, CA, USA).

${ }^{13} \mathrm{C}$-enriched DNA was separated from non-labelled DNA by isopycnic density gradient centrifugation [203]. The gradient buffer was composed of $\mathrm{CsCl}$ (Sigma-Aldrich) with an average density of $1.83 \mathrm{~g} / \mathrm{ml}$ dissolved in a gradient buffer $(0.1 \mathrm{M}$ Tris- $\mathrm{HCl} \mathrm{pH} 8$, $0.1 \mathrm{M} \mathrm{KCl}, 1 \mathrm{mM}$ EDTA) [204]. $500 \mathrm{ng}$ of DNA was used per sample, and centrifugation was carried out in $1.8 \mathrm{ml}$ polyallomer ultracrimp tubes (ThermoScientific, MA, USA) in a micro ultracentrifuge with vertical rotor (Sorvall discovery m120 SE, S120-VT). Centrifugations were done at $60.000 \mathrm{RPM}$ for $72 \mathrm{~h}$ at $20^{\circ} \mathrm{C}$ with minimum acceleration and minimum deceleration. Per sample around 18 fractions of $100 \mu 1$ were collected using RNAse free water with coomassie brilliant blue (Sigma Aldrich). The density of each fraction was determined using an AR200 digital refractometer (Reichert, NY, USA). Nucleic acids were precipitated using $20 \mu \mathrm{g}$ glycogen and 20\% PEG with $1.6 \mathrm{M} \mathrm{NaCl}$ for subsequent quantitative and qualitative community analyses. The fractionated DNA was combined into samples called 'heavy' and 'light', with densities around $1.74 \mathrm{~g} / \mathrm{ml}$ and $1.73 \mathrm{~g} / \mathrm{ml}$, respectively, calculated based on a calibration curve of a $\mathrm{CsCl}$ gradient mixture in water (Figure S4.1). The pooled DNA contained between 1 to 3 fractions, depending on the sample quality.

Total bacterial 16S rRNA genes and icmA genes coding for isobutyryl-CoA mutase were quantified as described previously [57, 200]. Total archaeal 16S rRNA genes were quantified as described by van der Zaan and co-workers using primer pair Arch0025F (5'-CTGGTTGATCCTGCCAG-3') [205] and Arch364R $\left(5^{\prime}-\right.$ TCGCGCCTGCTGCGCCCCGT-3') [206]. A quantitative real-time PCR assay to detect the $e t h B$ gene coding for the cytochrome P450 monooxygenase subunit was performed as described elsewhere [193].

For this study a quantitative real-time PCR assay was designed to detect the $m d p A$ gene coding for MtBE monooxygenase that has been shown to be a key enzyme involved in aerobic MtBE degradation in Methylibium petroleiphilum PM1 using Primer3 software [134, 135, 207]. The designed primer pair F543 (5'-TACTCCTGAGGACTCCAGTT-3') 


\section{Chapter 4.}

and R732 (5'-GTAGAAGACCAGCGAGATCA-3') with TaqMan probe 5'ATTTATTCGACCCTGGCGCC-3' (P621) was evaluated in silico using the publicly accessible NCBI BLAST search tool [136]. The $m d p A$ gene assay was performed in $25 \mu 1$ volumes as described elsewhere [57]. The PCR conditions were the same as described for the icmA gene [200].

\section{S rRNA gene amplicon sequencing and data analysis}

Illumina MiSeq sequencing of amplicons derived from the extracted DNA was used for bacterial community analysis. Briefly, barcoded amplicons covering the V1-V2 region of 16S rRNA genes were generated using a two-step PCR method as described earlier [57, 125]. Pooled amplicons were sequenced by GATC Biotech AG (Constance, Germany) on an Illumina MiSeq platform. 16S rRNA amplicon sequence analysis was done using NGTax [127]. Briefly, paired-end libraries were filtered to retain read pairs with perfectly matching barcodes. 16S rRNA gene sequences were clustered into operational taxonomic units (OTUs) at $>98.5 \%$ sequence similarity. The phylogenetic diversity (PD whole tree) was calculated using Quantitative Insights Into Microbial Ecology (QIIME) [129]. Two different Mock communities used in this analysis had Pearson correlations at genus level of 0.55 with respect to the microbial community theoretically present in the Mock community, which are values routinely observed in other runs using the same set-up. Nucleotide sequence data reported are available at the European Nucleotide Archive (ENA) under accession number PRJEB25329.

\section{Algae growth experiment}

To determine the presence of algae, the fed-batch reactor samples were exposed to daylight (diurnal cycles of eight hours daylight and 16 hours without light) or artificial light (4125 lux continuous light, Philips 15 watt). The microcosms were incubated in front of a window with average daylight of 12 hours. Algal growth tests were done by inoculation of $0.1 \% \mathrm{v} / \mathrm{v}$ fed-batch reactor samples in BG-11 broth (Sigma-Aldrich) and exposed to daylight (average daylight of 12 hours). A $0.1 \% \mathrm{v} / \mathrm{v}$ re-inoculation in BG-11 broth of this algal enrichment exposed to daylight was made anoxic by flushing with $80 / 20 \% \mathrm{~N}_{2} / \mathrm{CO}_{2}(\mathrm{v} / \mathrm{v})$ gas and placed in the dark for determination of ethanol production. 


\section{First order degradation rate constant calculation}

First order degradation rate constants (k) were calculated according to Equation 4.1 [111].

$\mathrm{k}=\left(\frac{\log \left(\mathrm{x}_{1}\right)-\log \left(\mathrm{x}_{2}\right)}{\mathrm{t}_{2}-\mathrm{t}_{1}}\right)$

where $t_{2}-t_{1}$ is the time interval in days. $x_{1}$ is the EtBE concentration at time $t_{1}$ and $x_{2}$ is the EtBE concentration at time $t_{2}$.

\section{$\underline{\text { Results }}$}

\section{EtBE degradation in microcosms under different redox conditions}

Neither growth nor EtBE loss was observed in an autoclaved microcosm with $100 \mathrm{mg} / \mathrm{l}$ $\mathrm{HgCl}_{2}$ and $2 \mathrm{mg} / 1 \mathrm{NaN}_{3}$ in dark or light conditions. EtBE contaminated groundwater from a gasoline fuelling station site (unamended control) and the microcosms under nitrate-, chlorate-, sulphate- and ferrihydrite-reducing conditions did not show EtBE degradation in the dark at $20^{\circ} \mathrm{C}$ after 870 days (degradation less than the coefficient of variation). Under aerobic conditions, EtBE was degraded at a rate of 33.6 $\pm 2.4 \mu \mathrm{M} /$ day. In an unamended microcosm, algal growth was observed after eight weeks when exposed to a day-night light cycle (Figure S4.2). Morphology suggested that the algae belonged to the genera Scenedesmus and Chlorella. The degradation rate of EtBE was $0.8 \pm 0.2 \mu \mathrm{M}$ /day in this algal enrichment (Table 4.1). Oxygen in the headspace increased up to 50\% (v/v) (Figure 4.1). After 54 days the microcosm was placed in the dark after which the oxygen level in the microcosm decreased and EtBE was continuously degraded at a rate of $0.8 \pm 0.2 \mu \mathrm{M} /$ day. In this microcosm, TBA concentrations increased stepwise up to $80 \mu \mathrm{M}$ when three doses of each 50 $\mu \mathrm{M}$ EtBE were added to the microcosm. The ethanol concentration increased up to $35 \mu \mathrm{M}$ in 85 days, after which it decreased. The ethB gene, coding for cytochrome P450 monooxygenase, was not detected in the unamended aerobic control microcosm degrading EtBE. 
Chapter 4.

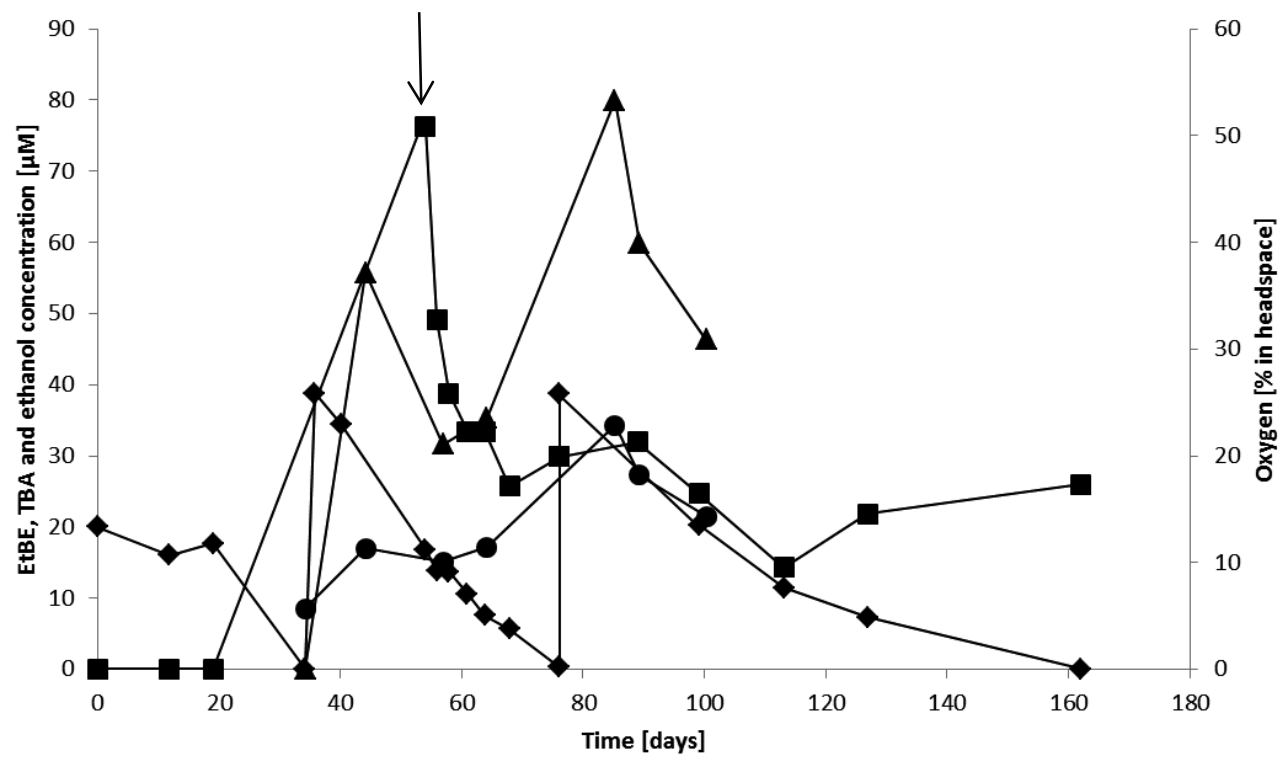

Figure 4.1 Concentrations of EtBE (diamonds), TBA (triangles), ethanol (circles) and oxygen (squares) in the microcosm without the addition of electron acceptor or medium. At time 0 the microcosm was exposed to an ambient day-night light cycle of 8/16 hours. After 54 days the microcosm was placed back in the dark at $20^{\circ} \mathrm{C}$ (indicated by the arrow) 
EtBE degradation by an algal-bacterial culture

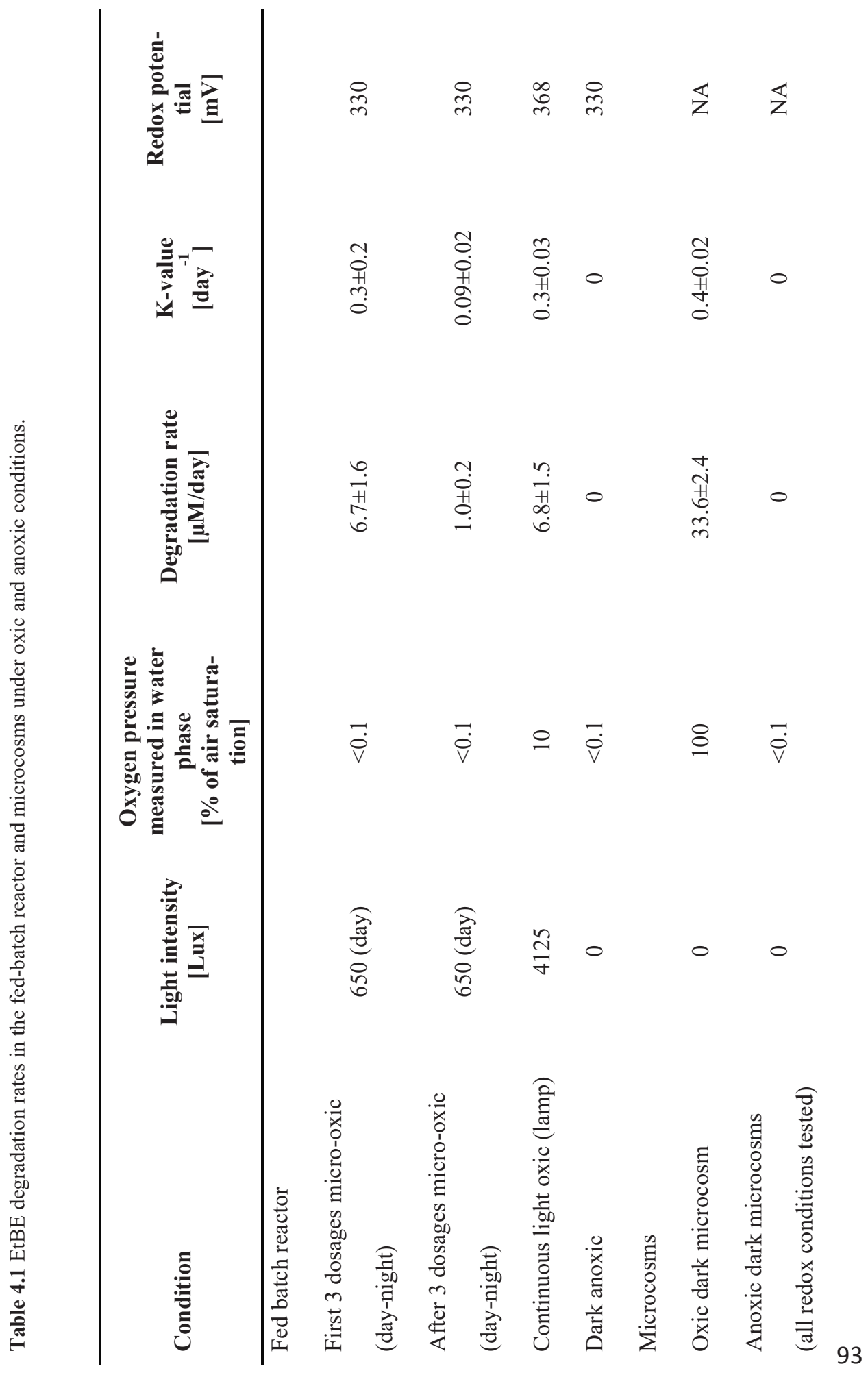


Chapter 4.

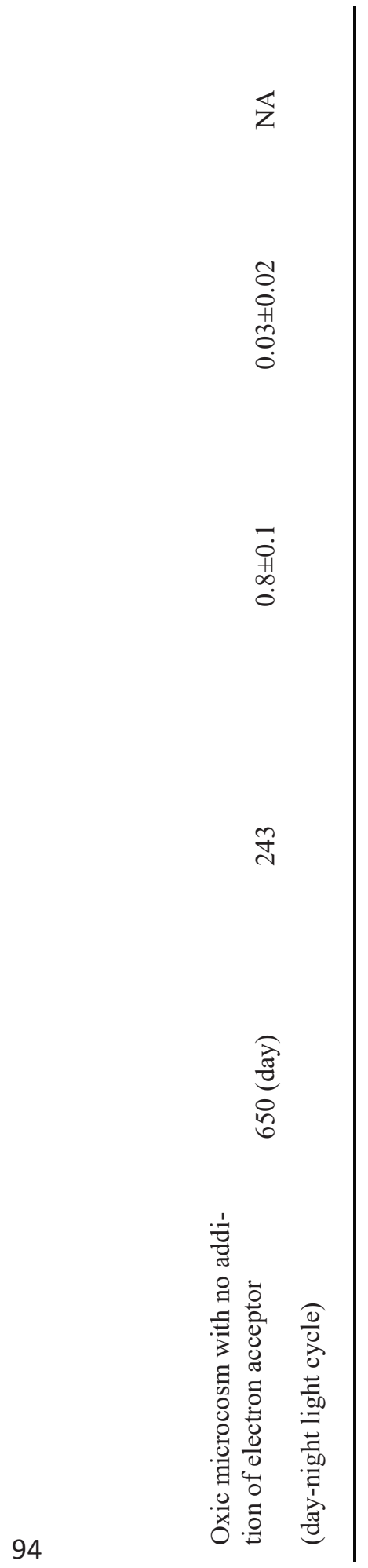


EtBE degradation by an algal-bacterial culture

\section{EtBE degradation in a fed-batch reactor}

\section{EtBE degradation rate}

No EtBE loss was observed in the fed-batch reactor filled with groundwater from the contaminated location when exposed to ambient day/night cycles. Therefore, 0.5 litre of defined medium was added to 1.5 litre of groundwater after 135 days. The liquid in the fedbatch reactor had a redox potential between +330 and $+368 \mathrm{mV}$. The oxygen concentration measured in the liquid was $<0.1 \%$. The microbial community degraded multiple doses of $50 \mu \mathrm{M}$ EtBE with a cumulative total of $1250 \mu \mathrm{M}$ in a day/night cycle. The first-order degradation rate for the first three $50 \mu \mathrm{M}$ EtBE dosages was $6.7 \pm 1.6 \mu \mathrm{M}_{\text {.day }}{ }^{-1}\left(0.3 \pm 0.2\right.$ day $\left.^{-1}\right)$ corresponding to a half-life of approximately 2.3 days (Table 4.1). After three EtBE readditions, the degradation rate decreased to $1.0 \pm 0.2 \mu \mathrm{M} . d a y^{-1}\left(0.09 \pm 0.02\right.$ day $\left.^{-1}\right)$. After exposure of the culture vessel to continuous light (4125 lux), the degradation rate increased to $6.8 \pm 1.5 \mu \mathrm{M}_{\text {.day }}{ }^{-1}\left(0.3 \pm 0.03\right.$ day $\left.^{-1}\right)$. Under these conditions, the oxygen concentration in the liquid phase increased from $<0.1 \%$ to $10 \%$.

At 400 days after the start-up of the fed batch culture and a cumulative dosing of $500 \mu \mathrm{M}$ EtBE, 5\% hydrogen was added to the headspace of the vessel to ensure strictly anoxic conditions. After hydrogen addition, EtBE degradation stopped. Subsequently, the gas phase was changed back to $\mathrm{N}_{2} / \mathrm{CO}_{2}(80: 20 \mathrm{v} / \mathrm{v})$ and EtBE degradation was re-activated. Placing the culture in an anoxic gas bag did not affect the EtBE degradation rate. However, after placing the culture in a dark water-filled bucket the EtBE degradation ceased.

Ethanol and TBA were detected in the culture liquid. Concentrations fluctuated over time between 8.3 to $51.3 \mu \mathrm{M}$ and 11.7 to $215.7 \mu \mathrm{M}$, respectively. Morphological features observed by microscopy suggested that the liquid in the fed-batch reactor contained algae belonging to the genus Chlorella (Figure S4.3). A microcosm with liquid from the fedbatch reactor exposed to daylight (day/night cycle), amended with $100 \mu \mathrm{g} / \mathrm{ml}$ cycloheximide and a headspace oxygen concentration of about 5\%, degraded EtBE at a similar rate as before the addition of cycloheximide $(4.0 \pm 0.5 \mu \mathrm{M} /$ day). In contrast, the addition of 5 $\mathrm{mM}$ metyrapone interrupted the degradation. In a microcosm $(10 \mathrm{ml})$, containing PBS without metyrapone, the microbial community continuously degraded EtBE. Anoxic liquid from the fed-batch reactor, incubated in the dark at $20^{\circ} \mathrm{C}$ did not show any degradation after 41 days. In a $0.1 \% \mathrm{v} / \mathrm{v}$ transfer of the fed-batch reactor in BG-11 broth and exposed to 


\section{Chapter 4.}

daylight (day/night cycle), algal growth was observed (green deposit), but no ethanol was detected after seven days $(<4.5 \mu \mathrm{M})$.

\section{EtBE degradation pathway}

To elucidate the EtBE degradation pathway we added $50 \mu \mathrm{M}{ }^{13} \mathrm{C}_{6}$ labelled EtBE as carbon source to the microbial community in the fed batch reactor. ${ }^{13} \mathrm{C}$ labelled $\mathrm{NaHCO}_{3}$ increased over time (Figure 4.2). At day 1, ${ }^{13} \mathrm{C}$ labelled TBA was below the detection limit of the NMR, but $49 \mu \mathrm{M}$ TBA was measured by GC-FID equipped with an SPME. From day 1 , the ${ }^{13} \mathrm{C}$ labelled EtBE/TBA ratio increased up to a value of 2.2 for the second ${ }^{13} \mathrm{C}_{6} \mathrm{EtBE}$ dosage (29 days) measured by NMR (Figure 4.2). After a third dosage of ${ }^{13} \mathrm{C}_{6}$ EtBE (48 days), TBA was detected just above the base line of the NMR spectrum.

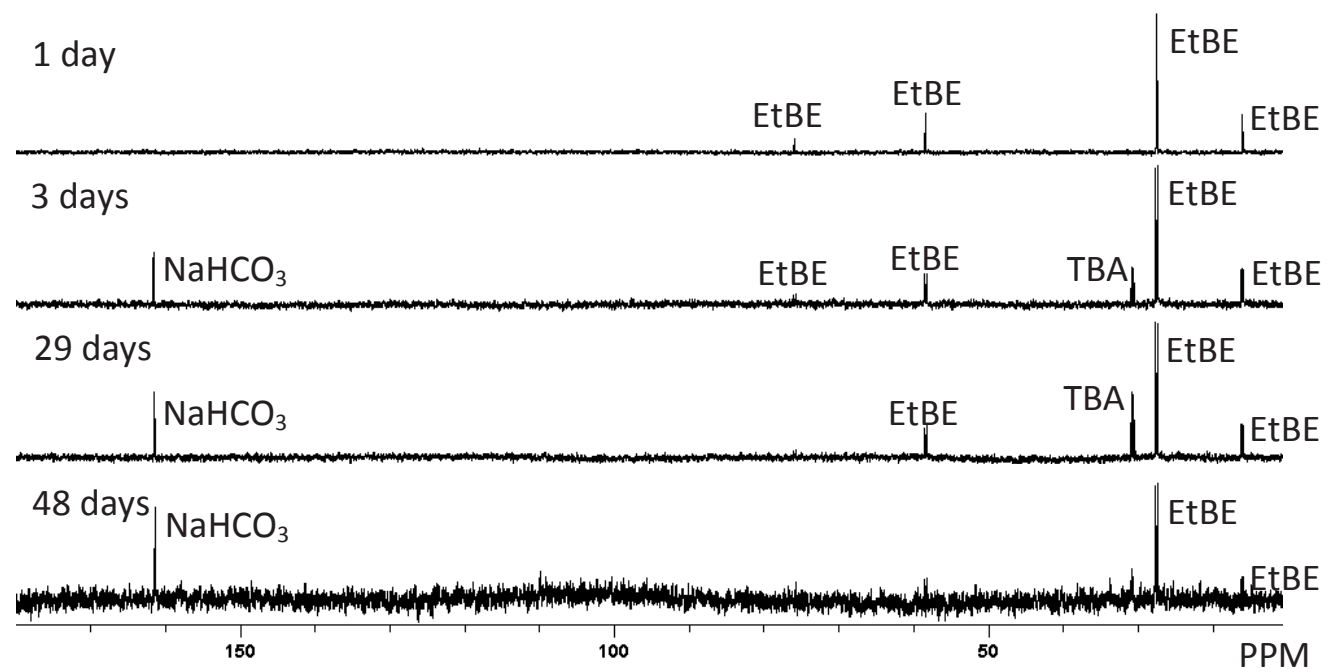

Figure 4.2 Detection of EtBE metabolites via ${ }^{13} \mathrm{C}$ - NMR spectra over time.

\section{Microbial community}

The functional gene $m d p A$ coding for MtBE monooxygenase was not detected in the fedbatch culture $(<1.4$ gene copies/ml sample). The ethB gene encoding cytochrome P450 monooxygenase subunit was also not detected in the microbial community DNA in the fedbatch reactor. The functional gene icmA coding for isobutyryl-CoA mutase was detected at relatively high copy numbers in fed-bath reactor samples $\left(20.0 \pm 5.7 \times 10^{4}\right.$ gene copies $\left./ \mathrm{ml}\right)$ as compared with the total bacterial $16 \mathrm{~S}$ rRNA genes $\left(7.5 \pm 2.1 \times 10^{4}\right.$ gene copies/ml) (Figure 
4.3). Total archaeal $16 \mathrm{~S}$ rRNA genes in the fed-batch reactor were observed at an average of $1.4 \pm 0.2 \times 10^{4}$ gene copies $/ \mathrm{ml}$ sample.

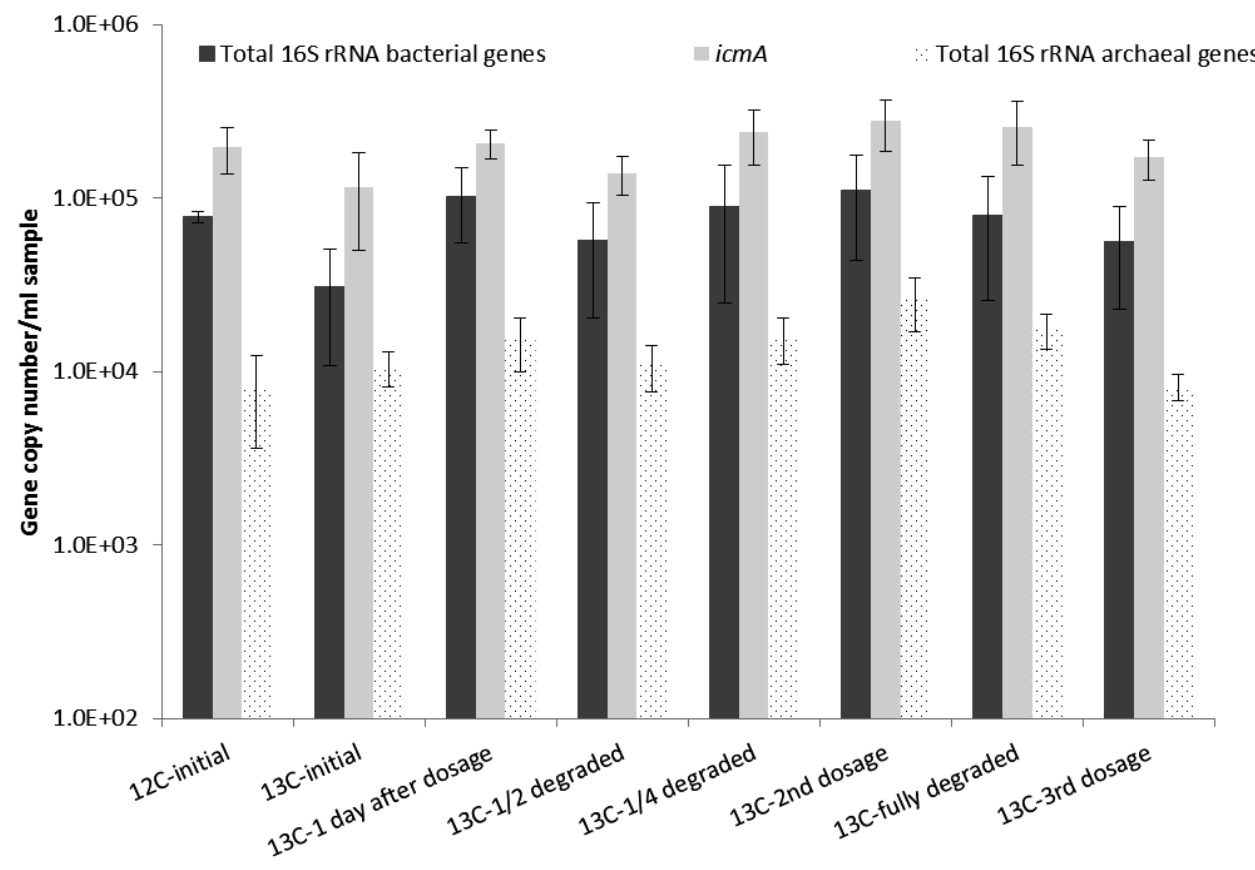

Figure 4.3 qPCR analyses of 16S rRNA genes of total bacteria, icmA genes and 16S rRNA genes of total archaea, at different time points during dosage of ${ }^{13} \mathrm{C}_{6}$ EtBE in the fed-batch reactor. Error bars represent standard deviations based on analytical replicates of the same fed-batch reactor sample.

MiSeq sequencing of PCR-amplified V1-V2 fragments of bacterial 16S rRNA genes was used for description of overall bacterial community composition (Table S4.1). Samples were taken at different time points in the EtBE degradation process (day/night cycle). The phylogenetic diversity (PD whole tree) was similar for all samples with an average of $23 \pm 1.4$, and the number of observed OTUs was 203.4 \pm 12.6 (Table S4.1). MiSeq community analysis did not show a significant difference in relative abundance of the families in the fed-batch reactor between the ${ }^{12} \mathrm{C}$ - and ${ }^{13} \mathrm{C}$-enriched fractions over time (Figure 4.4). Predominant families in the EtBE degrading fed-batch reactor included (in relative abundance): Halomonadaceae (2.4 $\pm 2.6 \%)$, Shewanellaceae (2.1 $\pm 2.1 \%)$, Rhodocyclaceae $(3.4 \pm 1.7 \%)$,

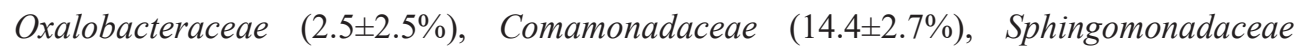
(1.1 $\pm 1.1 \%)$, Hyphomicrobiaceae (3.7 $\pm 0.7 \%)$, Candidatus Moranbacteria (10.4 $\pm 2.7 \%)$, 
Chapter 4.

Candidatus Omnitrophica (2.4 $\pm 0.5 \%)$, Anaerolineaceae $(3.3 \pm 0.8 \%)$, Nocardiaceae $(7.1 \pm 1.7 \%)$ and Blastocatellaceae $(3.5 \pm 0.6 \%)$. 
EtBE degradation by an algal-bacterial culture

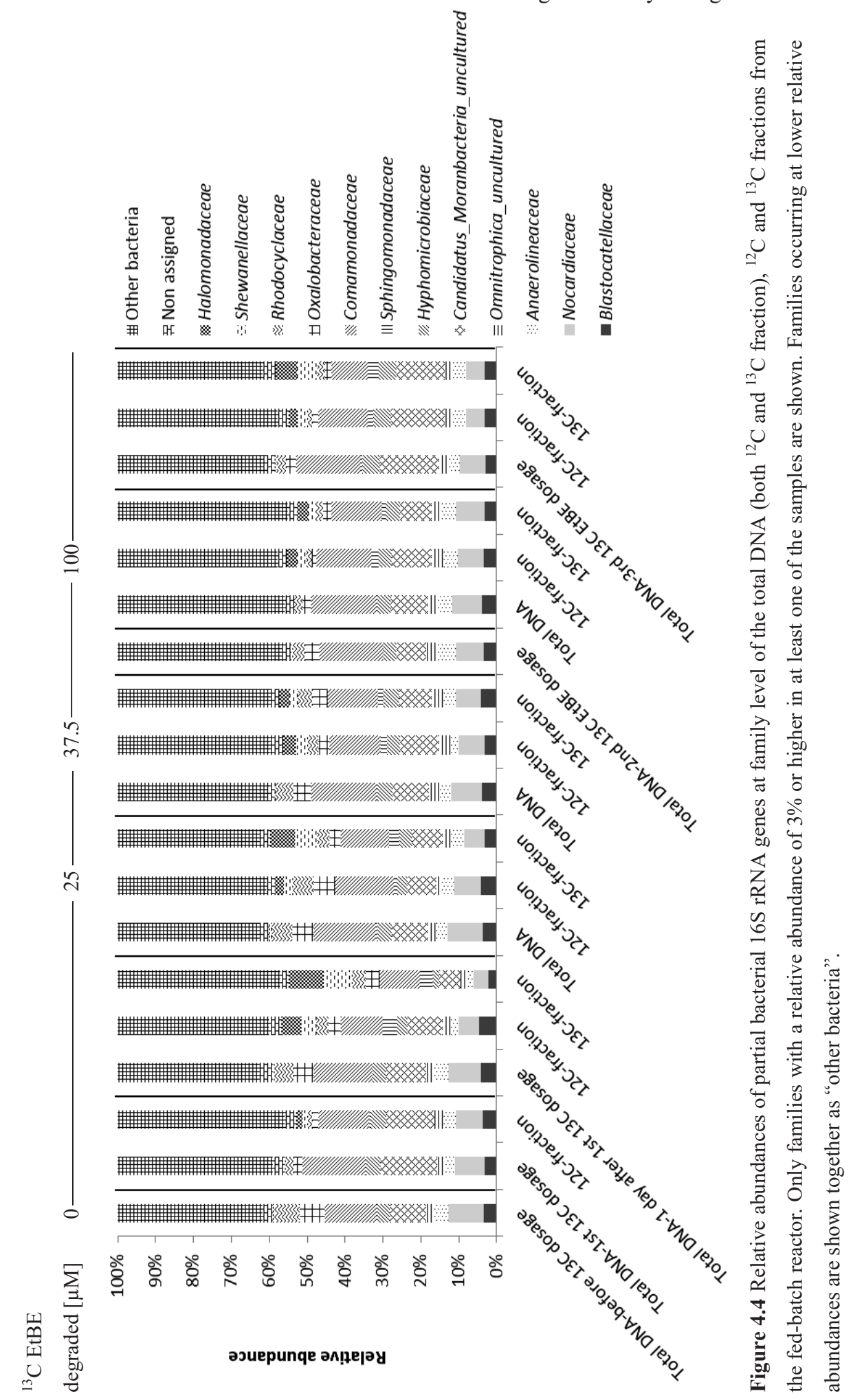




\section{Chapter 4.}

\section{$\underline{\text { Discussion }}$}

The goals of this study were: (i) to determine the EtBE degradation capacity, (ii) to resolve the EtBE degradation pathway and (iii) to characterise the phylogenetic composition of the microbial community involved in EtBE degradation in contaminated groundwater.

In this study it was found that algae play an important role in the EtBE loss. We therefore hypothesize that the oxygen produced by the algae was used by bacteria to degrade the EtBE aerobically. The presence of algae in the monitoring well of this study might be the result of (i) percolating rainwater, (ii) surface water recharge from the nearby stream, (iii) contamination by recirculated drilling fluids when the monitoring well was installed, or (iv) growth of algae in the groundwater $[195,208]$. A previous study observed a diverse microeukaryotic community at a depth of 1 to $5 \mathrm{~m}$ below the surface in an aquifer polluted with landfill leachate [208]. Furthermore, viable algae were detected down to $225 \mathrm{~m}$ in the subsurface as described elsewhere [195].

\section{EtBE degradation in microcosms under different redox conditions}

The microcosms with gasoline contaminated groundwater without any addition of electron acceptor or medium, and the microcosms containing groundwater at different redox conditions did not show EtBE degradation in the dark. The aerobic microbial community in this groundwater degraded the EtBE $(33.6 \pm 2.4 \mu \mathrm{M} /$ day) in the lower range of degradation rates as reported previously under aerobic conditions (35 to $2208 \mu \mathrm{M} \mathrm{day}^{-1}$ ) [12, 188, 209, 210]. The higher aerobic EtBE degradation rates determined in previous studies might be due to the fact that those experiments were done either with pure cultures or with enrichments obtained with EtBE at relatively high oxygen concentrations. The microbial community described in our study was obtained from anoxic groundwater containing a mixture of contaminants including EtBE, and enriched at oxygen concentrations lower than $0.1 \%$.

The microcosm without electron acceptor and medium addition containing up to $50 \%$ oxygen, produced by algae in a day/night light cycle, degraded EtBE at a lower rate $(0.8 \pm 0.1 \mu \mathrm{M} /$ day) compared with these previously reported rates. This might be due to the fact that this microcosm had been incubated without oxygen in the dark for 872 days before exposure to light to induce oxygen production by the algae Scenedesmus and/or Chlorella. In this microcosm containing groundwater without electron acceptor and medium, TBA 
was not accumulating over time, indicating further degradation of TBA after exposure to light [211].

\section{EtBE degradation in a fed-batch reactor}

The EtBE degradation rates in the fed-batch reactor from $1.0 \pm 0.2 \mu \mathrm{M}_{\text {.day }}{ }^{-1}$ to $6.7 \pm 1.6$ $\mu \mathrm{M}$.day ${ }^{-1}$ are lower than those found in aerobic cultures in microcosms. The lower EtBE degradation rates found in our study could be explained by the fact that the fed-batch reactor in this study was micro-oxic $\left(<0.1 \% \mathrm{O}_{2}\right)$ resulting in oxygen limited growth instead of having a sufficiently high oxygen concentration for exponential growth. When the fedbatch reactor was continuously exposed to light (4125 lux), the degradation rate was $6.8 \pm 1.5 \mu \mathrm{M}$.day ${ }^{-1}$, and the oxygen concentration increased to a value of up to $10 \%$. No EtBE degradation was observed under anoxic conditions in the dark. This result is in line with the hypothesis that microalgae in the groundwater produce oxygen when exposed to light and that this oxygen is subsequently used by the bacteria to degrade EtBE. In addition, the cytochrome P450 inhibitor, metyrapone, interrupted EtBE degradation. This result is in line with previous studies suggesting that a cytochrome P450 is involved in the oxidative cleavage of the ether bond [190, 193]. Furthermore, the addition of $100 \mu \mathrm{g} / \mathrm{ml}$ cycloheximide in the presence of oxygen did not have an effect on the EtBE degradation rate, suggesting that the eukaryotes (microalgae) in the groundwater did not degrade the EtBE. The eth $B$ gene encoding the cytochrome P450 monooxygenase subunit was not detected in the EtBE degrading microbial community. This suggests that other (mono)oxygenase enzymes sensitive to metyrapone were active in this community, which were not detected by the ethB assay developed by Schuster and co-workers [193]. For MtBE degradation it was found that a symbiotic algal-bacterial system could increase the MtBE degradation rate due to higher dissolved oxygen concentrations as a result of photosynthesis [196].

John and co-workers found that certain species of algae can produce ethanol during darkanaerobic fermentation [212]. No ethanol was measured in the dark anaerobic microcosm with BG-11 broth, suggesting that the algae Chlorella and Scenedesmus in this study did not produce ethanol. Therefore, the measured ethanol in the fed-batch reactor is most likely a metabolite in the EtBE degradation pathway. The ${ }^{13} \mathrm{C}$ TBA which was not detected after $24 \mathrm{~h}$ by NMR was detected at $49 \mu \mathrm{M}$ using GC-FID equipped with an SPME fiber. This suggests that the ${ }^{13} \mathrm{C}$ labelled TBA concentrations were too low to be detected by NMR. 


\section{Chapter 4.}

After $24 \mathrm{~h},{ }^{13} \mathrm{C}$ TBA concentrations increased relatively compared with declining ${ }^{13} \mathrm{C} \mathrm{EtBE}$ concentrations, indicating that TBA is indeed a metabolite from EtBE degradation. This is in line with previous reports suggesting that TBA is the main metabolite of EtBE degradation [209, 210]. Although ${ }^{13} \mathrm{CO}_{2}$ was detected by NMR, we could not unequivocally conclude whether complete EtBE degradation up to $\mathrm{CO}_{2}$ was occurring.

The high number of the $i c m A$ gene $\left(20.0 \pm 5.7 \times 10^{4}\right.$ gene copies $/ \mathrm{ml}$ sample) compared to total bacterial $16 \mathrm{~S}$ rRNA gene copies $\left(7.5 \pm 2.1 \times 10^{4}\right.$ gene copies $/ \mathrm{ml}$ sample) may indicate that there are multiple icmA gene copies in the DNA of bacteria or that the primers amplify other genes with high similarity. Furthermore, this relatively high amount of icmA genes suggests that EtBE was degraded via TBA and 2-hydroxybuyrate according to the metabolic pathway involving isobutyryl-CoA mutase as was suggested previously [173]. While being beyond the scope of the current study, future experiments should include analysis of the icmA gene at the RNA level to confirm that this gene is expressed during growth on EtBE. Total archaeal 16S rRNA genes were detected at high concentrations of $1.4 \pm 0.2 \times 10^{4}$ gene copies/ml sample in the fed-batch reactor, however, their role in the EtBE degradation is not known.

Analysis of 16S rRNA gene amplicon sequencing profiles revealed a similar phylogenetic diversity and OTU number for the pooled ${ }^{12} \mathrm{C}$ - and ${ }^{13} \mathrm{C}$-enriched fractions. The analysis indicated that total and active members of the communities represented by the light and heavy DNA fractions, respectively, were very similar to each other. This similarity between labelled and unlabelled nucleic acids is in agreement with previous studies [213, 214]. These studies found that while metabolically inactive bacteria or extracellular DNA was present, the similarity between labelled and unlabelled DNA indicated that most recovered OTUs had metabolically active representatives in this community. It may also be that the ${ }^{13} \mathrm{C}$ EtBE concentrations used in this study were not sufficient to measure a change in phylogenetic diversity in the 'heavy' carbon fraction. Relatively high numbers of Comamonadaceae and Candidatus Moranbacteria were detected in the fed-batch reactor. This result is in line with a previous study, were a strain of Comamonas testosteroni was isolated with the ability to degrade EtBE as the sole source of carbon and energy [189]. 


\section{Conclusion}

This is the first study describing degradation of EtBE in an algal-bacterial culture. In this culture, algae (i.e. Chlorella or Scenedesmus) use light to produce oxygen, which is consumed by the bacterial community to aerobically degrade EtBE. The algal-bacterial interaction in this study has advantages compared with conventional aerobic treatments. Firstly, this interaction represents a closed system without the need for aeration. Therefore, the risk of EtBE evaporation is minimized. Secondly, there is no need for expensive, external oxygen supply [196]. More research aiming at a better understanding and control of the EtBE degradation rates, pathway and the responsible microbes is needed to explore the possibilities to implement such algal-bacterial consortia in for example groundwater or wastewater treatment. 


\section{Chapter 4.}

\section{Supplementary Figures and Tables}

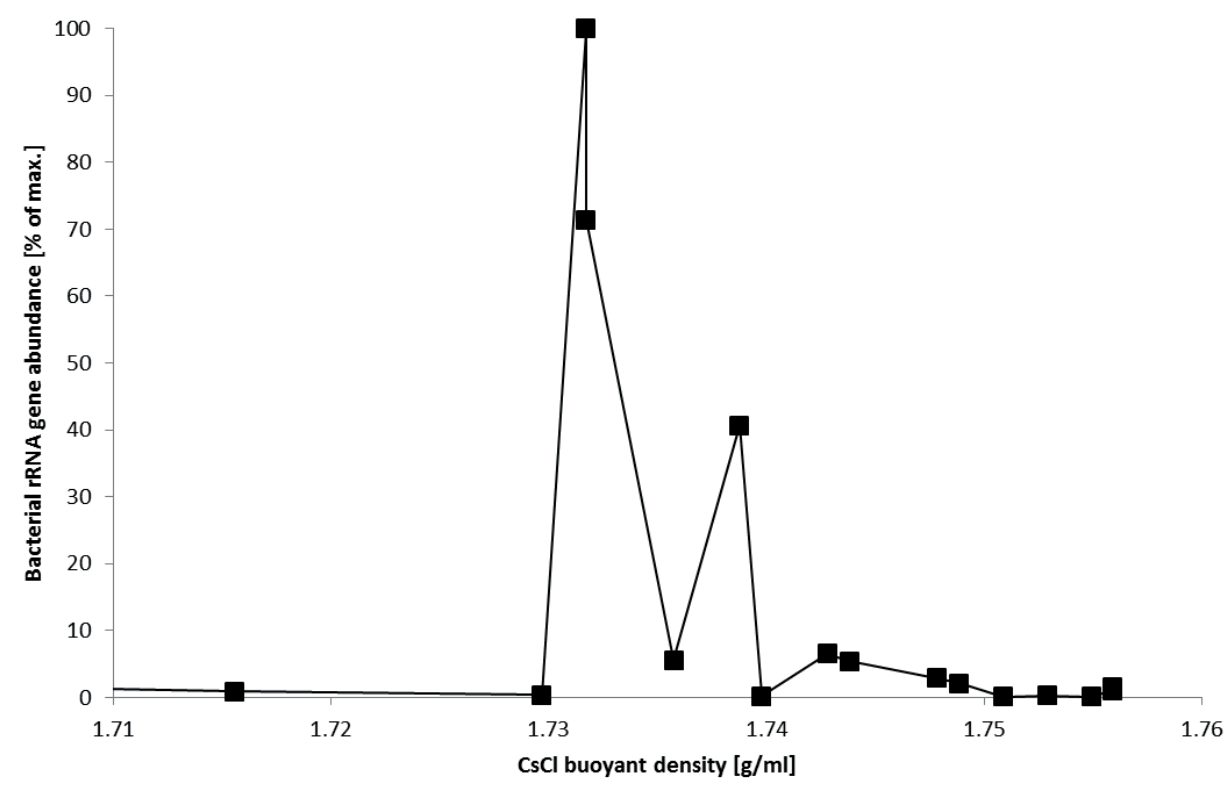

Figure S4.1 Density gradient of "heavy" and "light" fraction after three ${ }^{13} \mathrm{C}$ EtBE dosages.

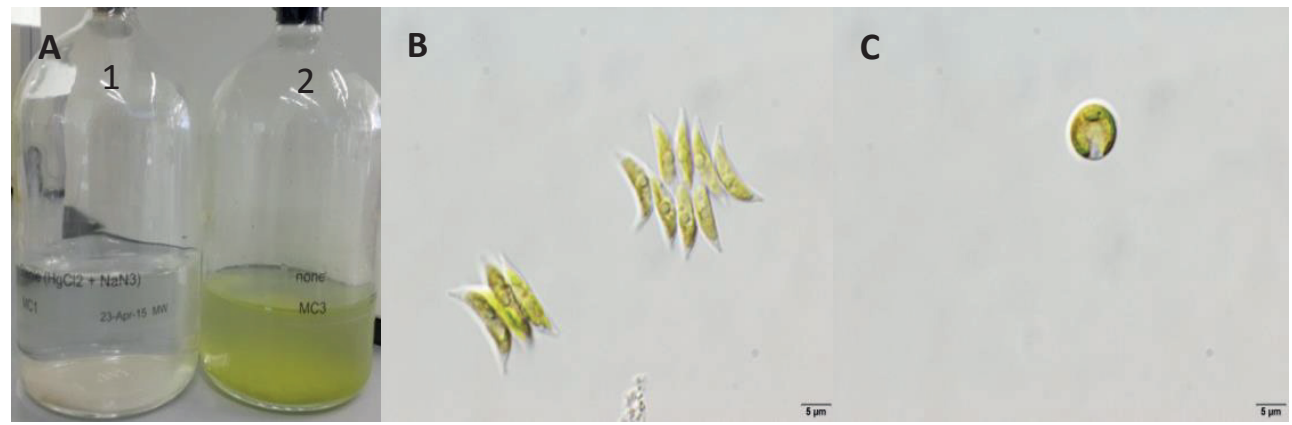

Figure S4.2 Algal growth in a microcosm. (A) Microcosms after eight weeks in a day-night cycle. Microcosm 1 is an abiotic control microcosm, which was sterilized by autoclaving and the addition of $100 \mathrm{mg} / 1 \mathrm{HgCl} \mathrm{H}_{2}$ and $2 \mathrm{mg} / \mathrm{NaN}_{3}$. Microcosm 2 is an unamended control microcosm with algae tentatively identified as Chlorella and Scenedesmus. (B) and (C) are microscope images of the tentatively identified Scenedesmus and Chlorella, respectively, in the unamended control microcosm. 
Figure S4.3 Algal cell tentatively identified as Chlorella in the liquid of the fed-batch reactor.

Table S4.1 MiSeq sample characteristics.

\begin{tabular}{|c|c|c|c|}
\hline Sample & $\begin{array}{l}\text { Sequence reads } \\
\text { per sample }\end{array}$ & OTUs & Phylogenetic diversity \\
\hline $\begin{array}{l}\text { Total DNA-before } \\
\text { 13C dosage }\end{array}$ & 175,944 & 199 & 22.6 \\
\hline $\begin{array}{l}\text { Total DNA-after } 13 \mathrm{C} \\
\text { dosage }\end{array}$ & 292,359 & 192 & 21.8 \\
\hline $12 \mathrm{C}$-fraction & 87,965 & 213 & 24.0 \\
\hline $\begin{array}{l}\text { Total DNA-1 day after } \\
\text { dosage }\end{array}$ & 212,614 & 184 & 21.6 \\
\hline $12 \mathrm{C}$-fraction & 24,918 & 214 & 23.8 \\
\hline $13 \mathrm{C}$-fraction & 21,999 & 224 & 26.2 \\
\hline $\begin{array}{l}\text { Total DNA-half of } \\
13 \mathrm{C} \\
\text { EtBE degraded }\end{array}$ & 161,181 & 192 & 22.1 \\
\hline $12 \mathrm{C}$-fraction & 81,247 & 195 & 21.9 \\
\hline 13C-fraction & 21,041 & 211 & 24.6 \\
\hline $\begin{array}{l}\text { Total DNA- } \\
\text { threequarters of } 13 \mathrm{C} \\
\text { EtBE degraded }\end{array}$ & 225,750 & 190 & 21.0 \\
\hline $12 \mathrm{C}$-fraction & 42,574 & 203 & 23.1 \\
\hline $13 \mathrm{C}$-fraction & 31,966 & 207 & 22.4 \\
\hline $\begin{array}{l}\text { Total DNA-2 } 2^{\text {nd }} 13 \mathrm{C} \\
\text { EtBE dosage }\end{array}$ & 144,848 & 196 & 22.0 \\
\hline $\begin{array}{l}\text { Total DNA-13C EtBE } \\
\text { fully degraded }\end{array}$ & 222,819 & 198 & 22.7 \\
\hline $12 \mathrm{C}$-fraction & 47,108 & 203 & 23.1 \\
\hline 13C-fraction & 70,980 & 222 & 24.4 \\
\hline
\end{tabular}


Chapter 4.

Total DNA-3 ${ }^{\text {rd }} 13 \mathrm{C}$ EtBE dosage

143,611

187

21.1

12C-fraction

33,151

208

22.9

13C-fraction

43,257

226

25.2 
EtBE degradation by an algal-bacterial culture 
Chapter 4.

108 
Chapter 5. Isolation of anaerobic benzene degrading bacteria and assessment of bioaugmentation potential

Marcelle J. van der Waals, Melike Balk, Hauke Smidt and Jan Gerritse

Manuscript in preparation 


\section{Chapter 5.}

\section{Abstract}

Benzene is a constituent of crude oil and gasoline and poses a toxicological risk after release into the environment. In this study we evaluated the bioaugmentation potential of a continuous culture, maintained for more than 15 years on benzene with nitrate as electron acceptor. Analysis of different inoculation materials obtained from the continuous culture revealed that transfers with biofilm material yielded first order benzene degradation rates of up to $11.2 \pm 8.8 \times 10^{-3}$ day $^{-1}$. The use of more mature brown biofilm characterized by high protein concentrations and a different microbial community composition when compared with younger white biofilm resulted in higher benzene degradation rates in microcosms. Several microorganisms were isolated from the continuous culture, including strains most closely related to Bacillus cereus, Ochrobactrum anthropi, Comamonas badia and Mesorhizobium plurifarium. Three isolates namely Bacillus sp. strain BOC1, Comamonas sp. strain BOC3 and Ochrobactrum sp. strain BOC2, were all able to grow on benzene with nitrate as electron acceptor. The capacity to grow on benzene with nitrate was lost in microcosm cultures maintained on acetate or pyruvate in the absence of benzene. A pure culture of Pseudomonas sp. strain BN, which had lost the capacity to grow anaerobically on benzene, was exposed to extracellular DNA extracted from the continuous culture. Restoration of anaerobic benzene degradation capacity suggested that the benzene carboxylase encoding gene, $a b c A$, might be taken up, further supported by the fact that strain $\mathrm{BN}$ is predicted to possess type IV pili based on its genome sequence.

\section{Introduction}

Benzene is a constituent of crude oil and gasoline and is often considered as riskdetermining compound at contaminated locations, because it is toxic and easily dissolves and migrates in groundwater [11]. Anaerobic degradation of benzene in oxygen depleted contaminated subsurface environments can reduce its toxicological risk and distribution after accidental release in the environment. We studied an anaerobic benzene-degrading, denitrifying microbial consortium which has been enriched and maintained in a continuous culture for more than 15 years [43]. Detection of the benzene carboxylase gene, $a b c A$, in the metagenome of this microbial consortium, transcription of the $a b c A$ and $a b c D$ genes and the detection of benzoic acid, indicates that benzene is degraded through an initial carboxylation step [57, 215]. 
Several biological, physical and chemical technologies can be applied for site specific degradation of contaminants in situ or ex situ [216]. In situ enhanced bioremediation techniques are performed in the contaminated aquifer while ex situ bioremediation takes place above-ground. Generally, ex situ techniques are more expensive compared with in situ techniques because of excavation costs [91]. If time is not the critical factor, in situ clean-up enhanced biodegradation is potentially an efficient remediation method. In situ bioremediation (i.e. natural attenuation or enhanced bioremediation) has been successfully applied for the degradation of many groundwater contaminants such as chlorinated solvents, gasoline methyl tert-butyl ether and other fuel constituents or petroleum hydrocarbons as reviewed by Farhadian and co-workers [90]. Enhancing the indigenous microbial community by addition of electron acceptors, electron donors or nutrients is known as biostimulation [94]. If the native microbial community at the contaminated site lacks a significant number of microorganisms capable of biodegradation of the contaminants, exogenously grown organisms can be added. This process is referred to bioaugmentation [97]. Before performing bioaugmentation field tests, it is important to determine the potential effectiveness of different bioaugmentation strategies in laboratory experiments using appropriate biological and chemical controls.

In this study, we used an anaerobic benzene-degrading continuous culture, which has been enriched with nitrate as electron acceptor for more than 15 years [57]. Both, the microbial biofilm formed on the glass and metal surfaces and the liquid phase in the reactor vessel were used as inoculation material for laboratory bioaugmentation tests.

Anaerobic benzene degradation has been demonstrated in enrichments [39-44, 61] and pure cultures [45]. Dechloromonas strains RCB and JJ [46], two Azoarcus strains (DN11 and AN9) [44], Bacillus cereus [45] and Pseudomonas stutzeri strain BN [47] have been isolated for their ability to degrade benzene with nitrate as electron acceptor. Pseudomonas stutzeri strain BN was previously isolated from the continuous culture described in this study [47]. The genome of Dechloromonas strain RCB does neither include the genes encoding benzylsuccinate synthase nor enzymes involved in the benzoyl-CoA pathway for mono-aromatics [113]. This and potentially other isolates might therefore degrade benzene through intra-aerobic pathways that use oxygen released e.g. from nitrite or chlorite dismutation. To the best of our knowledge, there has been no report proving the isolation of strictly anaerobic denitrifying benzene degraders. Bacteria related to the anaerobic Gram- 


\section{Chapter 5.}

positive Peptococcaceae have been shown to play an important role in anaerobic benzene degradation under nitrate reducing $[43,57,114,215]$, iron-reducing $[50,115]$ and sulphatereducing conditions $[43,69,70,78,116]$. It was also suggested that benzene-degrading species (e.g. Peptococcaceae) coexist in a syntrophic reaction with benzoate degrading organisms such as Azoarcus [43, 114].

Based on metagenome and metatranscriptome analyses of biomass retrieved from the above-mentioned continuous culture, it was previously suggested that there were genes encoding phage-related proteins and transposable elements on the same contig as the $a b c A$ gene [215]. Therefore, the overall goal of this study was to determine the transferability of anaerobic benzene degradation in a microbial consortium and how this can be used for bioaugmentation of microbial communities in an oil-tar contaminated aquifer.

The approach that was followed to reach this goal included: (i) to determine the best inoculation material (e.g. biofilm, liquid with dispersed cells) for a pilot test, (ii) to isolate and identify anaerobic denitrifying benzene degraders, and (iii) to determine transferability of $a b c A$ genes encoding benzene carboxylase.

\section{Materials and Methods}

The anaerobic continuous culture used in this study was operated for more than 15 years on benzene as electron donor under nitrate-reducing conditions as described previously [43, 57]. Briefly, the medium contained salts, trace elements and vitamins and had a $\mathrm{pH}$ of 6.7 . The temperature in the culture vessel was maintained at $25^{\circ} \mathrm{C}$ and the liquid phase was stirred at $100 \mathrm{rpm}$. The headspace of the reactor vessel was flushed with $80 \% / 20 \%(\mathrm{v} / \mathrm{v})$ $\mathrm{N}_{2} / \mathrm{CO}_{2}$, and $100 \mu \mathrm{M}$ benzene was injected from a $22 \mathrm{mM}$ anoxic aqueous stock solution directly into the liquid phase. The $\mathrm{N}_{2} / \mathrm{CO}_{2}$ gas was passed over a $460 \mathrm{ml}$ glass column filled with hot copper filings $\left(250^{\circ} \mathrm{C}\right)$ to remove traces of oxygen before entering the growth vessel. The liquid dilution rate of the reactor was 0.25 day $^{-1}$.

\section{Microcosm experiments using liquid from the continuous culture}

Initially, microcosms were incubated with (i) both medium $(50 \mathrm{ml})$ and liquid from the continuous culture $(50 \mathrm{ml})$, and (ii) exclusively liquid from the continuous culture $(100 \mathrm{ml})$. These microcosms were prepared by injecting the continuous culture liquid or medium, respectively using a $60 \mathrm{ml}$ syringe and water lock in $80 / 20 \% \mathrm{~N}_{2} / \mathrm{CO}_{2}(\mathrm{v} / \mathrm{v})$ flushed $250 \mathrm{ml}$ 
serum bottles (Glasgerätebau Ochs GmbH, Bovenden, Germany) crimp-sealed with viton rubber stoppers (Rubber BV, Hilversum, the Netherlands) and aluminium crimp caps (Grace Maryland, USA). Continuous culture samples with biofilm aggregates were obtained as described previously [57]. When benzene in the microcosms was depleted, it was re-added to $100 \mu \mathrm{M}$ from a $22 \mathrm{mM}$ anoxic stock solution.

\section{Bioaugmentation experiments using groundwater from a tar contaminated site}

In this experiment anoxic groundwater extracted from the vicinity of a tar plume at a contaminated site of a former pintsch gas factory near a railway station in the Netherlands was used to determine the microbial degradation potential for benzene. At the tar-impacted area the redox conditions varied from nitrate-, sulphate- and manganese-reducing to ironreducing conditions and the $\mathrm{pH}$ was around 6 (Table S5.1). Groundwater for the microcosms was collected from a monitoring well located upstream of the tar plume, in gas tight 201 polypropylene bottles (Nalgene, NY, USA). The bottles, filled completely with groundwater to prevent the introduction of oxygen, were transported the same day to the laboratory, and stored at $4{ }^{\circ} \mathrm{C}$ for preparation of microcosms within seven days after sampling. Microcosms were prepared under an $95 \% / 5 \% \mathrm{~N}_{2} / \mathrm{CO}_{2}(\mathrm{v} / \mathrm{v})$ flow in $250 \mathrm{ml}$ serum bottles (Glasgerätebau Ochs $\mathrm{GmbH}$, Bovenden, Germany) and crimp-sealed with viton rubber stoppers (Rubber BV) and aluminium crimp caps (Grace). Traces of $\mathrm{O}_{2}$ were stripped from the $\mathrm{N}_{2} / \mathrm{CO}_{2}$ gas as described previously [57]. The microcosms contained at least $90 \mathrm{ml}$ of groundwater without any contaminants and were amended with $100 \mu \mathrm{M}$ benzene added from a $22 \mathrm{mM}$ anoxic stock solution. When benzene was depleted, it was readded to $100 \mu \mathrm{M}$ from the same stock solution. All microcosms were amended with $4 \mathrm{mM}$ nitrate from an anoxic, autoclaved stock solution. In addition, medium control microcosms contained sterile medium as described previously [57]. The $\mathrm{pH}$ of the medium with groundwater was 7.0. The microcosms were incubated upside down and continuously shaken at $100 \mathrm{rpm}$ (Certomat, B|Braun, Melsungen, Germany) in the dark at $20^{\circ} \mathrm{C}$. Abiotic control microcosms were autoclaved and contained $100 \mathrm{mg} / 1 \mathrm{HgCl}_{2}$ plus $2 \mathrm{mg} / 1 \mathrm{NaN}_{3}$. A detailed description of the prepared microcosms with the respective inocula is presented in Table 5.1. All microcosms were prepared in duplicate. 


\section{Chapter 5.}

Table 5.1 Description of the bioaugmentation microcosms using groundwater from a tar contaminated site.

\begin{tabular}{|c|c|c|c|}
\hline Condition & Addition & Inoculum & $\begin{array}{c}\text { Groundwater } \\
{[\mathrm{ml}]}\end{array}$ \\
\hline Sterile groundwater & $\begin{array}{c}\text { Autoclaved }+100 \mathrm{mg} / 1 \mathrm{HgCl}_{2} \\
+2 \mathrm{mg} / 1 \mathrm{NaN}_{3}\end{array}$ & None & 100 \\
\hline $\begin{array}{l}\text { Sterile groundwa- } \\
\text { ter+inoculum }\end{array}$ & $\begin{array}{l}\text { Autoclaved }+100 \mathrm{mg} / 1 \mathrm{HgCl}_{2} \\
+2 \mathrm{mg} / 1 \mathrm{NaN}_{3}\end{array}$ & $\begin{array}{l}\text { Filtered } \\
\text { cells }\end{array}$ & 100 \\
\hline $\begin{array}{l}\text { Autoclaved groundwa- } \\
\text { ter+living inoculum }\end{array}$ & 2x Autoclaved-groundwater & $\begin{array}{l}\text { Filtered } \\
\text { cells }\end{array}$ & 100 \\
\hline Retentostat liquid & None & $\begin{array}{l}10 \mathrm{ml} \text { reten- } \\
\text { tostat liquid }\end{array}$ & 90 \\
\hline $\begin{array}{l}\text { Continuous culture } \\
\text { liquid }\end{array}$ & None & $\begin{array}{l}10 \mathrm{ml} \text { con- } \\
\text { tinuous } \\
\text { culture liq- } \\
\text { uid }\end{array}$ & 90 \\
\hline Medium & $10 \mathrm{ml}$ medium & None & 90 \\
\hline Medium+filtered cells & $10 \mathrm{ml}$ medium & $\begin{array}{l}\text { Filtered } \\
\text { cells }\end{array}$ & 90 \\
\hline $\begin{array}{l}\text { Living inoculum using } \\
\text { filtered cells }\end{array}$ & None & $\begin{array}{l}\text { Filtered } \\
\text { cells }\end{array}$ & 100 \\
\hline $\begin{array}{l}\text { Continuous culture } \\
\text { biofilm }\end{array}$ & None & $\begin{array}{l}10 \mathrm{ml} \text { liquid } \\
\text { with biofilm }\end{array}$ & 90 \\
\hline
\end{tabular}

Microcosms containing filtered cells were prepared by filtration of $10 \mathrm{ml}$ continuous culture liquid over a $0.2 \mu \mathrm{m}$ polycarbonate filter (Merck Millipore). The filter containing microbial cells was added to the microcosm under a constant $80 / 20 \%(\mathrm{v} / \mathrm{v}) \mathrm{N}_{2} / \mathrm{CO}_{2}$ flow and rigorously shaken for 10 seconds by hand. Subsequently, the filter was removed under a constant $80 / 20 \%(\mathrm{v} / \mathrm{v}) \mathrm{N}_{2} / \mathrm{CO}_{2}$ flow and the microcosm was re-capped with a viton rubber stopper and aluminium crimp cap. Liquid from the reactor vessel was taken directly from the liquid phase of the culture vessel using a $60 \mathrm{ml}$ syringe (Henke-Sass Wolf, Tuttlingen, Germany) via a sampling outlet and viton tubing (Rubber BV). Biomass from a retentostat system was taken in an attempt to have an increased amount of benzene degrading cells as was previously described [57]. Liquid from the retentostat was taken directly from the liquid phase of the retentostat batch using a $10 \mathrm{ml}$ syringe (Romed, Wilnis, the Netherlands) and 0.6x25 mm needle (BD microlance, Eysins, Switzerland) [57]. The microcosms containing continuous culture biofilm were prepared by scraping off $5 \mathrm{~cm}^{2}$ fresh white biofilm and $4 \mathrm{~cm}^{2}$ mature brown biofilm as previously described [57]. 


\section{Enrichment and isolation of benzene-degrading strains from the continuous culture}

To isolate benzene-degrading microorganisms, biofilm samples were taken from the reactor vessel as previously described [43]. Two samples $(50 \mathrm{ml})$ were inoculated in $80 \% / 20 \%$ $\mathrm{N}_{2} / \mathrm{CO}_{2}(\mathrm{v} / \mathrm{v})$ flushed $117 \mathrm{ml}$ serum microcosms, after which $50 \mu \mathrm{M}$ benzene was added from a $24 \mathrm{mM}$ anoxic, autoclaved stock solution.

Concentrated stock solutions of substrates were prepared anoxically, under a $100 \%$ $\mathrm{N}_{2}$ atmosphere, sterilized by filtration $(0.2 \mu \mathrm{m}$ pore size filters (Merck Millipore), and added to medium to final concentrations of 5 to $20 \mathrm{mM}$. The medium contained per liter: $1 \mathrm{~g}$

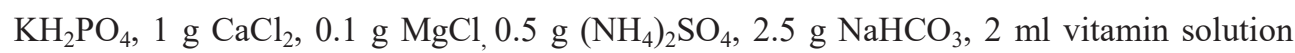
[217] and $1 \mathrm{ml}$ trace element solution (SL-10, www.dsmz.de). Initial cultures were transferred $(1 \% \mathrm{v} / \mathrm{v})$ to microcosms containing pyruvate $(20 \mathrm{mM})$, fumarate $(20 \mathrm{mM})$, crotonate $(20 \mathrm{mM})$ or benzoate $(5 \mathrm{mM})$ in the presence of nitrate $(20 \mathrm{mM})$. All microcosms were incubated at $20^{\circ} \mathrm{C}$ in the dark. Additionally, $20 \mathrm{mM} \mathrm{NH}_{4} \mathrm{Cl}$ and $20 \mathrm{mM} \mathrm{NO}_{3}\left(\mathrm{NaNO}_{3}\right)$ were added to the microcosms.

Serial dilutions of these initial cultures were prepared in liquid medium containing the different substrates as described above. The highest dilution of each culture showing growth was used for further study. Dilution series of the cultures were prepared in agar media (1.8\% [w/v] agar noble, Difco, MI, USA) in serum vials (roll vial technique). Colonies were picked from the highest dilution with growth and inoculated into liquid media. This procedure was repeated several times. Purity of the isolates was confirmed by incubations at $20^{\circ} \mathrm{C}$ under anoxic and oxic conditions in liquid medium containing $10 \mathrm{~g} / 1$ yeast extract (BBL-Becton Dickinson, NJ, USA) or in Wilkins-Chalgren broth (Oxoid, Hampshire, UK).

Cell morphologies and purity for each culture were examined by phase-contrast microscopy (Leica, Wetzlar, Germany).

The ability of strains to metabolise different electron donors and acceptors was tested in the medium as described above. Substrates were added from sterile, anoxic concentrated stock solutions. The following electron and carbon sources were tested in combination with different electron acceptors: glucose $(20 \mathrm{mM})$, 4-hydroxybenzoate $(3 \mathrm{mM})$ with nitrate, benzoate $(3 \mathrm{mM})$ with nitrate, methanol $(5 \mathrm{mM})$, lactate $(20 \mathrm{mM})$ and acetate $(5$ $\mathrm{mM})$ with nitrite $(5 \mathrm{mM})$, chlorate $(5 \mathrm{mM})$, sulphate $(5 \mathrm{mM})$ or perchlorate $(5 \mathrm{mM})$. 


\section{Chapter 5.}

\section{Phylogenetic Analysis}

For the phylogenetic identification of the isolates, total genomic DNA was extracted using the FastDNA SPIN kit for Soil and the FastPrep Instrument (MP Biomedicals) as described by the manufacturer. The $16 \mathrm{~S}$ rRNA genes were amplified with the primer set 27F/1492R for bacteria and using a CFX96 real-time PCR machine (Bio-Rad). Sanger sequencing of amplicons was done at Eurofins MWG (Barneveld, the Netherlands). Sequences were assembled with DNABaser software 3.5.3. The sequences were checked for reading errors with the alignment programs of the ARB package [218]. Partial 16S rRNA gene sequences were compared to sequences stored in the publicly accessible GenBank database for preliminary identification using the NCBI BLAST algorithm [136]. Partial nucleotide sequences determined in this study have been submitted to the EMBL database under the accession numbers KT425062 (Bacillus sp. strain BOC1), KT425063 (Ochrobactrum sp. strain BOC2) and KT425064 (Comamonas sp. strain BOC3). The 16S rRNA gene sequence of Mesorhizobium sp. strain B3 was deposited previously under accession number DQ342274. Pseudomonas sp. strain BN was kindly obtained from Fons Stams and Marjet Oosterkamp (Laboratory of Microbiology, Wageningen University, the Netherlands).

The pure cultures were routinely maintained in batches with medium as described above with the addition of pyruvate (Bacillus sp. strain BOC1), acetate (Ochrobactrum sp. strain BOC2 and Pseudomonas sp. strain BN) or propionate (Comamonas sp. strain BOC3 and Mesorhizobium sp. strain B3), with the addition of $20 \mathrm{mM}$ nitrate $\left(\mathrm{NaNO}_{3}\right)$.

\section{Extracellular $a b c A$ gene experiment}

The stirring rate in the continuous culture was increased from $100 \mathrm{rpm}$ to $500 \mathrm{rpm}$ for 1 hour to disperse biofilm attached at the glass wall in the liquid phase. Samples of $50 \mathrm{ml}$ containing biofilm aggregates were taken directly from the liquid phase as described previously [57]. We assumed that the DNA of the continuous biofilm culture contains the $a b c A$ gene at a concentration of $9.9 \pm 8.1 \times 10^{7}$ gene copies $/ \mathrm{ml}$ sample as was previously described [57]. The DNA in these samples was precipitated with ethanol as described by Darbre [219]. The pelleted DNA was air-dried to dispose of the remaining ethanol. The collected DNA was dissolved in $4 \mathrm{ml}$ of elution buffer (MoBio, CA, USA) and $0.5 \mathrm{ml}$ was dosed to approximately $40 \mathrm{ml}$ of pure cultures of Bacillus sp. strain BOC1, Ochrobactrum sp. strain 
BOC2, Mesorhizobium sp. strain B3, and Pseudomonas sp. strain BN, which had been grown in batches without benzene for four years.

\section{Chemical and molecular analyses}

Headspace samples of $0.5 \mathrm{ml}$ were taken from the microcosms using a $1 \mathrm{ml}$ sterile syringe (B|Braun) and $0.5 \times 25 \mathrm{~mm}$ needle (Henke Sass Wolf), and benzene was measured on a Varian 3800 GC-FID as previously described [57]. Briefly, the FID detector was set at $300^{\circ} \mathrm{C}$, and the sample was injected at $200^{\circ} \mathrm{C}$ with a split ratio of $5: 1$. The oven temperature program was $3 \mathrm{~min}$ at $50^{\circ} \mathrm{C}$, followed by an increase of $10^{\circ} \mathrm{C} / \mathrm{min}$ to $250^{\circ} \mathrm{C}$ for $2 \mathrm{~min}$. Ion analyses were done by liquid chromatography as previously described [200].

DNA extraction from microcosm samples was done as previously described [200]. Briefly, samples were filtered and filters were crushed with sterile toothpicks. Total DNA was extracted using the MoBio Powerlyzer DNA isolation kit (MoBio, CA, USA). DNA was stored at $-80^{\circ} \mathrm{C}$ until further molecular analyses. Quantification of $16 \mathrm{~S}$ rRNA genes of total bacteria as well as members of the family Peptococcaceae, and of the catabolic genes $\operatorname{bam} A, b s s A$ and $a b c A$ was done as previously described [57]. The intergenic region between the putative benzene carboxylase gene, $a b c A$, and the putative benzoate coenzyme A ligase $(b z l A)$ was amplified using primer pair abca1296f (5'GGTTTGCTTTGACTGCACCT-3') and bzla268r (5'-GAATCCTGTCCTCCGTTTCA-3') as previously described [114]. Amplification was performed in a $25 \mu$ l reaction mixture containing $0.6 \mu \mathrm{M}$ of each primer, $3 \mu \mathrm{l}$ template DNA, $6.5 \mu \mathrm{l}$ MilliQ and $12.5 \mu \mathrm{l} 2 \mathrm{x}$ IQ SYBR Green Supermix (Bio-Rad, CA, USA) and using a CFX96 real-time PCR machine (Bio-Rad). For this assay no calibration line was made, and thus, exclusively information regarding the presence or absence of this intergenic region could be obtained.

\section{First-order degradation rate constant calculation}

First-order degradation rate constants $(\mathrm{k})$ were calculated according to Equation 5.1 [111].

$k=\left(\frac{\log \left(x_{1}\right)-\log \left(x_{2}\right)}{t_{2}-t_{1}}\right)$

where $t_{2}-t_{1}$ is the time interval in days, $x_{1}$ is the concentration at time $t_{1}$ and $x_{2}$ is the concentration at time $t_{2}$. 


\section{Chapter 5.}

\section{$\underline{\text { Results }}$}

\section{Microcosm experiments using liquid from the continuous culture}

The microcosm containing both medium and liquid from the continuous culture $(1: 1 \mathrm{v} / \mathrm{v})$ degraded benzene at a first order degradation rate constant of 0.01 day $^{-1}$ corresponding with a rate of $0.64 \mu \mathrm{M} /$ day.

In the microcosm containing liquid from the continuous culture, five doses of 100 $\mu \mathrm{M}$ of benzene were degraded over 550 days with a first order rate constant of $0.04 \pm 0.02$ day $^{-1}(1.6 \pm 0.4 \mu \mathrm{M} /$ day) (Figure 5.1). The ratio of consumed benzene to nitrate was $1: 10$.

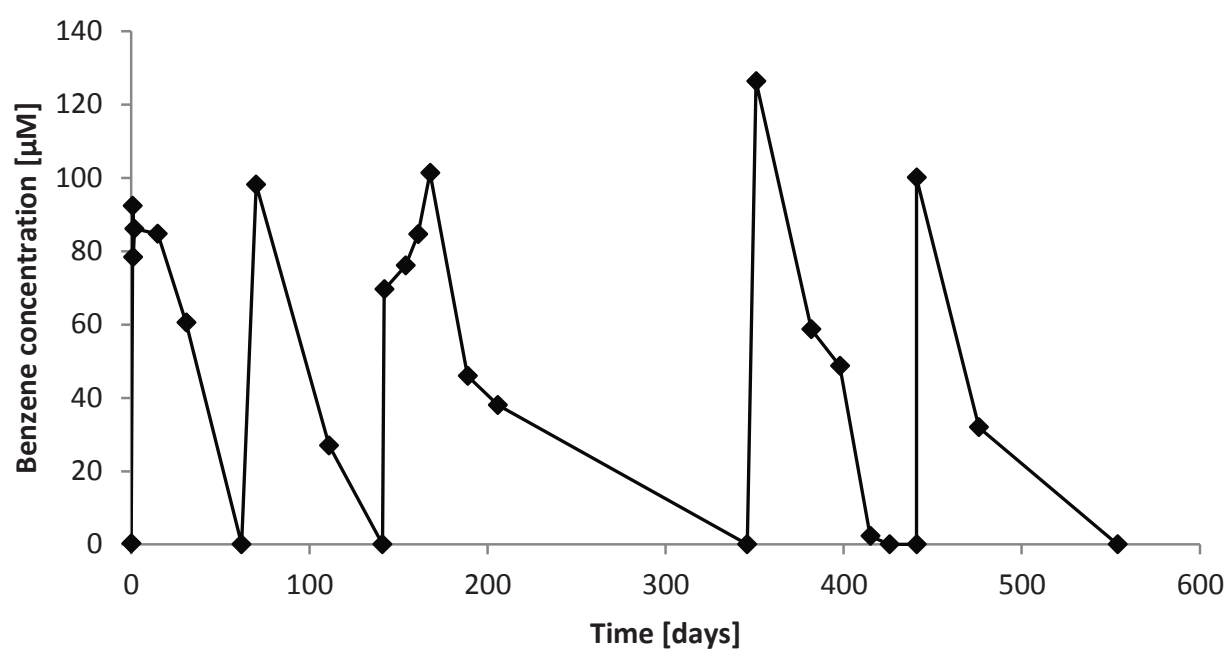

Figure 5.1 Benzene degradation in a microcosm with liquid from the continuous culture.

\section{Bioaugmentation experiments using groundwater from a tar contaminated site}

In the following, different ways of inoculation of the microbial continuous culture community to the groundwater from a tar-contaminated site were tested. Benzene was degraded in microcosms with autoclaved groundwater plus living inoculum consisting of a benzene degrading microbial community filtered from continuous culture liquid, retentostat liquid, medium plus filtered cells, liquid of the continuous culture, or continuous culture biofilm (Table 5.2). Benzene was not degraded in sterilized groundwater microcosms. Benzene was degraded with an initial first order rate constant $(\mathrm{K})$ of $2.0 \pm 0.3 \times 10^{-3} \mathrm{day}^{-1}$ in microcosms containing groundwater from a tar contaminated site with medium, without inoculated microorganisms. No further benzene degradation was observed in these microcosms after 118 
321 days. In contrast, complete benzene degradation $(100 \mu \mathrm{M})$ was observed in the microcosms inoculated with liquid or biofilm cells from the continuous culture with the exception of the microcosms inoculated with filtered cells.

Table 5.2 Benzene first order degradation rate constant $\left[\right.$ day $\left.^{-1}\right]$ in the bioaugmentation microcosms using groundwater from a tar contaminated site.

\begin{tabular}{lc}
\hline \multicolumn{1}{c}{ Condition } & $\mathbf{K}^{\prime}\left[\mathbf{d a y}^{-1}\right]$ \\
\hline Sterile groundwater & - \\
Sterile groundwater+inoculum & $6.6 \pm 4.9 \times 10^{-3}$ \\
Autoclaved groundwater+living inoculum & $10.0 \pm 5.1 \times 10^{-3}$ \\
Retentostat liquid & $6.0 \pm 3.5 \times 10^{-3}$ \\
Continuous culture liquid & $2.0 \pm 0.3 \times 10^{-3}$ \\
Medium & $18.6 \pm 3.1 \times 10^{-3}$ \\
Medium+filtered cells & $2.7 \pm 0.7 \times 10^{-3}$ \\
Living inoculum using filtered cells & $11.2 \pm 8.8 \times 10^{-3}$ \\
\hline
\end{tabular}

- $\quad$ Means no benzene degradation observed within 320 days

The microcosms with only groundwater from the location plus medium did not contain Peptococcaceae 16S rRNA nor abcA genes 230 days after inoculation ( $<119$ gene copies/ $\mu 1$ sample) (Figure 5.2). The microcosms containing filtered cells of the continuous culture without medium did not contain bamA genes ( $<6$ gene copies/ $\mu 1$ sample). The microcosms with addition of medium and biofilm inoculum from the continuous culture contained the highest 16S rRNA gene copy numbers for Peptococcaceae $\left(15 \pm 4.0 \times 10^{6}\right.$ gene copies/ml sample), abcA (14 $\pm 7.2 \times 10^{7}$ gene copies $\left./ \mathrm{ml}\right)$, bamA $\left(44 \pm 6.3 \times 10^{6}\right.$ gene cop$\mathrm{ies} / \mathrm{ml})$, and $b s s A$ genes $\left(1.8 \pm 1.5 \times 10^{6}\right.$ gene copies $\left./ \mathrm{ml}\right)$. 
Chapter 5.

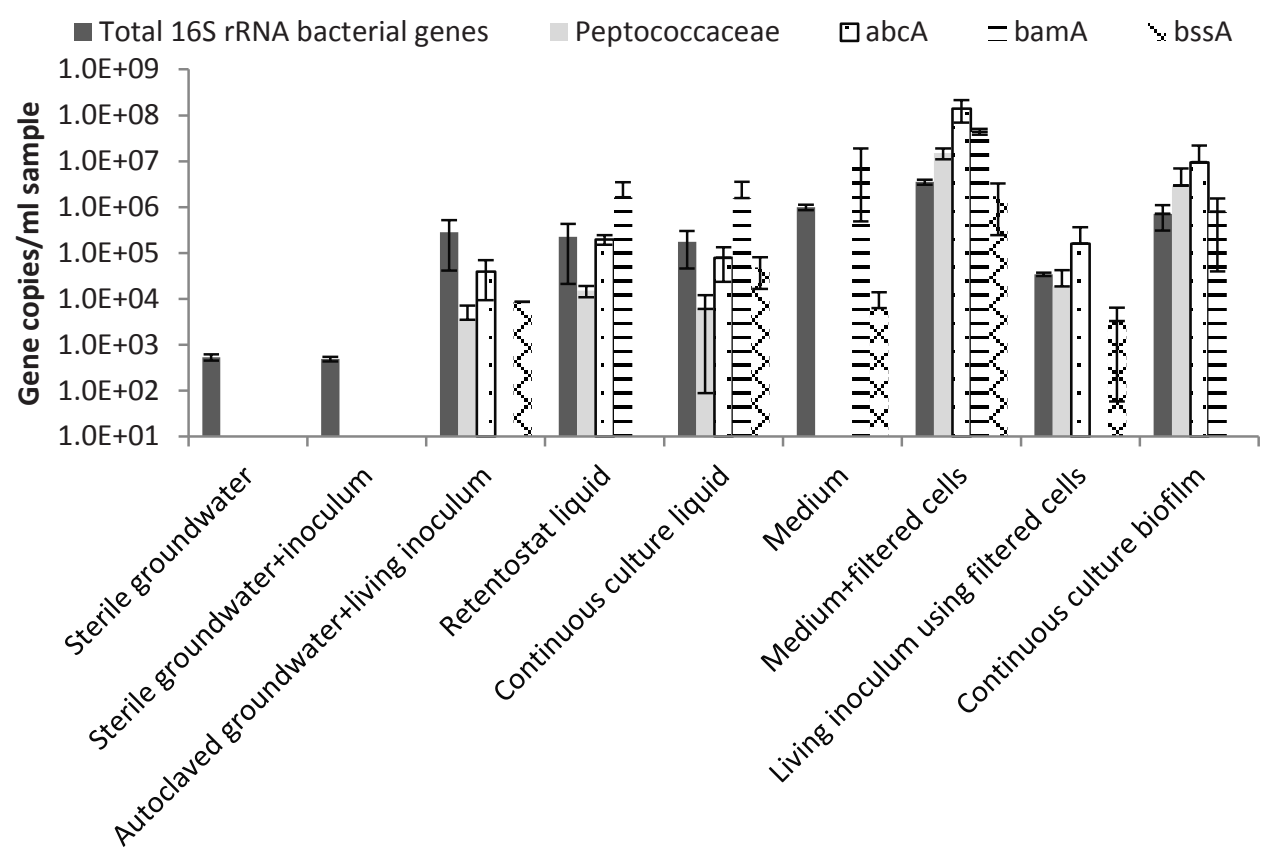

Figure 5.2 qPCR analyses of 16S rRNA genes of total bacteria and Peptococcaceae and functional genes $a b c A$, bamA and $b s s A$ in the bioaugmentation microcosms using groundwater from a tar contaminated site after 230 days of inoculation. Error bars represent standard deviations based on analytical replicates.

\section{Benzene-degrading isolates from the continuous culture}

Isolation of bacterial species from the continuous culture was done to characterize the main players and associated mechanisms and pathways in the anaerobic benzene degradation. Furthermore, these isolates might be used in bioaugmentation studies. Five bacterial species were isolated from the continuous culture. In this study, we isolated strains most closely related to Bacillus cereus, Ochrobactrum anthropi, Comamonas badia and Mesorhizobium plurifarium with $100 \%, 99 \%, 97 \%$ and $98 \%$ identity based on their $16 \mathrm{~S}$ rRNA gene sequence, respectively. Previously, Pseudomonas stutzeri strain BN was isolated on benzene with nitrate [47]. Bacillus sp. strain BOC1 was isolated on crotonate or glucose. Comamonas sp. strain BOC3 and Ochrobactrum sp. strain BOC2 were isolated on crotonate. Bacillus sp. strain BOC1, Comamonas sp. strain BOC3 and Ochrobactrum sp. strain BOC2 were able to grow on benzene with nitrate. From the same continuous culture one nitrate- 
Isolation of anaerobic benzene degrading bacteria reducing pure culture of Mesorhizobium sp. strain B3 was obtained that was unable to degrade benzene, but capable of growth on potential benzene degradation metabolites, i.e. phenol, benzoate and 4-hydroxybenzoate. The growth of the pure cultures on different electron donors and acceptors is described in Table 5.3. Growth on glucose was possible without an electron acceptor for Comamonas sp. strain BOC3, Mesorhizobium sp. strain B3, Bacillus sp. strain BOC1 and Ochrobactrum sp. strain BOC2. 
Chapter 5.

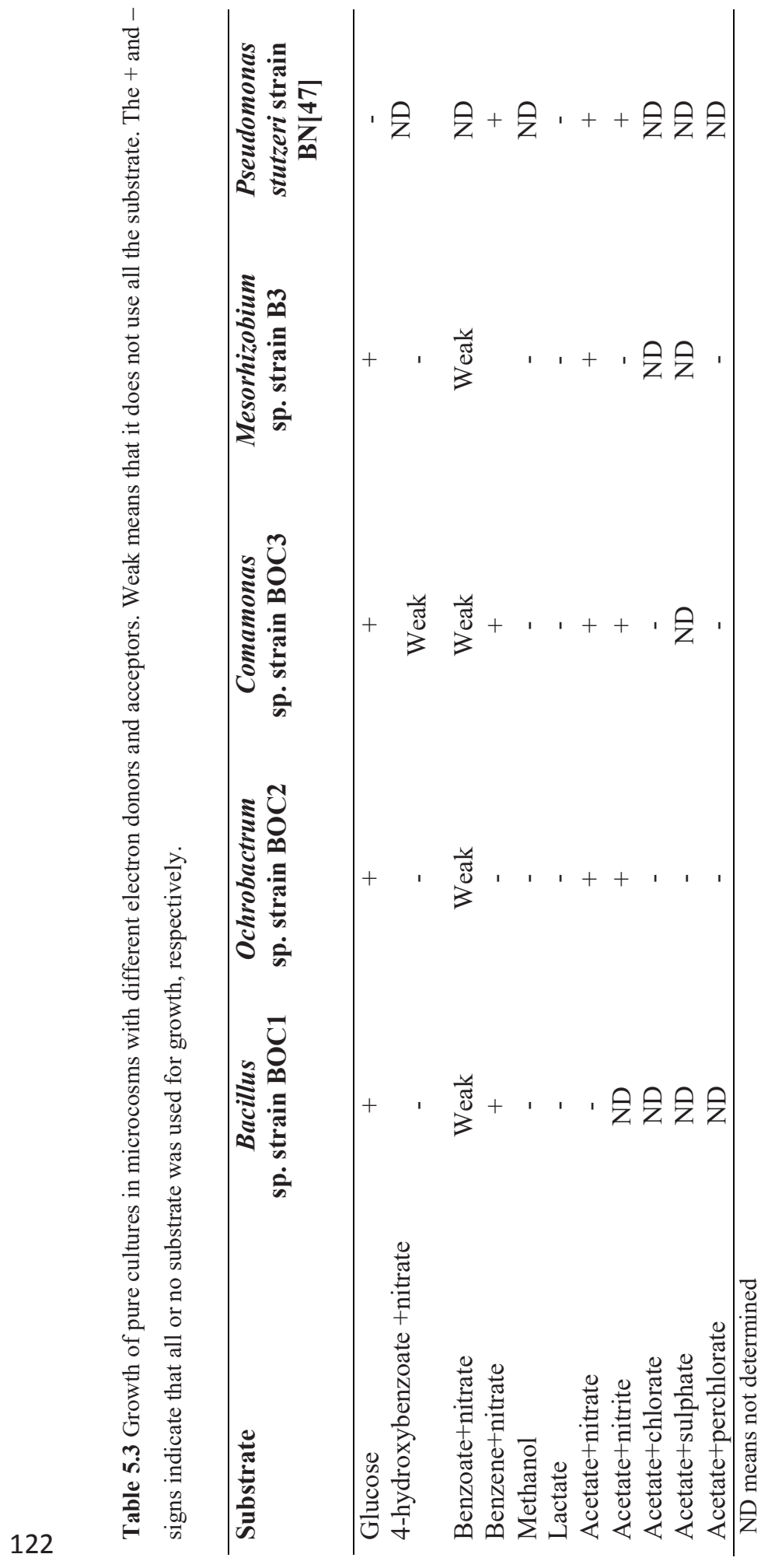


After maintaining Comamonas sp. BOC3 for four years on propionate, the isolate degraded benzene at a rate of $4.8 \times 10^{-2}$ day $^{-1}$. In contrast, after maintaining Bacillus sp. strain BOC1, Mesorhizobium sp. strain B3, Ochrobactrum sp. strain BOC2 and Pseudomonas sp. strain $\mathrm{BN}$ for four years in batch cultures on propionate, pyruvate or acetate, neither of these strains degraded a spike of $50 \mu \mathrm{M}$ benzene within 132 days in the presence of nitrate. Using qPCR the benzene carboxylase-encoding $a b c A$ gene was detected in the initial pure cultures of Bacillus sp. strain BOC1, Ochrobactrum sp. strain BOC2, Mesorhizobium sp. strain B3 and Comamonas sp. BOC3 maintained on benzene for four years at concentrations of $14.7 \pm 2.0 \times 10^{2}, 7.0 \pm 6.0 \times 10^{2}, 14.5 \pm 5.2 \times 10^{2}$ and $20.8 \pm 1.9 \times 10^{3}$ gene copies $/ \mathrm{ml}$ culture, respectively. In contrast, this gene could no longer be detected in the DNA of the pure cultures maintained on propionate, pyruvate or acetate, with the exception of Comamonas sp. BOC3, where low concentrations of $a b c A$ were still detectable (Table 5.4). The intergenic region between the $a b c A$ gene and the $b z l A$ gene encoding a putative benzoate coenzyme A ligase was neither detected in cultures of Comamonas sp. BOC3, Bacillus sp. strain BOC1, Ochrobactrum sp. strain BOC2 and Mesorhizobium sp. strain B3 maintained on benzene nor in cultures maintained for four years without benzene. In contrast, this intergenic region was detectable using DNA extracted from continuous culture biofilm (data not shown). 
Chapter 5.

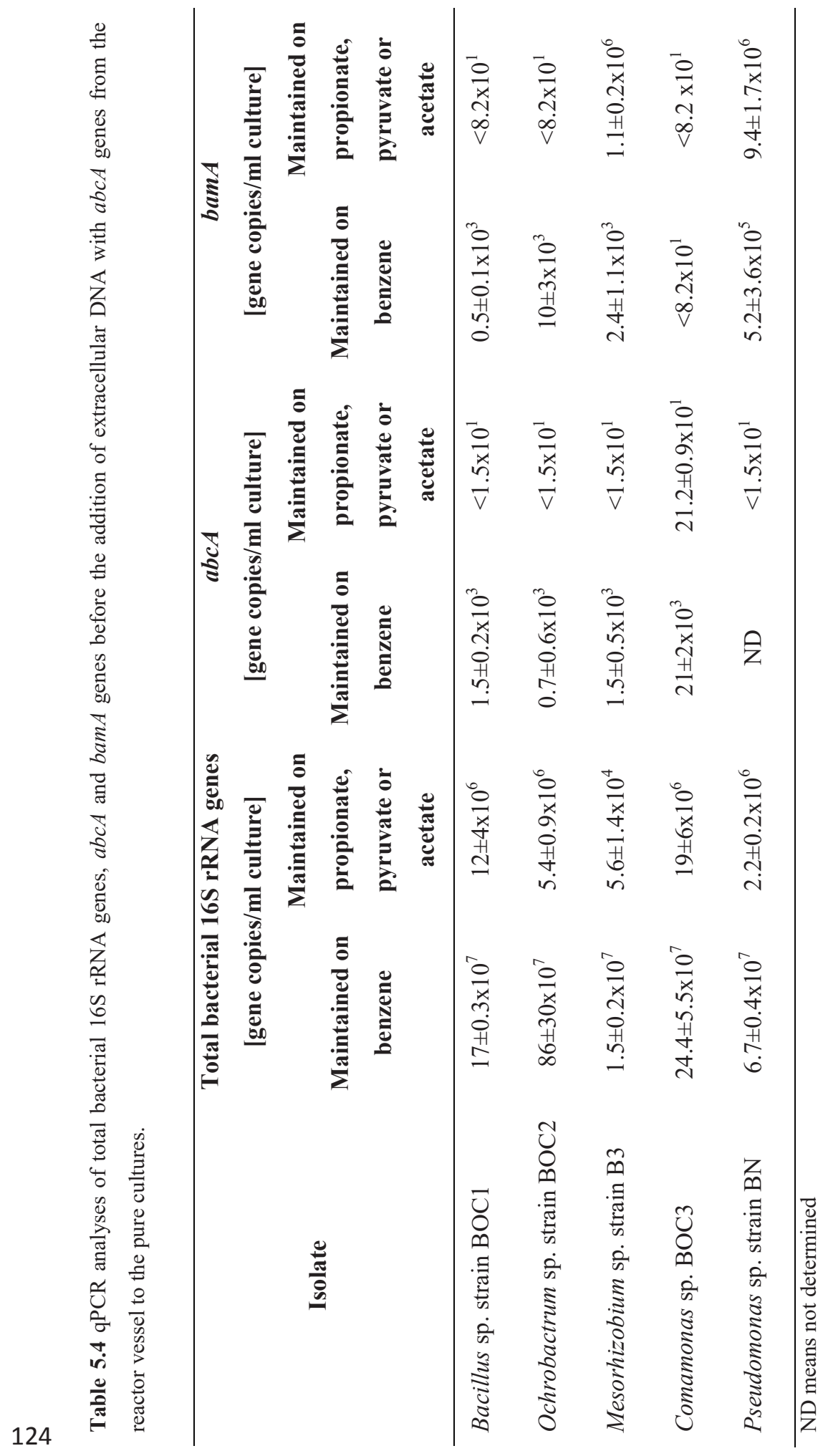




\section{Transfer of extracellular $a b c A$ gene}

After the addition of extracellular DNA containing the $a b c A$ gene from the continuous culture to the pure cultures, no benzene degradation was observed within 194 days for $\mathrm{Ba}$ cillus sp. strain BOC1, Mesorhizobium sp. strain B3 and Ochrobactrum sp. strain BOC2, respectively. In contrast, at 34 days after extracellular DNA addition to the microcosm with Pseudomonas sp. strain BN, this strain degraded benzene with a first order degradation rate constant of $3.0 \times 10^{-2}$ day $^{-1}$. A reference microcosm of a Pseudomonas sp. strain BN without the addition of extracellular DNA was not able to degrade benzene within 239 days.

\section{Discussion}

The goal of this study was to determine the transferability of anaerobic benzene degradation capacity of a microbial community which has been enriched in a continuous biofilm reactor with nitrate as electron acceptor for more than 15 years [57].

\section{Microcosm experiments using liquid from the continuous culture}

Interestingly, the highest benzene degradation rate constant of $0.04 \pm 0.02$ day $^{-1}$ was determined using exclusively liquid from the continuous culture. In a microcosm with both medium and liquid from the continuous culture, benzene was degraded at a lower rate constant of 0.01 day $^{-1}$. We hypothesize that growth on vitamins supplied in the medium of the microcosms may have lowered the benzene degradation rate. This suggestion is in line with previous results based on protein concentrations suggesting lower benzene degradation rates due to fewer benzene degraders and potential growth of organisms on vitamins in a continuous biofilm culture [57].

The fact that the putative intergenic region between $a b c A$ and benzoate coenzyme A ligase encoding $b z l A$ was detected besides the $a b c A$ gene in the continuous culture biofilm is in line with previous studies indicating benzene degradation through an initial carboxylation $[57,114]$.

\section{Bioaugmentation experiments using groundwater from a tar contaminated site}

The benzene degradation rates differed highly between microcosms with different inocula. First order degradation rate constants ranged from $2.0 \pm 0.3 \times 10^{-3}$ to $18.6 \pm 3.1 \times 10^{-3}$ day $^{-1}$. Interestingly, the microbial community in microcosms from the continuous culture degrad- 


\section{Chapter 5.}

ed benzene up to a first order degradation rate constant of $0.04 \pm 0.02$ day $^{-1}$. This difference in benzene degradation rates might by caused by the influence of groundwater from a tar contaminated site. The microbial community in the microcosms containing biofilm aggregates and medium with filtered cells as inoculum degraded the benzene with the shortest half-life. These results suggest that it is important to have continuous culture biofilm to obtain successful bioaugmentation. Previous studies also suggested that the presence of biofilm, containing high amounts of bacterial cells, increased benzene degradation rates [43, 57]. The results of our study indicate that the biodegradation rate can also be increased by the supply of nutrients from the medium to groundwater. This result is important for the use of bioaugmentation with this biofilm culture at the tar-contaminated field site. It is recommended to inject the inoculum with medium containing the necessary nutrients into the contaminated subsurface.

Both microcosms with groundwater plus medium, without inoculum did neither contain Peptococcaceae nor $a b c A$ genes at levels above the detection threshold of $1.5 \times 10^{1}$ gene copies/ml culture. This indicates that the groundwater upstream of the contaminated tar plume did not contain these organisms and genes coding for enzymes thought to be important for anaerobic benzene degradation [43, 57, 115]. In contrast, the microcosms containing inoculation material from the benzene degrading denitrifying continuous culture contained both Peptococcaceae 16S rRNA and $a b c A$ genes. Hence, the addition of benzene degrading organisms (bioaugmentation) may be a useful method to enhance benzene degradation in situ. Interestingly, the microcosms containing filtered cells without medium did not contain bamA genes in contrast to microcosms containing the same inoculum but to which medium was added. This may suggest that the organisms that possess this bamA gene, involved in reductive benzene ring cleavage [118], depend on nutrients either provided directly with the medium or produced by other organisms only in the presence of the medium. Continuous culture biofilm material together with medium appeared to be the best inoculation material for groundwater at this tar-contaminated location, based on both the high degradation rates and relatively high counts of Peptococcaceae, abcA, bamA and bss $A$ genes compared with the other inocula. 


\section{Benzene-degrading isolates from the continuous culture}

In this study several denitrifying, benzene-degrading isolates were obtained from the continuous culture. Bacillus sp. strain BOC1, Comamonas sp. strain BOC3, Ochrobactrum sp. strain BOC2 and Pseudomonas sp. strain BN [47] were able to grow on benzene with nitrate. We hypothesize that Mesorhizobium sp. strain B3 performed another process in the reactor vessel than benzene degradation since: (i) Mesorhizobium sp. strain B3 was not able to grow on benzene, (ii) Mesorhizobium sp. strain B3 was able to grow on 4hydroxybenzoate with nitrate suggesting that this organism might be responsible for degradation of benzene-derived metabolites, (iii) the intergenic region of the $a b c A$ gene and the putative benzoate coenzyme A ligase encoding gene bzlA was not amplified from genomic DNA of Mesorhizobium sp. strain B3 and (iv) the detection of bamA genes involved in reductive benzene ring cleavage [118]. Interestingly, after maintaining the cultures for several years on pyruvate or acetate instead of benzene, Bacillus sp. strain BOC1, Ochrobactrum sp. strain BOC2, and Pseudomonas sp. strain BN lost their ability to degrade benzene. This result is in line with the fact that the benzene carboxylase gene, $a b c A$, was no longer detected in the DNA of these isolates after maintaining them on pyruvate or acetate, whereas this gene was detected in all cultures when maintained on benzene. In contrast, Comamomas sp. strain BOC3 was still able to degrade benzene, albeit at a low rate constant of $4.8 \times 10^{-2}$ day $^{-1}$, in line with detection of residual concentrations of the $a b c A$ gene (Table 5.4). Based on the result that the organisms lost the ability to degrade benzene and the fact that there were genes encoding phage-related proteins and transposable elements on the same contig as the $a b c A$ gene, it is tempting to speculate that the $a b c A$ gene might be or at least has been located on a mobile element and thus subject to horizontal gene transfer [215]. Unfortunately, the addition of extracellular DNA from the continuous culture containing $a b c A$ genes did not initiate benzene degradation in the pure cultures of Bacillus sp. strain BOC1, Ochrobactrum sp. strain BOC2 and Mesorhizobium sp. strain B3. In contrast, after the addition of the extracellular continuous culture DNA with $a b c A$ genes to a nonbenzene degrading Pseudomonas sp. strain BN culture, this isolate regained its ability to degrade benzene. This result is in line with previous studies showing that the species Pseudomonas stutzeri has type IV pili, which facilitate the uptake of extracellular DNA in a DNase I-resistant state during competence and the potential for natural genetic transformation $[220,221]$. The fact that the $a b c A$ gene was not taken up by the other pure cultures 


\section{Chapter 5.}

might be due to (i) the lack of pilT homologs encoding proteins needed to take up extracellular DNA [222], (ii) degradation of the $a b c A$ genes by extracellular nucleases before they could be taken up [223], or (iii) lack of functional expression in these organisms. Further experiments beyond the scope of the study described here will be needed to improve our understanding on the potential mobility of $a b c A$ containing genetic elements and their uptake in order to explore the potential for gene bioaugmentation. A better understanding of the potential for horizontal transfer of the $a b c A$ gene and the exact mechanism of mobilisation would enable more effective bioremediation through bioaugmentation. Horizontal gene transfer between prokaryotes may occur through transformation, transduction and conjugation [224]. Hence, bioaugmentation using anaerobic benzene degrading bacteria might increase the benzene degradation potential in situ because (i) the augmented bacteria grow in situ and/or (ii) their DNA that encodes the anaerobic benzene degrading capacity is transferred to members of the indigenous microbial community.

This study suggests that bioaugmentation using laboratory cultivated organisms might be useful to stimulate benzene degradation in contaminated groundwater systems. While being beyond the scope of this study, it would be interesting to determine the effect of gene bioaugmentation at contaminated sites. Further field studies and dedicated pilot testing could help to elucidate the potential use of horizontal gene transfer in bioremediation studies. 


\section{Supplementary Figures and Tables}

Table S5.1 Overview of the characteristics of the groundwater used to prepare microcosms.

Parameter

Unit

Value

\begin{tabular}{lcc}
\hline Temperature & ${ }^{\circ} \mathrm{C}$ & 13 \\
$\mathrm{pH}$ & & 5.6 \\
Conductivity & $\mu \mathrm{S} / \mathrm{cm}$ & \\
Redox & $\mathrm{mV}$ & 152 \\
Oxygen & $\mathrm{mg} / 1$ & 0.3 \\
Total organic carbon & $\mathrm{mg} / 1$ & 6.1 \\
Nitrite & $\mathrm{mg} / 1$ & 0 \\
Nitrate & $\mathrm{mg} / 1$ & 24.1 \\
Sulphate & $\mathrm{mg} / 1$ & 35.6 \\
Iron (total) & $\mathrm{mg} / 1$ & 0.04 \\
\hline
\end{tabular}


Chapter 5. 
Chapter 6. General discussion and perspectives 


\section{Chapter 6.}

\section{General discussion and perspectives}

In the environment, chemicals enter the subsurface due to anthropogenic activities. At many locations the subsurface is highly contaminated with a diverse range of organic compounds such as polycyclic aromatic hydrocarbons (PAH), benzene, toluene, ethylbenzene and xylene (BTEX). Furthermore, the increased use of biofuels in the biobased economy is becoming an area of concern. The emphasis of this thesis was placed on biodegradation of both fossil- and biobased fuel-derived contaminants. Selected contaminants included fossilbased fuel derived compounds, i.e. MtBE and benzene and a biobased fuel derived compound, i.e. EtBE. TBA is being produced as metabolite during MtBE and EtBE degradation. Both these fossil- and biobased compounds easily dissolve in groundwater, are toxic and pose a risk to human, animal and environmental health. Therefore, it is imperative to remove these contaminants from the subsurface. A sustainable and cost-effective clean-up method is bioremediation [92]. The three main bioremediation techniques, namely (i) natural attenuation, (ii) biostimulation and (iii) bioaugmentation were investigated in the studies described in this thesis. An important aspect of this thesis is the simultaneous presence of both fossil- and biobased contaminants in groundwater. Since many contaminated sites in the subsurface are anoxic, the focus of this thesis was on anaerobic biodegradation. To this end, Chapter 1 of this thesis gives an overview of the current state of the art with respect to anaerobic MtBE, EtBE, TBA and benzene bioremediation studies.

The aim of the studies described in this thesis was to gain more insight in biodegradation of MtBE, EtBE, TBA and benzene using biostimulation (different nutrients and electron acceptors) and bioaugmentation with laboratory-cultivated microbial communities.

\section{Biodegradation capacity of MtBE, EtBE TBA and benzene}

\section{Benzene}

In Chapter 2 the highest anaerobic benzene degradation rates were found when compared with any previous studies. Bacteria belonging to the family of the Peptococcaceae were confirmed to play an important role in the benzene degradation as based on 16S rRNA gene targeted community composition analysis. Also members of the Anaerolineaceae were suggested to be involved in benzene or benzene metabolite degradation. More recently, members of the family Anaerolineaceae were not among the most active populations as based on metatranscriptome analysis of the same benzene-degrading community [215]. 
This difference between relative abundance and activity of microbes has been described previously [225]. Hence, integrating DNA-based community profiling, metagenomics and metatranscriptomics, in combination with stable isotope probing strategies [43] will give the best overview of the composition, functional capacity as well as activity of the microbial populations involved in the degradation of specific contaminants. Chapter 2 also describes the development of specific qPCR assays targeting members of the family Peptococcaceae and the benzene carboxylase gene $(a b c A)$.

\section{$\underline{\mathrm{MtBE} \text { and TBA }}$}

MtBE was degraded in groundwater microcosms to TBA, other metabolites and/or $\mathrm{CO}_{2}$ under natural conditions, and with the addition of medium, ferrihydrite or sulphate (Chapter 3 ) in line with the fact that iron- and sulphate-reducing conditions prevail in the contaminated groundwater at an industrial refinery from which the inoculum was obtained. Since MtBE was degraded under conditions intrinsic to the field site it might not be necessary to stimulate the MtBE degradation process at the field site. Nevertheless, this hypothesis needs to be confirmed in the field because of different temperatures, different electron acceptor concentrations or other (unknown) factors that differ between the field and the laboratory. It was observed in this study that the addition of nitrate and chlorate hindered MtBE degradation in the microcosms. Furthermore, it was suggested to isolate organisms involved in the anaerobic MtBE degradation in order to provide the necessary basis for developing molecular diagnostic assays as monitoring tool for anaerobic MtBE degradation in the subsurface. In this study, dilution to extinction cultivation was performed to isolate MtBE degrading strains. Unfortunately, no pure culture was obtained, in line with the fact that to date, no anaerobic MtBE degrading isolates have been obtained [24]. It has been previously suggested that this may be due to the fact that anaerobic MtBE degradation may require the interaction of several organisms [24, 88].

The study described in Chapter $\mathbf{3}$ also indicated that TBA was cometabolically depleted with MtBE and MtBE substrate analogues, i.e. syringate, vanillate or ferulate [200]. The addition of such methoxylated compounds may be useful to stimulate TBA degradation in situ. MtBE was converted mainly to TBA. So TBA degradation should be stimulated. In order to test this hypothesis in the field, it will be important to determine the 


\section{Chapter 6.}

amount of methoxylated compounds needed to induce cometabolic TBA degradation, since these compounds can be rather expensive to use.

\section{EtBE}

Results presented in Chapter 3 indicated that cometabolic EtBE depletion with ferulate, syringate, isopropanol or diethyl ether, as primary growth substrates, occurred. To date, only a limited number of studies on anaerobic EtBE biodegradation have been described in literature. Stimulation of anaerobic EtBE degradation in microcosms with contaminated groundwater by the addition of nutrients or electron acceptors did not work (Chapter 4). This is in slight contrast to a previous study that showed stimulation of EtBE degradation by nitrate addition [21]. Since EtBE is assumed to be a rather 'new' biobased contaminant it is expected that microbial communities need adaptation time to degrade this chemical in the environment, depending on the site from which the inoculum is obtained. The nature of adaptation to environmental contaminants is relatively unknown for most compounds except for intentionally added toxic chemicals such as pesticides and antibiotics [226]. Evolved adaptation to such chemicals is common by for example membrane repair or morphological changes [226, 227]. Genes and the encoded enzymes that are important in the transformation of EtBE can be identified using metagenomics and metatranscriptomics by comparing for example different communities that are or are not exposed to EtBE. Such experiments would reveal more information on potential anaerobic and aerobic EtBE degrading microorganisms.

To determine whether anaerobic EtBE degradation would be thermodynamically feasible, the difference in electron potential is determined. The half reaction for the complete oxidation of EtBE in an aqueous environment to $\mathrm{CO}_{2}$ and $\mathrm{H}_{2} \mathrm{O}$ is given in Equation 6.1.

$\mathrm{C}_{6} \mathrm{H}_{14} \mathrm{O}+11 \mathrm{H}_{2} \mathrm{O} \rightarrow 6 \mathrm{CO}_{2}+36 \mathrm{e}^{-}+36 \mathrm{H}^{+}$

The Gibbs free energy of formation at $\mathrm{pH} 7$ for all compounds described in Equation 6.1 is $-126.8 \mathrm{~kJ} / \mathrm{mol},-237.1 \mathrm{~kJ} / \mathrm{mol},-394,4 \mathrm{~kJ} / \mathrm{mol}$ and $-40.0 \mathrm{~kJ} / \mathrm{mol}$ for EtBE, $\mathrm{H}_{2} \mathrm{O}, \mathrm{CO}_{2}$ and $\mathrm{H}^{+}$, respectively [228]. The total reaction energy is $1048.7 \mathrm{~kJ} / \mathrm{mol}$. The electron potential difference is calculated with Equation 6.2 using the Faraday constant (F; 96485 coulombs/mol electrons). 
$\mathrm{dG}=-\mathrm{n} \times \mathrm{F} \times \mathrm{dE}$

where $\mathrm{dG}$ is the Gibbs free energy $[\mathrm{kJ} / \mathrm{mol}], \mathrm{n}$ is the amount of electrons in the half reaction, $\mathrm{F}$ is the Faraday constant [96485 coulombs/mol electrons] and $\mathrm{dE}$ is the electron potential difference of the reaction [V]. Using Equation 6.2, the electron potential difference of the conversion of EtBE to $\mathrm{CO}_{2}$ and $\mathrm{H}_{2} \mathrm{O}$ is $0.30 \mathrm{~V}$. Table 6.1 provides the standard reduction potential for the different potential electron acceptors given.

Table 6.1. Standard reduction potentials at $25^{\circ} \mathrm{C}$ for the used electron acceptors in this study [229].

\section{Chemical half reaction}

\begin{tabular}{lc}
\hline $\mathrm{ClO}_{3}^{-}(\mathrm{aq})+6 \mathrm{H}^{+}+6 \mathrm{e}^{-} \rightarrow \mathrm{Cl}^{-}(\mathrm{aq})+3 \mathrm{H}_{2} \mathrm{O}$ & 1.03 \\
$2 \mathrm{NO}_{3}^{-}(\mathrm{aq})+1.2 \mathrm{H}^{+}+10 \mathrm{e}^{-} \rightarrow \mathrm{N}_{2}(\mathrm{~g})+0.6 \mathrm{H}_{2} \mathrm{O}$ & 0.74 \\
$\mathrm{Fe}^{3+}(\mathrm{aq})+\mathrm{e}^{-} \rightarrow \mathrm{Fe}^{2+}(\mathrm{aq})$ & 0.2 \\
$\mathrm{SO}_{4}^{2-}(\mathrm{aq})+9 \mathrm{H}^{+}+8 \mathrm{e}^{-} \rightarrow \mathrm{HS}^{-}(\mathrm{aq})+4 \mathrm{H}_{2} \mathrm{O}$ & -0.22 \\
\hline
\end{tabular}

Under nitrate and chlorate reducing conditions, EtBE degradation would be thermodynamically feasible. The difference in electron potential under sulphate- and iron(III) reducing conditions is much smaller, and thus the EtBE degradation with sulphate and iron(III) will yield less energy compared with the other acceptors. It should be noted, however, that aspects such as activation energy and conversion kinetics were not taken into account. Since the anaerobic degradation of EtBE seems at least thermodynamically feasible with the different electron acceptors, it is conceivable that anaerobic EtBE degrading enrichments can be obtained by setting up microcosms using groundwater from a range of different locations.

The micro-oxic degradation of EtBE using algae might be of great interest for waste water treatment plants (Chapter 4). Self-oxygenation in combined algal-bacterial systems could avoid (i) the use of costly air sparging which is limited by the low solubility of oxygen in water, (ii) volatilization of hazardous organic molecules and (iii) toxic sprays of organisms and compounds [230]. The degradation rate and total mass of EtBE degraded is significantly affected by light intensity and mixing [231]. Optimisation of these parame- 


\section{Chapter 6.}

ters will increase the efficiency of the system. Interaction between algae and bacteria also occurs in the dark with different results than under phototrophic conditions [232]. Wellmixed photobioreactors are recommended to protect the algae from pollutant toxicity and optimize light utilization efficiency. Algal-bacterial systems are characterised by a range of different interactions that might affect algal and/or bacterial growth [233], and include motility, chemotaxis, type IV secretion and quorum sensing [234, 235]. In addition, micronutrients such as vitamins and macronutrients such as nitrogen, oxygen and carbon are exchanged between algae and bacteria [232].

\section{$\underline{\text { A mixture of biobased and fossil-fuel derived contaminants }}$}

Contaminated locations often contain mixtures of different compounds. Several studies have investigated the antagonistic and synergistic effects of mixtures of fossil-based and biobased fuel components such as MtBE, TBA or benzene on the degradation of the single components [87, 165-168]. Different contaminants often co-occur in the subsurface resulting in preferential degradation of easily degradable substrates. Biobased and fossil-based contaminants may increasingly co-occur in the subsurface. Therefore, it is of great interest to study their combined degradation under different conditions. To determine the fate and transport of a mixture of biobased and fossil-based components in the subsurface, knowledge on the sorption behaviour of the different compounds is required [236]. To our knowledge, studies on the anaerobic degradation of a mixture of MtBE, EtBE, TBA and benzene have not been reported. Therefore in Chapter 3 a mixture of benzene, MtBE, EtBE and TBA was used to determine these antagonistic and synergistic effects.

In this chapter it was found that the addition of nitrate plus a benzene degrading community and chlorate (with and without medium containing nutrients and salts) stimulated benzene degradation, but hindered MtBE, EtBE and TBA degradation. This suggests that different contaminants may need different (redox) conditions for degradation. Therefore, knowledge of the contaminants present in the subsurface and degradation under intrinsic conditions and possibilities for biostimulation under different redox conditions at the contaminated location is important in defining bioremediation strategies.

\section{Bioremediation of polluted environments}

In this thesis the degradation of MtBE, EtBE, TBA and benzene was investigated in laboratory experiments (Chapter 2-5). The translation of laboratory biodegradation studies to 
field-scale bioremediation studies is not straightforward. In the environment, many factors can affect the biodegradation potential such as bioavailability of the contaminants, seasonal changes in the conditions in the subsurface and the composition of indigenous microbial communities [110, 237, 238]. Besides this, many laboratory studies are performed at room temperature. Groundwater in the Netherlands between 5 and 50 meter is normally around $10-12^{\circ} \mathrm{C}$, causing a difference in biodegradation rates between the laboratory experiments and the field. Proper biological and chemical controls are therefore important to determine the effect that these environmental conditions have on the biodegradation.

Hydrocarbon molecules dissolved in the aqueous phase are readily available to microorganisms for degradation. In a fresh petroleum spill in the groundwater, however, only $2 \%$ of the introduced organic load may be dissolved in water [239], making degradation less favourable. A possible method to increase the solubility of contaminants is the addition of surfactants [240, 241] or the production of biosurfactants by microorganisms [242]. The contaminants in the aqueous phase will become mobile and conducive to microbial degradation.

Metabolite concentrations are important in determining degradation. However, these concentrations are often difficult to measure in the subsurface due to groundwater flow and the replenishment of contaminants from the pollution source. At complex spill sites it is often difficult to prove that a contaminant is degraded in groundwater [243]. The standard approach is to monitor contaminant concentrations at selected wells and calculate the mass loss over time. The shortcomings of this standard approach include: (i) the requirement of a dense network of monitoring wells, (ii) the need for monitoring for long times and (iii) the demand for a homogeneous aquifer [244]. Rapid developments in compound specific isotope analysis (CSIA) in field scale studies enables us to shed more light on the biodegradation of contaminants in the environment by revealing carbon and hydrogen fractionations. Stable isotope fractionation is used for the monitoring of in situ (bio)degradation of organic contaminants such as MtBE [245]. One- and two-dimensional CSIA provides information on in situ contaminant degradation and the reaction mechanisms [246]. Numerous studies have demonstrated the applicability of CSIA in in situ degradation of aromatics, chlorinated hydrocarbons and fuel oxygenates [246]. For example, CSIA was used at an EtBE contaminated field site revealing insignificant carbon isotope fractionation, but a low hydrogen isotope fraction of up to $+14 \%$ along an anoxic EtBE 


\section{Chapter 6.}

plume [22]. Based on these findings, it was suggested that EtBE was anaerobically degraded.

\section{Bioaugmentation test}

A biostimulation and bioaugmentation field study with nitrate $(35 \mathrm{mM})$ using a benzene degrading community (Chapter 2) is currently being performed at an oil-tar contaminated site. Both the microbial community from the continuous biofilm reactor and nitrate are added to the groundwater. In this field study the qPCR assays described in Chapter $\mathbf{2}$ are used to determine benzene degradation.

In order to prepare for this field study, continuous culture and microcosm experiments were performed as described in Chapter $\mathbf{5}$ of this thesis to determine the best bioaugmentation material. Results of this laboratory study showed the importance of biofilm material. Previous studies also suggested that both Peptococcaceae and abcA genes are important in the benzene degradation and that these benzene degraders and functional genes reside in the biofilm $[43,57]$. In Chapter $\mathbf{5}$ also the isolation of pure cultures of bacteria degrading benzene with nitrate is described.

\section{Continuous culture experiments}

To determine the effect of bioaugmentation of the continuous benzene degrading community at a field site contaminated with tar as described in Chapter 5, further experiments were performed as described in the following. Two experiments were performed with the continuous benzene degrading community (Chapter 2): (i) the injection of a $0.2 \mathrm{~g} / 1$ or $0.4 \mathrm{~g} / 1$ mixture of hydrocarbons (naphthalene, benzene, toluene, ethylbenzene, m-xylene, p-xylene, o-xylene, 1,2,4-trimethylbenzene, 1,2,3-trimethylbenzene and indene) and (ii) the addition of contaminated groundwater containing around $102 \mu \mathrm{M}$ benzene, $54 \mu \mathrm{M}$ toluene, $9 \mu \mathrm{M}$ ethylbenzene, $28 \mu \mathrm{M}$ meta- and para-xylene, $10 \mu \mathrm{M}$ styrene, $9 \mu \mathrm{M}$ o-xylene, $10 \mu \mathrm{M} 1,2,4$ trimethylbenzene, $4 \mu \mathrm{M}$ 1,2,3-trimethylbenzene, $34 \mu \mathrm{M}$ indene, $62 \mu \mathrm{M}$ naphthalene, $7 \mu \mathrm{M}$ 2-methylnaphthalene and $7 \mu \mathrm{M}$ 1-methylnaphthalene to the continuous culture.

The injection of the hydrocarbon mixture did not lead to increased concentrations of benzene, toluene, ethylbenzene, $o$-xylene, $p$-xylene, $m$-xylene or styrene over time measured with a GC-FID. This suggests that the microbial community that was maintained on benzene was able to degrade these hydrocarbons in the continuous culture. This result is 
in line with a previous study showing toluene degradation with a denitrifying, benzene degrading community [60]. However, in that study benzene degradation was initiated only after toluene was depleted. In the experiments described here, benzene and toluene were degraded simultaneously, but due to substrate limitation no preferred substrate can be determined.

The contaminated groundwater was added to the continuous culture at stepwise increased rates from 0.1 1/day to 1 1/day. This increased rate caused an increase in benzene headspace concentrations up to $30 \mu \mathrm{M}$ (Figure 6.1).

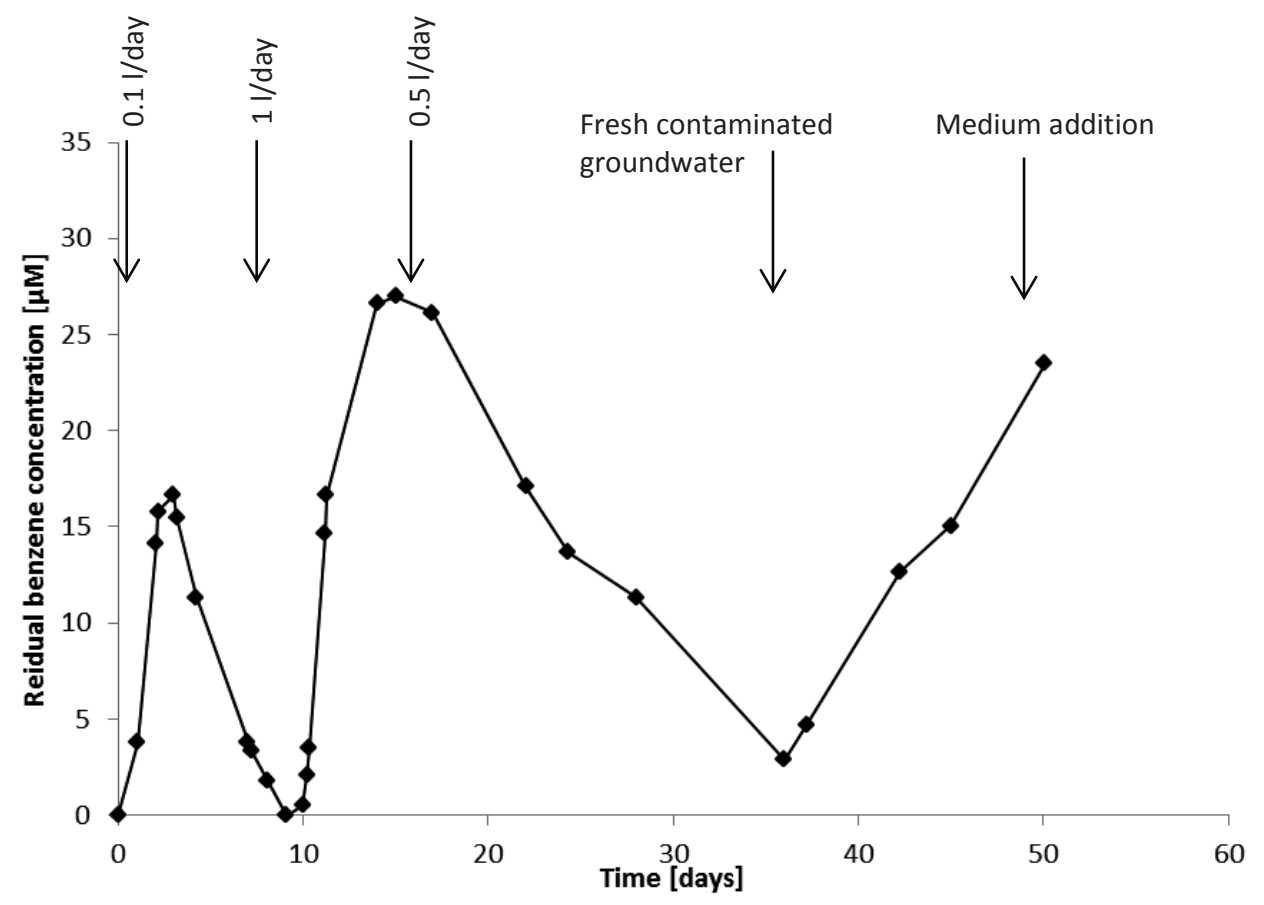

Figure 6.1 Residual benzene headspace concentration in the continuous culture over time with different pump rates as indicated with vertical arrows.

After 36 days fresh groundwater from the field site was connected to the continuous culture. Consequently, the residual benzene concentration increased indicating a cease of benzene degradation. The fact that no benzene degradation occurred when using this groundwater could be due to (i) high concentration of aromatic hydrocarbons or other toxic compounds, (ii) lack of nutrients and/or vitamins or (iii) inhibition of benzene degraders by indigenous microorganisms. The addition of the groundwater with a mixture of contaminants may have caused growth on more easily degradable substrates other than benzene in the groundwater. 


\section{Chapter 6.}

After 50 days the groundwater was replaced with medium as described in Chapter 2. Unfortunately, benzene degradation still ceased after 15 days, but addition of effluent, collected when the microbial community was degrading benzene, initiated benzene degradation. These results suggest that the microbial community in the contaminated groundwater was lacking nutrients, vitamins and/or metabolites formed by the microbial community which are necessary for benzene degradation.

\section{$\underline{\text { Microcosm experiment }}$}

Groundwater with nitrate or groundwater with nitrate possibly containing a benzene degrading community was extracted from infiltration water at the tar-contaminated field site. This groundwater was used to prepare microcosms. This microcosm experiment was performed to evaluate whether benzene degradation may occur when the groundwater with the indigenous community was augmented with the microbial community described in Chapter 2. Based on these results, no significant degradation of the target hydrocarbons occurred within 150 days.

\section{Connecting models with field- and laboratory data}

Due to the complexity of the subsurface, reactive transport modelling is often needed to reflect existing site conditions and to determine the effect of biodegradation [247]. Physicobio-geochemical processes such as advection, dispersion, sorption, ion exchange, volatilization, chemical reactions and biodegradation are involved in the transport of contaminants through the subsurface [248]. Models may give valuable information about the transport of contaminants, plume development and plume front locations [247, 249]. The incorporation of field and laboratory data such as rate constants and reaction equations in models is important. For example the analysis of field data led to a revised hydrogeological model as described in Chapter 3. The combined iterative use of models and experiments may give an assessment of the impacted area and the effect of bioremediation strategies. These models may also be used to scale up from local to regional scale, giving information on for example transport of contaminants to surface water (e.g. rivers and seas).

\section{Extracellular $a b c A$ gene and gene bioaugmentation}

As discussed in Chapter 5, a better understanding of the possibly mobile $a b c A$ gene in anaerobic benzene degradation would enable more effective bioremediation through gene 
bioaugmentation. Genes that encode enzymes needed for the degradation of organic compounds are often located on mobile genetic elements such as plasmids and transposons [238]. These genes can be spread by horizontal gene transfer into indigenous bacterial populations. For the use of gene bioaugmentation appropriate donor microorganisms should be selected [238]. Knowledge on the native bacterial community is also required such as the determination which genes are necessary to degrade the contaminant(s) and whether the indigenous population could take these genes up from the donor bacteria. Besides wholegenome sequencing to reveal the genes required for extracellular DNA uptake, proteomics can increasingly be used to aid in the search of transformable species [250]. The potential of using specific degradation genes in bioaugmentation studies offers new possibilities in bioremediation strategies. The $a b c A$ operon might be incorporated in the genome of a donor cell such as Escherichia coli or might be expressed in E. coli. E.coli might horizontally transfer the $a b c A$ gene to the indigenous population, supporting benzene degradation. To minimize the survival of genetically modified organisms (GMO) in the subsurface it is possible to implement a kill switch in the organism to self-destruct after it fulfilled its purpose [251]. Gene bioaugmentation with $E$. coli as donor has been successful in the degradation of 2,4 dichlorophenoxyacetic acid (2,4-D) [252]. To date, explicit gene bioaugmentation field studies are scarce in literature, since the difference between the effect of the donor cell or degradative mobile element is difficult to distinguish.

\section{Other emerging 'contaminants of the future'}

In this thesis the biodegradation of MtBE, EtBE, TBA and benzene was investigated. In the biobased economy, the environmental fate of biofuels is becoming an area of concern. Besides EtBE and TBA, biodiesel is increasingly being used. Currently, different types of biodiesel are used, including so-called first generation (produced from edible resources), second generation (produced from non-edible resources), third generation (algae production) and fourth generation (novel synthetic tools) biofuels [253, 254]. Initial microcosm experiments, using production water from a second generation biodiesel production plant, performed in the time frame of this study showed second generation biodiesel degradation under nitrate- and sulphate-reducing, as well as methanogenic conditions (data not shown). Biodiesel was degraded to lower chain fatty acid methyl esters (FAMEs), free fatty acids (FFAs) and/or $\mathrm{CO}_{2}$. More research has to be done to identify microbes and enzymes involved in anaerobic biodiesel degradation. 
Chapter 6.

\section{Concluding remarks and perspectives for 'contaminants of the future' degradation}

The research described in this thesis provided more insight in the degradation of both fossil- and biobased contaminants using biostimulation or bioaugmentation. Natural attenuation, biostimulation and bioaugmentation are considered cost effective, and these approaches can be expected to be increasingly used to remediate contaminated sites. Therefore, it is important to study the significance and possibilities of natural attenuation, biostimulation and bioaugmentation to maximize the bioremediation success. In this thesis it was hypothesized that MtBE, EtBE and TBA were cometabolically degraded with different substrate analogues. More research is needed to demonstrate if cometabolic degradation of MtBE, EtBE and TBA is indeed an important process during natural attenuation or if it can be applied for biostimulation at polluted environments. It is recommended to perform microcosm- as well as continuous culture experiments using both sediment and groundwater from a broad range of different locations to increase the possibility of obtaining MtBE, EtBE and TBA (co)metabolising enrichment cultures. Using continuous culture experiments, cometabolic substrate limitation experiments might be applied to identify and quantify the potential effect of competitive inhibition on degradation rates in mixtures [255].

To obtain MtBE, EtBE and TBA degrading organisms, new cultivation techniques based on in situ high-throughput cultivation of environmental microorganisms, such as an isolation chip (iCHIP), might aid in increasing the cultivable fraction of environmental microbes [103].

Rapid developments and decreasing costs in next generation sequencing (NGS) will enable its implementation more routinely to identify compositional and functional characteristics and dynamics of microbial communities at the contaminated site. Furthermore, whole-genome sequencing of isolates, metagenome sequencing as well as single cell genomics will be helpful in identifying and understanding the relevant microorganisms for bioremediation [256]. Revealing how different microorganisms function in contaminated subsurface environments is an important aspect of whole-genome sequencing. Understanding the physiological characteristics of the organisms is important in bioremediation studies. Based on the genome, the relevant growth substrates, nutrients and/or electron acceptors for biostimulation and cometabolic studies could be predicted. These could then be added at contaminated locations to stimulate bioremediation. 
Rapid developments in on site portable sequencing devices such as minION seems also promising [257]. The minION protocol does not require internet connection, making it suitable for field applications. Unfortunately, there are still challenges to improve this technique such as the low base accuracy and high sequencing error-rate [258, 259]. These challenges might be overcome with the development of new bioinformatic pipelines to analyse the raw sequencing data. With the minION rapid real-time-on-site DNA sequencing might be performed, increasing the opportunity for understanding the biodiversity in the field.

Monitoring is essential in natural attenuation, biostimulation and bioaugmentation studies. An interesting example are mobile qPCR DNA analysis devices, which may be used to analyse microbes on site [260]. Besides quantifying DNA biomarkers for specific bacterial, archaeal or algal groups, also genes encoding specific enzymes might be quantified. The relatively easy and fast use of such portable devices allows the on-site detection of bacteria or enzymes important in the biodegradation.

Combining microbiological and physico-chemical surveys is essential in efficient development and implementation of bioremediation strategies. To this end, ongoing technological as well as conceptual innovations, such as those described in this thesis, can be expected to further accelerate the importance of bioremediation as prime strategy for environmental protection in the future bio-based economy. 


\section{References}

1. Margat, J. and J. van der Gun, Groundwater around the world: A geographic synopsis. 2013.

2. Smith, M., et al., Spring - Managing groundwater sustainably. IUCN, 2016.

3. EEA, Key sources of soil contamination. E.E. Agency, 2014.

4. RIVM, Jaarverslag monitoring bodemsanering over 2009: Een rapportage van de bevoegde overheden bodemsanering. 2010: Bilthoven. p. 128.

5. EEA, Contaminants affecting soil and groundwater in Europe. E.E. Agency, 2014.

6. Foundation BE-Basic. BE-Basic. 2011 [cited 2013.

7. $\quad$ EEA, Share of renewable energy in transport. E.E. Agency, 2016.

8. Peijnenburg, W. and H.d. Hollander, Short overview of the toxicity and (modelled) degradability of model substances of the 1st generation biofuels. 2009, National Institute for Public Health and the Environment. p. 19.

9. EC. Biofuels. [cited 2018 20-02-18]; Available from: https://ec.europa.eu/energy/en/topics/renewable-energy/biofuels.

10. Stupp, D., et al., Gasoline ether oxygenate occurence in Europe, and a review of their fate and transport characteristics in the environment, in CONCAWE. 2012.

11. Chen, K.-F., Y.-C. Chang, and S.-C. Huang, Biodegradation potential of MTBE and BTEX under aerobic, nitrate reducing, and methanogenic conditions at a gasoline-contaminated site. Desalin Water Treat, 2012. 48(1-3): p. 278-284.

12. Le Digabel, Y., et al., Ethyl tert-butyl ether (ETBE) biodegradation by a syntrophic association of Rhodococcus sp. IFP 2042 and Bradyrhizobium sp. IFP 2049 isolated from a polluted aquifer. Appl Microbial Biotechnol, 2013. 97(24): p. 10531-9.

13. van Wezel, A., et al., Odour and flavour thresholds of gasoline additives (MTBE, ETBE and TAME) and their occurrence in Dutch drinking water collection areas. Chemosphere, 2009. 76(5): p. 672-6.

14. Deeb, R.A., et al., MTBE and other oxygenates: environmental sources, analysis, occurrence, and treatment. Environ Eng Sci, 2003. 20(5): p. 433-447.

15. PetrochemicalsEurope. Capacity and production data. 2017 [cited 2017.

16. Schmidt, T.C., et al., Microbial degradation of methyl tert-butyl ether and tertbutyl alcohol in the subsurface. J Contam Hydrol, 2004. 70(3-4): p. 173-203.

17. Krop, H.B., et al., n-Octanol-water partition coefficients, aqueous solubilities and Henry's law constants of fatty acid esters. Chemosphere, 1997. 34(1): p. 107-119.

18. Grbić-Galić, D., Methanogenic transformation of aromatic hydrocarbons and phenols in groundwater aquifers. Geomicrobiol J, 1990. 8(3-4): p. 167-200.

19. Häggblom, M.M., et al., Anaerobic biodegradation of Methyl tert-Butyl Ether (MTBE) and related fuel oxygenates, in Adv Appl Microbiol, S.S. Allen I. Laskin and M.G. Geoffrey, Editors. 2007, Academic Press. p. 1-20.

20. Somsamak, P., R.M. Cowan, and M.M. Häggblom, Anaerobic biotransformation of fuel oxygenates under sulfate-reducing conditions. FEMS Microbiol Ecol, 2001. 37(3): p. 259-264.

21. Yeh, C.K. and J.T. Novak, Anaerobic biodegradation of gasoline oxygenates in soils. Water Environ Res, 1994. 66(5): p. 744-752. 
22. Bombach, P., et al., Evaluation of ethyl tert-butyl ether biodegradation in a contaminated aquifer by compound-specific isotope analysis and in situ microcosms. J Hazard Mater, 2015. 286: p. 100-106.

23. Liu, T., et al., Identification of a Ruminococcaceae Species as the Methyl tertButyl Ether (MTBE) Degrading Bacterium in a Methanogenic Consortium. Environ Sci Technol, 2016. 50(3): p. 1455-1464.

24. Sun, W., X. Sun, and A.M. Cupples, Anaerobic methyl tert-butyl ether-degrading microorganisms identified in wastewater treatment plant samples by stable isotope probing. Appl Environ Microbiol, 2012. 78(8): p. 2973-80.

25. Youngster, L.K., et al., Assessment of MTBE biodegradation pathways by twodimensional isotope analysis in mixed bacterial consortia under different redox conditions. Appl Microbiol Biotechnol, 2010. 88(1): p. 309-17.

26. Waul, C., E. Arvin, and J.E. Schmidt, Long term studies on the anaerobic biodegradability of MTBE and other gasoline ethers. J Hazard Mater, 2009. 163(1): p. 427-32.

27. Wei, N. and K.T. Finneran, Microbial community analyses of three distinct, liquid cultures that degrade methyl tert-butyl ether using anaerobic metabolism. Biodegradation, 2009. 20(5): p. 695-707.

28. Fischer, A., et al., Biotic and abiotic transformations of Methyl Tertiary Butyl Ether (MTBE). Environ Sci Pollut Res, 2005. 12(6): p. 381-386.

29. Pruden, A., et al., Anaerobic biodegradation of Methyl tert-Butyl Ether under iron-reducing conditions in batch and continuous-flow cultures. Water Environ Res, 2005. 77(3): p. 297-303.

30. Bradley, P.M., F.H. Chapelle, and J.E. Landmeyer, Effect of redox conditions on MtBE biodegradation in surface water sediments. Environ Sci Technol, 2001. 35(23): p. 4643-4647.

31. Bradley, P.M., F.H. Chapelle, and J.E. Landmeyer, Methyl t-Butyl ether mineralization in surface-water sediment microcosms under denitrifying conditions. Appl Environ Microbiol, 2001. 67(4): p. 1975-1978.

32. Finneran, K.T. and D.R. Lovley, Anaerobic degradation of methyl tert-Butyl ether (MTBE) and tert-butyl alcohol (TBA). Environ Sci Technol, 2001. 35(9): p. 17851790.

33. Bradley, P.M., J.E. Landmeyer, and F.H. Chapelle, Aerobic mineralization of $M T B E$ and tert-butyl alcohol by stream-bed sediment microorganisms. Environ Sci Technol, 1999. 33(11): p. 1877-1879.

34. Mormile, M.R., S. Liu, and J.M. Suflita, Anaerobic biodegradation of gasoline oxygenates: Extrapolation of information to multiple sites and redox conditions. Environ Sci Technol, 1994. 28(9): p. 1727-1732.

35. Wei, N. and K.T. Finneran, Microbial community composition during anaerobic mineralization of tert-Butyl Alcohol (TBA) in fuel-contaminated aquifer material. Environ Sci Technol, 2011. 45(7): p. 3012-3018.

36. Puig-Grajales, L., et al., Anaerobic biodegradability of alkylphenols and fuel oxygenates in the presence of alternative electron acceptors. Appl Microbiol Biotechnol, 2000. 54(5): p. 692-697.

37. Bradley, P.M., J.E. Landmeyer, and F.H. Chapelle, TBA biodegradation in surface-water sediments under aerobic and anaerobic conditions. Environ Sci Technol, 2002. 36(19): p. 4087-4090. 
38. Day, M.J. and T. Gulliver, Rate of natural attenuation of tert-butyl alcohol at a chemical plant. Soil Sediment Contam, 2003. 12(1): p. 119-138.

39. Burland, S.M. and E.A. Edwards, Anaerobic benzene biodegradation linked to nitrate reduction. Appl Environ Microbiol, 1999. 65(2): p. 529-533.

40. Dou, J., et al., Anaerobic BTEX biodegradation linked to nitrate and sulfate reduction. J Hazard Mater, 2008. 151(2-3): p. 720-9.

41. Ulrich, A.C. and E.A. Edwards, Physiological and molecular characterization of anaerobic benzene-degrading mixed cultures. Environ Microbiol, 2003. 5(2): p. 92-102.

42. Dou, J., X. Liu, and Z. Hu, Anaerobic BTEX degradation in soil bioaugmented with mixed consortia under nitrate reducing conditions. J Environ Sci, 2008. 20(5): p. 585-592.

43. van der Zaan, B.M., et al., Anaerobic benzene degradation under denitrifying conditions: Peptococcaceae as dominant benzene degraders and evidence for a syntrophic process. Environ Microbiol, 2012. 14(5): p. 1171-81.

44. Kasai, Y., et al., RNA-based stable isotope probing and isolation of anaerobic benzene-degrading bacteria from gasoline-contaminated groundwater. Appl Environ Microbiol, 2006. 72(5): p. 3586-3592.

45. Dou, J., et al., Anaerobic benzene biodegradation by a pure bacterial culture of Bacillus cereus under nitrate reducing conditions. J Environ Sci, 2010. 22(5): p. 709-715.

46. Coates, J.D., et al., Anaerobic benzene oxidation coupled to nitrate reduction in pure culture by two strains of Dechloromonas. Nature, 2001. 411(6841): p. 10391043.

47. Oosterkamp, M.J., Physiology and biochemistry of aromatic hydrocarbondegrading bacteria that use chlorate and/or nitrate as electron acceptor. 2013, Wageningen University: Wageningen. p. 191.

48. Weelink, S., Degradation of benzene and other aromatic hydrocarbons by anaerobic bacteria, in Microbiology. 2008, Wageningen University: Wageningen.

49. Weelink, S., et al., Isolation and characterization of Alicycliphilus denitrificans Strain BC, which Grows on benzene with chlorate as the electron acceptor. Appl Environ Microbiol, 2008. 74(21): p. 6672-6681.

50. Kunapuli, U., T. Lueders, and R.U. Meckenstock, The use of stable isotope probing to identify key iron-reducing microorganisms involved in anaerobic benzene degradation. ISME J, 2007. 1(7): p. 643-653.

51. Meckenstock, R.U., et al., Anaerobic degradation of benzene and polycyclic aromatic hydrocarbons. J Mol Microbiol Biotechnol, 2016. 26(1-3): p. 92-118.

52. Vogt, C., S. Kleinsteuber, and H.H. Richnow, Anaerobic benzene degradation by bacteria. Microb Biotechnol, 2011. 4(6): p. 710-24.

53. Langenhoff, A.A.M., H. Richnow, and J. Gerritse, Benzene degradation at a site amended with nitrate or chlorate. Bioremediat J, 2009. 13(4): p. 180-187.

54. Tan, N.C., et al., Benzene degradation coupled with chlorate reduction in a soil column study. Biodegradation, 2006. 17(2): p. 113-9.

55. Chakraborty, R., et al., Anaerobic degradation of benzene, toluene, ethylbenzene, and xylene compounds by Dechloromonas Strain RCB. Appl Environ Microbiol, 2005. 71(12): p. 8649-8655.

56. Keller, A.H., S. Kleinsteuber, and C. Vogt, Anaerobic benzene mineralization by nitrate-reducing and sulfate-reducing microbial consortia enriched from the same 
site: Comparison of community composition and degradation characteristics. Microb Ecol, 2017: p. 1-13.

57. van der Waals, M.J., et al., Benzene degradation in a denitrifying biofilm reactor: activity and microbial community composition. Appl Microbial Biotechnol, 2017: p. 1-14.

58. Liou, J.S.C., C.M. DeRito, and E.L. Madsen, Field-based and laboratory stable isotope probing surveys of the identities of both aerobic and anaerobic benzenemetabolizing microorganisms in freshwater sediment. Environ Microbiol, 2008. 10(8): p. 1964-1977.

59. Kasai, Y., et al., Degradative capacities and bioaugmentation potential of an anaerobic benzene-degrading bacterium Strain DN11. Environ Sci Technol, 2007. 41(17): p. 6222-6227.

60. Ulrich, A.C., H.R. Beller, and E.A. Edwards, Metabolites detected during biodegradation of ${ }^{13} C_{6}$-benzene in nitrate-reducing and methanogenic enrichment cultures. Environ Sci Technol, 2005. 39(17): p. 6681-6691.

61. Nales, M., B.J. Butler, and E.A. Edwards, Anaerobic Benzene Biodegradation: A Microcosm Survey. Bioremediation Journal, 1998. 2(2): p. 125-144.

62. Morgan, P., S.T. Lewis, and R.J. Watkinson, Biodegradation of benzene, toluene, ethylbenzene and xylenes in gas-condensate-contaminated ground-water. Environ Pollut, 1993. 82(2): p. 181-190.

63. Villatoro-Monzon, W.R., A.M. Mesta-Howard, and E. Razo-Flores, Anaerobic biodegradation of BTEX using $\mathrm{Mn}(\mathrm{IV})$ and $\mathrm{Fe}(\mathrm{III})$ as alternative electron acceptors. Water Sci Technol, 2003. 48(6): p. 125-31.

64. Zhang, T., et al., Anaerobic benzene oxidation via phenol in Geobacter metallireducens. Appl Environ Microbiol, 2013. 79(24): p. 7800-7806.

65. Zhang, T., et al., Anaerobic benzene oxidation by Geobacter species. Appl Environ Microbiol, 2012. 78(23): p. 8304-8310.

66. Kunapuli, U., et al., Identification of intermediates formed during anaerobic benzene degradation by an iron-reducing enrichment culture. Environ Microbiol, 2008. 10(7): p. 1703-1712.

67. Jahn, M.K., S.B. Haderlein, and R.U. Meckenstock, Anaerobic degradation of benzene, toluene, ethylbenzene, and o-xylene in sediment-free iron-reducing enrichment cultures. ApplEnviron Microbiol, 2005. 71(6): p. 3355-3358.

68. Berlendis, S., et al., Anaerobic biodegradation of BTEX by original bacterial communities from an underground gas storage aquifer. Environ Sci Technol, 2010. 44(9): p. 3621-3628.

69. Herrmann, S., et al., Functional characterization of an anaerobic benzenedegrading enrichment culture by DNA stable isotope probing. Environ Microbiol, 2010. 12(2): p. 401-411.

70. Abu Laban, N., et al., Anaerobic benzene degradation by gram-positive sulfatereducing bacteria. FEMS Microbiol Ecol, 2009. 68(3): p. 300-311.

71. Herrmann, S., et al., Enrichment of anaerobic benzene-degrading microorganisms by in situ microcosms. FEMS Microbiol Ecol, 2008. 63(1): p. 94-106.

72. Jin, S., et al., Bioremediation of benzene, ethylbenzene, and xylenes in groundwater under iron-amended, sulfate-reducing conditions. EnvironToxicol Chem, 2007. 26(2): p. 249-253.

73. Phelps, C.D. and L.Y. Young, Anaerobic biodegradation of BTEX and gasoline in various aquatic sediments. Biodegradation, 1999. 10(1): p. 15-25. 
74. Weiner, J.M. and D.R. Lovley, Anaerobic benzene degradation in petroleumcontaminated aquifer sediments after inoculation with a benzene-oxidizing enrichment. Appl Environ Microbiol, 1998. 64(2): p. 775-778.

75. Kazumi, J., et al., Anaerobic degradation of benzene in diverse anoxic anvironments. Environ Sci Technol, 1997. 31(3): p. 813-818.

76. Phelps, C.D., J. Kazumi, and L.Y. Young, Anaerobic degradation of benzene in BTX mixtures dependent on sulfate reduction. FEMS Microbiol Lett, 1996. 145(3): p. 433-437.

77. Edwards, E.A. and D. Grbić-Galić, Complete mineralization of benzene by aquifer microorganisms under strictly anaerobic conditions. Appl Environ Microbiol, 1992. 58(8): p. 2663-2666.

78. Kleinsteuber, S., et al., Molecular characterization of bacterial communities mineralizing benzene under sulfate-reducing conditions. FEMS Microbiol Ecol, 2008. 66(1): p. 143-157.

79. Liang, X., et al., Anaerobic conversion of chlorobenzene and benzene to CH4 and CO2 in bioaugmented microcosms. Environ Sci Technol, 2013. 47(5): p. 23782385.

80. Masumoto, H., et al., Complete mineralization of benzene by a methanogenic enrichment culture and effect of putative metabolites on the degradation. Chemosphere, 2012. 86(8): p. 822-828.

81. Chang, W., Y. Um, and T.R.P. Holoman, Molecular characterization of anaerobic microbial communities from benzene-degrading sediments under methanogenic conditions. Biotechnol Prog, 2005. 21(6): p. 1789-1794.

82. Cervantes, F.J., et al., Anaerobic degradation of benzene by enriched consortia with humic acids as terminal electron acceptors. J Hazard Mater, 2011. 195(Supplement C): p. 201-207.

83. Dou, J., X. Liu, and Z. Hu, Substrate interactions during anaerobic biodegradation of BTEX by the mixed cultures under nitrate reducing conditions. $\mathrm{J}$ Hazard Mater, 2008. 158(2-3): p. 264-72.

84. Dalton, H. and D.I. Stirling, Co-metabolism. Philos Trans R Soc Lond B Biol Sci, 1982. 297(1088): p. 481-96.

85. Wilson, J.T., et al., Effect of biofuels on biodegradation of benzene and toluene at gasoline spill sites. Ground Water Monit Remediat, 2016. 36(4): p. 50-61.

86. Da Silva, M.L.B. and P.J.J. Alvarez, Effects of ethanol versus MTBE on benzene, toluene, ethylbenzene, and xylene natural attenuation in aquifer columns. $\mathrm{J}$ Environ Eng, 2002. 128(9): p. 862-867.

87. Ruiz-Aguilar, G.M.L., et al., Effect of ethanol and methyl-Tert-butyl ether on monoaromatic hydrocarbon biodegradation: Response variability for different aquifer materials under various electron-accepting conditions. Environ Toxicol Chem, 2002. 21(12): p. 2631-2639.

88. Youngster, L.K.G., P. Somsamak, and M.M. Häggblom, Effects of co-substrates and inhibitors on the anaerobic O-demethylation of methyl tert-butyl ether (MTBE). Appl Microbiol Biotechnol, 2008. 80(6): p. 1113-1120.

89. Mulligan, C. An overview of in situ bioremediation processes. in Proceedings of the 29th Annual Conference of the Canadian Society for Civil Engineering, Victoria, BC, May. 2001.

90. Farhadian, M., et al., In situ bioremediation of monoaromatic pollutants in groundwater: A review. Bioresour Technol, 2008. 99(13): p. 5296-5308. 
91. Azubuike, C.C., C.B. Chikere, and G.C. Okpokwasili, Bioremediation techniquesclassification based on site of application: principles, advantages, limitations and prospects. World J Microbiol Biotechnol, 2016. 32(11): p. 180.

92. Karigar, C.S. and S.S. Rao, Role of microbial enzymes in the bioremediation of pollutants: A review. Enzyme Res, 2011. 2011: p. 11.

93. EEA, Most frequently applied remediation techniques for contaminated soil. E.E. Agency, 2014.

94. Adams, G.O., et al., Bioremediation, biostimulation and bioaugmention: A review. Int J Environ Bioremediat Biodegrad, 2015. 3(1): p. 28-39.

95. Menendez-Vega, D., et al., Engineered in situ bioremediation of soil and groundwater polluted with weathered hydrocarbons. Eur J Soil Biol, 2007. 43(56): p. 310-321.

96. Tsai, T.T., et al., Application of surfactant enhanced permanganate oxidation and bidegradation of trichloroethylene in groundwater. J Hazard Mater, 2009. 161(1): p. 111-119.

97. Gentry, T., C. Rensing, and I.A.N. Pepper, New approaches for bioaugmentation as a remediation technology. Crit Rev Environ Sci Technol, 2004. 34(5): p. 447494.

98. El Fantroussi, S. and S.N. Agathos, Is bioaugmentation a feasible strategy for pollutant removal and site remediation? Curr Opin Microbiol, 2005. 8(3): p. 268275.

99. Da Silva, M.L.B. and P.J.J. Alvarez, Enhanced anaerobic biodegradation of benzene-toluene-ethylbenzene-xylene-ethanol mixtures in bioaugmented aquifer columns. Appl Environ Microbiol, 2004. 70(8): p. 4720-4726.

100. Fennell, D.E., et al., 6.13 - Dehalogenation of polychlorinated dibenzo-p-dioxins and dibenzofurans, polychlorinated biphenyls, and brominated flame retardants, and potential as a bioremediation strategy, in Comprehensive Biotechnology (Second Edition), M. Moo-Young, Editor. 2011, Academic Press: Burlington. p. 135-149.

101. Lin, B., et al., Phylogenetic and physiological diversity of dissimilatory ferric iron reducers in sediments of the polluted Scheldt estuary, Northwest Europe. Environ Microbiol, 2007. 9(8): p. 1956-68.

102. Stewart, E.J., Growing Unculturable Bacteria. J Bacteriol, 2012. 194(16): p. 41514160.

103. Nichols, D., et al., Use of Ichip for high-throughput in situ cultivation of "uncultivable" microbial species. Appl Environ Microbiol, 2010. 76(8): p. 24452450.

104. Megharaj, M., et al., Superior survival and degradation of dibenzo-p-dioxin and dibenzofuran in soil by soil-adapted Sphingomonas sp. strain RW1. Appl Microbiol Biotechnol, 1997. 48(1): p. 109-114.

105. Nam, I.-H., et al., Biological removal of polychlorinated dibenzo-p-dioxins from incinerator fly ash by Sphingomonas wittichii RW1. Water Res, 2005. 39(19): p. 4651-4660.

106. Cullum, A.J., A.F. Bennett, and R.E. Lenski, Evolutionary Adaptation to Temperature. IX. Preadaptation to Novel Stressful Environments of Escherichia coli Adapted to High Temperature. Evolution, 2001. 55(11): p. 2194-2202.

107. Ketola, T., et al., Fluctuating temperature leads to evolution of thermal generalism and preadaptation to novel environments. Evolution, 2013. 67(10): p. 2936-2944. 
108. McMurdie, P.J., et al., Localized Plasticity in the Streamlined Genomes of Vinyl Chloride Respiring Dehalococcoides. PLoS Genet, 2009. 5(11): p. e1000714.

109. van der Meer, J.R., et al., Evolution of a pathway for chlorobenzene metabolism leads to natural attenuation in contaminated groundwater. Appl Environ Microbiol, 1998. 64(11): p. 4185-4193.

110. Vogel, T.M., Bioaugmentation as a soil bioremediation approach. Curr Opin Biotechnol, 1996. 7(3): p. 311-316.

111. Suarez, M.P. and H.S. Rifai, Biodegradation rates for fuel hydrocarbons and chlorinated solvents in groundwater. Bioremediat J, 1999. 3(4): p. 337-362.

112. Lovley, D.R., Potential for anaerobic bioremediation of BTEX in petroleumcontaminated aquifers. J Ind Microbiol Biotechnol, 1997. 18(2-3): p. 75-81.

113. Salinero, K.K., et al., Metabolic analysis of the soil microbe Dechloromonas aromatica str. RCB: indications of a surprisingly complex life-style and cryptic anaerobic pathways for aromatic degradation. BMC Genomics, 2009. 10: p. 351351.

114. Luo, F., et al., Metatranscriptome of an anaerobic benzene-degrading, nitratereducing enrichment culture reveals involvement of carboxylation in benzene ring activation. Appl Environ Microbiol, 2014. 80(14): p. 4095-4107.

115. Abu Laban, N., et al., Identification of enzymes involved in anaerobic benzene degradation by a strictly anaerobic iron-reducing enrichment culture. Environ Microbiol, 2010. 12(10): p. 2783-2796.

116. Taubert, M., et al., Protein-SIP enables time-resolved analysis of the carbon flux in a sulfate-reducing, benzene-degrading microbial consortium. ISME J, 2012. 6(12): p. 2291-2301.

117. Winderl, C., et al., Depth-resolved quantification of anaerobic toluene degraders and aquifer microbial community patterns in distinct redox zones of a tar oil contaminant Plume. Appl Environ Microbiol, 2008. 74(3): p. 792-801.

118. Ruan, M.-Y., et al., Molecular diversity of bacterial bamA gene involved in anaerobic degradation of aromatic hydrocarbons in mesophilic petroleum reservoirs. Int Biodeterior Biodegradation, 2016. 114: p. 122-128.

119. Lueders, T., The ecology of anaerobic degraders of BTEX hydrocarbons in aquifers. FEMS Microbiol Ecol, 2017. 93(1).

120. Ercan, O., et al., Physiological and transcriptional responses of different industrial microbes at near-zero specific growth rates. Appl Environ Microbiol, 2015. 81(17): p. 5662-5670.

121. Van Verseveld, H.W., et al., Modeling of microbial substrate conversion, growth and product formation in a recycling fermentor. Antonie van Leeuwenhoek, 1986. 52(4): p. 325-342.

122. Helmus, R., et al. Identification strategy for transformation products of environmental pollutants using UHPLC-QTOF. in Nontarget 2016. 2016. Ascona.

123. Lowry, O.H., et al., Protein measurement with the folin phenol reagent. J Biol Chem, 1951. 193(1): p. 265-275.

124. Rajeev, L., et al., Dynamic cyanobacterial response to hydration and dehydration in a desert biological soil crust. ISME J, 2013. 7(11): p. 2178-2191.

125. Atashgahi, S., et al., Geochemical and microbial community determinants of reductive dechlorination at a site biostimulated with glycerol. Environ Microbiol, 2016. 
126. Tian, L., et al., Effects of pectin supplementation on the fermentation patterns of different structural carbohydrates in rats. Mol Nutr Food Res, 2016: p. n/a-n/a.

127. Ramiro-Garcia, J., et al., NG-Tax, a highly accurate and validated pipeline for analysis of $16 \mathrm{~S}$ rRNA amplicons from complex biomes [version 1; referees: 2 approved with reservations, 1 not approved]. F1000 Research, 2016. 5(1791).

128. Quast, C., et al., The SILVA ribosomal RNA gene database project: improved data processing and web-based tools. Nucl Acids Res, 2013. 41(D1): p. D590-D596.

129. Caporaso, J.G., et al., QIIME allows analysis of high-throughput community sequencing data. Nat Methods, 2010. 7(5): p. 335-6.

130. Beller, H.R., et al., A real-time polymerase chain reaction method for monitoring anaerobic, hydrocarbon-degrading bacteria based on a catabolic gene. Environ Sci Technol, 2002. 36(18): p. 3977-3984.

131. Lane, D.J., 16S/23S rRNA sequencing. Nucleic acid techniques in bacterial systematics, 1991: p. 115-175.

132. Muyzer, G. and N.B. Ramsing, Molecular methods to study the organization of microbial communities. Water Sci Technol, 1995. 32(8): p. 1-9.

133. Staats, M., M. Braster, and W.F.M. Röling, Molecular diversity and distribution of aromatic hydrocarbon-degrading anaerobes across a landfill leachate plume. Environ Microbiol, 2011. 13(5): p. 1216-1227.

134. Koressaar, T. and M. Remm, Enhancements and modifications of primer design program Primer3. Bioinformatics, 2007. 23(10): p. 1289-1291.

135. Untergasser, A., et al., Primer3-new capabilities and interfaces. Nucl Acids Res, 2012. 40(15): p. e115.

136. Ye, J., et al., Primer-BLAST: a tool to design target-specific primers for polymerase chain reaction. BMC Bioinformatics, 2012. 13: p. 134.

137. Stothard, P., The sequence manipulation suite: JavaScript programs for analyzing and formatting protein and DNA sequences. Biotechniques, 2000. 28(6): p. 1102, 1104.

138. da Rocha, U.N., J.D. van Elsas, and L.S. van Overbeek, Real-time PCR detection of Holophagae (Acidobacteria) and Verrucomicrobia subdivision 1 groups in bulk and leek (Allium porrum) rhizosphere soils. J Microbiol Methods, 2010. 83(2): p. 141-148.

139. Milo, R., What is the total number of protein molecules per cell volume? A call to rethink some published values. BioEssays, 2013. 35(12): p. 1050-1055.

140. Strous, M., et al., The sequencing batch reactor as a powerful tool for the study of slowly growing anaerobic ammonium-oxidizing microorganisms. Appl Microbiol Biotechnol, 1998. 50(5): p. 589-596.

141. Rittmann, B.E. and P.L. McCarty, Model of steady-state-biofilm kinetics. Biotechnol Bioeng, 1980. 22(11): p. 2343-2357.

142. Nielsen, P.H., Biofilm dynamics and kinetics during high-rate sulfate reduction under anaerobic conditions. Appl Environ Microbiol, 1987. 53(1): p. 27-32.

143. Faith, D.P., The role of the phylogenetic diversity measure, PD, in bio-informatics: Getting the definition right. Evol Bioinform Online, 2006. 2: p. 277-283.

144. Liang, B., et al., Anaerolineaceae and Methanosaeta turned to be the dominant microorganisms in alkanes-dependent methanogenic culture after long-term of incubation. AMB Express, 2015. 5: p. 37.

145. Sutton, N.B., et al., Impact of long-term diesel contamination on soil microbial community structure. Appl Environ Microbiol, 2013. 79(2): p. 619-630. 
146. Yamada, T., et al., Anaerolinea thermolimosa sp. nov., Levilinea saccharolytica gen. nov., sp. nov. and Leptolinea tardivitalis gen. nov., sp. nov., novel filamentous anaerobes, and description of the new classes Anaerolineae classis nov. and Caldilineae classis nov. in the bacterial phylum Chloroflexi. Int J Syst Evol Microbiol, 2006. 56(6): p. 1331-1340.

147. Sekiguchi, Y., et al., Anaerolinea thermophila gen. nov., sp. nov. and Caldilinea aerophila gen. nov., sp. nov., novel filamentous thermophiles that represent a previously uncultured lineage of the domain Bacteria at the subphylum level. Int J Syst Evol Microbiol, 2003. 53(6): p. 1843-1851.

148. Rosenkranz, F., et al., Relationship between phenol degradation efficiency and microbial community structure in an anaerobic SBR. Water Res, 2013. 47(17): p. 6739-6749.

149. Kümmel, S., et al., Anaerobic naphthalene degradation by sulfate-reducing Desulfobacteraceae from various anoxic aquifers. FEMS Microbiol Ecol, 2015. 91(3).

150. Chakraborty, R. and J.D. Coates, Hydroxylation and Carboxylation-Two Crucial Steps of Anaerobic Benzene Degradation by Dechloromonas Strain RCB. Appl Environ Microbiol, 2005. 71(9): p. 5427-5432.

151. Arai, H., et al., Adaptation of Comamonas testosteroni TA441 to utilize phenol: organization and regulation of the genes involved in phenol degradation. Microbiology, 1998. 144(10): p. 2895-2903.

152. Sun, W. and A.M. Cupples, Diversity of five anaerobic toluene-degrading microbial communities investigated using stable isotope probing. Appl Environ Microbiol, 2012. 78(4): p. 972-980.

153. Schut, F., et al., Isolation of typical marine bacteria by dilution culture: Growth, maintenance, and characteristics of isolates under laboratory conditions. Appl Environ Microbiol, 1993. 59(7): p. 2150-2160.

154. Kjelleberg, S., et al., How do non-differentiating bacteria adapt to starvation? Antonie van Leeuwenhoek, 1993. 63(3): p. 333-341.

155. Gottschal, J.C., Growth kinetics and competition - some contemporary comments. Antonie van Leeuwenhoek, 1993. 63(3): p. 299-313.

156. Hyman, M., Biodegradation of gasoline ether oxygenates. Curr Opin Biotechnol, 2013. 24(3): p. 443-450.

157. Suflita, J.M. and M.R. Mormile, Anaerobic biodegradation of known and potential gasoline oxygenates in the terrestrial subsurface. Environ Sci Technol, 1993. 27(5): p. 976-978.

158. Somsamak, P., H.H. Richnow, and M.M. Häggblom, Carbon Isotopic Fractionation during Anaerobic Biotransformation of Methyl tert-Butyl Ether and tert-Amyl Methyl Ether. Environ Sci Technol, 2005. 39(1): p. 103-109.

159. Somsamak, P., H.H. Richnow, and M.M. Häggblom, Carbon isotope fractionation during anaerobic degradation of Methyl tert-Butyl Ether under sulfate-reducing and methanogenic conditions. Appl Environ Microbiol, 2006. 72(2): p. 1157-1163.

160. Wilson, J.T., et al., Anaerobic biodegradation of MTBE at a gasoline spill site. Ground Water Monit Remediat, 2005. 25(3): p. 103-115.

161. Fiorenza, S. and H.S. Rifai, Review of MTBE biodegradation and bioremediation. Bioremediat J, 2003. 7(1): p. 1-35. 
162. Hernandez-Perez, G., F. Fayolle, and J.P. Vandecasteele, Biodegradation of ethyl t-butyl ether (ETBE), methyl t-butyl ether (MTBE) and t-amyl methyl ether (TAME) by Gordonia terrae. Appl Microbiol Biotechnol, 2001. 55(1): p. 117-21.

163. Chin, J.-Y. and S.A. Batterman, VOC composition of current motor vehicle fuels and vapors, and collinearity analyses for receptor modeling. Chemosphere, 2012. 86(9): p. 951-958.

164. Coates, J.D., R. Chakraborty, and M.J. McInerney, Anaerobic benzene biodegradation--a new era. Res Microbiol, 2002. 153(10): p. 621-8.

165. Sedran, M.A., et al., Effect of BTEX on degradation of MTBE and TBA by mixed bacterial consortium. J Environ Eng, 2002. 128(9): p. 830-835.

166. Deeb, R.A., et al., Substrate interactions in BTEX and MTBE mixtures by an MTBE-degrading isolate. Environ Sci Technol, 2001. 35(2): p. 312-317.

167. Corseuil, H.X., et al., The influence of the gasoline oxygenate ethanol on aerobic and anaerobic BTX biodegradation. Water Res, 1998. 32(7): p. 2065-2072.

168. Lovanh, N., C.S. Hunt, and P.J.J. Alvarez, Effect of ethanol on BTEX biodegradation kinetics: aerobic continuous culture experiments. Water Res, 2002. 36(15): p. 3739-3746.

169. Harbaugh, A.W., MODFLOW-2005, the U.S. geological survey modular groundwater model -- the ground-water flow process: U.S. geological survey techniques and methods 6-A16. 2005.

170. Harbaugh, A.W., et al., MODFLOW-2005 version 1.12.00, the U.S. geological survey modular groundwater model: U.S. geological survey software release, 03 February. 2017.

171. Schwertmann, U. and R.M. Cornell, Ferrihydrite, in Iron oxides in the laboratory. 2007, Wiley-VCH Verlag GmbH. p. 103-112.

172. Black, L. and D. Fine, High levels of monoaromatic compounds limit the use of solid-phase microextraction of methyl tert-butyl ether and tert-butyl alcohol. Environ Sci Technol, 2001. 35(15): p. 3190-3192.

173. Rohwerder, T., et al., The alkyl tert-butyl ether intermediate 2-hydroxyisobutyrate is degraded via a novel cobalamin-dependent mutase pathway. Appl Environ Microbiol, 2006. 72(6): p. 4128-35.

174. Youngster, L.K.G., L.J. Kerkhof, and M.M. Häggblom, Community characterization of anaerobic methyl tert-butyl ether (MTBE)-degrading enrichment cultures. FEMS Microbiol Ecol, 2010. 72(2): p. 279-288.

175. Landmeyer, J.E., et al., Fate of MTBE relative to benzene in a gasolinecontaminated aquifer (1993-98). Ground Water Monit Remediat, 1998. 18(4): p. 93-102.

176. Janssen, P.H. and B. Schink, Pathway of anaerobic poly- $\beta$-hydroxybutyrate degradation by Ilyobacter delafieldii. Biodegradation, 1993. 4(3): p. 179-185.

177. Lopes Ferreira, N., C. Malandain, and F. Fayolle-Guichard, Enzymes and genes involved in the aerobic biodegradation of methyl tert-butyl ether (MTBE). Appl Microbiol Biotechnol, 2006. 72(2): p. 252-262.

178. Sedran, M.A., et al., Biodegradation of Methyl tert-Butyl Ether and BTEX at varying hydraulic retention times. Water Environ Res, 2004. 76(1): p. 47-55.

179. Pruden, A. and M. Suidan, Effect of benzene, toluene, ethylbenzene, and p-xylene (BTEX) mixture on biodegradation of methyl tert-butyl ether (MTBE) and tertbutyl alcohol (TBA) by pure culture UC1. Biodegradation, 2004. 15(4): p. 213-27. 
180. Deshpande, S.D., T. Chakrabarti, and P.V.R. Subrahmanyam, Mixed-substrate utilization by acclimated activated sludge in batch and continuous-flow stirred tank reactors. Environ Sci Technol, 1987. 21(10): p. 1003-1008.

181. Kharoune, M., A. Pauss, and J.M. Lebeault, Aerobic biodegradation of an oxygenates mixture: ETBE, MTBE and TAME in an upflow fixed-bed reactor. Water Res, 2001. 35(7): p. 1665-1674.

182. Gunasekaran, V., L. Stam, and M. Constantí, The effect of BTX compounds on the biodegradation of ETBE by an ETBE degrading bacterial consortium. Biotechnol Bioprocess Eng, 2013. 18(6): p. 1216-1223.

183. David, F. and P. Sandra, Stir bar sorptive extraction for trace analysis. Journal of Chromatography A, 2007. 1152: p. 54-69.

184. Fayolle, F., J.-P. Vandecasteele, and F. Monot, Microbial degradation and fate in the environment of methyl tert-butyl ether and related fuel oxygenates. Appl Microbial Biotechnol, 2001. 56(3): p. 339-349.

185. Babé, A., et al., Biodegradability of oxygenates by microflora from MTBEContaminated sites: new molecular tools, in Fuel Oxygenates, D. Barceló, Editor. 2007, Springer Berlin Heidelberg: Berlin, Heidelberg. p. 75-98.

186. VROM, M.o., Application of duty for concern from soil regulation on MTBE and ETBE contamination. State Journal Nr. 2139, 2008.

187. Shih, T., et al., Evaluation of the impact of fuel hydrocarbons and oxygenates on groundwater resources. Environ Sci Technol, 2004. 38(1): p. 42-48.

188. Fayolle, F., et al., Isolation of two aerobic bacterial strains that degrade efficiently ethyl t-butyl ether (ETBE). Biotechnol Lett, 1998. 20(3): p. 283-286.

189. Kharoune, M., et al., Isolation and characterization of two aerobic bacterial strains that completely degrade ethyl tert-butyl ether (ETBE). Appl Microbial Biotechnol, 2001. 55(3): p. 348-353.

190. Chauvaux, S., et al., Cloning of a genetically unstable cytochrome P-450 gene cluster involved in degradation of the pollutant Ethyltert-Butyl Ether by Rhodococcus ruber. J Bacteriol, 2001. 183(22): p. 6551-6557.

191. Beguin, P., et al., Genes involved in the degradation of ether fuels by bacteria of the Mycobacterium/Rhodococcus Group. Oil Gas Sci Technol, 2003. 58(4): p. 489-495.

192. Malandain, C., F. Fayolle-Guichard, and T.M. Vogel, Cytochromes P450mediated degradation of fuel oxygenates by environmental isolates. FEMS Microbiol Ecol, 2010. 72(2): p. 289-296.

193. Schuster, J., et al., Constitutive expression of the cytochrome P450 EthABCD monooxygenase system enables degradation of synthetic dialkyl ethers in Aquincola tertiaricarbonis L108. Appl Environ Microbiol, 2013. 79(7): p. 23212327.

194. Ramanan, R., et al., Algae-bacteria interactions: Evolution, ecology and emerging applications. Biotechnol Adv, 2016. 34(1): p. 14-29.

195. Sinclair, J.L. and W.C. Ghiorse, Distribution of aerobic bacteria, protozoa, algae, and fungi in deep subsurface sediments. Geomicrobiol J, 1989. 7(1-2): p. 15-31.

196. Zhong, W., et al., Aerobic degradation of methyl tert-butyl ether in a closed symbiotic system containing a mixed culture of Chlorella ellipsoidea and Methylibium petroleiphilum PM1. J Hazard Mater, 2011. 185(2-3): p. 1249-1255.

197. Muñoz, R., et al., Combined carbon and nitrogen removal from acetonitrile using algal-bacterial bioreactors. Appl Microbial Biotechnol, 2005. 67(5): p. 699-707. 
198. Ryu, B.-G., et al., Algal-bacterial process for the simultaneous detoxification of thiocyanate-containing wastewater and maximized lipid production under photoautotrophic/photoheterotrophic conditions. Bioresour Technol, 2014. 162: p. 70-79.

199. Derakhshan, Z., et al., Studies on influence of process parameters on simultaneous biodegradation of atrazine and nutrients in aquatic environments by a membrane photobioreactor. Environ Res, 2018. 161: p. 599-608.

200. van der Waals, M.J., et al., Anaerobic degradation of a mixture of MtBE, EtBE, $T B A$, and benzene under different redox conditions. Appl Microbial Biotechnol, 2018. 102(7): p. 3387-3397.

201. Zehnder, A. and K. Wuhrmann, Titanium (III) citrate as a nontoxic oxidationreduction buffering system for the culture of obligate anaerobes. Science, 1976. 194(4270): p. 1165-1166.

202. Bui, T.P.N., et al., Production of butyrate from lysine and the Amadori product fructoselysine by a human gut commensal. Nat Commun, 2015. 6.

203. Di Lonardo, D.P., et al., Priming of soil organic matter: Chemical structure of added compounds is more important than the energy content. Soil Biol Biochem, 2017. 108: p. 41-54.

204. Lueders, T., M. Manefield, and M.W. Friedrich, Enhanced sensitivity of DNA- and rRNA-based stable isotope probing by fractionation and quantitative analysis of isopycnic centrifugation gradients. Environ Microbiol, 2004. 6(1): p. 73-78.

205. Vetriani, C., et al., Population structure and phylogenetic characterization of marine benthic archaea in deep-sea sediments. Appl Environ Microbiol, 1999. 65(10): p. 4375-4384.

206. Van Der Zaan, B., et al., Stability of the total and functional microbial communities in river sediment mesocosms exposed to anthropogenic disturbances. FEMS Microbiol Ecol, 2010. 74(1): p. 72-82.

207. Schmidt, R., et al., Involvement of a novel enzyme, MdpA, in methyl tert-butyl ether degradation in Methylibium petroleiphilum PM1. Appl Environ Microbiol, 2008. 74(21): p. 6631-8.

208. Brad, T., et al., Eukaryotic diversity in an anaerobic aquifer polluted with landfill leachate. Appl Environ Microbiol, 2008. 74(13): p. 3959-3968.

209. Ohkubo, N. and O. Yagi, Biodegradation of Ethyl tert-Butyl Ether (ETBE) by Rhodococcus erythropolis ET10. J Environ Biotechnol, 2012. 12(1): p. 39-46.

210. Kharoune, M., et al., Aerobic degradation of ethyl-tert-butyl ether by a microbial consortium: Selection and evaluation of biodegradation ability. Environ Toxicol Chem, 2002. 21(10): p. 2052-2058.

211. Yaneva, N., et al., Bacterial acyl-CoA mutase specifically catalyzes coenzyme B12-dependent isomerization of 2-hydroxyisobutyryl-CoA and (S)-3hydroxybutyryl-CoA. J Biol Chem, 2012. 287(19): p. 15502-11.

212. John, R.P., et al., Micro and macroalgal biomass: A renewable source for bioethanol. Bioresour Technol, 2011. 102(1): p. 186-193.

213. Rettedal, E.A. and V.S. Brözel, Characterizing the diversity of active bacteria in soil by comprehensive stable isotope probing of DNA and RNA with H(2)(18)O. MicrobiologyOpen, 2015. 4(2): p. 208-219.

214. Thomson, B.C., et al., Plant soil interactions alter carbon cycling in an upland grassland soil. Front Microbiol, 2013. 4: p. 253. 
215. Atashgahi, S., et al., A benzene-degrading nitrate-reducing microbial consortium displays aerobic and anaerobic benzene degradation pathways. Sci Rep, 2018. 8(1): p. 4490.

216. Khan, F.I., T. Husain, and R. Hejazi, An overview and analysis of site remediation technologies. J Environ Manage, 2004. 71(2): p. 95-122.

217. Heijthuijsen, J.H.F.G. and T.A. Hansen, Interspecies hydrogen transfer in cocultures of methanol-utilizing acidogens and sulfate-reducing or methanogenic bacteria. FEMS Microbiol Lett, 1986. 38(1): p. 57-64.

218. Ludwig, W., et al., ARB: a software environment for sequence data. Nucleic Acids Res, 2004. 32(4): p. 1363-71.

219. Darbre, P.D., Basic molecular biology: Essential techniques. 1999: Wiley.

220. Graupner, S., et al., Requirement of novel competence genes pilT and pilU of Pseudomonas stutzeri for natural transformation and suppression of pilT deficiency by a hexahistidine tag on the Type IV pilus protein PilAI. J Bacteriol, 2001. 183(16): p. 4694-4701.

221. Muschiol, S., et al., Uptake of extracellular DNA: Competence induced pili in natural transformation of Streptococcus pneumoniae. Bioessays, 2015. 37(4): p. 426-435.

222. Peabody, C.R., et al., Type II protein secretion and its relationship to bacterial type IV pili and archaeal flagella. Microbiology, 2003. 149(11): p. 3051-3072.

223. Nielsen, K.M., et al., Release and persistence of extracellular DNA in the environment. Environ Biosafety Res, 2007. 6(1-2): p. 37-53.

224. Syvanen, M., Horizontal gene transfer: evidence and possible consequences. Annu Rev Genet, 1994. 28: p. 237-61.

225. Chen, L.-X., et al., Comparative metagenomic and metatranscriptomic analyses of microbial communities in acid mine drainage. ISME J, 2014. 9: p. 1579.

226. Whitehead, A., Evolutionary genomics of environmental pollution, in Ecological genomics: Ecology and the evolution of genes and genomes, C.R. Landry and N. Aubin-Horth, Editors. 2014, Springer Netherlands: Dordrecht. p. 321-337.

227. Atashgahi, S., et al., Prospects for harnessing biocide resistance for bioremediation and detoxification. Science, 2018. 360(6390): p. 743-746.

228. Jensen, K.L. and R. Datta, Ethers from ethanol. 1. equilibrium thermodynamic analysis of the liquid-phase ethyl ferf-butyl ether reaction. Ind Eng Chem Res, 1995. 34(1): p. 392-399.

229. Madigan, M.T., J.M. Martinko, and J. Parker, Brock Biology of microorganisms. 2003: Prentice Hall/Pearson Education.

230. Bell, J., et al., Stripping of volatile organic compounds at full-scale municipal wastewater treatment plants. Water Environ Res, 1993. 65(6): p. 708-716.

231. Muñoz, R. and B. Guieysse, Algal-bacterial processes for the treatment of hazardous contaminants: A review. Water Res, 2006. 40(15): p. 2799-2815.

232. Fuentes, J.L., et al., Impact of microalgae-bacteria interactions on the production of algal biomass and associated compounds. Mar Drugs, 2016. 14(5): p. 100.

233. Lian, J., et al., The effect of the algal microbiome on industrial production of microalgae. Microb Biotechnol, 2018. In press.

234. Hjelm, M., et al., Selection and identification of autochthonous potential probiotic bacteria from turbot larvae (Scophthalmus maximus) rearing units. Syst Appl Microbiol, 2004. 27(3): p. 360-371. 
235. Luo, H. and M.A. Moran, Evolutionary ecology of the marine Roseobacter clade. Microbiol Mol Biol Rev, 2014. 78(4): p. 573-587.

236. Chen, C.S., et al., Assessing soil and groundwater contamination from biofuel spills. Environ Sci Process Impacts, 2015. 17(3): p. 533-542.

237. Boopathy, R., Factors limiting bioremediation technologies. Bioresour Technol, 2000. 74(1): p. 63-67.

238. Garbisu, C., et al., Plasmid-mediated bioaugmentation for the bioremediation of contaminated soils. Front Microbiol, 2017. 8: p. 1966.

239. Nicodem, D.E., et al., Photochemical processes and the environmental impact of petroleum spills. Biogeochemistry, 1997. 39(2): p. 121-138.

240. Laha, S., B. Tansel, and A. Ussawarujikulchai, Surfactant-soil interactions during surfactant-amended remediation of contaminated soils by hydrophobic organic compounds: A review. J Environ Manage, 2009. 90(1): p. 95-100.

241. Mao, X., et al., Use of surfactants for the remediation of contaminated soils: $A$ review. J Hazard Mater, 2015. 285: p. 419-435.

242. Desai, J.D. and I.M. Banat, Microbial production of surfactants and their commercial potential. Microbiol Mol Biol Rev, 1997. 61(1): p. 47-64.

243. Hunkeler, D., et al. A guide for assessing biodegradation and source identification of organic groundwater contaminants using compound specific isotope analysis (CSIA). 2009.

244. Schirmer, M. and J.F. Barker, A study of long-term MTBE attenuation in the Borden aquifer, Ontario, Canada. Groundwater Monit Remediat, 1998. 18(2): p. 113-122.

245. Butler, B.J., R. Aravena, and J.F. Barker, Monitoring Biodegradation of Methyl tert-Butyl Ether (MTBE) Using Compound-Specific Carbon Isotope Analysis. Environmental Science \& Technology, 2001. 35(4): p. 676-681.

246. Braeckevelt, M., A. Fischer, and M. Kästner, Field applicability of CompoundSpecific Isotope Analysis (CSIA) for characterization and quantification of in situ contaminant degradation in aquifers. Appl Microbiol Biotechnol, 2012. 94(6): p. 1401-1421.

247. Lu, G., et al., Natural attenuation of BTEX compounds: Model development and field-scale application. Ground Water, 1999. 37(5): p. 707-717.

248. Rolle, M., U. Maier, and P. Grathwohl, Contaminant fate and reactive transport in groundwater, in Dealing with contaminated sites: From theory towards practical application, F.A. Swartjes, Editor. 2011, Springer Netherlands: Dordrecht. p. 851 885.

249. Gomez, D.E., et al., Modeling benzene plume elongation mechanisms exerted by ethanol using RT3D with a general substrate interaction module. Water Resour Res, 2008. 44(5): p. n/a-n/a.

250. Johnston, C., et al., Bacterial transformation: Distribution, shared mechanisms and divergent control. Nat Rev Microbiol, 2014. 12(3): p. 181-196.

251. Moe-Behrens, G.H.G., R. Davis, and K.A. Haynes, Preparing synthetic biology for the world. Front Microbiol, 2013. 4: p. 5.

252. Pepper, I.L., et al., The role of cell bioaugmentation and gene bioaugmentation in the remediation of co-contaminated soils. Environ Health Perspect, 2002.

110(Suppl 6): p. 943-946.

253. Li-Beisson, Y. and G. Peltier, Third-generation biofuels: current and future research on microalgal lipid biotechnology. OCL, 2013. 20(6): p. D606. 
254. Aro, E.M., From first generation biofuels to advanced solar biofuels. Ambio, 2016. 45 Suppl 1: p. S24-31.

255. Aziz, C.E., G. Georgiou, and G.E. Speitel, Cometabolism of chlorinated solvents and binary chlorinated solvent mixtures using M. trichosporium OB3b PP358. Biotechnol Bioeng, 1999. 65(1): p. 100-107.

256. Lovley, D.R., Cleaning up with genomics: Applying molecular biology to bioremediation. Nat Rev Microbiol, 2003. 1(1): p. 35-44.

257. Srivathsan, A., et al., A MinION-based pipeline for fast and cost-effective DNA barcoding. bioRxiv, 2018.

258. Hargreaves, A.D. and J.F. Mulley, Assessing the utility of the Oxford Nanopore MinION for snake venom gland cDNA sequencing. PeerJ, 2015. 3: p. e1441.

259. Menegon, M., et al., On site DNA barcoding by nanopore sequencing. PLoS ONE, 2017. 12(10): p. e 0184741.

260. Marx, V., PCR heads into the field. Nat Methods, 2015. 12: p. 393. 


\section{Summary}

In the environment, soil and groundwater contamination often occur due to anthropogenic activity. Accidental spills, leaking storage tanks or waste disposal have polluted the surrounding environment with inorganic and organic compounds, the latter including for example aromatic and aliphatic hydrocarbons and ethers. These chemicals pose a risk to the health of the environment, animals and humans. Therefore remediation technologies are needed to clean up the environment. Bioremediation is considered cost effective, sustainable and can accelerate the natural biodegradation. This thesis describes the biodegradation of fuel components. Selected contaminants included methyl tert-butyl ether (MtBE), ethyl tert-butyl ether (EtBE), tert-butyl alcohol (TBA) and benzene. Among these contaminants, benzene is often considered as risk determining compound at fuel-contaminated locations due to its solubility and mobility in aquifers as well as its toxicity. MtBE, EtBE and TBA are gasoline ether oxygenates that are often added to fuels to increase their compressibility and efficiency of combustion.

Microbial community composition dynamics was described in an anaerobic continuous biofilm culture degrading benzene with nitrate as electron acceptor. Bacteria belonging to the family Peptococcaceae were confirmed as important anaerobic benzene degrading organisms. Members of the Anaerolineaceae were predicted to be involved in benzene degradation or benzene metabolite degradation. Benzene was degraded at a rate which was four-fold higher than reported previously for anoxic systems. Furthermore, evidence was obtained that benzene degradation proceeds through an initial carboxylation step.

The biodegradation capacity of microbial communities in groundwater from a contaminated site for a mixture of fossil-based and biobased contaminants was tested at various redox conditions by microcosm experiments. The concurrence of MtBE, EtBE, TBA and benzene may affect the microbial degradation potential for both fossil-based and biobased components. MtBE degradation in microcosm cultures was most pronounced under sulphate-reducing and iron-reducing conditions prevailing at the field site. The addition of nitrate and chlorate stimulated benzene degradation, but hindered MtBE, EtBE and TBA degradation. Overall, degradation of EtBE under different redox conditions was not found. Evidence was obtained that EtBE might be cometabolically degraded during growth on methoxylated and ethoxylated substrates. Furthermore, it was hypothesized that TBA was cometabolically degraded in the presence of MtBE, syringate, vanillate or ferulate. 
Insight is provided in the micro-oxic degradation of EtBE using an algal-bacterial culture. The oxygen produced by Chlorella and Scenedesmus species was used by the bacteria in the culture to degrade EtBE. Using both nuclear magnetic resonance spectroscopy (NMR) and gas chromatography, TBA, ethanol and $\mathrm{CO}_{2}$ were determined as metabolites in this degradation process. Stable isotope probing (SIP) with ${ }^{13} \mathrm{C}$ labelled EtBE and comparison of ${ }^{13} \mathrm{C}$ - and ${ }^{12} \mathrm{C}$-enriched DNA fractions showed Halomonadaceae, Shewanellaceae, Rhodocyclaceae, Oxalobacteraceae, Comamonadaceae, Sphingomonadaceae, Hyphomicrobiaceae, Candidatus Moranbacteria, Omnitrophica, Anaerolineaceae, Nocardiaceae, and Blastocatellaceae as predominant families in the bacterial culture, albeit without significant differences in composition between fractions.

In order to provide the necessary basis towards bioaugmentation of benzenepolluted environments, several organisms were isolated from the benzene degrading biofilm culture including Pseudomonas sp. strain BN and organisms most closely related to Bacillus cereus, Ochrobactrum anthropi, Comamonas badia and Mesorhizobium plurifarium. Furthermore, it was found that the use of the biofilm culture in bioaugmentation tests can stimulate benzene degradation. It was also suggested that the $a b c A$ gene, encoding benzene carboxylase, might be or at least has been located on a mobile element. Initial results indicate that this abcA gene might be taken up by the Pseudomonas sp. strain BN isolate, enabling it to grow on benzene.

This thesis contributes to a better understanding of the ecology and physiology of the anaerobic microorganisms involved in the anaerobic biodegradation of MtBE, EtBE, TBA and benzene. Moreover, the results indicate that the choice of the different bioremediation options, i.e. natural attenuation, biostimulation and bioaugmentation, depends on redox conditions, types of contaminants and indigenous microbial communities in the subsurface. Current developments, such as those described in this thesis, can be expected to further consolidate bioremediation as prime strategy for environmental protection in the future bio-based economy where it may not only be used for the clean-up of polluted sites, but also for the prevention of emission through recovery of potentially toxic compounds from waste streams. 


\section{Samenvatting}

Bodem- en grondwaterverontreiniging wordt vaak veroorzaakt door menselijke en industriele activiteiten. Incidentele lekkage, lekkende opslagtanks of afvalverwijdering hebben onze omgeving verontreinigd met anorganische en organische verbindingen. Voorbeelden van organische verontreinigingen zijn aromatische en alifatische koolwaterstoffen. Deze chemicaliën zijn schadelijk voor onze omgeving, dieren en mensen. Daarom is sanering van verontreinigde bodem- en watersystemen belangrijk om onze omgeving weer schoon te maken. Bioremediatie wordt gezien als een kosteneffectieve en duurzame oplossing en kan het natuurlijke afbraak proces versnellen. Dit proefschrift beschrijft de anaerobe afbraak van brandstof componenten. De geselecteerde verontreinigingen zijn methyl tert-butyl ether (MtBE), ethyl tert-butyl ether (EtBE), tert-butyl alcohol (TBA) en benzeen. Onder deze verontreinigingen wordt benzeen gezien als risico stof op verontreinigde locaties, mede door de oplosbaarheid in water en de toxiciteit. MtBE, EtBE en TBA worden vaak toegevoegd aan brandstof om de verbranding te optimaliseren.

De dynamiek in de microbiële gemeenschap van een anaerobe biofilm cultuur die continu benzeen afbreekt onder nitraat-reducerende omstandigheden is onderzocht in Hoofdstuk 2. Benzeen werd in deze studie afgebroken met een 4 keer hogere snelheid dan eerder gepubliceerd. Hiernaast werd ook bewijs gevonden dat benzeen afbraak via een initiële carboxylering stap verloopt. In deze studie werden resultaten uit eerdere onderzoeken bevestigd dat bacteriën behorend tot de Peptococcaceae familie belangrijk zijn voor de anaerobe benzeenafbraak. Daarnaast zijn aanwijzingen verkregen dat ook bacteriën binnen de familie Anaerolineaceae belangrijk zijn voor de afbraak van benzeen zelf of van metabolieten die worden gevormd tijdens de initiële afbraak.

De microbiële capaciteit voor de afbraak van een mengsel van fossiele- en biobrandstof componenten werd in Hoofdstuk 3 getest onder verschillende redox condities in laboratoriumexperimenten. Dit mengsel bevatte naast de biobrandstofadditieven EtBE en TBA ook de fossiele brandstofchemicaliën MtBE en benzeen. MtBE werd goed afgebroken onder sulfaat- en ijzer-reducerende omstandigheden. Deze redox condities waren ook belangrijk op de vervuilde locatie waar het sediment en grondwater waren verkregen die zijn gebruikt in de experimenten. De toevoeging van nitraat of chloraat stimuleerde benzeenafbraak maar remde MtBE, EtBE en TBA-afbraak. EtBE-afbraak werd in deze laboratoriumexperimenten onder geen van de geteste condities gevonden. In onze experimenten bleek 162 
dat EtBE cometabool werd afgebroken in aanwezigheid van gemethoxyleerde en geethoxyleerde substraten. Daarnaast zijn aanwijzingen verkregen dat initiële resultaten dat TBA cometabool werd afgebroken met MtBE, syringaat, vanillaat of ferulaat als substraat.

In Hoofdstuk 4 wordt inzicht gegeven in de micro-oxische EtBE afbraak met behulp van een symbiotische alg-bacterie cultuur. De zuurstof geproduceerd door de algen Chlorella en Scenedusmus werd gebruikt door de bacteriën om EtBE af te breken. Door middel van nucleaire magnetische resonantie spectroscopie (NMR) en gaschromatografie werden TBA, ethanol en $\mathrm{CO}_{2}$ als metabolieten in het afbraak proces gevonden. Opname van stabiele isotopen in het DNA van EtBE afbrekende bacteriën (SIP) is gebruikt om micro-organismen te identificeren die primair betrokken zijn bij de EtBE afbraak. Bacteriën binnen de families Halomonadaceae, Shewanellaceae, Rhodocyclaceae, Oxalobacteraceae, Comamonadaceae, Sphingomonadaceae, Hyphomicrobiaceae, Candidatus Moranbacteria, Candidatus Omnitrophica, Anaerolineaceae, Nocardiaceae, en Blastocatellaceae waren betrokken bij de EtBE-afbraak.

Verschillende organismen zijn geïsoleerd uit de benzeen afbrekende biofilmcultuur beschreven onder Hoofdstuk 2, waaronder Pseudomonas stam BN en organismen fylogenetisch verwant aan Bacillus cereus, Ochrobactrum anthropi, Comamonas badia en Mesorhizobium plurifarium (Hoofdstuk 5). Daarnaast werd gevonden dat het gebruik van biofilm materiaal van deze cultuur effectief is voor bioaugmentatie testen. Initieel bewijs suggereert dat het gen $a b c A$, dat codeert voor een sleutel enzym in de anaerobe benzeen afbraak, in een mobiel element zit. Daardoor lijkt overdracht van de gerelateerde afbraakcapaciteit mogelijk. Eerste resultaten laten zijen dat Pseudomonas stutzeri dit mobiele abcA element op kan nemen in zijn genoom, waarna benzeenafbraak geïnitieerd wordt.

Dit proefzicht geeft inzicht in de ecologie en fysiologie van anaerobe microorganismen die belangrijk zijn in de afbraak van MtBE, EtBE, TBA en benzeen. Hiernaast wordt geconcludeerd dat de keuze van sanering: natuurlijke afbraak, biostimulatie of bioaugmentatie, bepaald wordt door de redox condities, type verontreiniging en de autochtone micro-organismen in de ondergrond. Nieuwe ontwikkelingen, zoals beschreven in dit proefschrift, kunnen naar verwachting het gebruik van bioremediatie als strategie voor het schoonmaken van onze omgeving en het voorkomen van toxische emissies in een 'biobased economy' verder verstevigen. 


\section{About the author}

Johanna Marcelle van der Waals was born on the $2^{\text {nd }}$ of January 1990 in Amersfoort, The Netherlands. After completing highschool in 2008, she started with the bachelor Earth Sciences at Utrecht University. She continued with the master Earth, Surface and Water, track hydrology at Utrecht University. She wrote her master thesis at Deltares entitled 'Stripping of volatile compounds with $\mathrm{CO}_{2}$ Focus on toluene and trichloroethylene'.

After writing her master thesis she started her internship at Deltares focussing on the effects of pesticides on sediment functions. In 2013, she started her $\mathrm{PhD}$ at Deltares and the Laboratory of Microbiology in Wageningen under the supervision of Dr. Jan Gerritse (daily supervisor) and Prof. Dr. Hauke Smidt.

Her project was part of the European research Picture by Sven Menschel

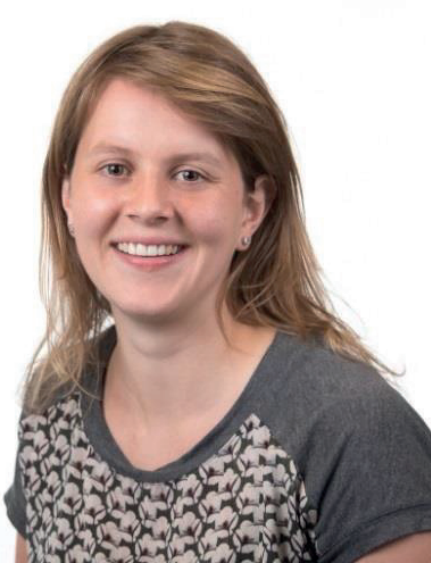
group entitled BE-Basic.

Currently she is a researcher/consultant at Deltares. 


\section{List of publications and presentations}

\section{Publications}

Atashgahi S, Hornung B, van der Waals MJ, da Rocha UN, Hugenholtz F, Nijsse B, Molenaar D, van Spanning R, Stams AJM, Gerritse J, Smidt H (2018) A benzenedegrading nitrate-reducing microbial consortium displays aerobic and anaerobic benzene degradation pathways Scientific Reports 8:4490 doi:10.1038/s41598-01822617-x

van der Waals MJ, Atashgahi S, da Rocha UN, van der Zaan BM, Smidt H, Gerritse J (2017) Benzene degradation in a denitrifying biofilm reactor: activity and microbial community composition Applied Microbiology and Biotechnology:1-14 doi:10.1007/s00253-017-8214-8

van der Waals MJ, Pijls C, Sinke AJC, Langenhoff AAM, Smidt H, Gerritse J (2018) Anaerobic degradation of a mixture of MtBE, EtBE, TBA, and benzene under different redox conditions Applied Microbiology and Biotechnology 102:33873397 doi:10.1007/s00253-018-8853-4

\section{Poster presentations}

Van der Waals, M.J., B.M. van der Zaan, U.N. Da Rocha, W.F.M. Röling, H. Smidt, J. Gerritse (2015) “Anaerobic degradation of benzene”, $\mathrm{PhD}$ trip, May 11-22, California, USA

Van der Waals, M.J., B.M. van der Zaan, U.N. Da Rocha, W.F.M. Röling, H. Smidt, J. Gerritse (2015) “Anaerobic degradation of benzene”, Annual BE-Basic symposium, March 31-April 1, Noordwijkerhout, The Netherlands

Van der Waals, M.J., B.M. van der Zaan, U.N. Da Rocha, H. Smidt, J. Gerritse (2016) "Anaerobic degradation of benzene in a biofilm reactor", CLIB-GC Retreat, February 1718, Herdecke, Germany Van der Waals, M.J., U.N. Da Rocha, S. Atashgahi, B.M. Van der Zaan, H. Smidt, J. Gerritse (2016) "Anaerobic degradation of benzene in a biofilm reactor", ECO-BIO 2016, March 6-9, Rotterdam, The Netherlands 


\section{Oral presentations}

Van der Waals, M.J. (2014) "Van de regen in de drup", Bodem Breed Symposium, November 18-19, 2014, Lunteren, The Netherlands

Van der Waals, M.J. (2015) “Anaerobic degradation of benzene”, PhD trip, May 14, 2015, Berkeley, California

Van der Waals, M.J. (2015) “Anaerobic degradation of benzene”, PhD trip, May 20, 2015, San Diego, California

Van der Waals, M.J. (2016) “Anaerobic degradation of benzene in a biofilm reactor", CLIB-GC Retreat, February 17-18, 2016, Herdecke, Germany

Van der Waals, M.J. (2016) “Anaerobic degradation of benzene in a biofilm reactor”, ECOBIO 2016, March 6-9, 2016, Rotterdam, The Netherlands

Van der Waals, M.J. (2017) “Anaerobic degradation of biofuel compounds” (pitch), FEMS microbiology, July 11, 2017, Valencia, Spain

\section{Awards}

$2^{\text {nd }}$ pitch price at FEMS 2017, Valencia, Spain 


\section{Acknowledgements - Dankwoord}

$\mathrm{Na} 4$ jaar hard werken is dan eindelijk mijn boekje af. In deze periode bij Deltares, Utrecht en het microbiologisch laboratorium te Wageningen heb ik ontzettend veel geleerd en veel nieuwe mensen leren kennen. Daarom wil ik graag een aantal mensen specifiek bedanken die mij geholpen, bijgestaan en gesteund hebben om deze mooie mijlpaal in mijn leven te behalen.

Allereerst Jan. Je was de afgelopen 4 jaar mijn dagelijkse begeleider bij Deltares. Tijdens mijn stage bij Deltares onder leiding van Bas vroeg je mij voor deze AIO positie. Na lang wikken en wegen heb ik na een eerste keer de positie af te wijzen toch besloten de positie aan te nemen en daar ben ik zeer blij mee. Ik heb ontzettend veel geleerd de afgelopen 4 jaar en daar ben ik je heel dankbaar voor. Mijn achtergrond kennis was Aardwetenschappen en daarom moest ik veel bijleren over de microbiologie. Ik heb dan ook veel inhoudelijke microbiologische kennis van je meegekregen. Daarnaast heb je mij geholpen met het creeren en bedenken van goed opgezette experimenten en het uitvoeren van gedegen onderzoek. En ja dan rest alleen nog het schrijven van artikelen, want dat was één van mijn meest gevreesde taken. Het opschrijven en ordenen van alle data en resultaten in mijn hoofd was een hele kunst, maar het is mij gelukt mooie hoofdstukken te publiceren met jouw hulp. Je hebt altijd vertrouwen in mij gehad zelfs als ik zelf weer eens dacht het niet te halen. Heel erg bedankt voor alle moeite en tijd die je hebt gestoken in mij.

Hauke, heel erg bedankt dat je mijn promotor was. Het is niet altijd makkelijk geweest om mij als "externe" AIO te helpen. Toch stond je altijd voor me klaar en met vragen kon ik snel bij je terecht. Bedankt voor je toewijding aan de promotor taken en het kritisch lezen van mijn manuscripten.

Dan zijn er nog een aantal mensen van Deltares die ik specifiek wil bedanken voor hun bijdrage.

Fredericke, als collega bij Deltares heb ik altijd heel veel microbiologische hulp van je gehad. Je hebt mij nieuwe technieken uitgelegd, geholpen met data analyses en het uitvoeren van technieken. Daarnaast was het altijd een leuke afleiding om met jou te grappen (over het foute uur?) of dingen buiten werk om te bespreken. 
Jasperien, heel erg bedankt dat je mij altijd weer kon opbeuren als ik het even niet meer helder zag. Onze etentjes waren altijd een leuke afleiding en ik hoop deze nog lang voort te zetten. Ook waren de theetjes die ik bij je kwam drinken op kantoor als ik even wilde bijpraten erg fijn.

Bas, hartelijk dank voor je gesprekken over het externe AIO zijn en moeten schipperen tussen Wageningen en Deltares. Daarnaast ben ik ook erg blij dat Jasperien en jij mijn paranimfen wilden zijn. Bedankt voor jullie hulp bij het regelen van mijn verdediging en feest:-

Erik, bij jou kon ik met al mijn uiteenlopende technische vragen terecht en wat was ik altijd blij als je ze weer beantwoordde of me kwam helpen.

Ook wil ik graag Rob, André en Piet bedanken. Jullie hulp over de afgelopen jaren was echt geweldig.

Daniel, as office buddy I would like to thank you for all the comic conversations we had. It was a pleasure to sit on the opposite site of your desk.

De afgelopen 4 jaar heb ik de mogelijkheid gehad met mensen te publiceren buiten Deltares en Wageningen. Ik wil alle coauteurs hartelijk bedanken voor alle moeite en energie die jullie in de manuscripten hebben gestoken. Met name Marion en Frans wil ik graag bedanken. Ik mocht gebruik maken van de faciliteiten binnen het NIOO en Rijkswaterstaat en daar ben ik jullie erg dankbaar voor. Naast het gebruik van deze faciliteiten hebben jullie mij ook veel bijgebracht over verschillende onderwerpen zoals SIP en algen, dank hiervoor!

Daarnaast wil ik alle mensen bij microbiologie in Wageningen bedanken. Omdat ik niet altijd aanwezig was in Wageningen was het moeilijk om vrienden te maken en in contact te komen. Toch had ik al heel snel contact met veel van jullie over werk en privé dingen. Ik wil jullie allemaal bedanken voor de leuke tijd die ik gehad heb. Naast ons werk hebben we veel leuke uitjes en feestjes gehad waarbij ik ook altijd was uitgenodigd. Dank jullie wel! Specifiek wil ik graag Romy en Teunke bedanken. Op onze AIO-reis naar Amerika was ik kamergenoten met jullie. Ik heb de USA roomies uitjes met of zonder onze mannen altijd een goede afleiding gevonden. 
Tijdens mijn promotieonderzoek heb ik meerdere studenten mogen begeleiden voor hun stage/afstuderen. Ik heb veel geleerd van deze samenwerkingen. Hartelijk bedankt voor al jullie hulp! Dank jullie wel: Isabel, Wesley en Stef.

Daarnaast wil ik graag mijn vrienden, vriendinnen en (schoon)familie bedanken. Hartelijk bedankt voor de leuke etentjes, uitjes en activiteiten buiten werk om. Ik heb hier veel positieve energie uitgehaald.

Speciaal wil ik mijn ouders en zusje bedanken. Ook al wisten jullie niet altijd waar ik het over had, jullie deden altijd je best mijn onderzoek en experimenten te snappen. Ik stel het zeer op prijs dat jullie mij altijd mijn eigen keuzes hebben laten maken en geleerd hebben vertrouwen in jezelf te hebben. Door jullie ben ik zover gekomen in mijn leven.

Lieve Kees, als allerlaatste wil ik jou bedanken. Bedankt voor je geduld met mij. Jij gaf mij altijd het gevoel dat ik moest vertrouwen op mezelf en meer uit mezelf kon halen. Als ik ergens mee zat of helemaal klaar was met het schrijven van mijn proefschrift, beurde jij me altijd weer op en gingen we wat leuks doen. Ik ben erg blij dat het boekje nu af is en dat we een nieuwe fase in ons leven ingaan $\mathcal{O}$

Marcelle 


\section{Affiliation of co-authors at the time of publication}

Siavash Atashgahi ${ }^{1}$

Melike Balk

Paul L. E. Bodelier ${ }^{2}$

Ulisses N. Da Rocha ${ }^{3}$

Jan Gerritse ${ }^{4}$

Alette A. M. Langenhoff ${ }^{5}$

Marion Meima-Franke ${ }^{2}$

Charles Pijls ${ }^{6}$

Caroline Plugge ${ }^{1}$

Anja J. C. Sinke ${ }^{7}$

Hauke Smidt ${ }^{1}$

Pieter de Waard ${ }^{8}$

Bas M. van der Zaan ${ }^{4}$

${ }^{1}$ Wageningen University, Laboratory of Microbiology, Stippeneng 4, 6708 WE Wageningen, The Netherlands

${ }^{2}$ The Netherlands Institute of Ecology (NIOO-KNAW), Department of Microbial Ecology, Droevendaalsesteeg 10, 6708 PB Wageningen, The Netherlands

${ }^{3}$ VU University of Amsterdam, Department of Molecular Cell Physiology, De Boelelaan 1085, 1081 HV Amsterdam, The Netherlands

${ }^{4}$ Deltares, Subsurface and Groundwater Systems, Princetonlaan 6, 3584 CB Utrecht, The Netherlands

${ }^{5}$ Wageningen University \& Research, Department of Environmental Technology, Bornse Weilanden 9, 6708 WG, Wageningen, The Netherlands

${ }^{6}$ Tauw, Handelskade 37, 7400 AC Deventer, The Netherlands 
${ }^{7}$ BP International Limited, Sunbury on Thames, Middlesex TW167BP, UK

8 Wageningen University, BioNanoTechnology, Bornse Weilanden 9, 6700 EK Wageningen, The Netherlands 


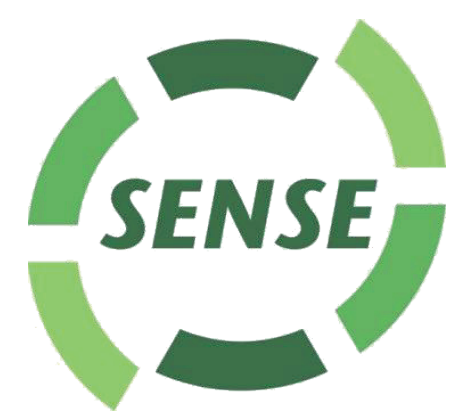

Netherlands Research School for the

Socio-Economic and Natural Sciences of the Environment

\section{I P L O M A \\ For specialised PhD training}

The Netherlands Research School for the Socio-Economic and Natural Sciences of the Environment (SENSE) declares that

Johanna Marcelle van der Waals

born on 2 January 1990 in Amersfoort, The Netherlands

has successfully fulfilled all requirements of the Educational Programme of SENSE.

Wageningen, 18 October 2018

On behalf of the SENSE board

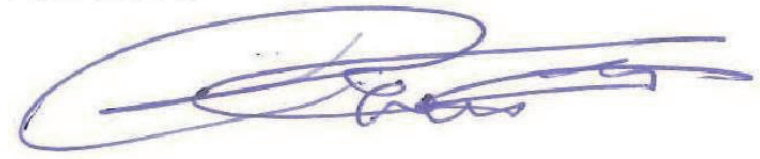

Prof. dr. Huub Rijnaarts the SENSE Director of Education

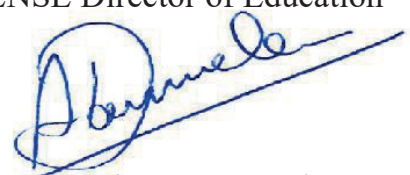

Dr. Ad van Dommelen

The SENSE Research School has been accredited by the Royal Netherlands Academy of Arts and Sciences (KNAW)

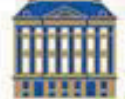

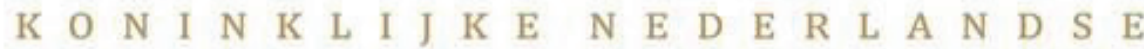

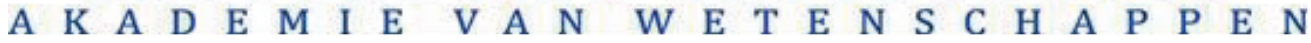




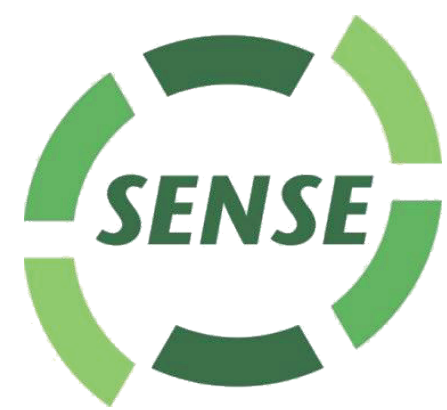

The SENSE Research School declares that Johanna Marcelle van der Waals has successfully fulfilled all requirements of the Educational $\mathrm{PhD}$ Programme of SENSE with a work load of $43.3 \mathrm{EC}$, including the following activities:

\section{SENSE PhD Courses}

o Environmental research in context (2014)

o $\quad$ Research in context activity: 'Active membership of SENSE PhD council during the period 2014-2017'

o Principles of ecological genomics (2015)

\section{Selection of other PhD and Advanced MSc Courses}

o $\quad$ Scientific writing, Wageningen Graduate Schools (2014)

o PhD presentation skills, Wageningen Graduate Schools (2014)

o ARB/SILVA workshop, Wageningen University (2014)

o Bioinformatics and metagenomics course, European Bioinformatics Institute (EMBL-EBI) (2015)

o Microbiology and biochemistry, Wageningen University (2015)

o Efficient writing strategies, Wageningen Graduate Schools (2016)

o Career perspectives, Wageningen graduate Schools (2017)

\section{Management and Didactic Skills Training}

o Assisting practicals of the MSc course 'Research Methods Microbiology' (2014-2017)

\section{$\underline{\text { Selection of Oral Presentations }}$}

o Anaerobic degradation of benzene in a biofilm reactor. ECO-BIO 2016, 7-9 March 2016, Rotterdam, The Netherlands

o $\quad$ Van de regen in de drup. Bodembreed forum, 18-19 November 2014, Lunteren, the Netherlands

o Anaerobic degradation of benzene in a biofilm reactor. CLIB-GC, 17-18 February 2016, Herdecke, Germany

o Degradation of fuel components. Engage your audience! at FEMS, 9-14 July 2017, Valencia, Spain

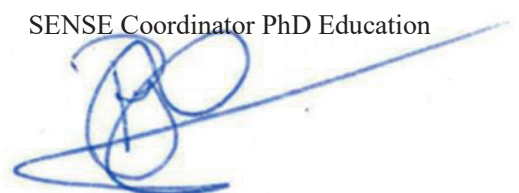

Dr. Peter Vermeulen 
The research described in this thesis was financially supported by the BE-Basic R\&D Program, which was supported through a FES subsidy from the Dutch Ministry of Economic affairs, Agriculture and Innovation (EL\&I). This project was also supported by the Dutch governmental Topsector policy.

Financial support from Wageningen University for printing this thesis is gratefully acknowledged.

Cover image adapted from: Creative Commons CCO

Printing by: Digiforce || ProefschriftMaken 



\section{Propositions}

1. Bioremediation at contaminated locations is never a one man's job.

(this thesis)

2. Gene bioaugmentation should be accepted by law, industry and general public.

(this thesis)

3. Probiotics are more effective when used in combination with adapting one's lifestyle, for example by changing the diet.

4. Van der Waals forces are essential to life on Earth.

5. Referenda are useless if they are not binding.

6. In science evaluations it is important to not only take into account the impact factor and citations, but also the relevance of the study to society.

Propositions belonging to the thesis, entitled

Degradation of fuel components

Marcelle van der Waals

Wageningen, 18 October, 2018 